\title{
DETERMINATION OF ACOUSTIC RAY \\ PATHS IN ENCLOSED SPACES
}

\section{by}

\section{Bruce Wayne Mitchell}

Thesis submitted to the Graduate Faculty of the Virginia Polytechnic Institute and State University

in partial fulfillment of the requirements for the degree of MASTER OF SCIENCE

in

Mechanical Engineering

APPROVED :

C. J. Hudst, Chairman

L. D.'Mitchell

H. H. Robertshaw

February, 1975

Blacksburg, Virginia 


\section{ACKNOWLEDGEMENTS}

The author is indebted to several people for their direct or indirect help in completing this thesis.

Special thanks go to $\mathrm{Dr}$. C. J. Hurst for the continued interest and advice he gave this author throughout this work. The author is also grateful to Drs. L. D. Mitchell and H. H. Robertshaw for their assistance in particular problem areas.

The author is indebted to the National Science Foundation and the Mechanical Engineering Department of VPI\&SU for the financial support which made this work possible.

Special thanks go to the entire family of $\mathrm{Dr}$. C. J. Hurst for their help provided "above and beyond the call of duty" to the author and his family.

The author is also thankful to the typist, , for her patience and excellent typing job. 
TABLE OF CONTENTS

PAGE

1. Introduction . . . . . . . . . . . . . . 1

2. Summary of Previous Work in Ray Acoustics . . . 4

2.1 The Eikonal Equation . . . . . . . . 6

2.2 Sound Pressure Levels Using Geometric

Acoustics . . . . . . . . . . . . 7

3. Development of Ray Tracing Equations . . . . . . 11

3.1 Preliminary Investigation . . . . . . . 11

3.2 Ray Tracing Equations . . . . . . . . . 14

3.2.1 Rooms With No S1anted Surfaces . . . . 14

3.2.2 Determination of Minimum Number of Reflections for Sound Pressure

Level Calculations . . . . . . 25

3.2.3 Ray Tracing Equations for Reflections Involving Slanted Surfaces . . . 27

4. Application of Ray Tracing Equations to Computer

Program .................. 44

4.1 Calculation of Rays . . . . . . . . . 44

4.2 Validity of Calculated Rays . . . . . . . 44

5. Sound Pressure Level Calculations . . . . . . 59

6. Application of Program . . . . . . . . . 65

6.1 Ray Tracing Plots............ 65

6.2 Comparison of Predicted and Measured Sound

Pressure Levels . . . . . . . . . 73 
TABLE OF CONTENTS - Cont'd.

\section{PAGE}

7. Conclusions . . . . . . . . . . . . 92

8. Bibliography . . . . . . . . . . . . 93

9. Appendix A . . . . . . . . . . . . . . . 94 


\section{LIST OF FIGURES}

FIGURE NO.

PAGE

1 Demonstration of Huygens Principle with Application to a Point Source ability to Image Source Technique

3 Typical Ray Path Involving A Single Reflection

4 Examples of Two Reflections From Parallel Walls (1-2-3-4) and From Perpendicular Walls $\left(1-2^{\prime}-3^{\prime}-4\right)$

5 Five General Reflection Combinations for Three Reflections

7 Enlarged View of X-Y Plane and True Size and Edge View of Slanted Barrier Shown in Figure 6

8 Reflection From A Slanted Wall To An Unslanted Wall

9 Illustration of Wall Within A Plane Concept Used In Computer Program

11 Flow Chart For Subroutine Ray Check

12 Illustration of Case Where Intersection Point Between Line And Plane Lying Outside A Wall Boundary (Wall F-G) Does Not Imply Invalid Ray 

Reflections, Randolph Classroom Reflections, Randolph Classroom And Contours of Constant Sound Pressure Level

21 Calculated Sound Pressure Levels At Positions 1-28 In Randolph Classroom And Constant Sound Pressure Level Contours

22 Comparison of Measured And Calculated dBA Sound Pressure Levels In Randolph Classroom At Positions $1,2,3,4, \ldots$ 7, Best Agreement

23 Comparison of Measured And Calculated dBA Sound Pressure Levels In Randolph Classroom 22, 23, 24, .., 28, Worst Agreement 
FIGURE NO.

PAGE

25 Comparison of Measured And Calculated

83

Octave Band Sound Pressure Levels At

Position 26 In Randolph Classroom

26 Measurement Positions In Derring Hallway

85

27 Comparison of Measured And Calculated dBA Sound Pressure Levels In Derring Hallway $1,2,3,4,5,6, \ldots, 14$

Comparison of Measured And Calculated

Overal1 Sound Pressure Levels In

Derring Hallway At Positions 1, 2, 3, $\ldots, 14$

Comparison of Measured And Calculated Octave Band Sound Pressure Levels At Position 2 In Derring Hallway, Best Agreement Octave Band Sound Pressure Levels At Position 13 In Derring Hallway, Worst Agreement 


\subsection{INTRODUCTION}

The Occupational Safety and Health Act of 1970 (OSHA $\left.^{1}\right)^{*}$ requires that workers not be exposed to noise levels greater than $90 \mathrm{dBA}$ over an eight hour period. Greater levels are permitted if the exposure time is reduced. The maximum level allowed is $115 \mathrm{dBA}$ for exposure periods of fifteen minutes or less. Engineers with little or no background in noise abatement have found themselves in the position of having to reduce the noise levels in their plants.

Therefore, the need exists for a method to predict changes in existing sound pressure levels which might result from plant modifications. Also needed is a method for predicting sound pressure levels for factories in the planning stage.

Texts by Beranek ${ }^{2}$ and others give an equation for determining total steady state sound pressure levels in "large" rooms. This equation is based on the assumption of a diffuse sound field, which can be obtained in rooms whose length, width and height do not exceed the relative proportions of $1: 1.5: 2$. For rooms which do not ronform to these ratios some error is encountered because the assumption of a diffuse sound field is violated. The magnitude of this error depends on how much the room dimensions exceed the above ratios.

Lester ${ }^{3}$, Gibbs and Jones ${ }^{4}$ and others have assumed that the acoustic energy from a source is propagated along rays emanating

* Numbers refer to entries in Bibliography. 
from the source. Because spherical spreading is assumed, the intensity at any location is assumed to be inversely proportional to the square of the distance along the ray. This approach is known as geometric acoustics. The aforementioned authors' works have shown that the geometric acoustic approach yields more accurate predictions than the room acoustics approach and has more flexibility when irregular surface areas, such as those in a factory space, are considered. Therefore, a ray tracing technique to determine the distances along sound ray paths between sources and receivers in a factory space was developed by this author. This approach was chosen because of the accuracy and flexibility in handling rooms of many shapes.

A feature of this study was the determination of the locations where each sound ray was reflected from different surfaces. Significant noise reduction at a particular receiver position might be possible if absorption material was added to those surfaces where several rays were reflected.

Knowledge of the energy propagation paths would also indicate where barriers might be erected to minimize noise at a worker location in a plant. If barriers are not feasible the source or receiver locations might be changed.

With this program the engineer has a modeling tool to check possible modifications before physically making changes in a factory. 
Before any sound pressure levels may be calculated, however, the ray paths must be determined. The emphasis in this work was determining the rays. Once a viable method for calculating ray paths was found a simplified program was devised to calculate sound pressure levels and comparisons were made with measured values in two enclosures. Reasonable agreement between the calculated and measured values indicated that it was worth proceeding with this project as a basis for further work. 


\subsection{SUMMARY OF PREVIOUS WORK IN RAY ACOUSTICS}

The use of geometric acoustics as a tool to predict sound intensity in a factory space is relatively new. Most geometric acoustics applications have been in underwater sound propagation.

The most common use of geometric acoustics is in studies investigating sound propagation where the properties of the medium vary. However, the medium in a factory space is assumed constant enough so that the ray paths are straight lines except where reflection occurs.

The basic theory of geometric acoustics centers around the assumption of spherical spreading from point sources. Figure 1(a) shows one such point source. The wave fronts which are shown are depicted as circles with increasing radil. Huyghens principle states "every point on a wave front is to be regarded as the center of a spherical distrubance spreading outwards from it, so that the resultant disturbance at a particular instant at any point in front of the advancing waves will be produced by the summation effect of all the elementary centers of disturbance."

Figure $1 b$ demonstrates the application of this principle to a point source. Wave front $A-A^{\prime}$ at time $t$ is considered to consist of an infinite number of sources from which wavelets are produced. The resultant wave front at time $(t+d t)$ is the envelope of all the wavelets at $(t+d t)$. For a slowly varying or constant speed of sound, $c$, over surface $A-A^{\prime}$ the surface $B-B^{\prime}$ will be defined approximately by 


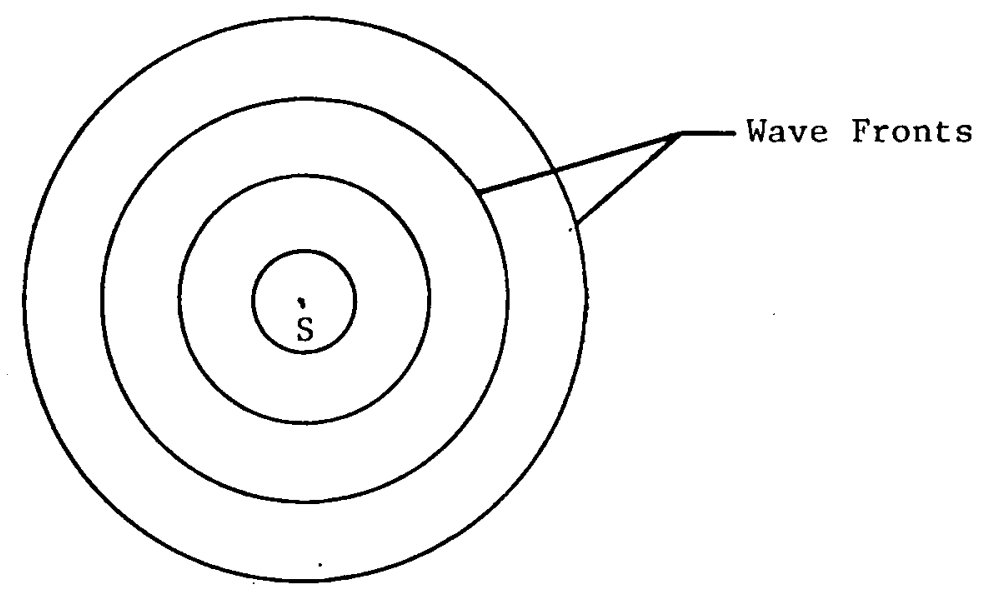

(a)

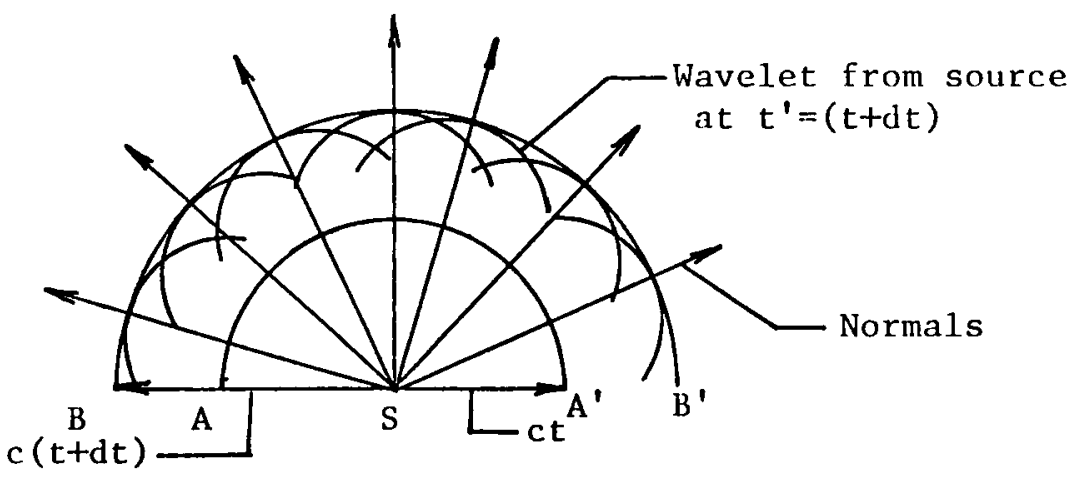

(b)

Figure 1. Demonstration of Huyghens Principle with application to a point source. 
the ends of the normals, of length cdt, to surface A-A'. A wave surface is the locus of polnts which are in the same phase of motion at a given time $t$. At the later time, $t+d t$, the wavelets define a new wave surface which is in the same phase of motion that the original wave surface was at time $t$.

\subsection{The Eikonal Equation}

officer ${ }^{5}$ and others have shown that it is possible to denote the relationship between successive wave surface positions in equation form:

$W(X+\alpha c d t, Y+\beta c d t, Z+\gamma c d t)-C_{O}\left(t_{o}+d t\right)=W(X, Y, Z)-C_{o} t_{O}$

where:

$W(X, Y, Z):$ Original wave surface

$\mathrm{C}_{\mathrm{o}}:$ A constant

$\alpha, \beta, \gamma:$ Direction cosines of the normals to original wave surface

The portion to the left of the equal sign is the new wave front and the portion to the right is the original wave front. Since $\alpha \mathrm{cdt}, \mathrm{Bcd}$, rcdt are small quantities the new wave surface can also be approximated by:

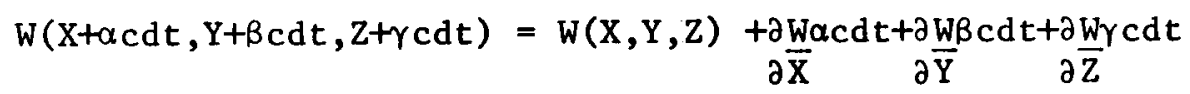

where $C=$ speed of sound

$\partial \underline{W}, \partial \underline{W}, \partial \underline{W}:$ Direction numbers for the wave surface $\partial \overline{\mathrm{X}} \partial \overline{\mathrm{Y}} \partial \overline{\mathrm{Z}}$

If equations (1) and (2) are equated:

$$
\alpha \frac{\partial W}{\partial X}+\beta \frac{\partial W}{\partial Y}+\gamma \frac{\partial W}{\partial Z}=\frac{C_{O}}{C}
$$


Since the direction numbers are proportional to the direction cosines, and the direction cosines $\alpha, \beta, \gamma$ can be expressed in terms of derivatives along the normals, several algebraic munipulations yield:

$$
\left(\frac{\partial W}{\partial X}\right)^{2}+\left(\frac{\partial W}{\partial Y}\right)^{2}+\left(\frac{\partial W}{\partial Z}\right)^{2}=1
$$

Which is known as the Eikonal equation, the fundamental equation of ray acoustics. A solution of this equation will be a good approximation to the wave equation.

\subsection{Sound Pressure Levels Using Geometric Acoustics}

Previous work done in sound pressure level predictions has employed a technique known as the image source method. This method consists of considering an infinite array of imaginary sources to exist, each radiating to the receiver under study. Each imaginary source yields a propagation path equivalent to one of the multiple reflection ray paths existing within the actual room.

Bolt, Doak and Westervelt ${ }^{6}$ used the image technique to study reverberation time and mean free path. In their study they contended that many sounds of speech and music more nearly resemble pulsed wave trains than the abruptly terminated continuous sounds commonly used in reverberation time measurement. They examined analytically the response of a room to a short pulse. The walls were replaced by an array of pulsed image sources which were then considered statistically. Experiments in a hard-walled rectangular room were used to compare with calculated values. Several discrete reflections were measured and 
correlated with calculations. The pulses merged into a more or less continuous background sound pressure level after a time. This level was calculated and confirmed experimentally.

Krokstad, Strom and Sorsdal ${ }^{7}$ studied the space and time distribution of reflections in concert halls. Their calculation procedure used a mathematical model of a hall which was excited by a sound pulse emitted from a fixed point source. Energy was represented by rays equally distributed over the whole or over a selected part of a solid angle. The life history of each ray was calculated, assuming geometrical reflection at all surfaces, until the ray struck the audience area where it was assumed the ray was totally absorbed. The points of reflection for a11 emitted rays and the time delay of impingement relative to the direct sound were calculated. The direction of the rays was computed from equations derived by stenseng ${ }^{8}$.

Stenseng assumed a uniform distribution of rays emanating from the center of a spherical source. With the location of each ray known its equation in polar coordinates was derived. Reflection points were calculated by combining the ray's equation and the equation for a surface. Each ray was repeatedly reflected until it was absorbed or became longer than a given maximum. In their discussion they stated that some limitations existed with this approach. The most severe of these was the limited frequency range over which results were valid. This was because in ray acoustics the wave lengths corresponding to the lowest frequency of the sound need to be small compared to the 
linear dimensions of the room and its surfaces. For large rooms accuracy may be expected to be good for those frequencies above 100 hertz.

The image technique was used by Gibbs and Jones ${ }^{4}$ to predict variations in sound pressure levels in a rectangular room possessing various absorption configurations. Patches of acoustic absorption material were centrally located on each wall. Absorption values in the calculations were then chosen according to. the location of the reflection points. Experiments were run in a rectangular room with a single patch and with six patches. The results were compared with predicted sound pressure levels using both the classical room acoustics and image techniques. Results showed that the image technique more closely approximated the experimental results than did predictions using the classical expression. The predictions from the image approach gave significantly better agreement with measured results when the absorption was distributed between all six surfaces of the room. In the conclusions it was noted that this improved agreement was gained at the expense of computing time.

An important aspect, for this investigation, of the work of Gibbs and Jones was the investigation of the effect of the angle of incidence on the absorption coefficient. They determined the sound pressure level distribution assuming a random incidence absorption coefficient, and also assuming that the absorption coefficient varied with the angle of incidence. The difference between the two distributions was said to be too slight to justify the additional computing time. But they also 
noted that it was difficult to assume that the absorption is a function of the angle of incidence because of the lack of reliable information as to the nature of the angular variation of the absorption coefficient.

Lester ${ }^{3}$ used the image technique to predict sound pressure levels in rectangular and "L-shaped" rooms. Comparisons were made with the experimental works of Lowe ${ }^{9}$, Beranek ${ }^{2}$ and with measurements made in a scale model of a large, low-ceilinged room. The results of Lester's predictions showed good agreement with the measured sound levels. Initially Lester considered using the absorption as a function of incidence angle but all of his results were computed using a random absorption coefficient.

Barron ${ }^{10}$ used the image method to derive expressions to predict the average number of reflections occurring after the emission of a signal from a source. The decay with time of the reverberant field strength was also computed as a function of the distance between source and receiver. His image model predicted a non-exponential decay of the case of a highly absorbent floor. If highly reflective surfaces existed in a room, the model predicted no more than the generally assumed exponential decay as the distance between the source and receiver increased. 


\subsection{DEVELOPMENT OF RAY TRACING EQUATIONS}

\subsection{Preliminary Investigation}

Several methods were considered for calculating the reflection points of the sound rays on the surfaces within a room. The image method, along the lines of Lester's ${ }^{2}$ thesis, was studied. This was an acceptable method if the walls of the room were like those shown in Figure 2a. However, the image technique does not easily handle slanted surfaces as illustrated in Figure $2 \mathrm{~b}$. Computer time was somewhat lengthy in this approach which placed a second limitation on its use.

Use of the direction cosines of the ray paths was considered next. Figure 3 shows a typical ray path involving a single reflection. Snells law tells us that the angle of incidence of a sound ray equals its angle of reflection. Expressions may be written for the direction cosines of the incident and reflected rays. By manipulating these expressions the reflection point may be determined. This method proved cumbersome, and unique solutions for the path from a given source to a given receiver were indeterminant when more than one reflection from a room boundary was involved. Iteration was considered, but the computer time would have been excessive in comparison with equations which could be used to solve for the reflection points directly.

The method decided upon was based on the use of equations which could solve for the reflection points directly. Different equations were used depending upon whether the reflection locations were to be determined for one, two, or " $N$ " number of reflections. Because no 


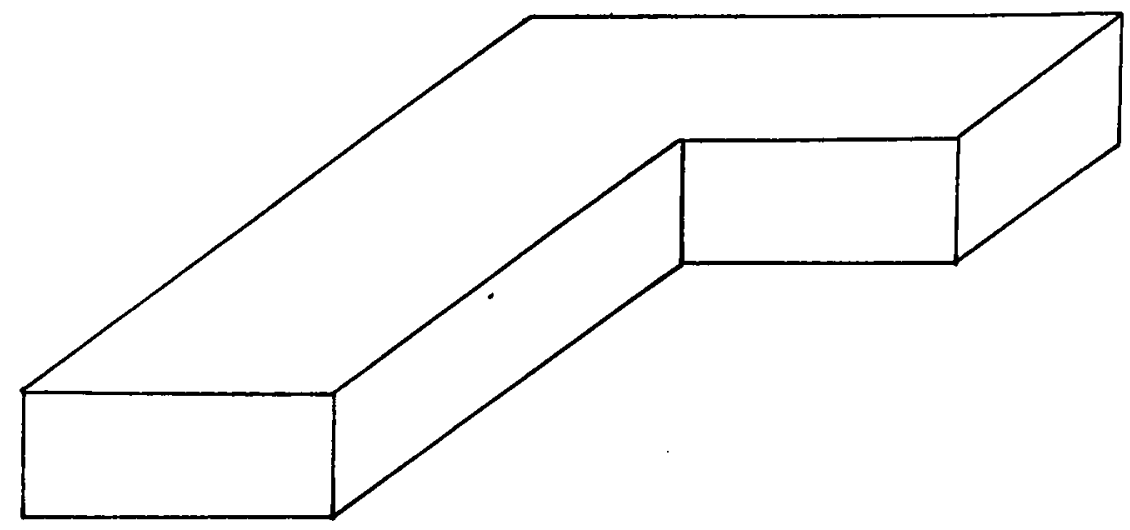

(a) Room Acceptable to Image Technique.

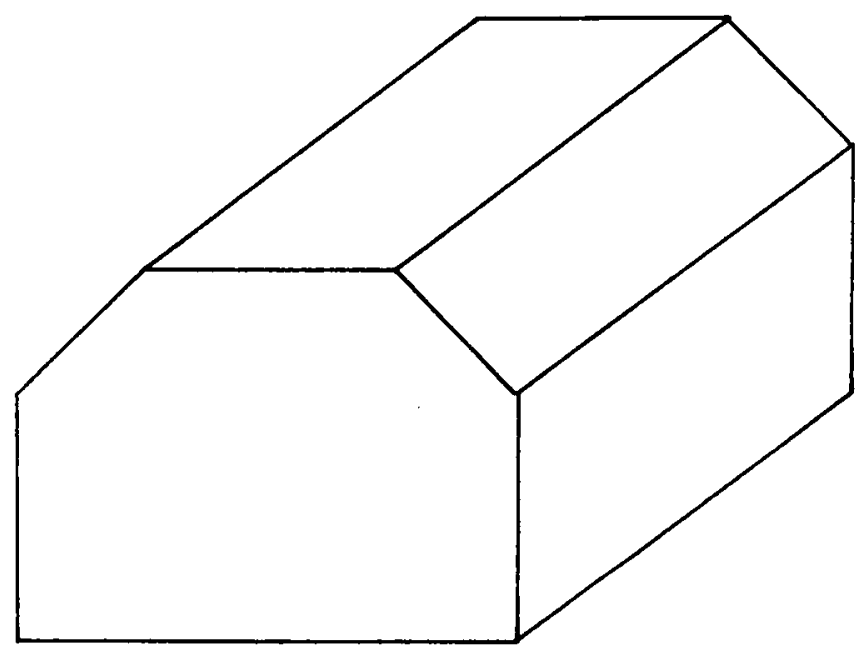

(b) Room not easily handled by image technique.

Figure 2. Room Configurations and their Acceptability to Image Source Technique. 


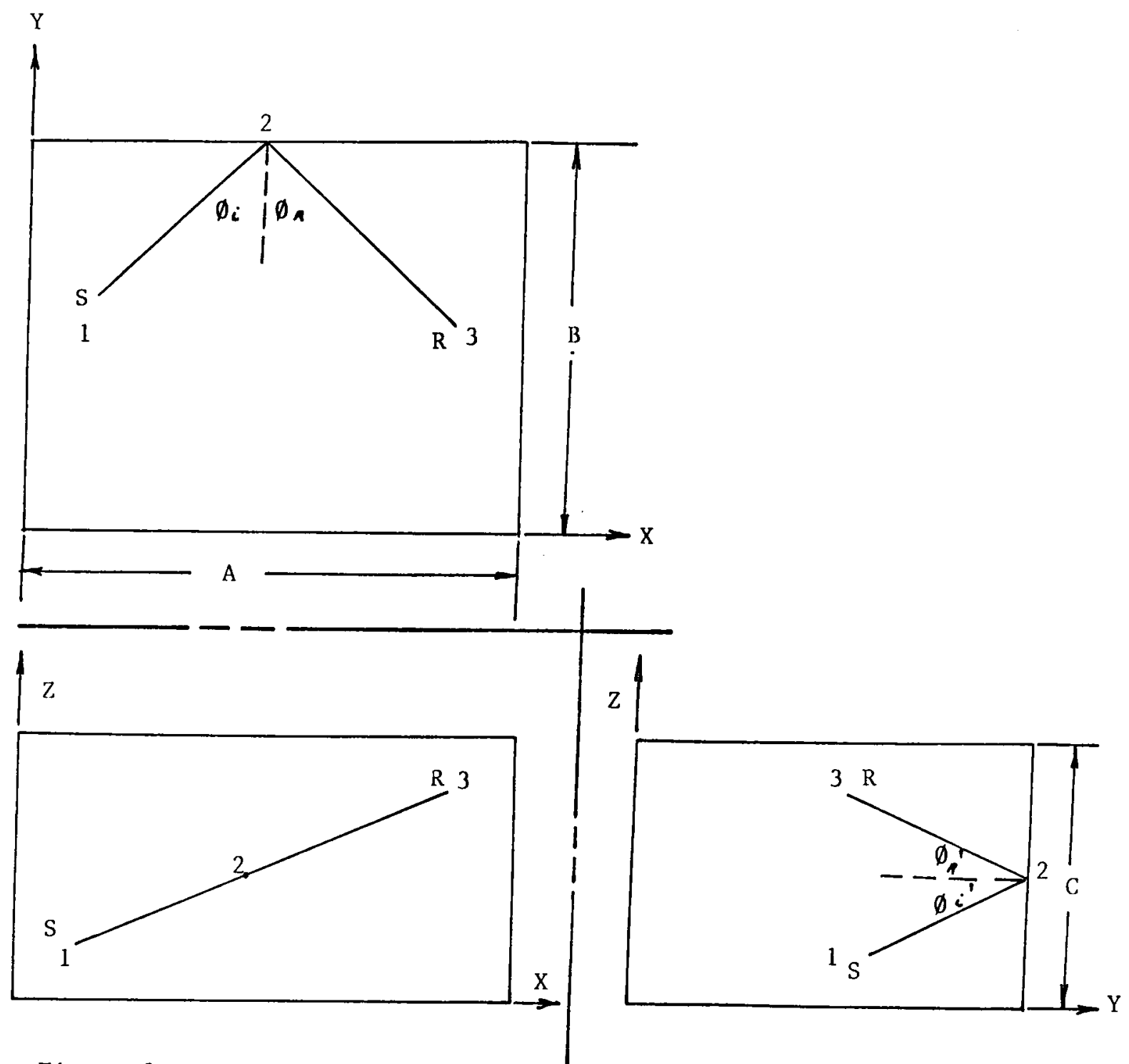

Figure 3. Typical ray path involving a single reflection. 
iteration was necessary computer time was reduced. The next section discusses the development of these ray tracing equations and subsequent application of these equations to a computer program.

\subsection{Ray Tracing Equations}

The final form of the ray tracing equations was determined from trigonometric and geometric considerations. Snell's law was used to determine the coordinates of all reflection points. This procedure was applied, for example, to. surfaces like those shown in Figures $2 a$ and $2 b$. The surfaces in Figure $2 a$ will be discussed first.

\subsubsection{Rooms with No Slanted Surfaces}

Consider the three views of the room shown in Figure 3. A sound ray is shown undergoing one reflection from the back wall $\left(Y_{2}=B\right)$. A smooth surface is assumed.

One knows from Snell's law that the angle of incidence $\left(\emptyset_{i}\right)$ equals the angle of reflection $\left(\phi_{r}\right)$.

$$
\emptyset_{i}=\emptyset_{r}
$$

When a reflection occurs the incident and reflected sound rays define a plane which must be perpendicular to the wall at which the reflection occurs. With this information the coordinates of the reflection point can be determined.

For the ray shown in Figure 3 the reflection occurs on the wall which lies in the plane $Y=B$. Since the reflection point is on the wall, it is evident that the $\mathrm{Y}$ coordinate is also equal to $\mathrm{B}$. It is only necessary to solve for the $X$ and $Z$ coordinates of the reflection point. 
The solution proceeds as follows:

Snel1's law (Equation 5) may be re-written:

$$
\begin{aligned}
& \operatorname{Tan}\left(\emptyset_{i}\right)=-\operatorname{Tan}\left(\emptyset_{r}\right) \\
& \frac{\mathrm{Y}_{2}-\mathrm{Y}_{1}}{\mathrm{X}_{2}-\mathrm{X}_{1}}=\frac{-\left(\mathrm{Y}_{3}-\mathrm{Y}_{2}\right)}{\mathrm{X}_{3}-\mathrm{X}_{2}}
\end{aligned}
$$

There is only one unknown, $\mathrm{x}_{2}$, in Equation (5b). This can be solved for:

$$
X_{2}=\frac{X_{1}\left(Y_{2}-Y_{3}\right)+X_{3}\left(Y_{2}-Y_{1}\right)}{2 Y_{2}-Y_{1}-Y_{3}}
$$

The remaining unknown, $z_{2}$, may be determined in one of two ways. Since $\mathrm{X}_{2}$ is known its value may be used to calculate $z_{2}$. The reflection, when viewed in the $X-Z$ plane in Figure 3 , is a straight line because the source, receiver and reflection point all lie in the same plane. Therefore, the slope of ray segment $1-2$ is the same as the entire ray $1-3$, i.e.:

$$
\frac{z_{3}-z_{1}}{x_{3}-x_{1}}=\frac{z_{2}-z_{1}}{x_{2}-x_{1}}
$$

Which, when solved for $z_{2}$ yields:

$$
z_{2}=\frac{z_{1}\left(x_{3}-x_{2}\right)+z_{3}\left(x_{2}-x_{1}\right)}{x_{3}-x_{1}}
$$

If the source and receiver are both the same distance from the $Y-Z$ plane then $X_{3}$ equals $X_{1}$ and Equation (8) becomes indeterminant. To avoid this possibility Snells law may be applied to the view of 
the ray path in the $\mathrm{Y}-\mathrm{Z}$ plane:

$$
\frac{Z_{3}-Z_{2}}{Y_{3}-Y_{2}}=\frac{-\left(Z_{2}-Z_{1}\right)}{Y_{2}-Y_{1}}
$$

This Equation, when rearranged may be solved for $\mathrm{z}_{2}$ :

$$
Z_{2}=\frac{Z_{1}\left(Y_{3}-Y_{2}\right)+Z_{3}\left(Y_{1}-Y_{2}\right)}{Y_{1}+Y_{3}-2 Y_{2}}
$$

The denominator in this equation cannot be zero because that would mean that the source and receiver lay in, the same plane as the wall from which the reflection occurs. (i.e., $\mathrm{Y}_{1}=\mathrm{Y}_{3}=\mathrm{Y}_{2}=\mathrm{B}$ ).

In this manner all three coordinates of the reflection point have been determined from equations (6) and (10) and the known plane of reflection.

This procedure may be repeated for one reflection for each of the remaining walls shown in Figure 3 .

Two possible reflection combinations may occur when a sound ray undergoes two reflections. Ray path 1-2-3-4 in Figure 4 is an example of reflections from parallel walls. Ray path $1-2^{\prime}-3^{\prime}-4$ in Figure 4 is an example of reflections from perpendicular walls.

The case of reflections from parallel walls will be considered first. The known coordinates for the reflection points 2 and 3 of the ray path 1-2-3-4 are $Y_{2}=B$ and $Y_{3}=0$. Snell's law may be applied to the sound ray path in the $X-Y$ and $Y-Z$ planes to obtain the necessary equations to derive the unique reflection points 2 and 3 . Since the 

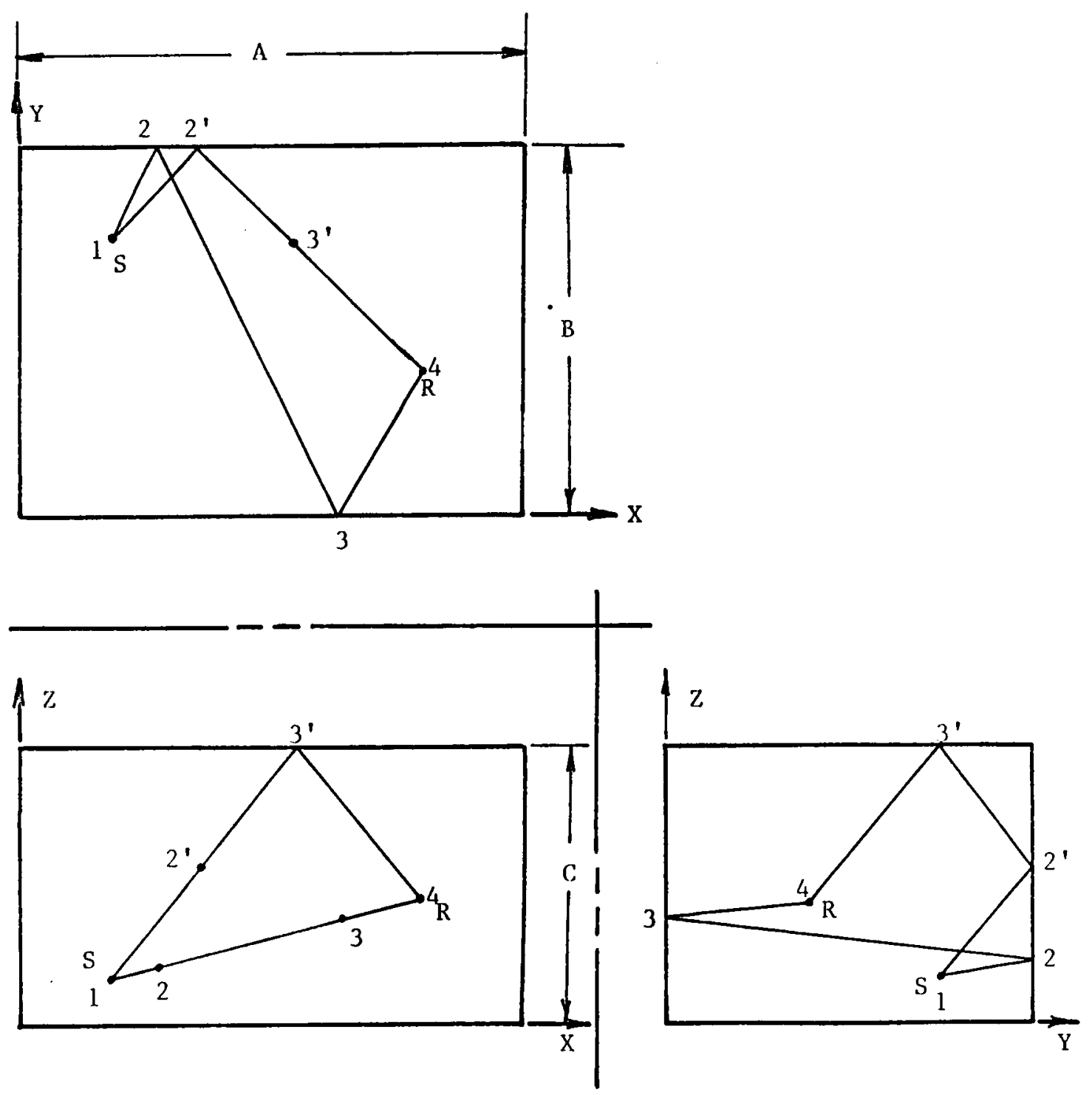

Figure 4. Examples of two reflections from parallel walls (1-2-3-4) and from perpendicular walls $\left(1-2^{\prime}-3^{\prime}-4\right)$. 
reflections occur on parallel surfaces the view in the $X-Z$ plane shows all points lying in the same plane. This fact is used to obtain the equations to determine points 1 and 2 . These equations may be rearranged to determine the necessary values, although not as simply as in the case of one reflection. For example, in the $X-Y$ plane we can show that:

$$
\frac{Y_{1}-Y_{2}}{X_{1}-X_{2}}=\frac{Y_{3}-Y_{4}}{X_{3}-X_{4}}
$$

In that same view we can also see that

$$
\frac{Y_{2}-Y_{3}}{X_{2}-X_{3}}=\frac{-\left(Y_{1}-Y_{2}\right)}{X_{1}-X_{2}}
$$

Equations (10) and (11) may be rearranged to eliminate one of the two unknowns, $x_{3}$. The resulting equation when solved for $x_{2}$ is:

$$
X_{2}=\frac{X_{1}\left(2 Y_{3}-Y_{4}-Y_{2}\right)+X_{4}\left(Y_{1}-Y_{2}\right)}{2 Y_{3}-2 Y_{2}+Y_{1}-Y_{4}}
$$

Now that $\mathrm{X}_{2}$ has been determined $\mathrm{x}_{3}$ may be calculated from Equation (11)

$$
X_{3}=\frac{X_{1}\left(Y_{2}-Y_{3}\right)+X_{2}\left(Y_{3}-2 Y_{2}+Y_{1}\right)}{Y_{1}-Y_{2}}
$$

The coordinates $z_{2}$ and $z_{3}$ may be determined by the application of Snells in the $Y-Z$ plane in a similar manner. These equations are:

$$
\begin{aligned}
& Z_{2}=\frac{Z_{1}\left(2 Y_{3}-Y_{4}-Y_{2}\right)+Z_{4}\left(Y_{1}-Y_{2}\right)}{2\left(Y_{3}-Y_{2}\right)+Y_{1}-Y_{4}} \\
& Z_{3}=\frac{Z_{2}\left(Y_{3}-Y_{4}\right)+Z_{4}\left(Y_{3}-Y_{2}\right)}{2 Y_{3}-Y_{2}-Y_{4}}
\end{aligned}
$$


To use these equations for walls parallel in the $\mathrm{X}-\mathrm{Y}$ or $\mathrm{Y}-\mathrm{Z}$ coordinate planes it is only necessary to interchange the coordinates. For example, suppose the two parallel walls in question were perpendicular to the X-axis. The previously derived equations $(13,14,15,16)$ may be used by interchanging each $X$ for $a \mathrm{Y}$ and vice versa. This produces the same result as rotating the X-Y plane $90^{\circ}$ so that the $X$ and $Y$ coordinates exchange locations.

The situation for the case of successive reflections from perpendicular walls is more complicated. A typical ray path of this kind is ray path 1-2'-3'-4 shown in Figure 4. The known coordinates (in addition to the source and receiver) are $\mathrm{Y}_{2}{ }^{\prime}=\mathrm{B}$ and $\mathrm{Z}_{3}{ }^{\prime}=\mathrm{C}$. Once again equations can be derived by applying the two aforementioned criteria - Snell's law and the fact that any three consecutive points define a plane. It is important to note that the source, the receiver, and the two intermediate reflection points do not lie in the same plane, as was the case for parallel walls. Only any three successive points lay in the same plane, e.g., points $1-2^{\prime}-3^{\prime}$ and $2^{\prime}-3^{\prime}-4$; for the ray in question.

The general form of the ray tracing equations for two reflections involving perpendicular walls can be found by the procedure indicated above, to be:

$$
\begin{aligned}
& X_{2}^{\prime}=\frac{X_{1}\left(Y_{2}^{\prime}-Y_{4}\right)+X_{4}\left(Y_{2}^{\prime}-Y_{1}\right)}{2 Y_{2}-Y_{1}-Y_{4}} \\
& X_{3}^{\prime}=\frac{X_{1}\left(Z_{3}^{\prime}-Z_{4}\right)+X_{4}\left(Z_{3}^{\prime}-Z_{1}\right)}{2 Z_{3}^{\prime}-Z_{1}-Z_{4}}
\end{aligned}
$$




$$
\begin{aligned}
& Z_{2}^{\prime}=\frac{Z_{1}\left(Y_{4}-Y_{2}\right)+Z_{3}^{\prime}\left(2 Y_{1}-2 Y_{2}\right)+Z_{4}\left(Y_{2}^{\prime}-Y_{1}\right)}{Y_{1}+Y_{4}-2 Y_{2}^{\prime}} \\
& Y_{3}^{\prime}=\frac{Y_{1}\left(Z_{4}-Z_{3}\right)-Y_{2}^{\prime}\left(Z_{4}-Z_{3}\right)+Y_{4}\left(Z_{2}^{\prime}-Z_{1}\right)}{Z_{2}^{\prime}-Z_{1}}
\end{aligned}
$$

These equations apply to successive reflections from a wall lying in the plane $\mathrm{Y}=$ constant and a wall lying in the plane $\mathrm{Z}=$ constant. For other combinations involving perpendicular walls the equations are of the same form as equations 16-19, but, as in the case of parallel walls, the $X, Y$ and $Z$ values are interchanged to solve for all possible two reflection combinations.

While there are only two types of reflection combinations when two reflections occur, the situation changes when three successive reflections are considered. There are five general reflection combinations. These are shown in Figure 5. Figure 5a consists of two successive reflections from parallel walls and a third reflection from a wall perpendicular to the two parallel walls. Figure 5b is a reflection combination involving the reverse of Figure 5a, i.e., the first two successive reflections (1-2-3) are from perpendicular wa11s and the last two successive reflections (3-4-5) are from parallel walls. Figure $5 c$ shows three successive reflections from parallel wa11s.

As in the case of two successive reflections, one coordinate location of each of the three reflection points are known. As an example, the known coordinates in Figure 5a are: 

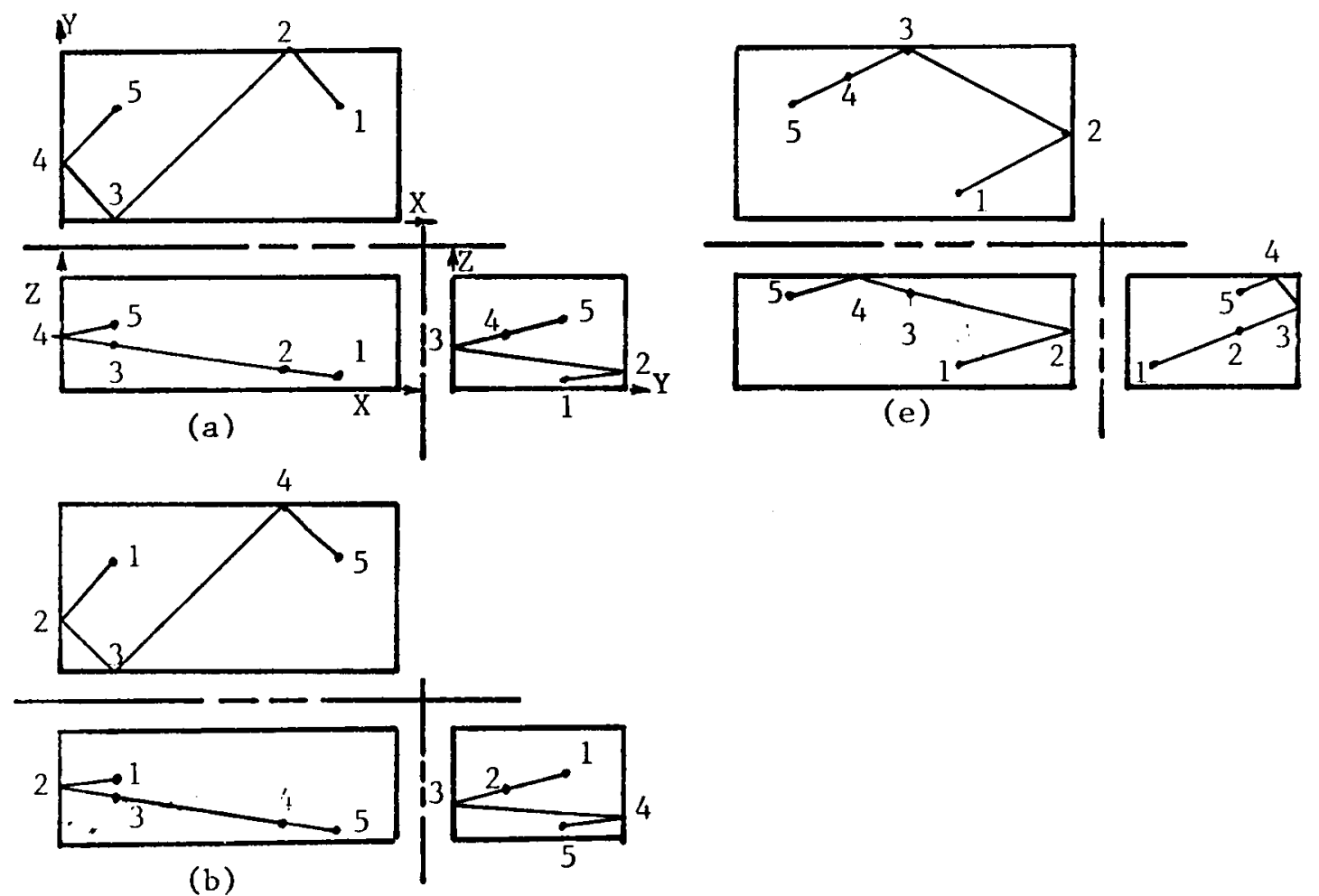

Maximum $X$ Dimension $=\Lambda$

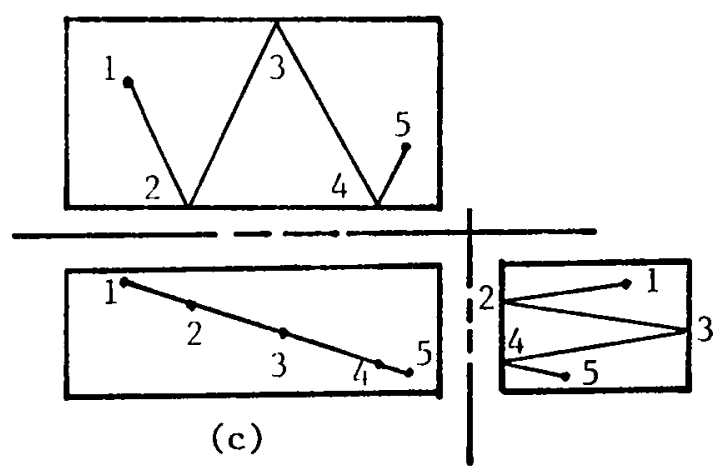

Maximum $Y$ Dimension $=B$

Maximum Z Dimension $=\mathrm{C}$

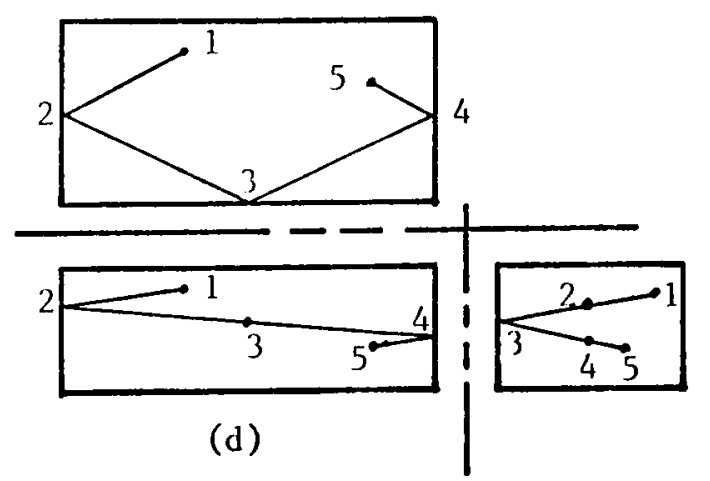

Figure 5. Five genera1 reflection combinations for three reflections. 


$$
\begin{aligned}
& Y_{2}=B \text { For Point } 2 \\
& Y_{3}=0 \text {. For Point } 3 \\
& X_{4}=0 \text {. For Point } 4
\end{aligned}
$$

With this information the ray tracing equations for three successive reflections can be derived. The procedure parallels that for the single and double reflection cases. The complexity of the algebra is increased since there are three reflection points to determine, but the method of determining the necessary equations is the same. The genera1 ray tracing equations for three successive reflections are found to be:

Figure 5a: (Two X-Z planes, followed by a $\mathrm{Y}-\mathrm{Z}$ plane)

$$
\begin{aligned}
& Z_{4}=\frac{Z_{5}\left(X_{1}-X_{4}\right)+Z_{1}\left(X_{5}-X_{4}\right)}{X_{1}+X_{5}-2 X_{4}} \\
& Z_{2}=\frac{Z_{5}\left(Y_{2}-Y_{1}\right)+Z_{1}\left(Y_{5}+Y_{2}-2 Y_{3}\right)}{2 Y_{2}-2 Y_{3}-Y_{1}+Y_{5}} \\
& Z_{3}=\frac{Z_{1}\left(Y_{2}-Y_{3}\right)-Z_{2}\left(2 Y_{2}-Y_{1}-Y_{3}\right)}{Y_{1}-Y_{2}} \\
& X_{2}=\frac{X_{1}\left(Y_{5}-2 Y_{3}+Y_{2}\right)+2 X_{4}\left(Y_{2}-Y_{1}\right)+X_{5}\left(Y_{1}-Y_{2}\right)}{\left(2 Y_{2}-2 Y_{3}-Y_{1}+Y_{5}\right)} \\
& X_{4}=\frac{Y_{1}\left(X_{4}-X_{5}\right)+Y_{2}\left(X_{5}-X_{4}\right)+Y_{5}\left(X_{2}-X_{1}\right)}{\left(X_{2}-X_{1}\right)} \\
& X_{3}=\frac{X_{1}\left(Y_{3}-Y_{2}\right)+X_{2}\left(2 Y_{2}-Y_{3}-Y_{1}\right)}{\left(Y_{2}-Y_{1}\right)}
\end{aligned}
$$


Figure 5b: (X-Z plane followed by two $X-Y$ planes)

$$
\begin{aligned}
& Z_{2}=\frac{Z_{1}\left(X_{2}-X_{5}\right)+Z_{5}\left(X_{2}-X_{1}\right)}{2 X_{2}-X_{1}-X_{5}} \\
& Z_{3}=\frac{Z_{1}\left(2 Y_{4}-Y_{3}-Y_{5}\right)-Z_{5}\left(Y_{3}-Y_{1}\right)}{2 Y_{4}-2 Y_{3}-Y_{5}+Y_{1}} \\
& Z_{4}=\frac{Z_{1}\left(Y_{4}-Y_{3}\right)-Z_{3}\left(Y_{4}-2 Y_{3}+Y_{1}\right)}{Y_{3}-Y_{1}} \\
& X_{4}=\frac{X_{2}\left(2 Y_{4}-2 Y_{5}\right)+X_{5}\left(Y_{1}+Y_{4}-2 Y_{3}+X_{1}\left(Y_{5}-Y_{4}\right)\right.}{Y_{1}-2 Y_{3}+2 Y_{4}} \\
& X_{3}=\frac{X_{4}\left(2 Y_{4}-Y_{3}-Y_{5}\right)+X_{5}\left(Y_{3}-Y_{4}\right)}{Y_{4}-Y_{5}} \\
& Y_{2}=\frac{Y_{3}\left(X_{1}-X_{2}\right)-Y_{4}\left(X_{1}-X_{2}\right)+Y_{1}\left(X_{4}-X_{3}\right)}{X_{4}-X_{3}}
\end{aligned}
$$

Figure 5c: (Reflection from two parallel $\mathrm{Y}-\mathrm{Z}$ planes)

$$
\begin{aligned}
& Z_{2}=\frac{Z_{1}\left(2 Y_{3}-2 Y_{4}+Y_{5}-Y_{2}\right)-Z_{5}\left(Y_{2}-Y_{1}\right)}{2 Y_{3}-2 Y_{2}-2 Y_{4}+Y_{5}+Y_{1}} \\
& Z_{4}=\frac{Z_{5}\left(Y_{2}-Y_{1}\right)+Z_{2}\left(Y_{5}-Y_{4}\right)-Z_{1}\left(Y_{5}-Y_{4}\right)}{\left(Y_{2}-Y_{1}\right)} \\
& X_{4}=\frac{X_{5}\left(2 Y_{3}-2 Y_{2}-Y_{4}+Y_{1}\right)+X_{1}\left(Y_{5}-Y_{4}\right)}{Y_{5}-2 Y_{4}+2 Y_{3}-2 Y_{2}+Y_{1}} \\
& X_{2}=\frac{X_{1}\left(Y_{5}-Y_{4}\right)+X_{5}\left(Y_{1}-Y_{2}\right)+X_{4}\left(Y_{2}-Y_{1}\right)}{Y_{5}-Y_{4}} \\
& X_{3}=\frac{X_{4}\left(Y_{1}-Y_{2}\right)+X_{2}\left(Y_{4}-Y_{3}\right)+X_{1}\left(Y_{3}-Y_{4}\right)}{Y_{1}-Y_{2}}
\end{aligned}
$$




$$
Z_{3}=\frac{Z_{4}\left(Y_{2}-Y_{1}\right)+Z_{2}\left(Y_{3}-Y_{4}\right)-Z_{1}\left(Y_{3}-Y_{4}\right)}{Y_{2}-Y_{1}}
$$

Figure 5d: (Reflection from $Y-Z, X-Z, Y-Z$ planes)

$$
\begin{aligned}
& Z_{3}=\frac{Z_{5}\left(Y_{1}-Y_{3}\right)+Z_{1}\left(Y_{5}-Y_{3}\right)}{Y_{5}-2 Y_{3}+Y_{1}} \\
& z_{2}=\frac{z_{1}\left(2 x_{4}-x_{2}-x_{5}\right)+z_{5}\left(x_{1}-x_{2}\right)}{2 x_{4}-2 x_{2}-x_{5}+x_{1}} \\
& z_{4}=\frac{z_{1}\left(x_{4}-x_{2}\right)-z_{2}\left(x_{4}+x_{1}-2 x_{2}\right)}{x_{2}-x} \\
& x_{4}=\frac{Y_{3}\left(2 x_{5}-2 x_{4}\right)+y_{1}\left(x_{4}-x_{5}\right)+y_{5}\left(2 x_{2}-x_{1}-x_{4}\right)}{2 x_{2}-2 x_{4}+x_{5}-x_{1}} \\
& X_{3}=\frac{X_{5}\left(Y_{3}-Y_{4}\right)+X_{4}\left(2 Y_{4}-Y_{3}-Y_{5}\right)}{Y_{4}-Y_{5}} \\
& \mathrm{Y}_{2}=\frac{\mathrm{Y}_{5}\left(\mathrm{X}_{1}-\mathrm{x}_{2}\right)+\mathrm{Y}_{4}\left(\mathrm{x}_{2}-\mathrm{x}_{1}\right)+\mathrm{Y}_{1}\left(\mathrm{x}_{5}-\mathrm{x}_{4}\right)}{\mathrm{X}_{5}-\mathrm{x}_{4}}
\end{aligned}
$$

Figure 5e: (Reflection from $Y-Z, X-Z, X-Y$ planes)

$$
\begin{aligned}
& Y_{4}=\frac{Y_{1}\left(Z_{5}-Z_{4}\right)+\left(2 Z_{4}-2 Z_{5}\right)+Y_{5}\left(Z_{4}-Z_{1}\right)}{2 Z_{4}-Z_{5}-Z_{1}} \\
& Z_{3}=\frac{Z_{5}\left(Y_{1}-Y_{3}\right)-Z_{4}\left(Y_{1}-Y_{3}\right)+Z_{1}\left(Y_{4}-Y_{5}\right)}{\left(Y_{4}-Y_{5}\right)} \\
& Y_{2}=\frac{Y_{3}\left(2 X_{1}-2 X_{2}\right)+Y_{1}\left(X_{5}-X_{2}\right)+Y_{5}\left(X_{2}-X_{1}\right)}{X_{1}+X_{5}-2 X_{2}} \\
& X_{3}=\frac{X_{1}\left(Y_{3}-Y_{5}\right)-X_{2}\left(Y_{3}-Y_{5}\right)+X_{5}\left(Y_{1}-Y_{2}\right)}{\left(Y_{1}-Y_{2}\right)}
\end{aligned}
$$




$$
\begin{aligned}
& X_{4}=\frac{X_{1}\left(Y_{4}-Y_{5}\right)-X_{2}\left(Y_{4}-Y_{5}\right)+X_{5}\left(Y_{1}-Y_{2}\right)}{\left(Y_{1}-Y_{2}\right)} \\
& Z_{2}=\frac{Z_{5}\left(X_{1}-X_{2}\right)-Z_{4}\left(X_{1}-X_{2}\right)-Z_{1}\left(X_{5}-X_{4}\right)}{\left(X_{4}-X_{5}\right)}
\end{aligned}
$$

The five ray tracing equation sets derived can be used to determine all of the possible three reflection combinations. For example, in Figure 5a reflections are shown occurring on the $\mathrm{Y}_{2}=\mathrm{B}$ wall, $\mathrm{Y}_{3}=$ 0 . wall and $x_{4}=0$ wall. To use the derived equations, equations (21)(26), for a reflection from the $x_{2}=0 ., x_{3}=A$ and $Y_{4}=0$. walls, the $Y$ values are substituted in the equation for the $X$ values and vice versa. By switching the $X, Y$ and $Z^{\prime} s$ in the general equations a11 the possible combinations are calculated.

\subsubsection{Determination of Minimum Number of Reflections}

for Sound Pressure Level Calculations

The same procedure can be applied to four or more reflections. Ray tracing equations for four reflections would require equations to solve for eight unknown coordinates. The additional reflection would also add additional reflection sequence combinations; each with four unknown coordinate pairs. In view of the increased complexity of solving all of the necessary ray tracing equations for four or more reflections calculations were limited to those sound rays undergoing three or fewer reflections.

Although four or more reflections would require much additional analysis, the decision to consider only three reflections was not 
made for this reason only. Once the ray paths were determined they were to be used to calculate sound pressure levels. In section 1.0 sound intensity was said to be inversely proportional to the ray path length squared:

$$
I_{\text {Sound }} \propto \frac{1}{R^{2}}
$$

where:

$$
\begin{aligned}
& I_{\text {Sound }}=\text { Sound Intensity } \\
& \mathrm{R}=\text { Distance along ray path }
\end{aligned}
$$

Unless the room in question is extremely narrow in one dimension most three reflection paths will be longer than the two reflection paths. Paths involving four reflections would generally be longer than those involving three reflections. Therefore, it can be seen from equation (51) that the acoustic energy arriving along ray paths involving more than three reflections would be appreciably lower, in general than that arriving along a shorter path.

Another factor in determining the necessary number of reflections is the absorption property of the walls. When a sound ray strikes a surface its intensity is reduced proportional to the absorptive characteristics of the surface. Most surfaces will absorb a portion of the intensity of the impinging ray so the intensity also decreases as the number of reflections increase.

Finally, it is worth noting that Lester ${ }^{3}$ used a maximum of three reflections to predict sound pressure levels. The good agreement of his results with those he measured lends credence to this procedure. 
3.2.3 Ray Tracing Equations for Reflections Involving Slanted Surfaces

A rectangular room is shown in Figure 6 that contains a "slanted" surface, i.e., one that is perpendicular to only one of the coordinate planes, in this example, the X-Y plane. Slanted surfaces can occur in factory spaces as barriers and slanted walls so it was necessary to derive the necessary ray tracing equations for this application.

In the previous section the ray tracing equations were developed with the reflecting surfaces perpendicular to one of the coordinate axes. To use the same type of analysis for slanted surfaces it was necessary to use a rotated set of coordinate axes like the primed coordinates shown in Figure 6 . By rotating a set of axes until the $X^{\prime}$ axis was parallel to the reflecting surface in question the necessary ray tracing equations could be developed.

The first step in this analysis was to determine a relationship between the initial coordinate axes and the rotated axes. Figure 7 shows an enlarged view of the $X-Y$ plane and the true size and edge views of the slanted plane in Figure 6 . Two axes $\left(X^{\prime}-Y^{\prime}\right)$ were constructed parallel and perpendicular to the slanted surface with the source as the zero location of the primed axes. The angle is the angle formed by the $X$ and $X^{\prime}$ coordinate axes. Since point 2 must lie on the slanted plane the following expressions were derived: 


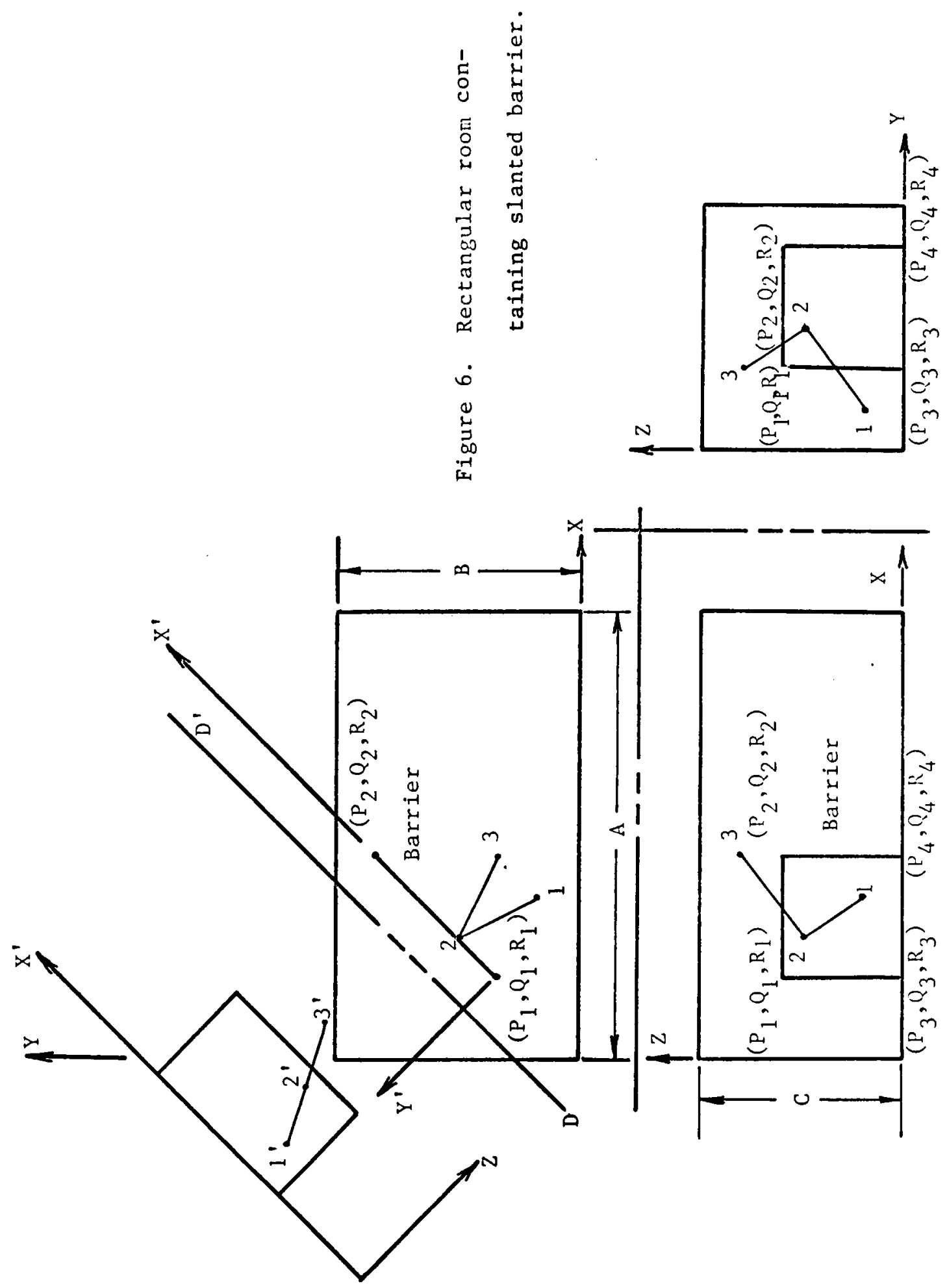




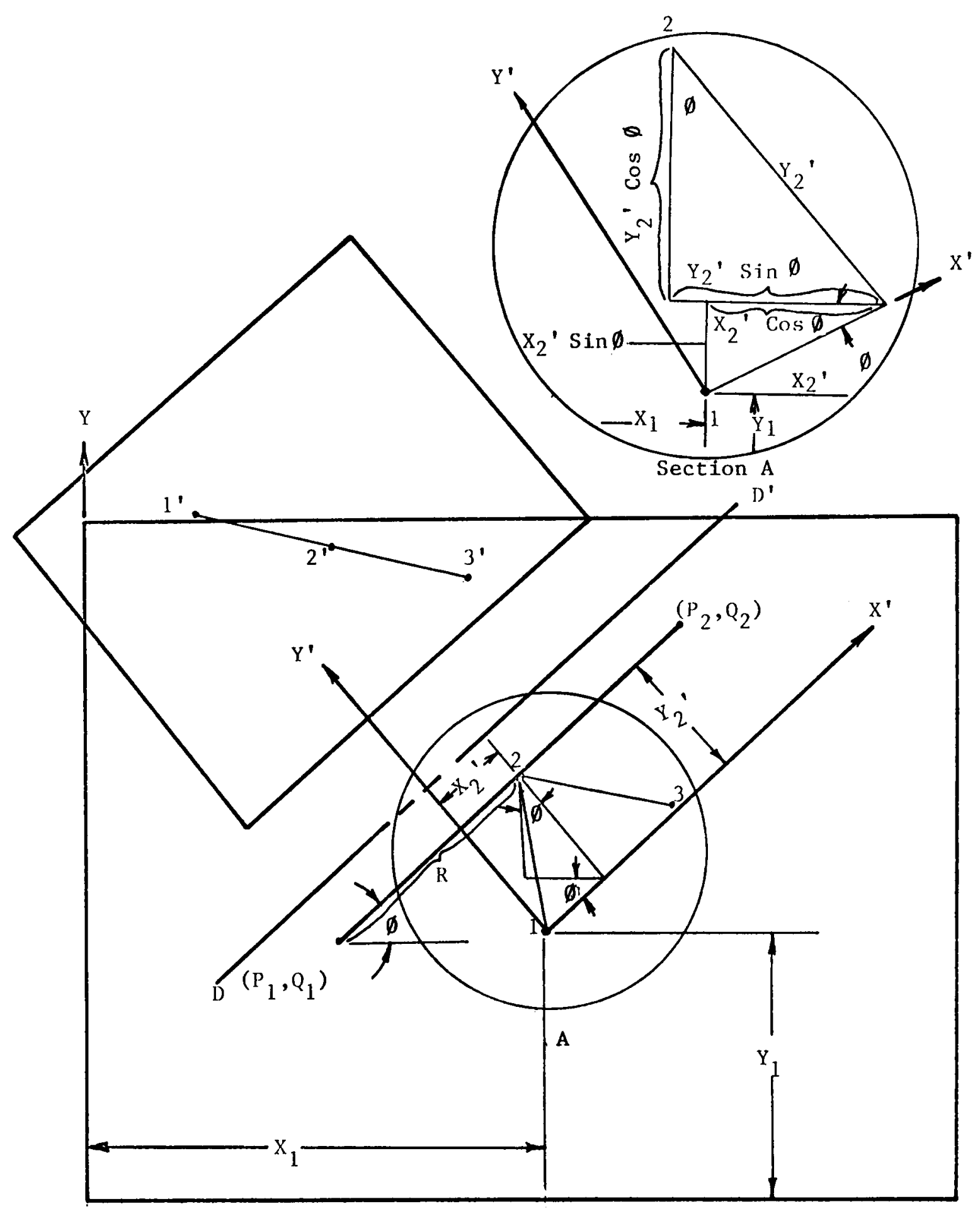

Figure 7. Enlarged view of $\mathrm{X}-\mathrm{Y}$ plane and true size and edge view of slanted barrier shown in Figure 6 . 


$$
\begin{aligned}
& \mathrm{X}_{2}=\mathrm{X}_{1}-\mathrm{Y}_{2}^{\prime} \sin \emptyset+\mathrm{X}_{2}^{\prime} \cos \emptyset \\
& \mathrm{Y}_{2}=\mathrm{Y}_{1}+\mathrm{X}_{2}^{\prime} \sin \emptyset+\mathrm{Y}_{2}^{\prime} \cos \emptyset
\end{aligned}
$$

Section A in Figure 7 shows the distances represented by each term in these equations.

Since the calculations are to be made in the primed system equations (52) and (53) are combined and rearranged to solve for $\mathrm{x}_{2}$ ' and $\mathrm{Y}_{2}{ }^{\prime}$ in terms of the unprimed coordinate axes:

$$
\begin{aligned}
& \mathrm{Y}_{2}{ }^{\prime}=\sin \emptyset\left(\mathrm{X}_{1}-\mathrm{X}_{2}\right)+\cos \emptyset\left(\mathrm{Y}_{2}-\mathrm{Y}_{1}\right) \\
& \mathrm{X}_{2}{ }^{\prime}=\sin \emptyset\left(\mathrm{Y}_{2}-\mathrm{Y}_{1}\right)+\cos \emptyset\left(\mathrm{X}_{2}-\mathrm{X}_{1}\right)
\end{aligned}
$$

The next problem is to determine which, if any, of the coordinates in the primed system are known. Let the end point of the slanted surface with the minimum $X$ dimension be point $\left(P_{1}, Q_{1}\right)$ as shown in Figure 7. The distance from point $\left(P_{1}, Q_{1}\right)$ to point 2 in the figure is defined as " $R$ ". One may write $X_{2}$ and $Y_{2}$ as a function of $\mathrm{R}, \emptyset, \mathrm{P}_{1}$ and $\mathrm{Q}_{1}$;

$$
\begin{aligned}
& \mathrm{X}_{2}=\mathrm{P}_{1}+\mathrm{R} \operatorname{Cos} \emptyset \\
& \mathrm{Y}_{2}=\mathrm{Q}_{1}+\mathrm{R} \operatorname{Sin} \emptyset
\end{aligned}
$$

Upon substitution of $\mathrm{X}_{2}$ and $\mathrm{Y}_{2}$ into equation (54):

$$
\mathrm{Y}_{2}^{\prime}=\operatorname{Sin} \emptyset\left(\mathrm{X}_{1}-\mathrm{P}_{1}-\mathrm{R} \operatorname{Cos} \emptyset\right)+\operatorname{Cos} \emptyset\left(\mathrm{Q}_{1}+\mathrm{R} \operatorname{Sin} \emptyset-\mathrm{Y}_{1}\right)(57)
$$

Equation (57) may be reduced to:

$$
\mathrm{Y}_{2}{ }^{\prime}=\left(\mathrm{X}_{1}-\mathrm{P}_{1}\right) \sin \emptyset+\left(\mathrm{Q}_{1}-\mathrm{Y}_{1}\right) \cos \emptyset
$$


All of the quantities to the right of the equal sign in equation (58) are known, so $\mathrm{Y}_{2}$ ' may be determined.

With $\mathrm{Y}_{2}$ ' known Snells law may be applied in the primed coordinate system to solve for $\mathrm{X}_{2}$ ' using:

$$
\frac{\mathrm{Y}_{3}^{\prime}-\mathrm{Y}_{2}^{\prime}}{\mathrm{X}_{3}^{\prime}-\mathrm{X}_{2}^{\prime}}=\frac{-\left(\mathrm{X}_{2}^{\prime}-\mathrm{X}_{1}^{\prime}\right)}{\mathrm{Y}_{2}^{\prime}-\mathrm{Y}_{1}^{\prime}}
$$

Since the coordinate axes of the primed system have their origin at $\left(\mathrm{X}_{1}, \mathrm{Y}_{1}\right), \mathrm{X}_{1}^{\prime}$ and $\mathrm{Y}_{1}^{\prime}$ are both zero. Upon rearrangement of equation (59) to solve for $x_{2}^{\prime}$ :

$$
X_{2}^{\prime}=\frac{Y_{2}^{\prime}-X_{3}^{\prime}}{Y_{3}^{\prime}-2 Y_{2}^{\prime}}
$$

Once $\mathrm{X}_{2}{ }^{\prime}$ and $\mathrm{Y}_{2}{ }^{\prime}$ are determined, $\mathrm{X}_{2}$ and $\mathrm{Y}_{2}$ may then be found using equations (52) and (53).

The only remaining unknown for a single reflection from a slanted surface is $Z_{2}$. $Z_{2}$ can be found by applying Snell's law to the view in the $X^{\prime}-Z$ axis:

$$
z_{2}=\frac{z_{3} x_{2}^{\prime}+z_{1}\left(x_{3}^{\prime}-x_{2}^{\prime}\right)}{x_{3}}
$$

Thus, all reflection points have been found for the case of a single reflection from a slanted surface.

The analysis becomes more involved when two reflections from slanted surfaces are considered. The possible cases involving two reflections would include reflections from:

1. A slanted surface to a "regular" surface;

2. A regular surface to a slanted surface;

3. Two successive slanted surfaces. 
For cases 1 and 2 the equations are derived using the rotated axis system (primed) used in the previous section and the unrotated axes used in the "regular" room analysis. The equations for a typical "case 1 " ray will be derived.

One of the possible case 1 combinations is a reflection from a slanted wall to an unslanted wall. This case is shown in Figure 8 .

The known values for this case are the coordinates of the source and receiver (points 1 and 4 ) and $Y_{2}^{\prime}$ and $X_{3} \cdot Y_{2}^{\prime}$ can be determined in the same manner as in the single reflection case, i.e., using equation (17) which is a function of $X_{1}, Y_{1}, P_{1}, Q_{1}$ and $\emptyset$, all known quantities. $x_{3}$ is known because it lies in the plane $X=A$.

The following equations can be derived by applying Snell's law to various views in the primed and unprimed coordinate systems. From the $X-Y$ coordinate $p l a n e:$

$$
\frac{X_{4}-X_{3}}{Y_{4}-Y_{3}}=\frac{-\left(X_{3}-X_{2}\right)}{Y_{3}-Y_{2}}
$$

From the $\mathrm{X}-\mathrm{Z}$ coordinate plane:

$$
\frac{z_{2}-z_{3}}{x_{2}-x_{3}}=\frac{-\left(z_{3}-z_{4}\right)}{x_{3}-x_{4}}
$$

From the $Y^{\prime}-\mathrm{Z}$ coordinate plane:

$$
\frac{Y_{3}^{\prime}-Y_{2}^{\prime}}{Z_{3}-Z_{2}}=\frac{-\left(Y_{2}^{\prime}\right)}{Z_{2}-Z_{1}}
$$

and from the $X^{\prime}-Y^{\prime}$ coordinate plane:

$$
\frac{\mathrm{X}_{3}^{\prime}-\mathrm{X}_{2}^{\prime}}{\mathrm{Y}_{3}^{\prime}-\mathrm{Y}_{2}^{\prime}}=\frac{-\left(\mathrm{x}_{2}^{\prime}\right)}{\mathrm{Y}_{2}^{\prime}}
$$



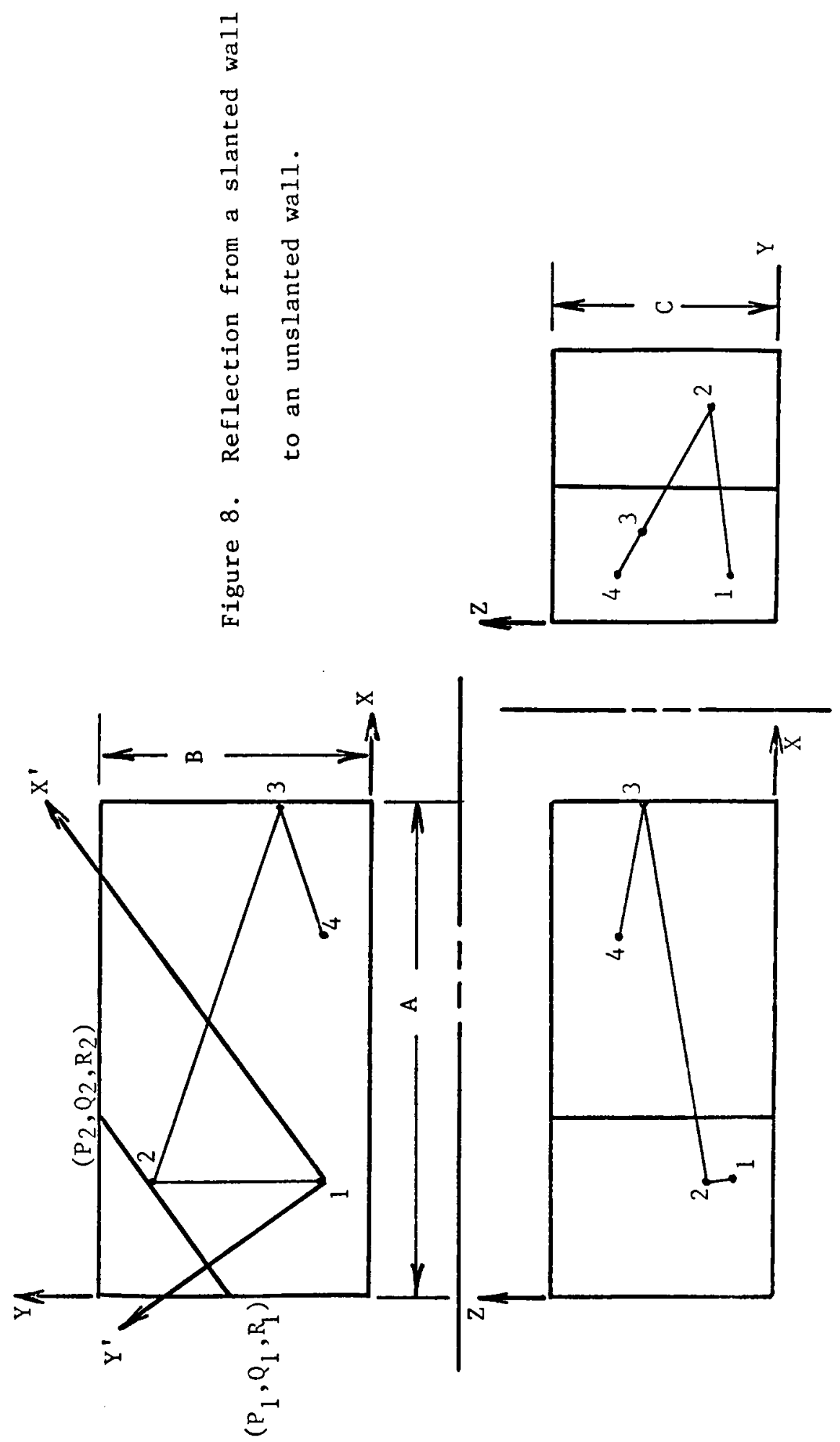
The primed quantities are in the rotated coordinate system. Equations (20) through (23) may be used to solve for $x_{2}$ in the following manner. $Y_{3}$ may be solved for in equation (62):

$$
Y_{3}=\frac{Y_{4} X_{3}+y_{2} X_{4}-Y_{2} X_{3}+Y_{4} X_{2}}{X_{2}+X_{4}-2 X_{3}}
$$

A relationship between $\mathrm{X}$ and $\mathrm{Y}$ on the slanted wall can be derived from its edge view in Figure 8. The equation is of the form:

$$
Y=M X+b
$$

where:

$\mathrm{Y}: \mathrm{Y}$ coordinate of a point on the line (plane)

$X$ : $X$ coordinate of a point on the line

M: Slope of line (plane)

$\mathrm{b}: \quad \mathrm{Y}$ intercept of line (plane)

This equation allows $Y_{2}$ to be expressed as a function of $X_{2}$ in (67a):

$$
\mathrm{Y}_{2}=\mathrm{M \textrm {X } _ { 2 }}+\mathrm{b}
$$

This equation, when substituted into equation 66 , yields

$$
y_{3}=\frac{a_{1} x_{2}+a_{2}}{x_{2}+a_{3}}
$$

where:

$$
\begin{aligned}
& a_{1}=M\left(x_{4}-x_{3}\right)+y_{4} \\
& a_{2}=b\left(x_{4}-x_{3}\right)-y_{4} x_{3} \\
& a_{3}=x_{4}-2 x_{3}
\end{aligned}
$$

To eliminate $\mathrm{Y}_{3}$ another equation for $\mathrm{Y}_{3}$ in terms of $\mathrm{X}_{2}$ must be 
determined. From the transformation equations for the rotated axis equations (54) and (55) we know:

$$
\begin{aligned}
& \mathrm{x}_{2}{ }^{\prime}=\sin \emptyset\left(\mathrm{Y}_{2}-\mathrm{Y}_{1}\right)+\cos \emptyset\left(\mathrm{x}_{2}-\mathrm{X}_{1}\right) \\
& \mathrm{x}_{3}{ }^{\prime}=\sin \emptyset\left(\mathrm{Y}_{3}-\mathrm{Y}_{1}\right)+\cos \emptyset\left(\mathrm{x}_{3}-\mathrm{x}_{1}\right) \\
& \mathrm{Y}_{3}{ }^{\prime}=\sin \emptyset\left(\mathrm{x}_{1}-\mathrm{x}_{3}\right)+\cos \emptyset\left(\mathrm{Y}_{3}-\mathrm{Y}_{1}\right)
\end{aligned}
$$

These equations can be substituted into equation (65). With the additional substitution of equation (67a) an expression for $Y_{3}$ in terms of $\mathrm{x}_{2}$ is determined.

$$
Y_{3}=\frac{E_{1} X_{2}+E_{2}}{E_{3} X_{2}+E_{4}}
$$

where:

$$
\begin{aligned}
& \mathrm{E}_{1}=2 \mathrm{~B}_{1} \mathrm{Y}_{2}^{\prime}+\mathrm{B}_{1} \mathrm{C}_{1} \mathrm{X}_{3}-\mathrm{B}_{1} \mathrm{C}_{3} \\
& \mathrm{E}_{2}=\mathrm{B}_{2}\left(\mathrm{Y}_{2}^{\prime}-\mathrm{C}_{1} \mathrm{X}_{3}-\mathrm{C}_{3}\right)+\mathrm{Y}_{2}^{\prime}\left(\mathrm{B}_{2}+\mathrm{C}_{2} \mathrm{X}_{3}-\mathrm{D}_{1}\right) \\
& \mathrm{E}_{3}=\mathrm{C}_{2} \mathrm{~B}_{1} \\
& \mathrm{E}_{4}=\mathrm{C}_{2} \mathrm{~B}_{2}-\mathrm{C}_{1} \mathrm{Y}_{2}^{\prime} \\
& \mathrm{B}_{1}=-\mathrm{M}^{\prime} \operatorname{Sin} \emptyset-\operatorname{Cos} \emptyset \\
& \mathrm{B}_{2}=\operatorname{Sin} \emptyset\left(\mathrm{Y}_{1}-\mathrm{B}^{\prime}+\mathrm{X}_{1} \operatorname{Cos} \emptyset\right. \\
& \mathrm{C}_{1}=\operatorname{Sin} \emptyset \\
& \mathrm{C}_{2}=-\operatorname{Cos} \emptyset \\
& \mathrm{C}_{3}=\mathrm{Y}_{1} \operatorname{Cos} \emptyset-\mathrm{X}_{1} \operatorname{Sin} \emptyset
\end{aligned}
$$

Equations (68) and (70) can now be equated to solve for $\mathrm{x}_{2}$ :

$$
x_{2}=\frac{-F_{2} \pm \sqrt{F_{2}^{2}-4 F_{1} F_{3}}}{2 F_{1}}
$$


where:

$$
\begin{aligned}
& F_{1}=E_{1}-E_{3} A_{1} \\
& F_{2}=E_{2}+A_{3} E_{1}-E_{3} A_{2}-E_{4} A_{1} \\
& F_{3}=E_{2} A_{3}-E_{4} A_{2}
\end{aligned}
$$

Since $x_{3}$ is known quantity $x_{2}$ can be determined from equation (29). Equations (70) and (67) can then be used to determine $\mathrm{Y}_{3}$ and $\mathrm{Y}_{2}$ respectively. The only remaining unknowns are $z_{2}$ and $z_{3}$.

Equation (63) can be rearranged to solve for $\mathrm{Z}_{2}$ :

$$
Z_{2}=\frac{G_{1} Z_{3}+G_{2}}{G_{3}}
$$

where:

$$
\begin{aligned}
& G_{1}=2 x_{3}-x_{2}-x_{4} \\
& G_{2}=z_{4}\left(x_{2}-x_{3}\right) \\
& G_{3}=x_{3}-x_{4}
\end{aligned}
$$

To eliminate $Z_{2}$ from equation (72) another expression must be found for $Z_{2}$ in terms of $Z_{3}$. Equation (65) contains the unknown quantities $\mathrm{Z}_{2}$ and $\mathrm{Z}_{3} \cdot \mathrm{Y}_{2}$ ' is known from equation (58), and $\mathrm{Y}_{3}{ }^{\prime}$ can be determined by equation $(69 c)$. Equation (65) can be rearranged to solve for $z_{2}$ in terms of $Z_{3}$ :

$$
\mathrm{Z}_{2}=\mathrm{H}_{1}-\mathrm{Y}_{2}^{\prime} \mathrm{Z}_{3}
$$

where:

$$
\begin{aligned}
& \mathrm{H}_{1}=\mathrm{Z}_{1}\left(\mathrm{Y}_{3}^{\prime}-\mathrm{Y}_{2}^{\prime}\right) \\
& \mathrm{H}_{2}=\mathrm{Y}_{3}^{\prime}-2 \mathrm{Y}_{2}^{\prime}
\end{aligned}
$$


Equations (72) and (73) can now be equated to solve for $Z_{3}$ :

$$
\mathrm{Z}_{3}=\frac{\mathrm{G}_{3} \mathrm{H}_{1}-\mathrm{H}_{2} \mathrm{G}_{2}}{\mathrm{G}_{1} \mathrm{H}_{2}-\mathrm{G}_{3} \mathrm{Y}_{2}}
$$

Either equation (72) or (73) can now be used to solve for $Z_{3}$.

The dimensions for the other slanted reflection combinations follow the same pattern. The equations are as follows:

Case 2 - slanted wall to regular wall perpendicular to Y-axis:

$$
\begin{aligned}
& \mathrm{Y}_{2}^{\prime}=(\operatorname{Cos} \emptyset)\left(\mathrm{Y}_{1}-\mathrm{Q}_{1}\right)-(\operatorname{Sin} \emptyset)\left(\mathrm{X}_{1}-\mathrm{P}_{1}\right) \\
& \mathrm{A}_{1}=\mathrm{Y}_{4}-\mathrm{Y}_{3}+\mathrm{M}_{4} \\
& \mathrm{~A}_{2}=\mathrm{X}_{4}\left(\mathrm{~b}-\mathrm{Y}_{3}\right) \\
& \mathrm{A}_{3}=\mathrm{b}-2 \mathrm{Y}_{3}+\mathrm{Y}_{4} \\
& \mathrm{~B}_{1}=-\mathrm{M} \operatorname{Sin} \emptyset-\operatorname{Cos} \emptyset \\
& \mathrm{B}_{2}=-(\operatorname{Sin} \emptyset)\left(\mathrm{b}-\mathrm{Y}_{1}\right)+\mathrm{X}_{1} \operatorname{Cos} \emptyset \\
& \mathrm{C}_{1}=\mathrm{Sin} \emptyset \\
& \mathrm{C}_{2}=-\operatorname{Cos} \emptyset \\
& \mathrm{C}_{3}=\mathrm{Y}_{1} \operatorname{Cos} \emptyset-\mathrm{X}_{1} \operatorname{Sin} \emptyset \\
& \mathrm{D}_{1}=\mathrm{Y}_{1} \operatorname{Sin} \emptyset+\mathrm{X}_{1} \operatorname{Cos} \emptyset \\
& \mathrm{E}_{1}=2 \mathrm{~B}_{1} \mathrm{Y}_{2}{ }^{\prime}-\mathrm{B}_{1} \mathrm{C}_{2} \mathrm{Y}_{3}-\mathrm{B}_{1} \mathrm{C}_{3} \\
& \mathrm{E}_{2}=\mathrm{B}_{2}\left(\mathrm{Y}_{2}^{\prime}-\mathrm{C}_{2} \mathrm{Y}_{3}-\mathrm{C}_{3}\right)+\mathrm{Y}_{2}{ }^{\prime}\left(\mathrm{C}_{1} \mathrm{Y}_{3}-\mathrm{D}_{1} \mathrm{~B}_{2}\right) \\
& \mathrm{E}_{3}=\mathrm{C}_{1} \mathrm{~B}_{1} \\
& \mathrm{E}_{4} \mathrm{C}_{1} \mathrm{~B}_{2}+\mathrm{C}_{2} \mathrm{Y}_{2}^{\prime}
\end{aligned}
$$




$$
\begin{aligned}
& F_{1}=M E_{1}-E_{3} A_{1} \\
& F_{2}=A_{3} E_{1}+M E_{2}-E_{3} A_{2} \\
& F_{3}=E_{2} A_{3}-E_{4} A_{2} \\
& x_{2}=\frac{-F_{2} \pm \sqrt{F_{2}{ }^{2}-4 F_{1} F_{3}}}{2 F_{1}} \\
& \mathrm{Y}_{2}=\mathrm{MX}_{2}+\mathrm{b} \\
& X_{3}=\frac{E_{1} X_{2}+E_{2}}{E_{3} X_{2}+E_{4}} \\
& \mathrm{G}_{1}=\mathrm{x}_{2}-\mathrm{x}_{4} \\
& \mathrm{G}_{2}=\mathrm{z}_{4}\left(\mathrm{x}_{3}-\mathrm{x}_{2}\right) \\
& \mathrm{G}_{3}=\mathrm{x}_{3}-\mathrm{x}_{4} \\
& \mathrm{Y}_{3}^{\prime}=\mathrm{C}_{1} \mathrm{X}_{3}+\mathrm{C}_{2} \mathrm{Y}_{3}+\mathrm{C}_{3} \\
& \mathrm{H}_{1}=\mathrm{Z}_{1}\left(\mathrm{Y}_{3}{ }^{\prime}-\mathrm{Y}_{2}{ }^{\prime}\right) \\
& \mathrm{H}_{2}=\mathrm{Y}_{3}^{\prime}-2 \mathrm{Y}_{2}{ }^{\prime} \\
& \mathrm{z}_{3}=\frac{\mathrm{G}_{3} \mathrm{H}_{1}-\mathrm{H}_{2} \mathrm{G}_{2}}{\mathrm{G}_{1} \mathrm{H}_{2}+\mathrm{G}_{3} \mathrm{Y}_{2}}, \\
& \mathrm{Z}_{2}=\frac{\mathrm{H}_{1}-\mathrm{Y}_{2}^{\prime} \mathrm{Z}_{3}}{\mathrm{H}_{2}}
\end{aligned}
$$

Case 3 - slanted plane to ceiling or floor surfaces:

$$
\begin{aligned}
& \mathrm{X}_{4}^{\prime}=-(\operatorname{Sin} \emptyset)\left(\mathrm{Y}_{4}-\mathrm{Y}_{1}\right)-(\operatorname{Cos} \emptyset)\left(\mathrm{X}_{4}-\mathrm{X}_{1}\right) \\
& \mathrm{Y}_{4}^{\prime}=-(\operatorname{Cos} \emptyset)\left(\mathrm{Y}_{4}-\mathrm{Y}_{1}\right)-(\operatorname{Sin} \emptyset)\left(\mathrm{X}_{1}-\mathrm{X}_{4}\right) \\
& \mathrm{Y}_{2}^{\prime}=-(\operatorname{Cos} \emptyset)\left(\mathrm{Q}_{1}-\mathrm{Y}_{1}\right)-(\operatorname{Sin} \emptyset)\left(\mathrm{X}_{1}-\mathrm{P}_{1}\right)
\end{aligned}
$$




$$
\begin{aligned}
& \mathrm{X}_{2}^{\prime}=\frac{\mathrm{Y}_{2}^{\prime} \mathrm{X}_{4}^{\prime}}{2 \mathrm{Y}_{2}^{\prime}-\mathrm{Y}_{4}^{\prime}} \\
& \mathrm{x}_{2}=\mathrm{x}_{1}-\mathrm{x}_{2}{ }^{\prime} \cos \emptyset+\mathrm{Y}_{2}^{\prime} \sin \emptyset \\
& \mathrm{Y}_{2}=\mathrm{Y}_{1}-\mathrm{X}_{2}^{\prime} \sin \emptyset-\mathrm{Y}_{2}^{\prime} \operatorname{Cos} \emptyset \\
& A_{1}=-\sin \emptyset \\
& A_{2}=-\operatorname{Cos} \emptyset \\
& \mathrm{A}_{3}=\mathrm{Y}_{1} \sin \emptyset+\mathrm{X}_{1} \operatorname{Cos} \emptyset \\
& \mathrm{B}_{1}=\mathrm{Y}_{4}-\mathrm{Y}_{2} \\
& \mathrm{~B}_{2}=\mathrm{X}_{4} \mathrm{Y}_{2}-\mathrm{X}_{2} \mathrm{Y}_{4} \\
& \mathrm{~B}_{3}=\mathrm{x}_{4}-\mathrm{x}_{2} \\
& \mathrm{C}_{1}=\mathrm{A}_{2} \mathrm{Z}_{1} \\
& c_{2}=x_{2}^{\prime} z_{3}+z_{1}\left(A_{3}-x_{2}^{\prime}\right) \\
& c_{3}=-A_{1} z_{1} \\
& D_{1}=B_{3} C_{1}-C_{3} B_{1} \\
& D_{2}-B_{3} C_{2}-B_{2} C_{3} \\
& D_{3}=A_{1} B_{1}+B_{3} A_{2} \\
& D_{4}=A_{1} B_{2}+B_{3} A_{3} \\
& E_{1}=2 z_{3}-z_{4} \\
& E_{2}=-z_{3}\left(x_{2}+x_{4}+z_{4} x_{2}\right) \\
& F_{1}=D_{1}-E_{1} D_{3}
\end{aligned}
$$




$$
\begin{aligned}
& F_{2}=D_{2}-D_{1} X_{4}-E_{1} D_{4}-E_{2} D_{3} \\
& F_{3}=-D_{2} X_{4}-E_{2} D_{4} \\
& X_{3}=\frac{-F_{2} \pm \sqrt{F_{2}^{2}-4 F_{1} F_{3}}}{2 F_{1}} \\
& Y_{3}=\frac{B_{1} X_{3}+B_{2}}{B_{3}} \\
& Z_{2}=\frac{x_{3} D_{1}+D_{2}}{X_{3} D_{3}+D_{4}}
\end{aligned}
$$

Case 4 - reflections from two slanted planes perpendicular to same plane:

$$
\begin{aligned}
& \mathrm{Y}_{2}^{\prime}=-\operatorname{Cos} \emptyset_{1}\left(Q_{1}-\mathrm{Y}_{1}\right)-\sin \emptyset_{1}\left(\mathrm{X}_{1}-\mathrm{P}_{1}\right) \\
& \mathrm{Y}_{3}^{\prime}=-\cos \emptyset_{2}\left(Q_{4}-\mathrm{Y}_{4}\right)-\sin \emptyset_{2}\left(\mathrm{X}_{4}-\mathrm{P}_{4}\right) \\
& \mathrm{A}_{1}=-\mathrm{M}_{2} \sin \emptyset_{1}-\cos \emptyset_{1} \\
& \mathrm{~A}_{2}=-\sin \emptyset_{1}\left(\mathrm{M}_{2}-\mathrm{Y}_{1}\right)+\mathrm{X}_{1} \cos \emptyset_{1} \\
& \mathrm{~B}_{1}=-\mathrm{M}_{2} \operatorname{Cos} \emptyset_{1}+\sin \emptyset_{1} \\
& \mathrm{~B}_{2}=-\operatorname{Cos} \emptyset_{1}\left(\mathrm{M}_{2}-\mathrm{Y}_{1}-\mathrm{X}_{1} \sin \emptyset_{1}\right. \\
& \mathrm{C}_{1}=-\mathrm{M}_{1} \sin \emptyset_{1}-\operatorname{Cos} \emptyset_{1} \\
& \mathrm{C}_{2}=-\sin \emptyset_{1}\left(\mathrm{M}_{1}-\mathrm{Y}_{1}\right)+\mathrm{X}_{1} \operatorname{Cos} \emptyset_{1} \\
& \mathrm{D}_{1}=2 \mathrm{C}_{1} \mathrm{Y}_{2}^{\prime}-\mathrm{C}_{1} \mathrm{~B}_{2} \\
& \mathrm{D}_{2}=2 \mathrm{C}_{2} \mathrm{Y}_{2}^{\prime}-\mathrm{B}_{2} \mathrm{C}_{2}-\mathrm{Y}_{2}^{\prime} \mathrm{A}_{2} \\
& \mathrm{D}_{3}=\mathrm{B}_{1} \mathrm{C}_{1} \\
& \mathrm{D}_{4}=\mathrm{B}_{1} \mathrm{C}_{2}+\mathrm{A}_{1} \mathrm{Y}_{2}^{\prime}
\end{aligned}
$$


41

$$
E_{1}=-M_{1} \sin \emptyset_{2}-\cos \emptyset_{2}
$$$$
E_{2}=-\left(\operatorname{Sin} \emptyset_{2}\right)\left(M_{1}-Y_{4}+X_{4}\right) \operatorname{Cos} \emptyset_{2}
$$$$
F_{1}=-M_{2} \sin \emptyset_{2}-\cos \emptyset_{2}
$$$$
F_{2}=-\left(\sin \emptyset_{2}\right)\left(M_{2}-Y_{4}\right)+X_{4} \cos \emptyset_{2}
$$$$
G_{1}=-M_{1} \cos \emptyset_{2}+\sin \emptyset_{2}
$$

$$
\mathrm{G}_{2}=-\left(\operatorname{Cos} \emptyset{ }_{2}\right)\left(\mathrm{M}_{1}-\mathrm{Y}_{4}\right)-\mathrm{X}_{4} \sin \emptyset_{2}
$$$$
H_{1}=-F_{2} G_{1}-Y_{3}^{\prime \prime} E_{1}
$$

$$
\mathrm{H}_{2}=2 \mathrm{~F}_{2} \mathrm{Y}_{3}{ }^{\prime}-\mathrm{F}_{2} \mathrm{G}_{2}-\mathrm{Y}_{3}{ }^{\prime \prime} \mathrm{E}_{2}
$$$$
H_{3}=F_{1} G_{1}
$$

$$
\begin{aligned}
& \mathrm{H}_{4}=\mathrm{F}_{1} \mathrm{G}_{2}-2 \mathrm{~F}_{1} \mathrm{Y}_{3}{ }^{\prime} \\
& \mathrm{S}_{1}=\mathrm{H}_{1} \mathrm{D}_{3}-\mathrm{H}_{3} \mathrm{D}_{1} \\
& \mathrm{~S}_{2}=\mathrm{H}_{1} \mathrm{D}_{4}+\mathrm{H}_{2} \mathrm{D}_{3}-\mathrm{H}_{3} \mathrm{D}_{2}-\mathrm{H}_{4} \mathrm{D}_{1} \\
& \mathrm{~S}_{3}=\mathrm{H}_{2} \mathrm{D}_{4}-\mathrm{H}_{4} \mathrm{D}_{2} \\
& \mathrm{X}_{2}=\frac{-\mathrm{S}_{2} \pm \sqrt{\mathrm{S}_{2}{ }^{2}-4 \mathrm{~S}_{1} \mathrm{~S}_{3}}}{2 \mathrm{~S}_{1}} \\
& \mathrm{Y}_{2}=\mathrm{M}_{1} \mathrm{X}_{2}+\mathrm{b}_{1} \\
& \mathrm{X}_{3}=\frac{\mathrm{H}_{1} \mathrm{X}_{2}+\mathrm{H}_{2}}{\mathrm{H}_{3} \mathrm{X}_{2}+\mathrm{H}_{4}} \\
& \mathrm{Y}_{3}=\mathrm{M}_{2} \mathrm{X}_{3}+\mathrm{b}_{2} \\
& \mathrm{X}_{3}{ }^{\prime}=A_{1} \mathrm{X}_{3}+\mathrm{A}_{2}
\end{aligned}
$$




$$
\begin{aligned}
& \mathrm{x}_{2}^{\prime}=\mathrm{c}_{1} \mathrm{x}_{2}+\mathrm{c}_{2} \\
& \mathrm{Y}_{3}^{\prime}=\mathrm{B}_{1} \mathrm{X}_{3}+\mathrm{B}_{2} \\
& \mathrm{~T}_{1}=\mathrm{x}_{3}^{\prime} \mathrm{z}_{1}-\mathrm{x}_{2}^{\prime} \mathrm{z}_{1} \\
& \mathrm{x}_{3}{ }^{\prime \prime}=\mathrm{F}_{1} \mathrm{x}_{3}+\mathrm{F}_{2} \\
& x_{2}^{\prime \prime}=E_{1} x_{2}+E_{2} \\
& \mathrm{~T}_{2}=\mathrm{x}_{3}{ }^{\prime \prime} \mathrm{z}_{4}-\mathrm{x}_{2}{ }^{\prime \prime} \mathrm{z}_{4} \\
& z_{3}=\frac{x_{3}^{\prime} T_{2}-T_{1} x_{3}^{\prime \prime}}{x_{2}^{\prime} x_{3}^{\prime \prime}-x_{3}^{\prime} x_{2}^{\prime \prime}} \\
& z_{2}=\frac{x_{2}^{\prime \prime} z_{3}+T_{2}}{x_{3}{ }^{\prime \prime}}
\end{aligned}
$$

In the preceding equations the initial coordinate is determined by the quadratic equation (equations (71), (92), (127), (155)). Both roots were not possible solutions so in all cases one of them was extraneous. A check is made in the program to discard the extraneous root.

The previous procedures were applied in an attempt to solve for three reflections, involving at least one slanted surface. The resulting equations contained second and third order terms and multiples of them which could not be reduced to the form used for two reflections. Because of the algebraic problems involved with these equations, they were not solved. Iteration was considered but was not attempted in this work. If a solution were found the presence of third order terms indicates that additional methods of determining the 
correct root would be necessary, therefore increasing computational time. Hence, ray tracing equations involving slanted surfaces were derived for only one and two reflections. 


\subsection{APPLICATION OF RAY TRACING EQUATIONS TO COMPUTER PROGRAM}

\subsection{Calculation of Rays}

Since the ray tracing equations determine the coordinate locations of the reflection points, a coordinate system must be established for each room considered. To assure all coordinate points are positive the room is located in the first quadrant.* The coordinates of the source, the receiver and all of the corners of each wall are the only input necessary for the calculation of the rays.

The coordinates of each wall corner define a plane in which the wall lies. The program determines the equation of all the planes containing a wall $(X=A, Y=0$, etc.) and uses these values in the ray tracing equations to determine all the possible reflections.

\subsection{Validity of Calculated Rays}

For mathematical reasons the walls in a room were stored as infinite planes, with the equation of the plane known. The ray tracing equations used this information to calculate reflection points. Figure 9 shows a wall and the infinite plane which contains the wall. Because the ray tracing equations calculate reflection points based on infinite planes and not walls with finite boundaries the possibility exists that a calculated reflection point may occur outside of a wall boundary.

*A complete users guide in Appendix A details the input procedure. 


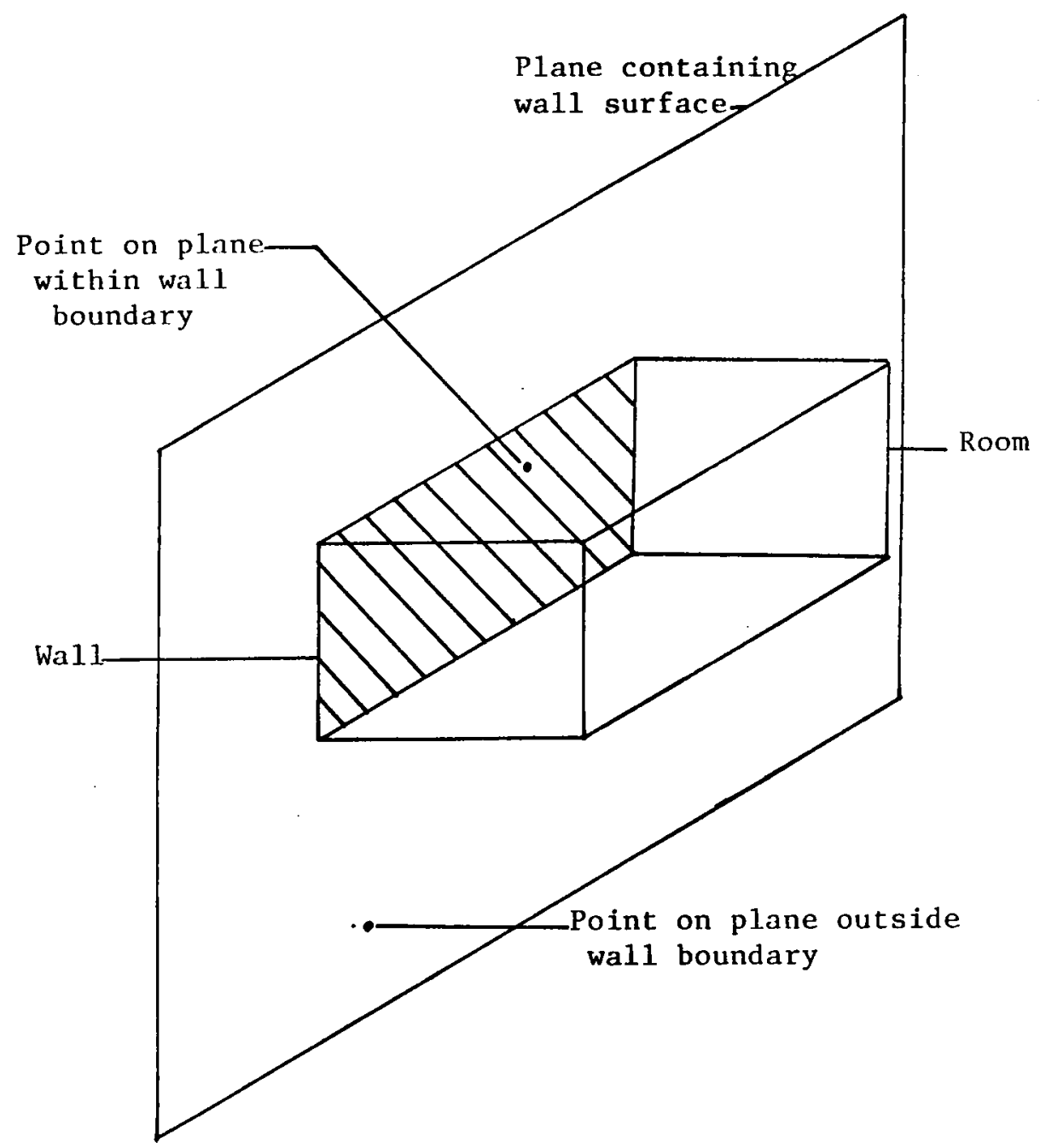

Figure 9. Illustration of wall within a plane concept used in computer program. 
Examples of four ray paths from a point $S$ (source) to a point $R$ (receiver), within a room bounded by ABCDEFGHA are shown in Figure 10 . These examples show some of the rays which would be calculated because reflections are determined from infinite planes. Quite obviously it shows examples of certain ray paths and reflection points that do not occur in practice. The only valid ray path is S-2-R. The others illustrate the possible violations that may occur. Thus it is necessary to include in the program a procedure to eliminate the invalid rays.

To eliminate the invalid rays as quickly as possible each ray path was divided into segments. Then, each segment was considered individually. If a segment is determined to be invalid then the entire ray path is invalid without the need of checking the remaining segments. For example, in Figure 10 ray path $S-2-R$ is a single reflection path which consists of two line segments $S-2$ and $S-R$.

The ray segment method is used as follows. Ray segment S-2, of ray path $S-2-R$, does not intersect any walls except at the desired reflection point 2 . In addition, the reflection point 2 occurs within the wall boundary. Thus, segment $\mathrm{S}-2$ is valid. Similar arguments apply to segment $2-R$. Therefore, segment $2-R$ is a valid segment, and, hence, ray path $S-2-R$ is a valid ray.

Reflection path $\mathrm{S}^{-} 2^{\prime}-\mathrm{R}$ is an example of an invalid ray path. Point $2^{\prime}$ was calculated as a reflection point since wall C-D was considered infinite in extent. Segment S-2' intersects no planes within wall boundaries. Since ${\mathrm{S}-2^{\prime}}^{\prime}$ is not a direct ray path $\mathrm{S}-2^{\prime}$ is an invalid ray. 


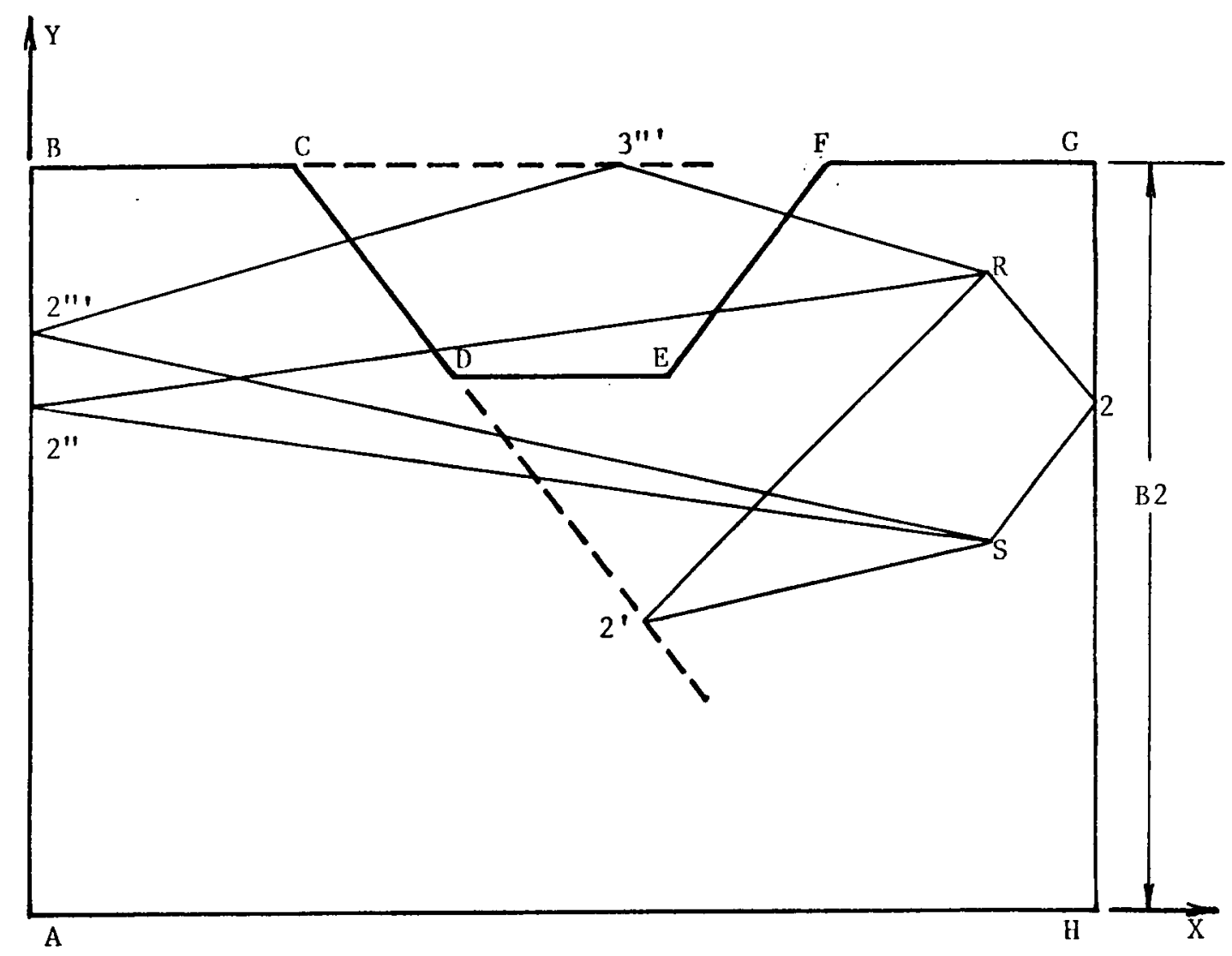

Figure 10. Examples of valid and invalid rays. 
Reflection path $\mathrm{S}-2{ }^{\prime}$ '-R is also invalid. Ray segment $\mathrm{S}-2{ }^{\prime}$ ' is valid by the same reasoning as for ray segment S-2. However, segment 2 ''-R does not qualify as a valid segment. This segment intersects two wall planes within wall boundaries and is therefore invalid.

Reflection path S-2''-3'''-R is an example of a ray path that fails both of the previously discussed criteria. Segment 2 ' '- 3 ''' intersects wall plane C-D within the wall boundary, and reflection point $3^{\prime \prime \prime}$ does not lie within the boundaries of $B C$ or FG, although it lies on the plane common to both of them.

To eliminate invalid ray paths such as $1-2^{\prime}-3$ and $1-2^{\prime \prime}-3$ a subprogram was developed to check each ray path after it had been calculated. The flow chart in Figure 11 indicates the steps taken to check each segment. The subprogram divided each ray path into ray segments and compared them one at a time with all of the walls in the room. The segments were checked to see if an invalid ray had been calculated.

The subprogram called on several other subprograms to determine if the ray was valid. As discussed earlier, a program calculated the equation of each wall plane. The equation of a line containing the ray segment in question was also computed by a subprogram. With these two equations the plane and line were checked to see if they were parallel. If they were parallel it was necessary to determine if the line segment lay within the plane or whether it was a finite distance from the plane. Subprograms performed each of these operations. 


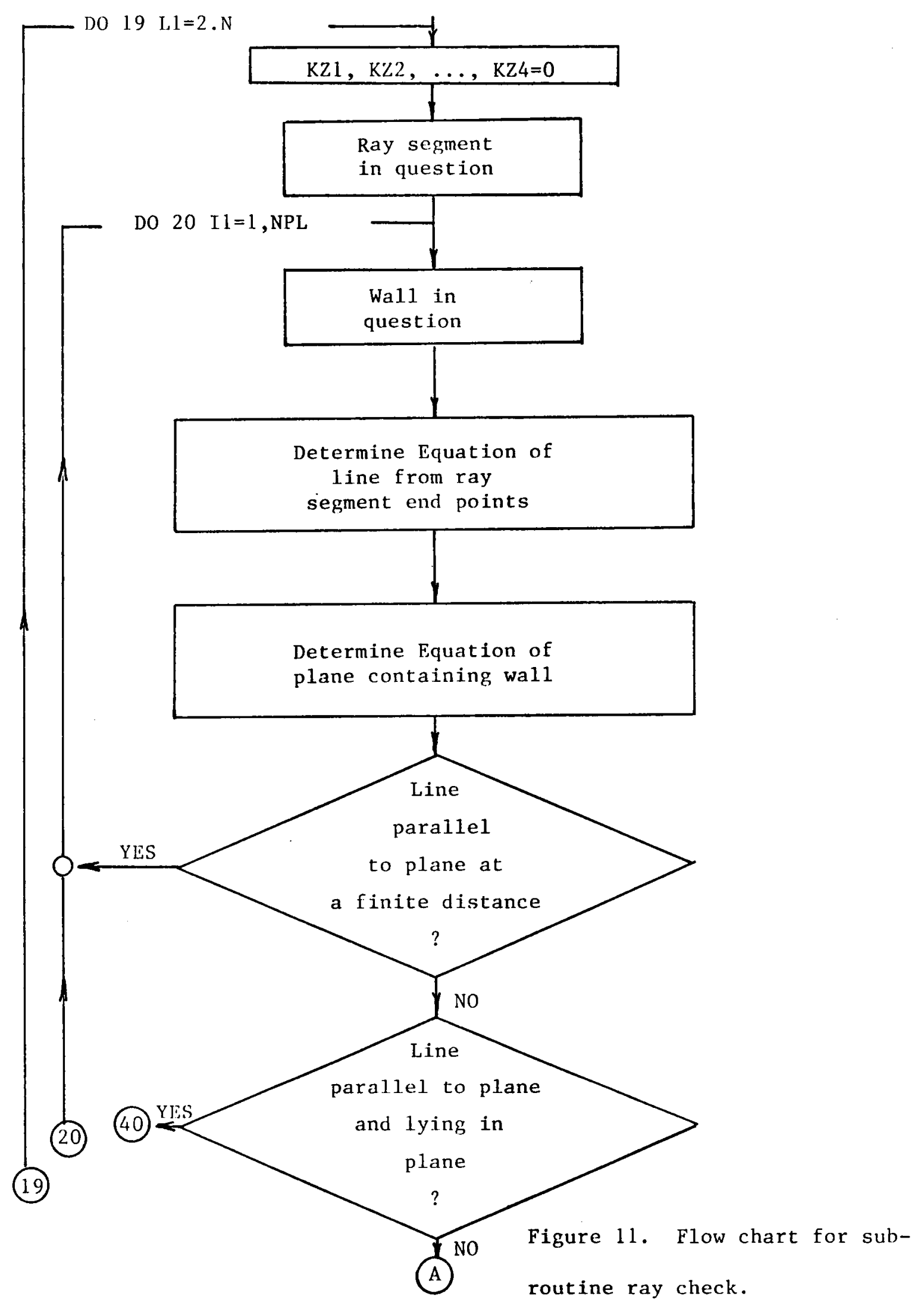




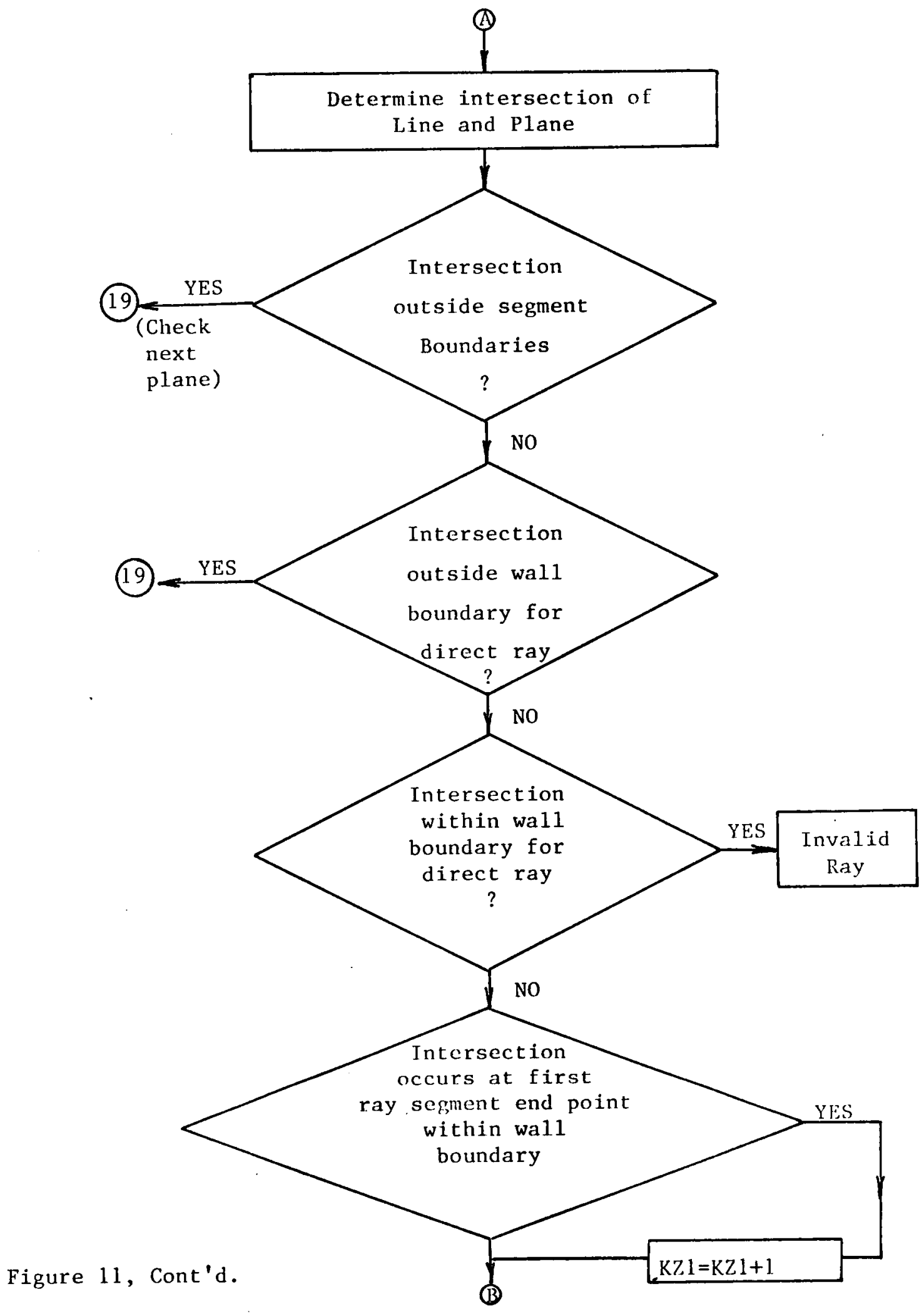




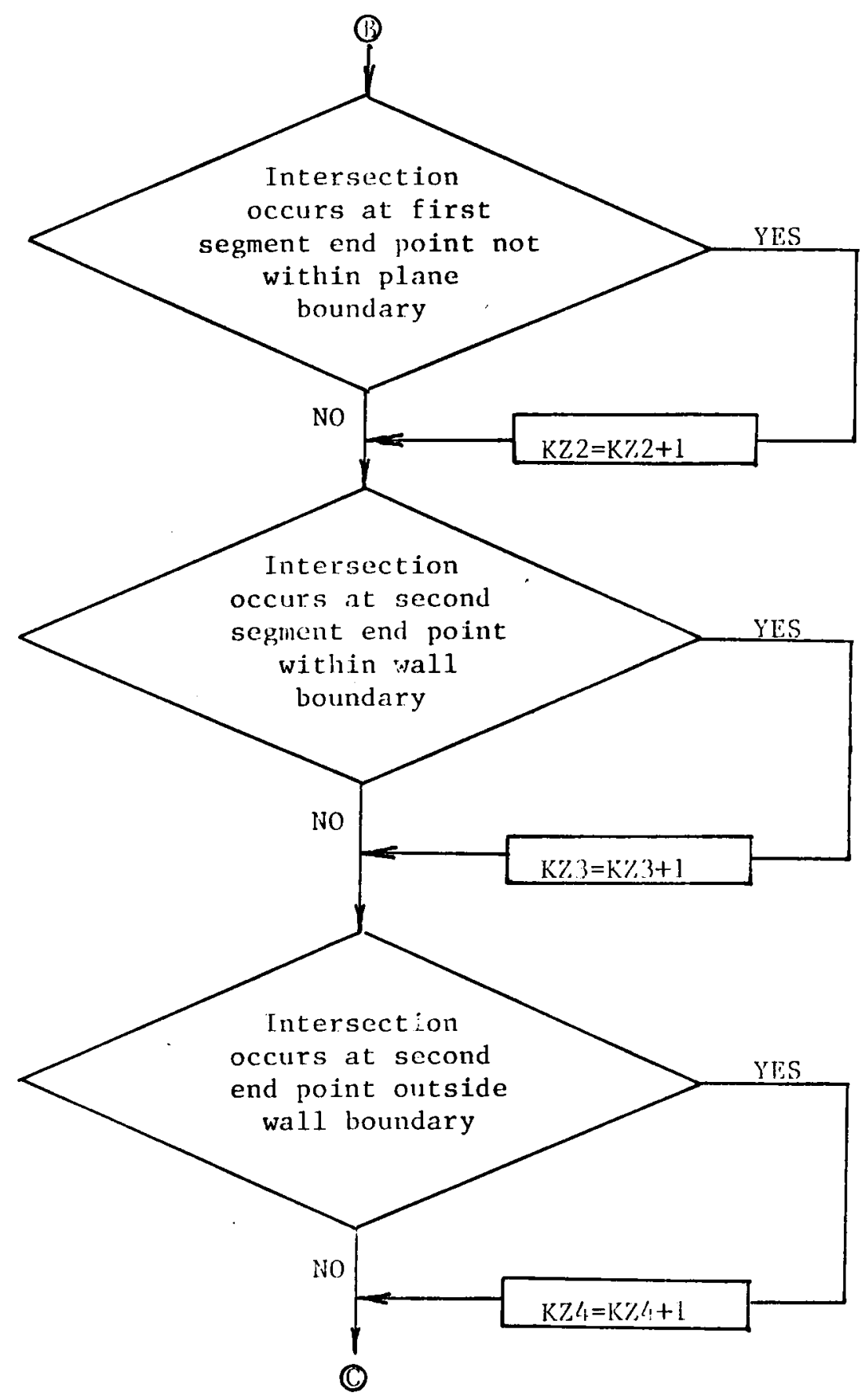

Figure 11, Cont'd. 


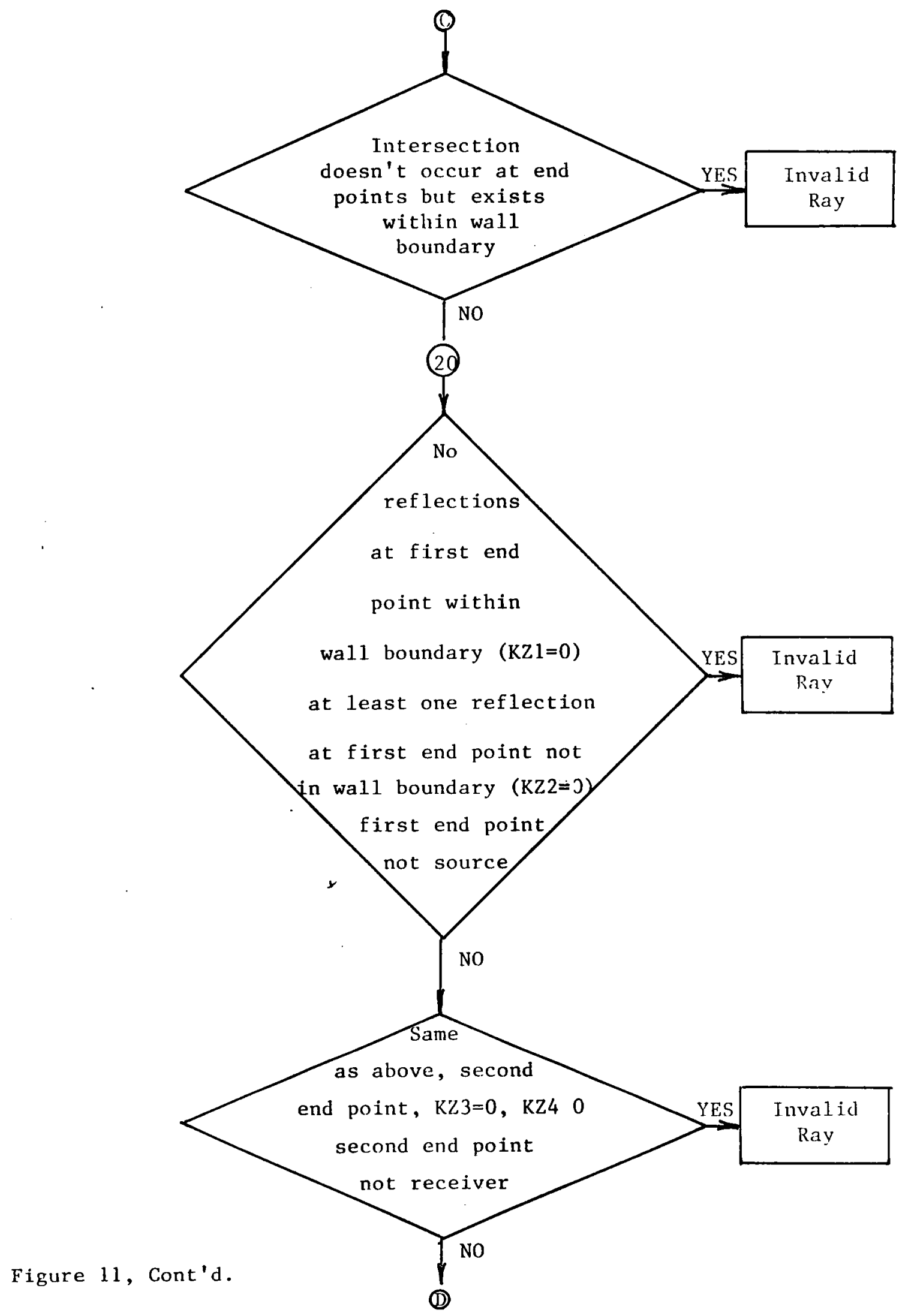



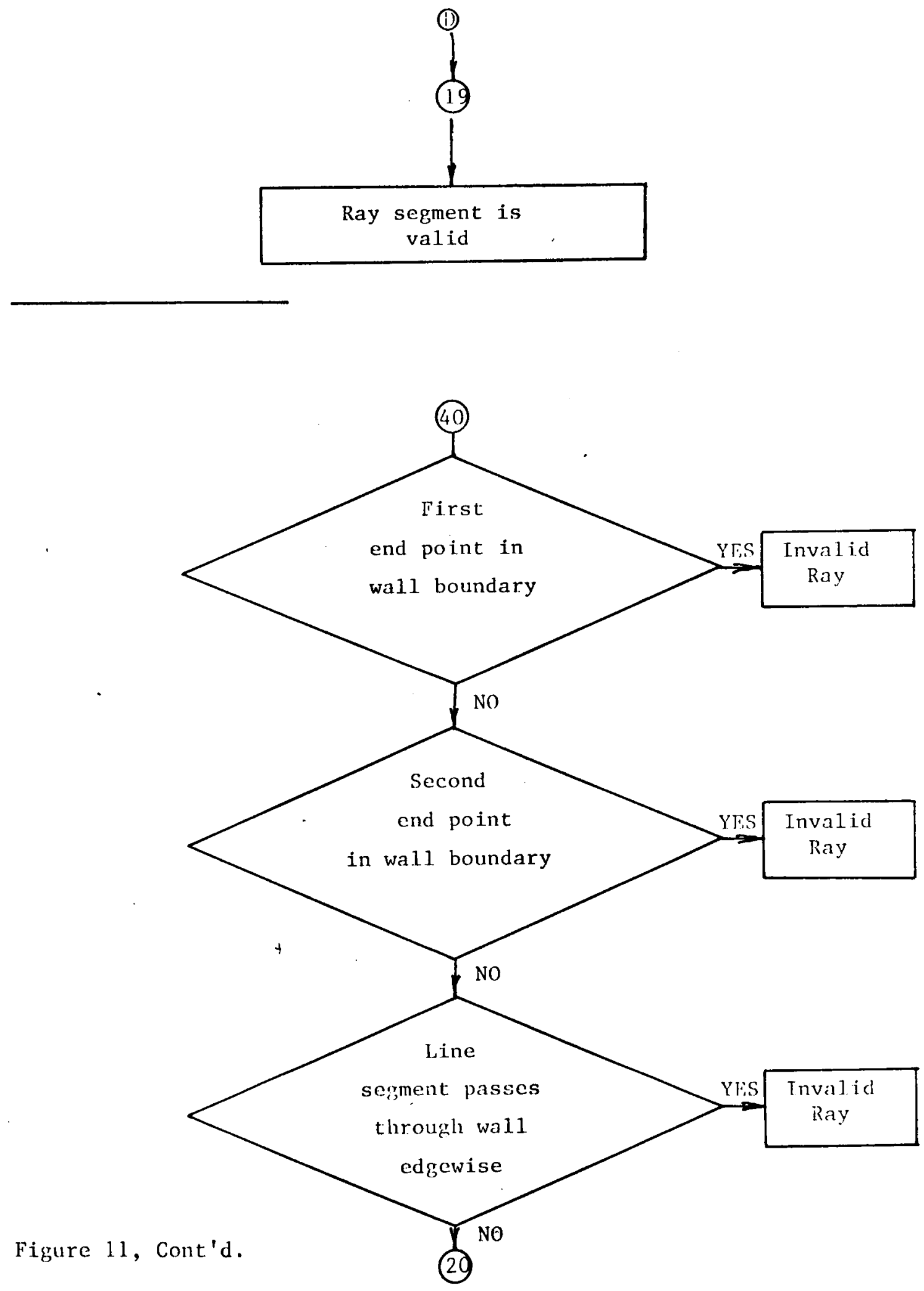
If the line and plane were not parallel then their intersection point was determined. This point was then checked to see if it:

1. Lay in the line segment.

2. Coincided with either line segment end point.

If the intersection point was not between the line segment end points the intersection was not of concern and a new wall was checked. If condition 2 were satisfied then the intersection point was checked to see if it:

1. Lay within a wall boundary.

2. Lay outside a wall boundary.

Intuitively one might think that if the intersection point between the line and the plane lay outside a particular wall boundary an invalid ray 1ike $S-2^{\prime}-R$ as illustrated in Figure 10 had occurred. However, there might be more than one wall in a single plane. Figure 12 illustrates this point. Reflection path S-2-R is a valid ray path. Each segment is checked with all walls. Segment S-2 would be valid when checked with wall B-C. But, if wall F-G and B-C lay in the same plane segment S-2 would be invalid when checked with wall F-G. Point 2, although intersecting the plane at an end point, does not lie within the wall boundary F-G. So, instead of eliminating immediately rays which intersected a plane outside of a wall boundary, a count was made to see how many times the intersection between the wall plane and the line occurred at a ray segment end point both inside and outside of wall boundaries. In the flow chart in Figure 11 the $\mathrm{KZ}$ values 


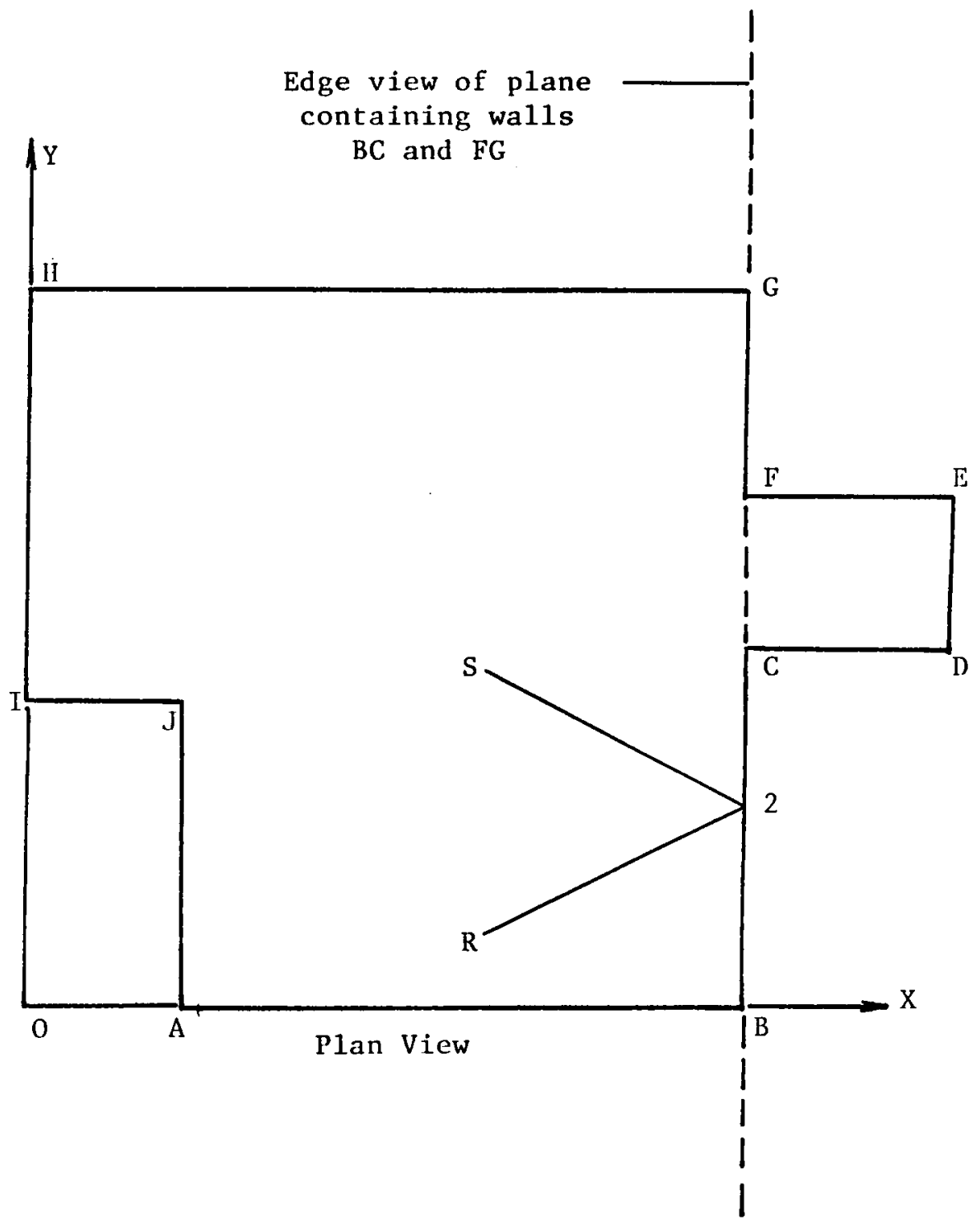

Figure 12. Illustration of case where intersection point between line and plane lying outside a wall boundary (wall F-G) does not imply invalid ray. 
were the counting variables for this. If an intersection occurred at an end point and within a wall boundary then KZl was incremented by one. If the intersection occurred outside of a wall boundary at a segment endpoint, KZ2 was incremented by one. This procedure was followed for both ray segment endpoints. KZ3 and KZ4 were incremented in a similar manner for the other ray segment end point. A given ray segment was invalid if no line-plane intersections had occurred at a ray segment end point and within any wall boundary (KZ1 or KZ3 equal to zero) and if at least one time a line-plane intersection occurred at a ray segment end point outside of a plane boundary (KZ2 or KZ4 greater than zero).

The validity of ray path S-2-R in Figure 12 can be determined as follows. The intersection point (2) occurred at wall B-C within the wall boundary. Since an intersection had occurred at the first segment end point and within a boundary, KZ3 was one. Ray segment S-2 also intersected the plane containing wall FG but outside of the boundaries of FG. Therefore, KZ4, was one. Since an intersection had occurred within a boundary at an end point $(K Z 3=1)$ the ray was valid.

Those walls that were intersected by a line between segment end points and within the wall boundary were discarded immediately. Ray path $\mathrm{S}-2{ }^{\prime}$ '-R in Figure 10 illustrates this case.

Ray paths were valid if each of its segments passed all of the aforementioned tests. 
An interesting problem which arose in making these checks was determining if a ray segment end point on a given plane lay outside or inside the wall boundary. Figure 13 shows an example wall with three possible ray segment end points $A, B$ and $C$.

Figure 13 is a two dimensional view because it is known that points A, B, C lay on the plane containing wall 1-2-3-4-5-6-7-1 because they were calculated assuming a reflection from that plane. The only requirement for this analysis is that the wall appear as an enclosed boundary and not as an edge view.

A reference point (Pref) is chosen outside the wall boundary and lying in the given plane. A reference line segment is constructed by joining Pref and one of the three points in question. The number of times this segment crosses the wall boundary will tell us whether the point lays inside the wall boundary. Segment Pref-C does not cross the wall boundary at all. Therefore, point $\mathrm{C}$ does not lie in the wall boundary. Segment Pref-A crosses the wall boundary one time while segment Pref-B crosses the wall boundary four times. By applying this technique to other points it can be seen that zero or an even number of crossings (point B) indicate that the point lies outside the wall boundary. If the number of crossings is odd (point A) then the point lies within the wall boundary. 


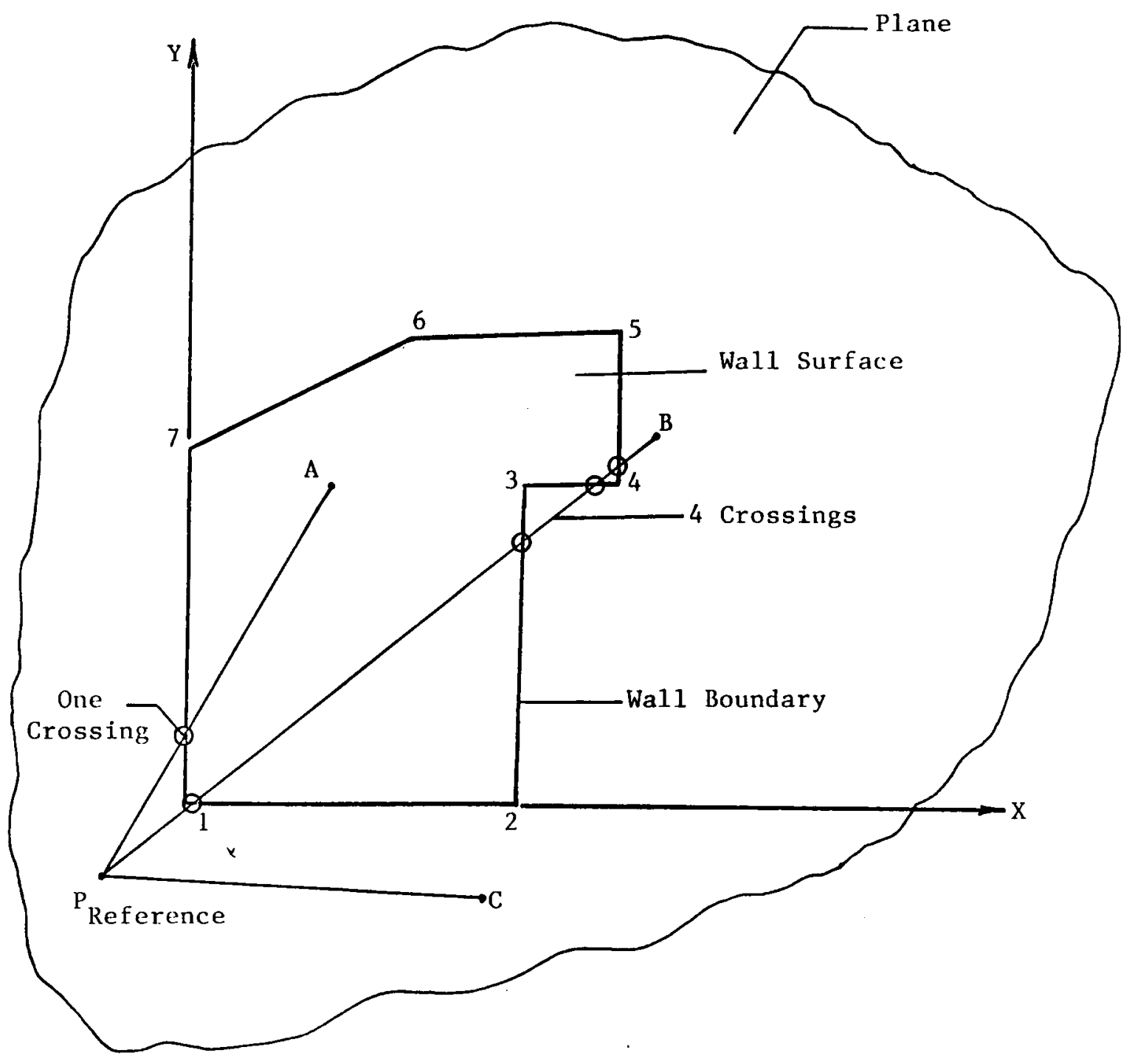

Figure 13. Method devised to determine whether point lies inside or outside wall boundary. 


\subsection{SOUND PRESSURE LEVEL CALCULATIONS}

As indicated in Section 1 the main emphasis in this work was to determine the sound ray paths and then check the sound pressure levels calculated from the ray paths with measured values in a few enclosures. If fairly good agreement was found then the ray tracing equations developed in this work could be used as a basis for further studies. In this section the equations used to calculate the sound pressure levels are discussed. The source was considered to be spherical and consisting of more than one discrete frequency. The latter assumption should be reasonable in most applications since most sources within a factory will not be a pure tone.

Kinsler and Frey ${ }^{11}$ show that for a spherical source of radius $r$ the average energy rate of flow is:

$$
W=4 \pi r^{2} I=\frac{4 \pi r^{2} P^{2}}{2 \rho_{0} C}
$$

where:

$$
\begin{aligned}
& W=\text { Energy flow (watt) } \\
& r=\text { Radius of spherical surface }(\mathrm{m}) \\
& I=\text { Intensity of spherical wave }\left(\text { Watt } / \mathrm{m}^{2}\right) \\
& P=\text { Pressure amplitude of wave }\left(\mathrm{N} / \mathrm{m}^{2}\right) \\
& \rho_{0}=\text { Mean density of medium of wave }\left(\mathrm{kg} / \mathrm{m}^{3}\right) \\
& C=\text { Speed of sound in medium of propagation }(\mathrm{m} / \mathrm{SEC})
\end{aligned}
$$

and since the pressure amplitude of the wave is the real part of the 
spherical wave equation:

$$
P=\frac{A}{r} \operatorname{Cos}(\omega t-k r)
$$

where:

$$
\begin{aligned}
& A=\text { Amplitude constant of wave }\left(N / M^{2}\right) \\
& \omega=\text { Frequency of wave }(h z) \\
& t=\text { Time (seconds) } \\
& k=\text { Wave Number (Radians/M) }
\end{aligned}
$$

or its square may be written as:

$$
P^{2}=\frac{A^{2}}{r^{2}}
$$

Therefore equation (168) may be rewritten as:

$$
W=\frac{2 \pi A^{2}}{\rho_{0} C}
$$

If the sound power level of the source (W) is known then the amplitude constant can be determined by re-writing equation (170) as:

$$
A=\sqrt{\frac{W \rho_{0} C}{2 \pi}}
$$

With the amplitude constant calculated the RMS pressure may be determined by taking the square root of equation (169a) and dividing by the square root of two or

$$
\mathrm{P}_{\mathrm{RMS}}=\frac{\mathrm{A}}{\mathrm{r}}\left[\frac{1}{\sqrt{2}}\right]
$$


The distance $r$ is the path length of the rays and is determined from the ray segment end points using the pythagorean theorem $r(N)=\left[[X(N)-X(N-1)]^{2}+[Y(N)-Y(N-1)]^{2}+[Z(N)-Z(N-1)]^{2}\right]^{\frac{1}{2}}$

where:

$r(N):$ Path length (m)

$X(N), Y(N), Z(N)$ : Coordinates of $N$ th point on ray path (m)

$\mathrm{X}(\mathrm{N}-1), \mathrm{Y}(\mathrm{N}-1), \mathrm{Z}(\mathrm{N}-1):$ Coordinates of point immediately preceding Nth point (m)

Equation (171) determines the RMS sound pressure along a ray path without considering intensity losses due to reflections. When sound impinges on a surface a certain amount of its energy is absorbed. The fraction of incident energy is given by (1-Absorption Coefficient) of that surface.

Thus :

$$
P_{\mathrm{RMS}}=\mathrm{P}_{\mathrm{RMS}}(1-\alpha)^{\frac{1}{2}}
$$

where:

\section{$\alpha$ : Absorption Coefficient of surface}

This relationship is applied successively at each reflection point along each ray path. The energies arriving at a location along eacy ray path are then sumed. The sound pressure level is then calculated by:

$$
\text { SPL }=10 \text { LOG }\left[\frac{P_{\text {RMSt }}}{P_{\text {REF }}}\right]^{2}
$$


where:

$$
\begin{aligned}
& \mathrm{P}_{\mathrm{RMS}_{\mathrm{t}}}=\text { Total RMS sound pressure }\left(\mathrm{N} / \mathrm{m}^{2}\right) \\
& \mathrm{P}_{\mathrm{REF}}=\text { Reference sound pressure }\left(2 \times 10^{-5} \frac{\mathrm{N}}{\mathrm{m}^{2}}\right)
\end{aligned}
$$

The preceding procedure may be applied to the calculation of overall sound pressure levels of octave band sound pressure levels, or of an A-weighted sound pressure level, as desired.

To calculate overall sound pressure levels the octave band acoustic power of the source must be used along with the octave band absorption characteristics of each surface. The above procedure is applied to each frequency using the appropriate power level and absorption coefficient. When the sound pressure level for each frequency has been determined the corresponding total sound pressure level is determined by adding the square of the RMS sound pressure at each frequency.

$$
\left(\mathrm{P}_{\mathrm{RMS}}\right)^{2}=\left(\mathrm{P}_{\mathrm{RMS}_{1}}\right)^{2}+\left(\mathrm{P}_{\mathrm{RMS}_{2}}\right)^{2}+\ldots+\left(\mathrm{P}_{\mathrm{RMS}_{8}}\right)^{2}
$$

Equation (174) is then used to determine the total sound pressure level in $\mathrm{dB}$.

The $\mathrm{dBA}$ calculation is performed in the same manner except that after the sound pressure levels in $\mathrm{dB}$ are determined at each frequency they must be weighted (adjusted) at each frequency before equations (174) and (175) are used. This procedure is illustrated in Table 1. 
TABLE 1

Illustration of Conversion from $\mathrm{dB}$ Overall to dBA Overall

\begin{tabular}{cccc}
\hline $\begin{array}{c}\text { FREQUENCY } \\
\text { HZ }\end{array}$ & $\begin{array}{c}\text { SOUND PRESSURE } \\
\text { LEVEL }\end{array}$ & $\begin{array}{c}\text { A-WEIGHTING } \\
\text { RELATIVE } \\
\text { RESPONSE }\end{array}$ & $\begin{array}{c}\text { A-WEIGHTED } \\
\text { SOUND PRESSURE } \\
\text { LEVEL }\end{array}$ \\
\hline 63 & 75.6 & -26.2 & 49.4 \\
125 & 74.6 & -16.1 & 58.5 \\
250 & 74.6 & -8.6 & 66.0 \\
500 & 74.3 & -3.2 & 71.1 \\
1000 & 74.2 & 0.0 & 74.2 \\
2000 & 74.3 & +1.2 & 75.5 \\
4000 & 72.3 & +1.0 & 73.3 \\
8000 & 71.4 & -1.1 & 70.3 \\
\hline
\end{tabular}

OVERALL

SOUND PRESSURE

83.1

80.5

LEVEL 
The results in column 2 are the $d B$ calculations at each frequency shown in column 1. The A-weighting relative response, e.g., the adjustment to be made of the values in column 2 at each frequency, are shown in column 3. The last column is the resulting A-weighted frequency spectra determined by adding columns 2 and 3 . The overall sound pressure level for the unweighted and the A-weighted spectra is the quantity at the bottom of columns 2 and 4 . 


\subsection{APPLICATION OF PROGRAM}

\subsection{Ray Tracing Plots}

One of the several forms of the program output available is the plot of the calculated ray paths. These plots verify the input to the program and also allow one to see the ray paths and their reflection locations. With this knowledge it is possible to determine the best areas to add absorption material to reduce the reverberant contribution of the total sound pressure level at a given location. The reflection locations would not help in the case where the receiver is in the near field of the source and the sound pressure level is a function of the radiated sound power level of the source.

Two examples will be presented to demonstrate the use of the ray paths. The first example is a room $98.4 \mathrm{~m} . \mathrm{X} 65.6 \mathrm{~m} . \mathrm{X} 36 \mathrm{~m}$. To illustrate more clearly the ray paths, the program was altered slightly to give three separate views of the room with the direct ray and all single reflection cases in the first view, all two reflection rays in the second view and all three reflection rays in the third view. These ray plots are shown in Figures 14, 15 and 16, respectively. A plan and elevation view is provided in each Figure showing reflections from all the surfaces. These Figures show no specific areas where absorption material would aid in reducing the reverberant part of the sound pressure level at the indicated receiver position. 

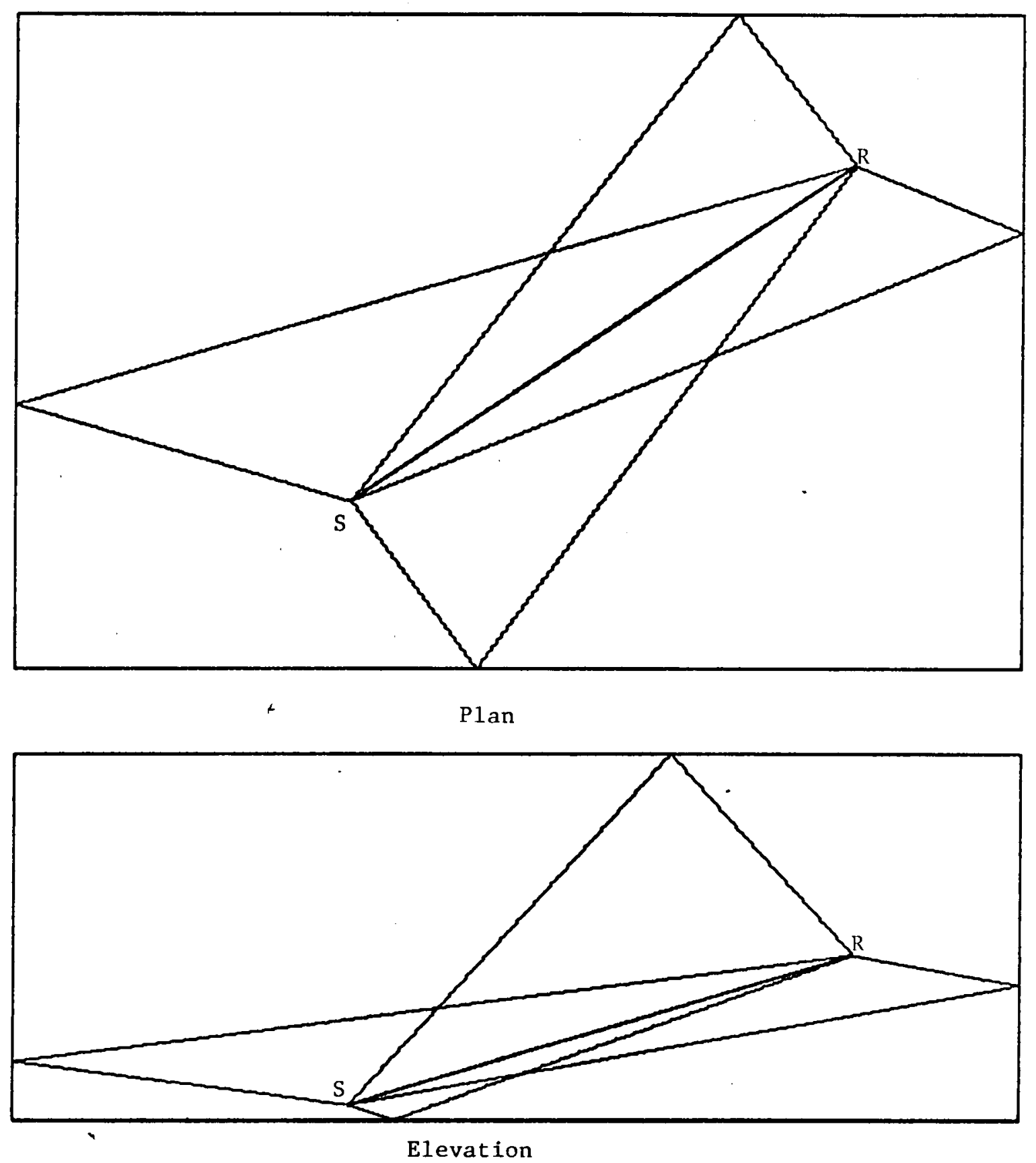

Figure 14. Direct ray and all single reflection ray paths, Randolph classroom. 

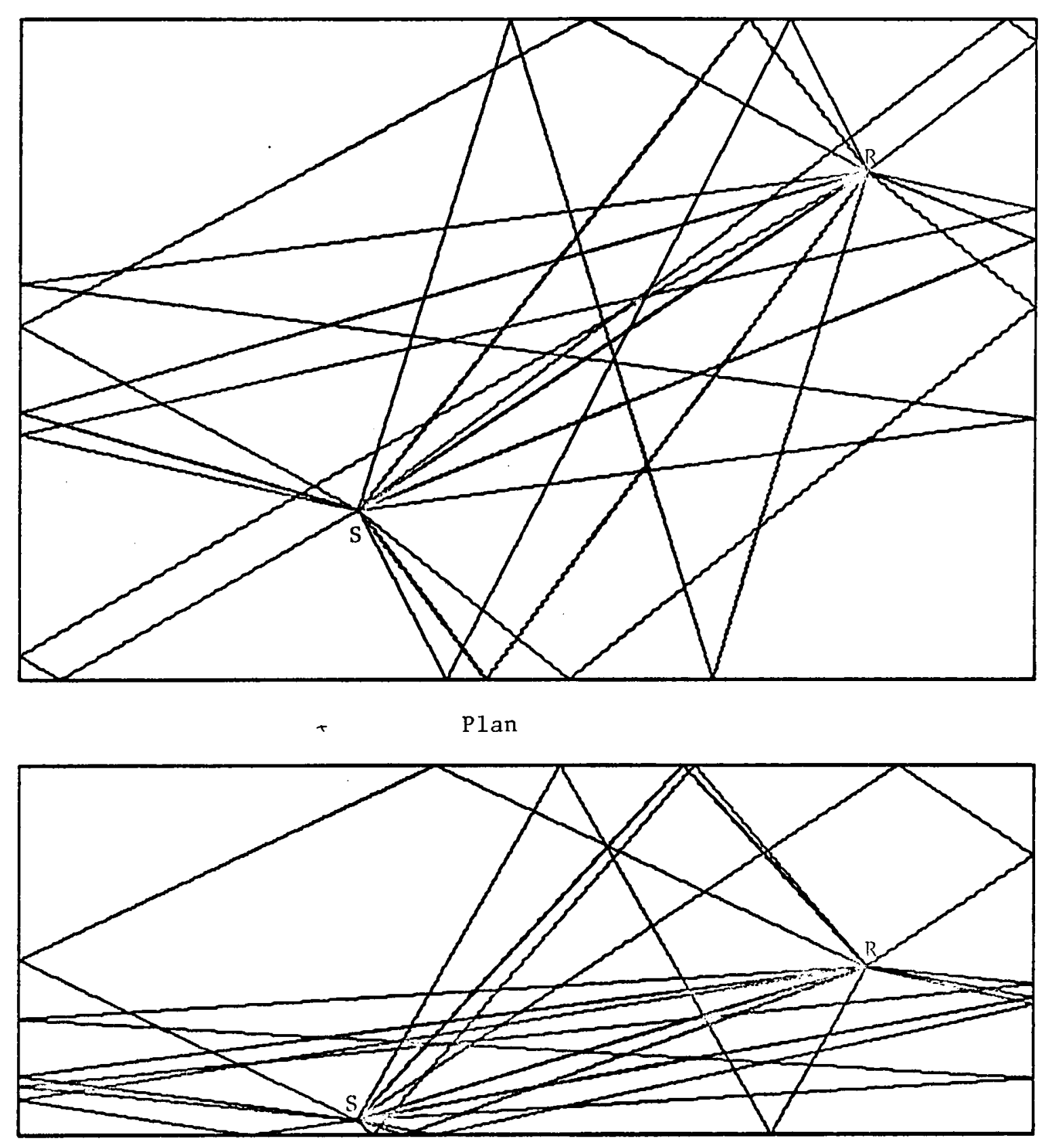

Elevation

Figure 15. All valid sound rays undergoing two reflections, Randolph classroom. 


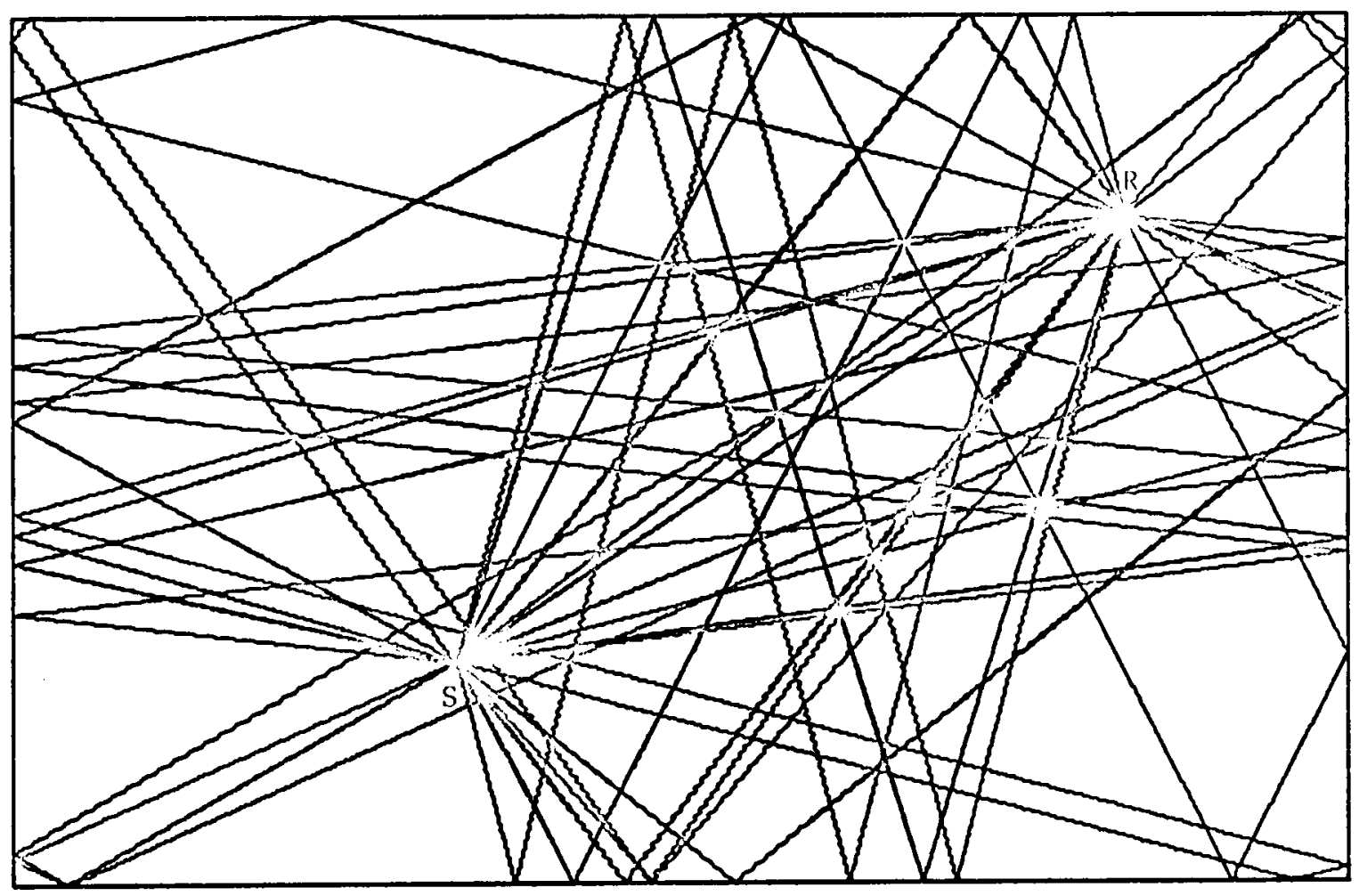

Plan

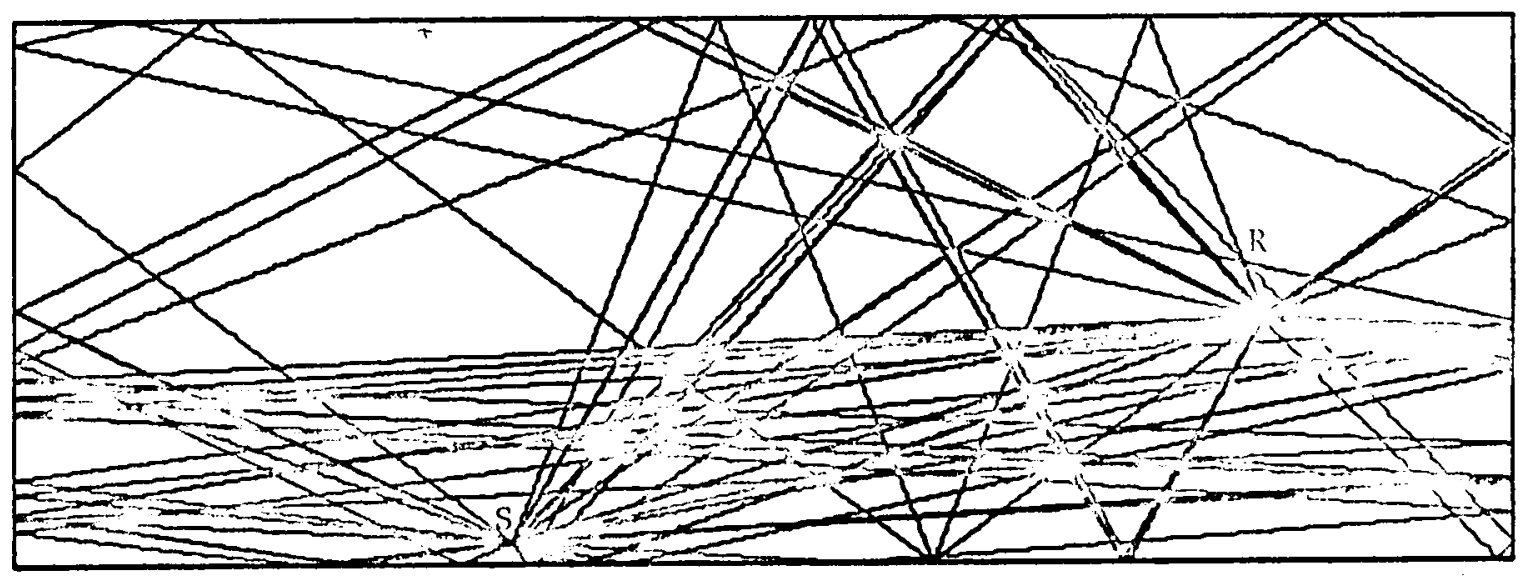

Elevation

Figure 16. All valid sound rays undergoing three reflections, Randolph classroom. 
The second example shown is floor plan from an existing industrial plant. Three plan views were drawn with different source and receiver locations. Instead of drawing separate elevation and plan views $X^{\prime}$ 's and $0^{\prime} s$ were used to indicate reflections from the ceiling and floor respectively.

Figures 17,18 and 19 show the resulting ray traces. The ray tracing plot shown in Figure 17 reveals that absorption material would be effective on areas $A$ and $B$. The octave band sound pressure level predictions would indicate the necessary frequency properties of the absorption to be used. This figure shows that the addition of absorption material at any other location would be an impractical and relatively ineffective solution to a noise problem at this receiver location. In Figure 18 the source and receiver have been moved closer together. Several reflections still occur at locations $A$ and $B$ but additional surfaces are involved as shown in section C and D.

At this point an observer might look at Figure 18 and think that a more diffuse sound field exists there than in Figure 17 . It is important to realize that the ray traces only show those rays from the source that reach the receiver in three reflections (except in the case of slanted surfaces where a maximum of two reflections are calculated). Since rays shown are a function of only the one given source at a given receiver location the ray traces do not tell us whether a diffuse sound field exists. Also, since the ray plots 


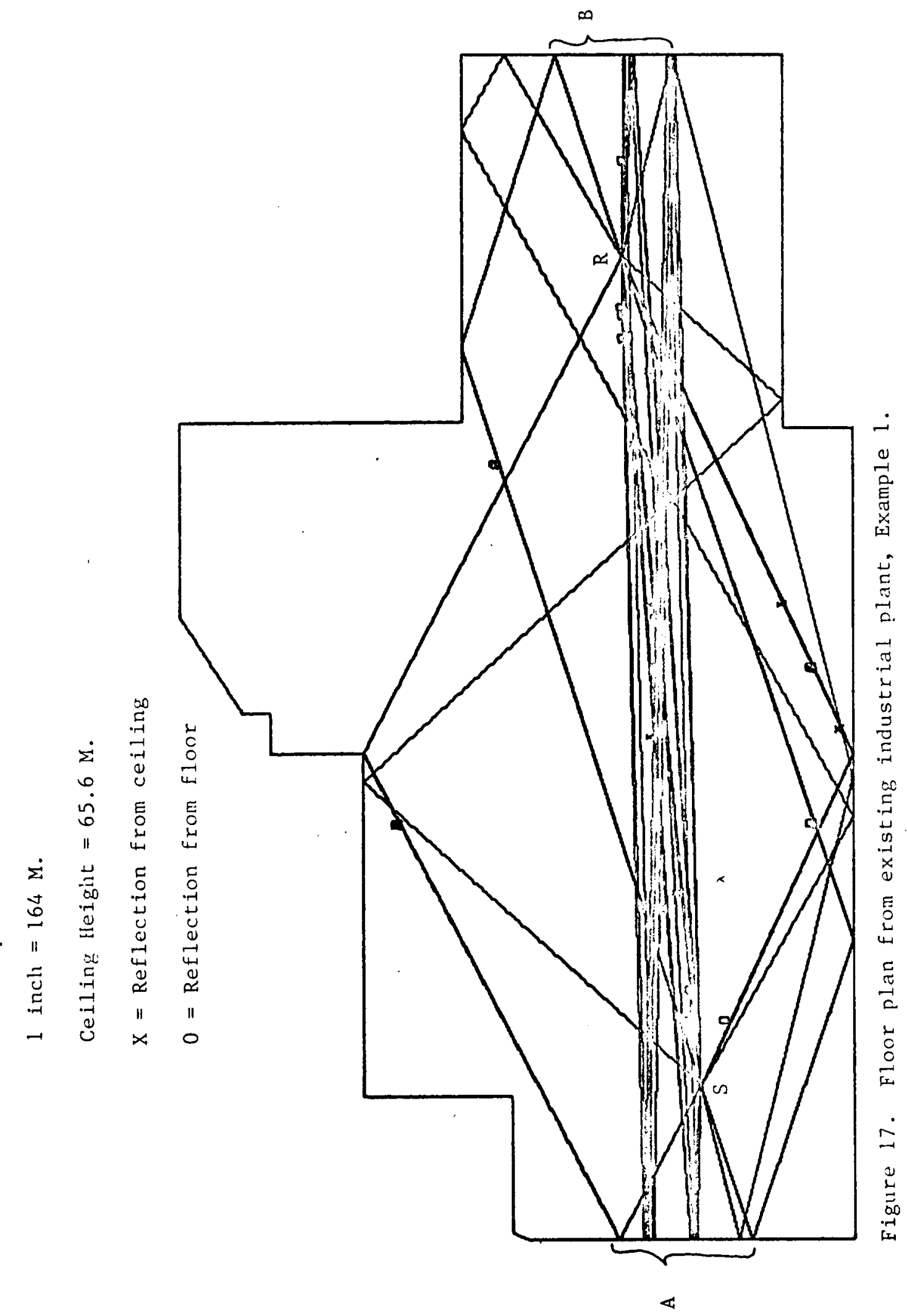




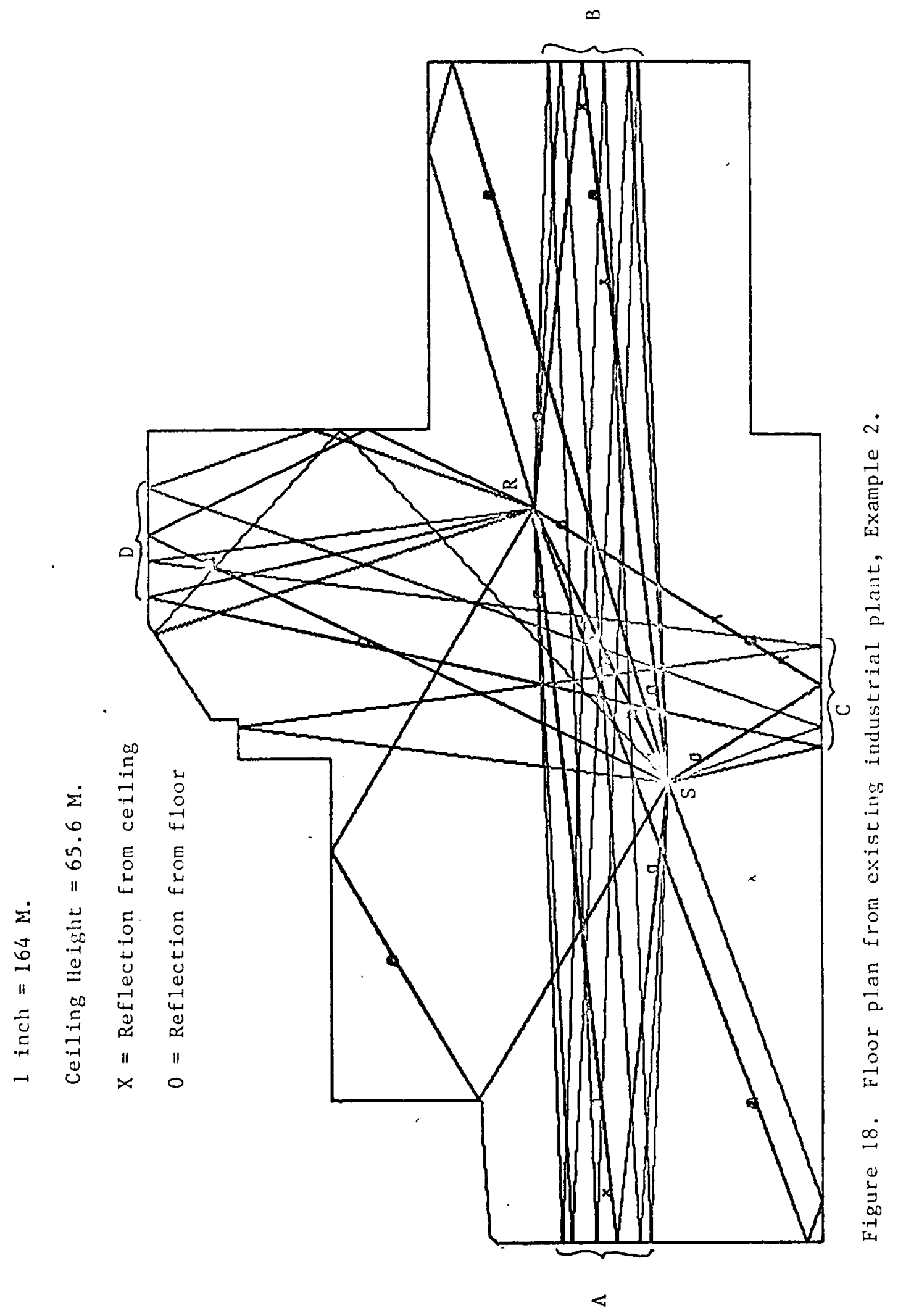




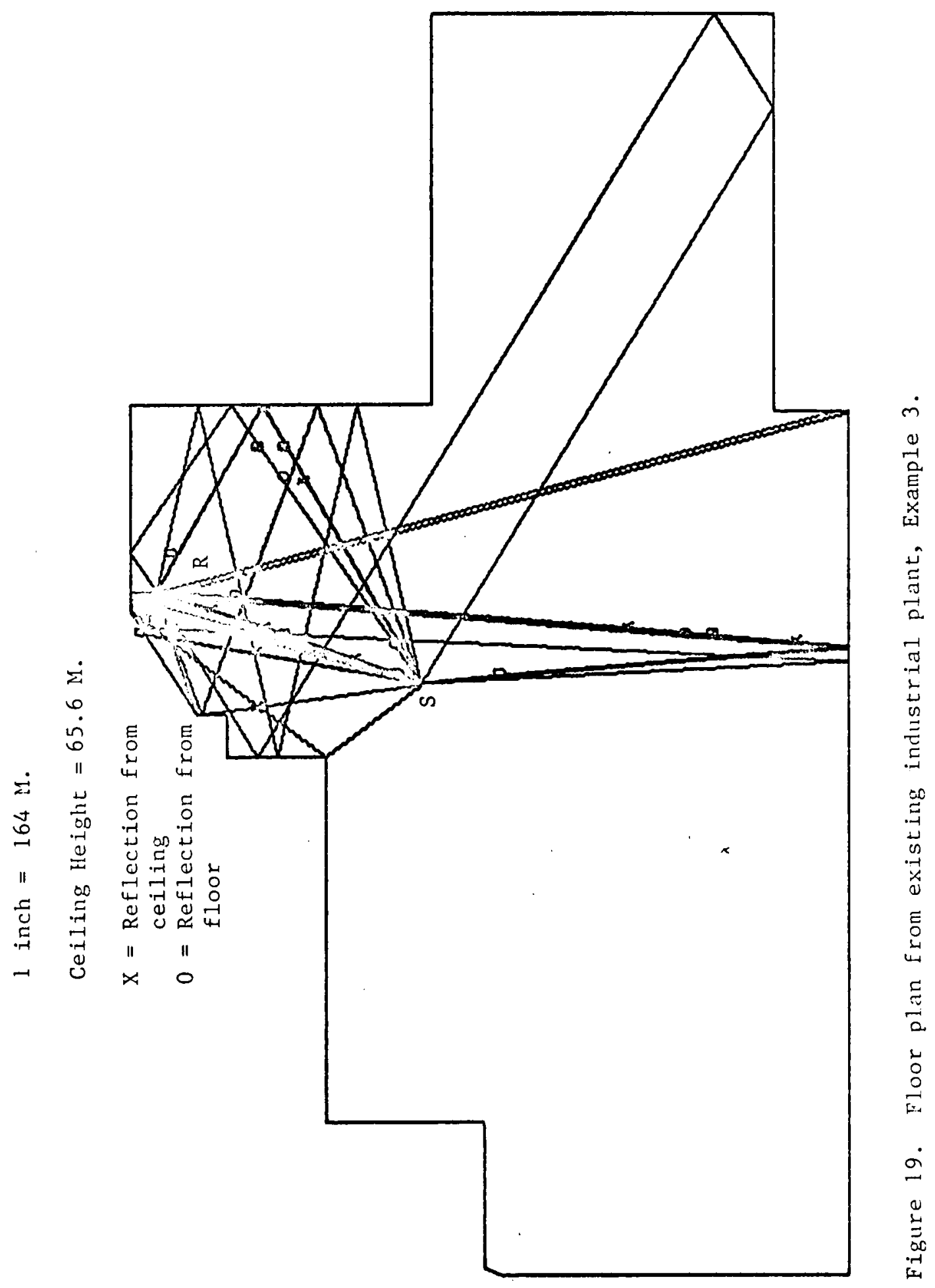


Indicate areas where sound absorption material should be added the reader should realize that this will only reduce the noise level at that one particular receiver location. High sound pressure levels at other locations may or may not be reduced.

The program's ability to handle slanted surfaces is shown in Figure 19. This ability allows the program to handle surfaces as indicated in the figure and also factory spaces with inclined roofs. With additional development of the program this ability will allow for slanted barriers within a factory space. Another point of interest concerning Figure 19 is that the primary reflection surfaces are those in that portion of the factory space where the slanted surface is located. This can be compared with Figure 17 where no reflections occur in that same area and Figure 18 which has reflection locations in common with Figures 17 and 19.

6.2 Comparison of Predicted and Measured Sound Pressure Levels The program's ability to determine acoustic ray paths in enclosed spaces has now been shown. In this section actual measured sound pressure levels are compared with sound pressure levels which were calculated using the ray path lengths. The results of these comparisons will help determine whether the use of this ray tracing technique to predict sound pressure levels should be investigated further. 
Sound pressure leve1 measurements were made in two different rooms and used to check the accuracy of the predicted sound pressure levels. An ILG sound source, whose octave band sound power is shown in Table 2 was used in each experiment. Measurements were taken at several locations within the room. The measured sound pressure levels were then compared with the predicted sound pressure levels determined with the computer programs.

The first room was the same room used in the initial ray tracing example (Figures 14,15,16) with dimensions of $98.4 \mathrm{~m} \times 65.6 \mathrm{~m} \times$ $36 \mathrm{~m}$ The room was a classroom with painted concrete walls, an asphalt tiled floor and a ceiling of acoustic tile. The absorption characteristics of these surfaces are shown in Table 3. Measured and calculated sound pressure levels were determined at thirteen meter intervals throughout the room. The resulting measured sound pressure levels in $\mathrm{dBA}$ are shown in Figure 20. The calculated sound pressure levels in dBA are shown in Figure 21. The difference between predicted and measured levels is less than $3 \mathrm{dBA}$ at a11 locations.

The agreement between the predicted and measured sound pressure levels can also be seen in Figure 22, which is a plot of the predicted and measured sound pressure levels at the circled positions one through seven shown in Figures 20 and 21 . The results show good agreement between predicted and measured sound pressure levels in terms of the 


\section{TABLE 2}

Calibration of ILG Reference

Sound Source*

Octave Center

Frequency $\mathrm{Hz}$
Sound Power Level dB re $10^{-12}$ watt

*Manufacturer's data from ILG Industries, Inc., 2826 North Pulaski Road, Chicago, I1linois 60641. 
TABLE 3

\author{
Octave Band Absorption Characteristics \\ of Surfaces in Randolph \\ Class Room*
}

\title{
MATERIAL
}

Frequency

$\begin{array}{rccc}63 & .04 & .01 & .4 \\ 125 & .04 & .01 & .47 \\ 250 & .03 & .02 & .65 \\ 500 & .05 & .02 & .75 \\ 1000 & .11 & .02 & .84 \\ 2000 & .07 & .02 & .83 \\ 4000 & .02 & .02 & .81 \\ 8000 & .02 & .02 & .8\end{array}$

*Taken from Acoustical Designing in Architecture by $V .0$. Knudsen and C. M. Harris, John Wiley and Sons, New York 1950. 


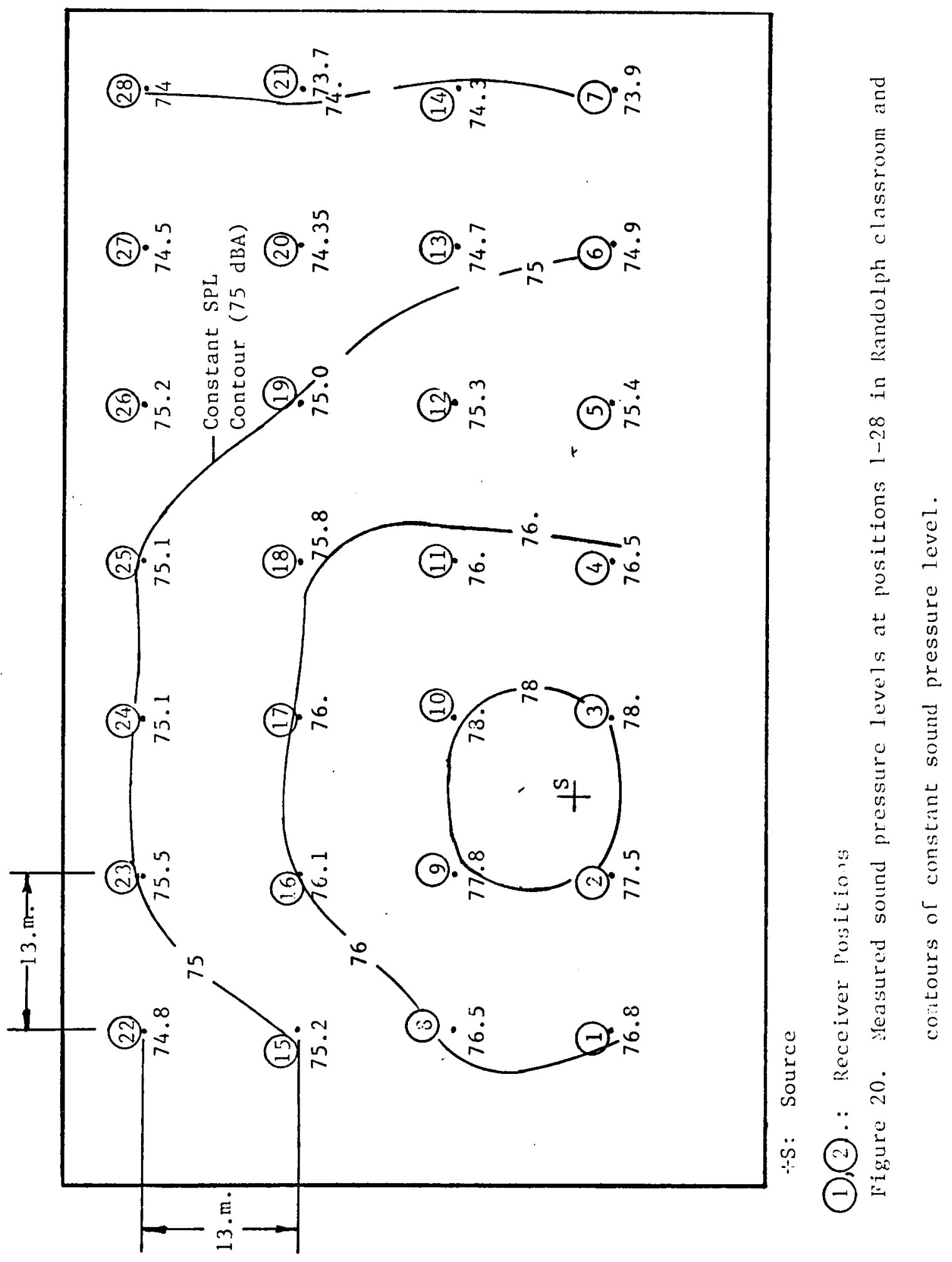




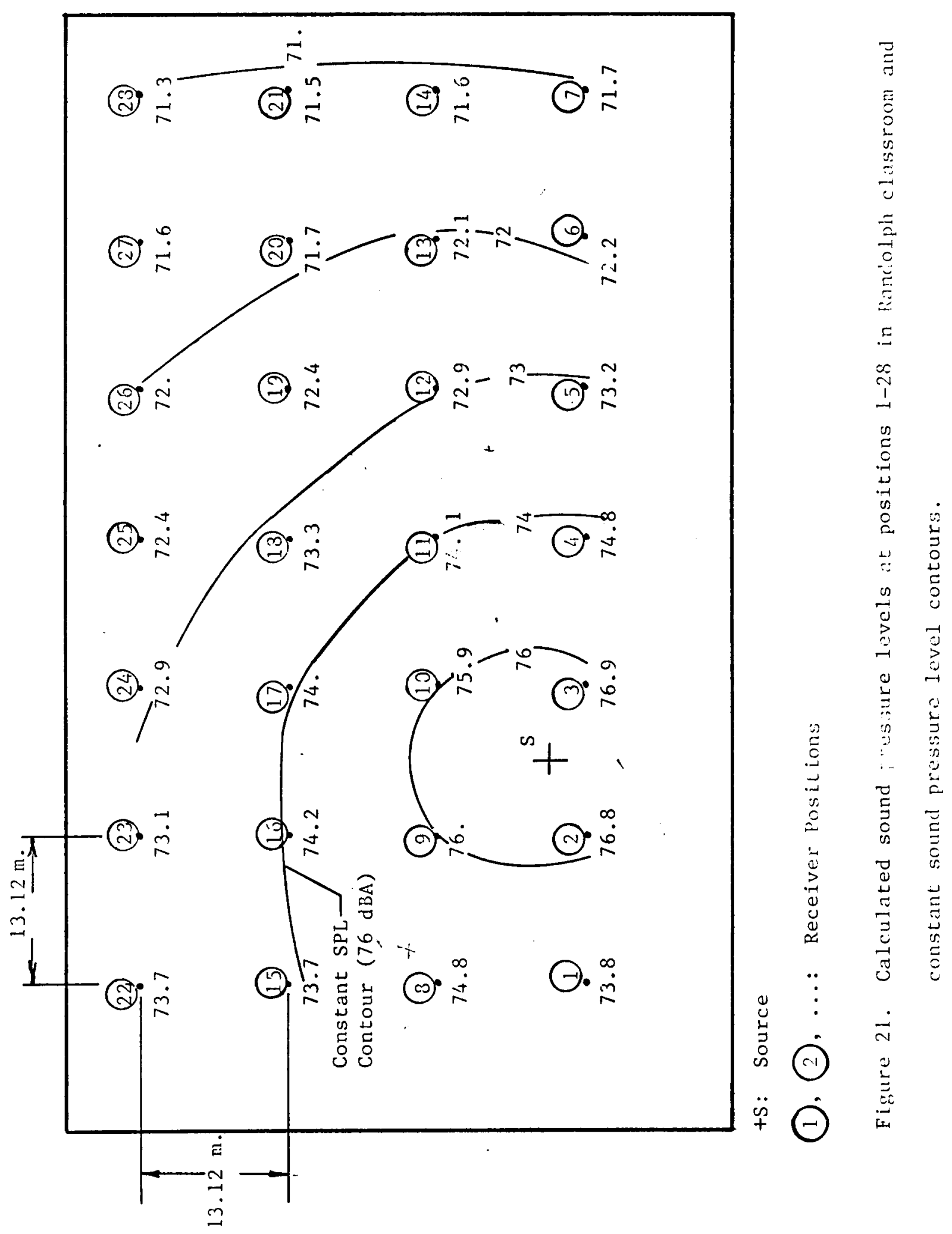




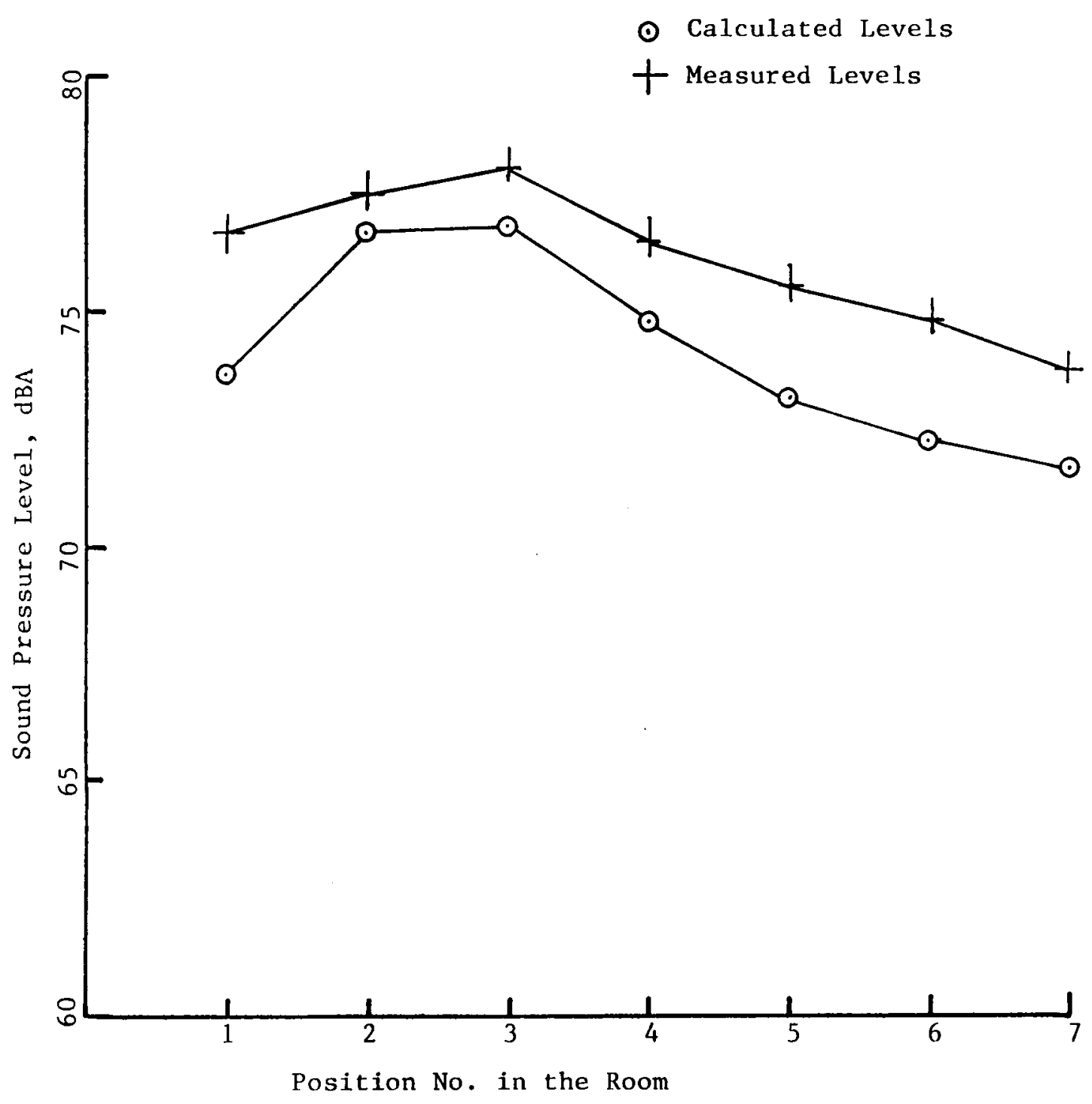

Figure 22. Comparison of measured and calculated dBA sound pressure levels in Randolph Classroom at positions $1,2,3,4, \ldots, 7$, best agreement. 
curve shape and the actual sound levels. However, the predicted sound pressure levels are always lower than the measured sound pressure levels.

The least agreement between measured and predicted $\mathrm{dBA}$ sound pressure levels occurred at positions twenty-one through twenty-eight, as shown in Figure 23. However, the differences between predicted and measured sound pressure levels were less than $3 \mathrm{dBA}$ as mentioned previously and the curve shapes were quite similar.

To find the reason why some sound pressure level predictions in $\mathrm{dBA}$ were better than others, plots were made of the sound pressure levels as predicted in each octave band. This was done at points 2 and 26. The results are shown in Figures 24 and 25, respectively. The results at position 2 indicate decreased prediction accuracy at frequencies of $500 \mathrm{~Hz}$ or less. Since the overall levels plotted in Figure 22 were on the $d B A$ weighted network the low frequency sound pressure levels contributed less to the resultant dBA value.

The frequency plot in Figure 25 shows fair to poor agreement between predicted and measured sound pressure levels at middle and upper frequencies as well as at the lower frequencies. For this reason the dBA results shown in Figure 23 were less accurate.

The background noise was not a contributing factor to the disagreement between predicted and measured SPL, because the difference between the background measurements and the experimental measurements were more than $10 \mathrm{~dB}$ at all frequencies. 


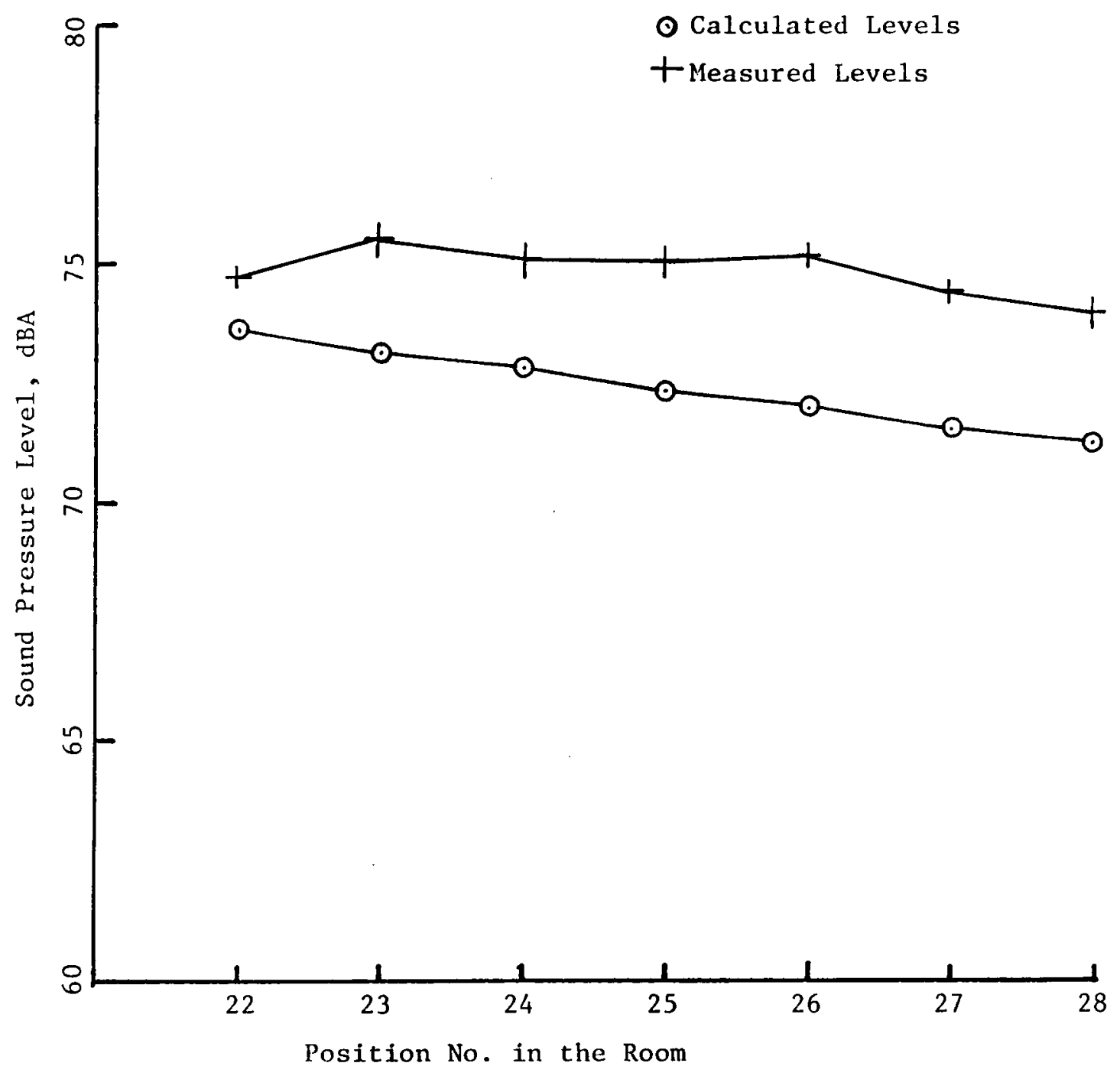

Figure 23. Comparison of measured and calculated dBA sound pressure levels in Randolph Classroom 22, 23, $24, \ldots, 28$, worst agreement. 


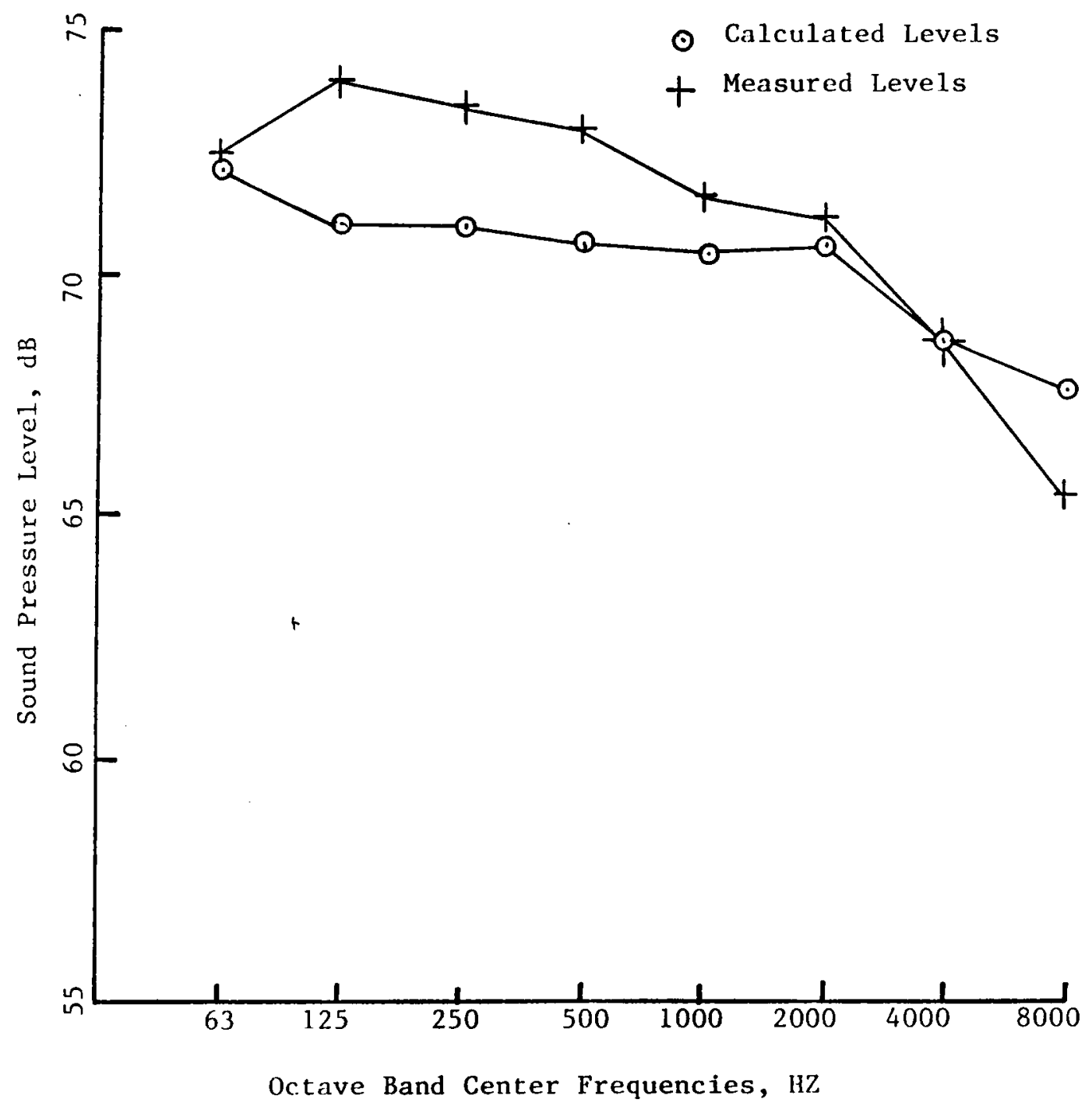

Figure 24. Comparison of measured and calculated octave band sound pressure levels at position 2 in Randolph Classroom 


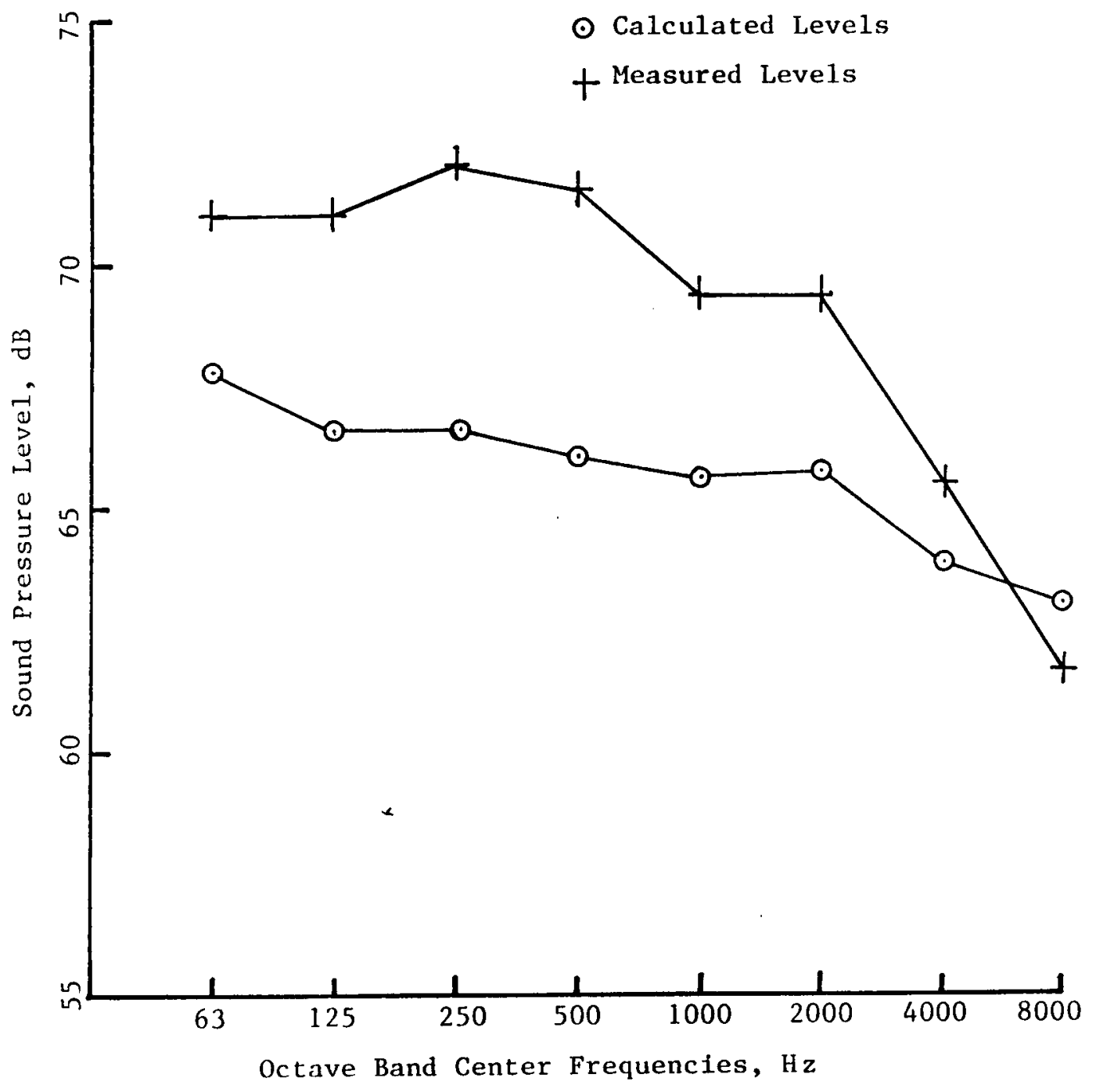

Figure 25. Comparison of measured and calculated octave band sound pressure levels at position 26 in Randolph Classroom 
Rather than discuss other possible reasons for errors at this point a comparison between predicted and measured sound pressure levels in a hallway will be shown and a general discussion of sources of error will follow.

A long narrow hallway $2 \mathrm{~m} \times 2.4 \mathrm{~m} \times 30 \mathrm{~m}$ was the second site used to check the predictive capabilities of the program. Figure 26 shows the room and the locations where measured and calculated sound pressure levels were determined. The walls were painted concrete, the floor was asphalt tile and the ceiling was acoustic tile. (Table 3).

The results of the measured and predicted sound pressure levels in $\mathrm{dBA}$ are shown in Figure 27. These results show that the predicted levels are within $4 \mathrm{dBA}$ of those measured at the most remote position down the hall. The measured value at position 7 is likely an error in the meter reading. If a continuous curve is drawn through the other points this poirft will be excluded.

Overall sound pressure level predictions shown in Figure 28 indicate less accuracy than the dBA predictions shown in Figure 27. A look at the octave band spectra showed least accuracy at the lower frequencies. These would show up in the overall sound pressure level predictions but to a lesser extent in the dBA predictions as illustrated in the previous example. The two octave band spectra are shown in Figures 29 and 30 which were determined at points 2 and 13 


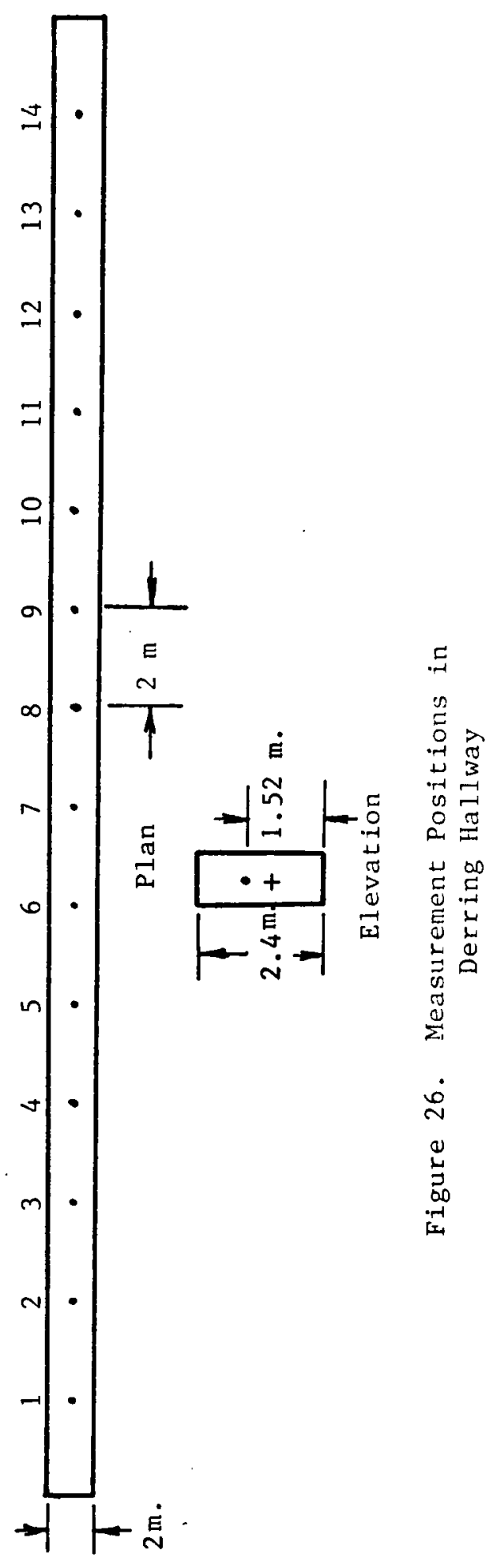




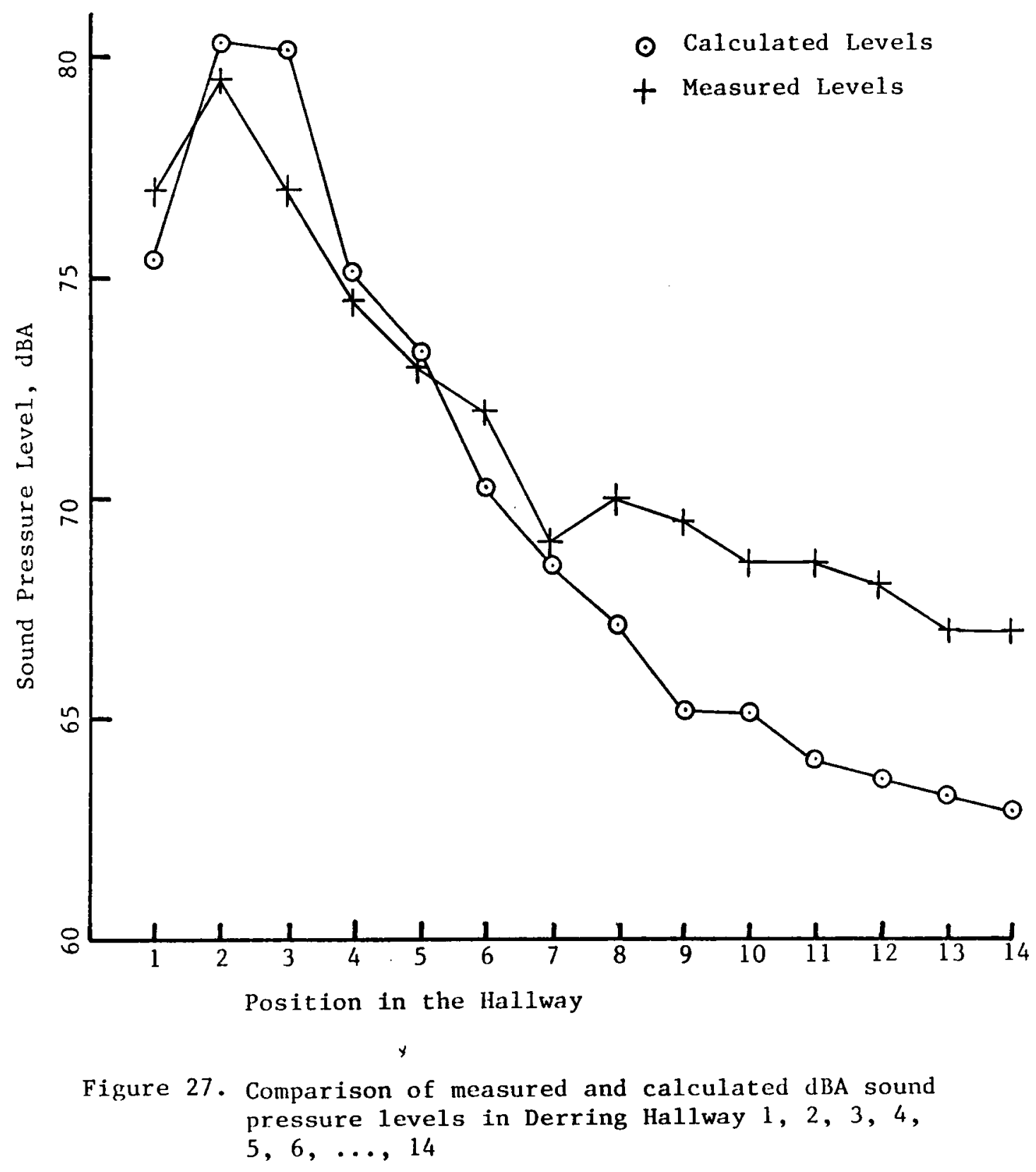




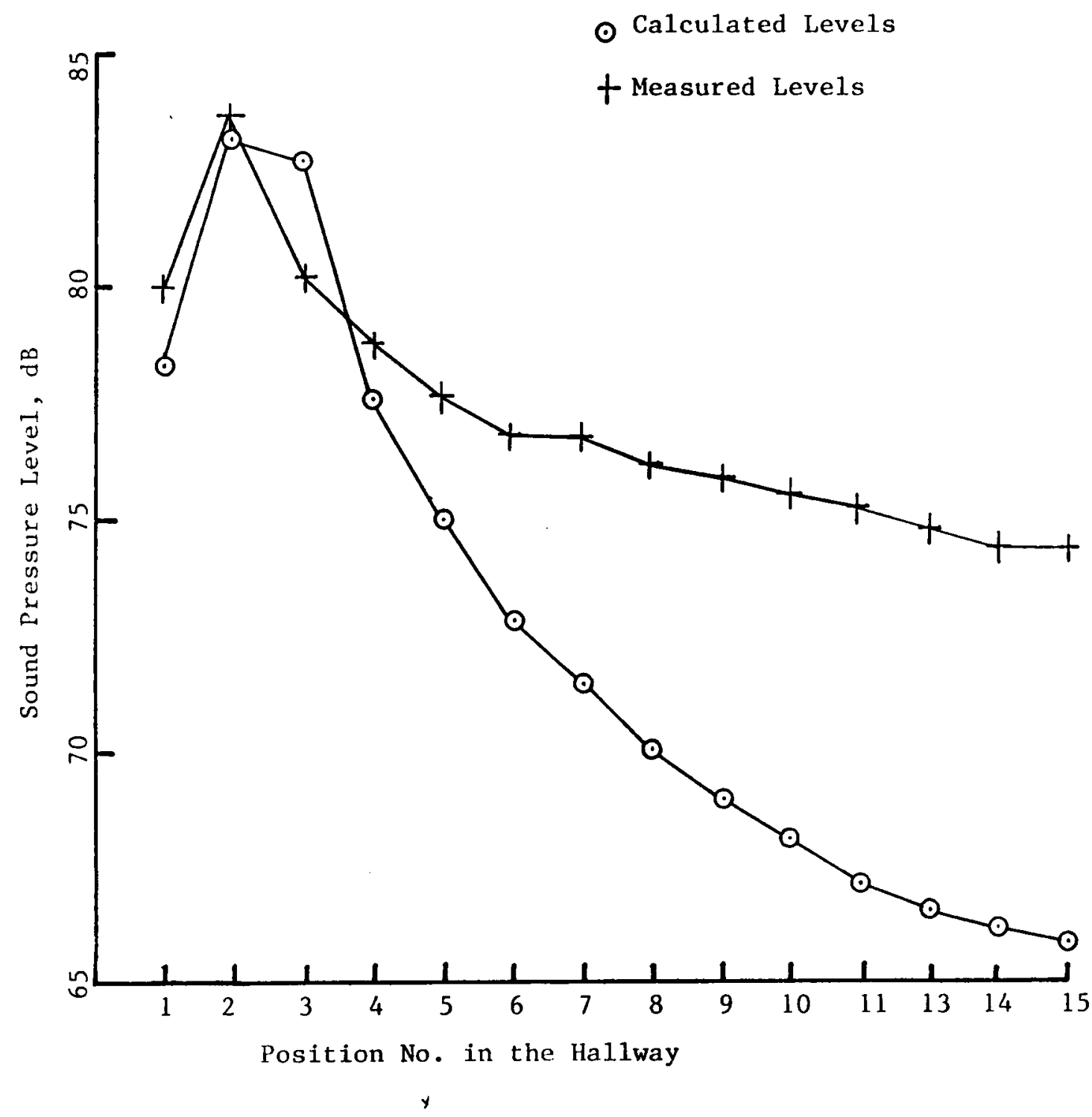

Figure 28. Comparison of measured and calculated overall sound pressure levels in Derring Hallway at positions $1,2,3, \ldots, 14$ 


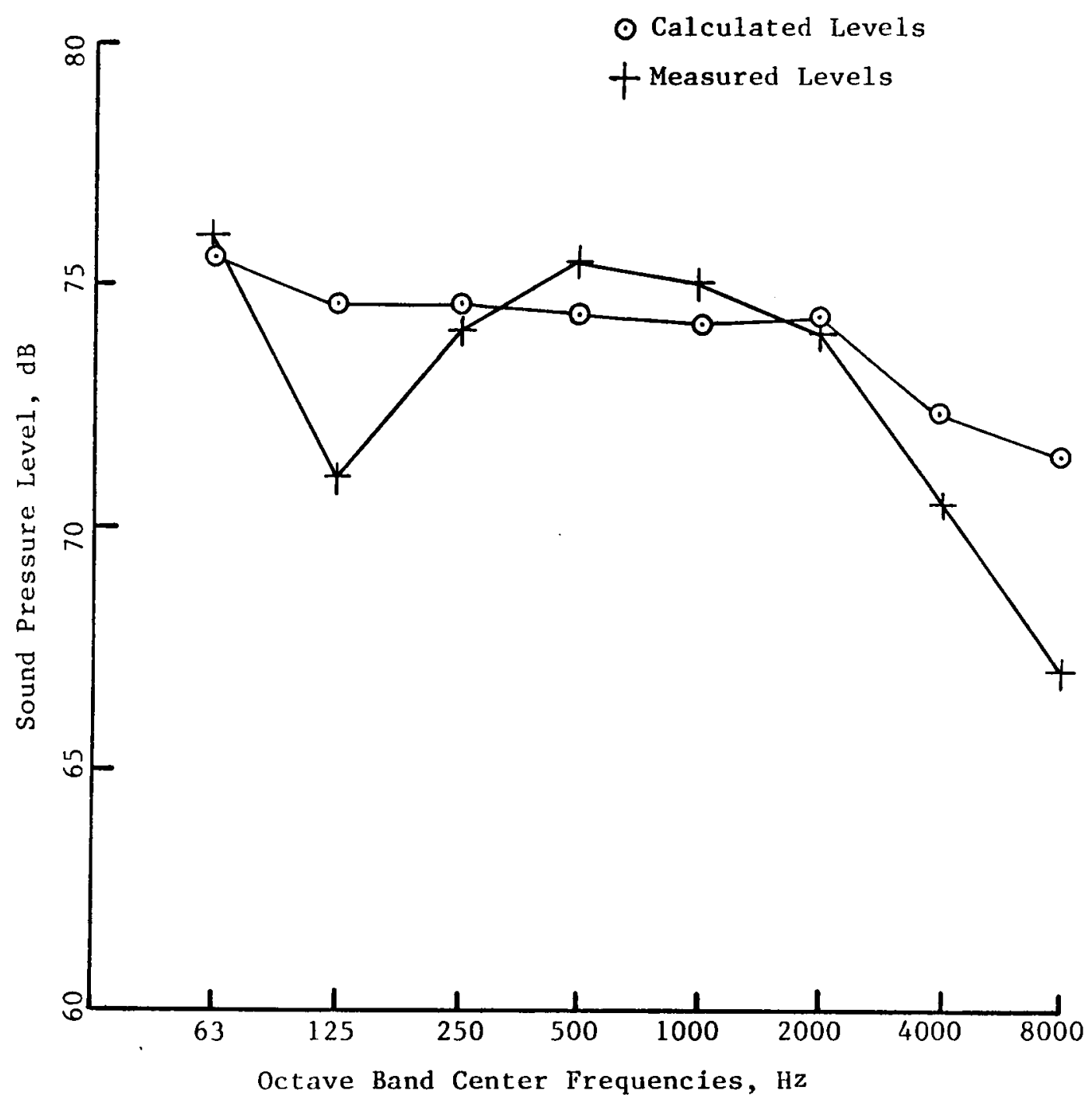

Figure 29. Comparison of measured and calculated octave band sound pressure levels at position 2 in Derring Ha1lway, best agreement. 


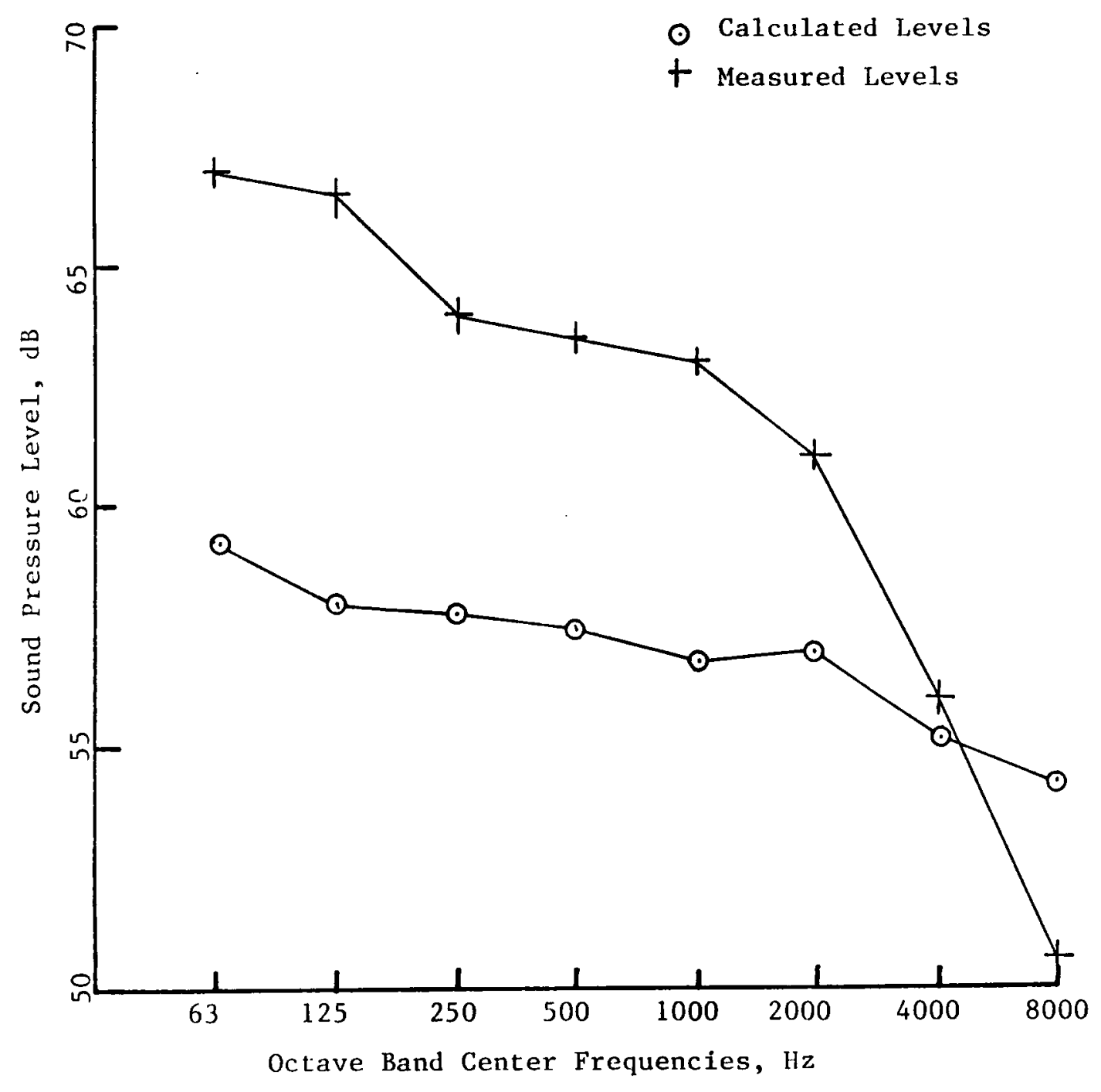

Figure 30. Comparison of measured and calculated octave band sound pressure levels at position 13 in Derring Hallway, worst agreement. 
respectively. Data taken at point 2 shows excellent agreement at all but the $125 \mathrm{~Hz}$ frequency. Farther down the hall away from the source (point 13) the octave band spectrum reveals a 6-7 dB disagreement at most frequencies and the maximum at frequencies below $250 \mathrm{~Hz}$. With these results in mind one can understand more easily the results shown earlier in Figures 27 and 28. It is evident that the further the receiver is from the source the less accurate the predictions. The dBA predictions are in better agreement further down the hall than the overa11 sound pressure levels.

The results in the hallway experiment and the classroom experiment indicate the program predicts sound pressure levels better at higher frequencies than lower frequencies. Possible sources of error in the low frequency spectrum may lie in the fact that the geometric acoustics theory is only valid as a predictive tool when the wavelengths of the sound are much less than the dimensions of the room. The lowest frequency considered in this study was $63 \mathrm{~Hz}$. The wavelength at this frequency is about 5.5 meters. The smallest dimension in Derring Hall was 7 meters which may account for the sound pressure level inaccuracies at low frequencies. Bolt, Doak, and Westervelt ${ }^{6}$, as discussed in Section 2.2, indicate that for fairly large rooms, like auditoriums, results using geometric acoustics are valid for frequencies above $100 \mathrm{~Hz}$. 
The fact that the low frequency sound pressure levels are predicted with less accuracy is not as serious a problem as it first appears because the primary interest is dBA. This depends upon the middle to upper frequency predictive capabilities of the program.

In the case of a very long and very narrow room (hallway) the predicted sound pressure levels may be less accurate at great distances because inclusion of sound rays with no more than three reflections does not adequately describe the behavior of the sound in this duct-like environment. This problem needs further considerations. However, it is believed that a small proportion of factories will possess rooms where one dimension is so much greater than the other two. Thus, this might not prove to be a troublesome limitation. 


\subsection{CONCLUSIONS}

The results of the theoretical and experimental investigations allow some conclusions to be drawn:

1. The program can handle irregular rooms including room boundaries with slanted surfaces.

2. The ray tracing plots can indicate areas where absorption material may be added to reduce sound levels at a given receiver location.

3. The sound pressure level predictive capabilities of the program are promising and justify further work using the methods determined in this work.

Recommendations for the improvement of the program include investigating different intensity calculations not based on a point sound source. Directivity of the source and diffraction effects in irregular rooms need to be incorporated into the program. The program should also be extended to handle barriers within a room. Investigations into the effects of air absorption should also be considered. 


\subsection{BIBLIOGRAPHY}

1. Public Law 91-596, "Occupational Safety and Health Act of 1970", 91st Congress, S.2193, Dec. 29, 1970.

2. Beranek, L. L., "Sound in Large Rooms", Chapter 9 of Noise and Vibration Control, edited by L. L. Beranek, McGraw-Hi11, New York, 1971.

3. Lester, D. H., Jr., Sound Pressure Level Predictions in Large Rooms, M. S. Thesis, V.P.I. \& S.U., May 1973.

4. Gibbs, B. M. and Jones, D. K., "A Simple Image Method for $V$ Calculating the Distribution of Sound Pressure Levels Within an Enclosure", Acoustica, Vol. 26, 1972, pp. 24-30.

5. Officer, C. B., Introduction to Theory of Sound Transmission, McGraw-Hill, New York, 1958.

'6. Bolt, R. H.; Doak, P. E.; Westervelt, P. J., "Pulse Statistics Analysis of Room Acoustics", Journal of Acoustical Society of America, Vol. 22, 1950, pp. 328-340.

7. Krokstad, A.; Strom, S.; Sorsdal, S., "Calculating the Acoustical Room Response by the Use of a Ray Tracing Technique", Journal of Sound and Vibration, Vol. 8, 1968, pp. 118-125.

8. Stenseng, N., The Influence of Diffusors on Sound Absorption Measurements in a Sma11 Reverberation Chamber, Thesis, University of Oslo, Institute of Physics, 1965.

9. Lowe, A. W., "The Effectiveness of Barriers Under Extended Ceilings", Inter Noise 72 Precedings, 1972, pp. 89-94.

"10. Barron, M., "Growth and Decay of Sound Intensity in Rooms According to Some Formulae of Geometric Acoustics Theory", Journal of Sound and Vibration, Vol. 27, 1973, pp. 183-196.

11. Kinsler, L. E.; Frey, A. R., "Spherical Acoustic Waves", Chapter 7 of Fundamentals of Acoustics, John Wiley \& Sons, Inc., New York, 1962 . 
APPENDIX A

USERS GUIDE AND PROGRAM DOCUMENTATION 
Users Manua1

The necessary steps to use the program in its current form are as follows:

The user must first assemble the following information:

1. Drawings (floor plan and elevation) of factory space;

2. Composition of all interior surfaces of factory space;

3. Location of all sound sources;

4. Octave band (if possible) or overall sound power levels of each source.

With the information from item (1) the plant should be laid out on grid paper as shown in Figure A-1. It is necessary to assure that all of the factory is positioned in the first quadrant as shown in the example. The sources from item (3) should also be located on the grid as shown $\left(s_{1}, s_{2}, s_{3}\right)$.

The octave band absorption coefficients must then be found, either from vendor data or other sources.

The necessary data can now be put into the program on cards, or as a data set. To illustrate the procedure the factory space in Figure A-1 will serve as an example. The necessary data to be put into the program is shown in Figure $\mathrm{A}-2^{*}$ and should be referred to as the procedure is explained. It is imperative that the input be placed in the indicated columns in Figure A-2.

The first data card tells the program whether a ray tracing plot is desired in the output. If a plot is desired a one is * May be found in packet at end of thesis. 


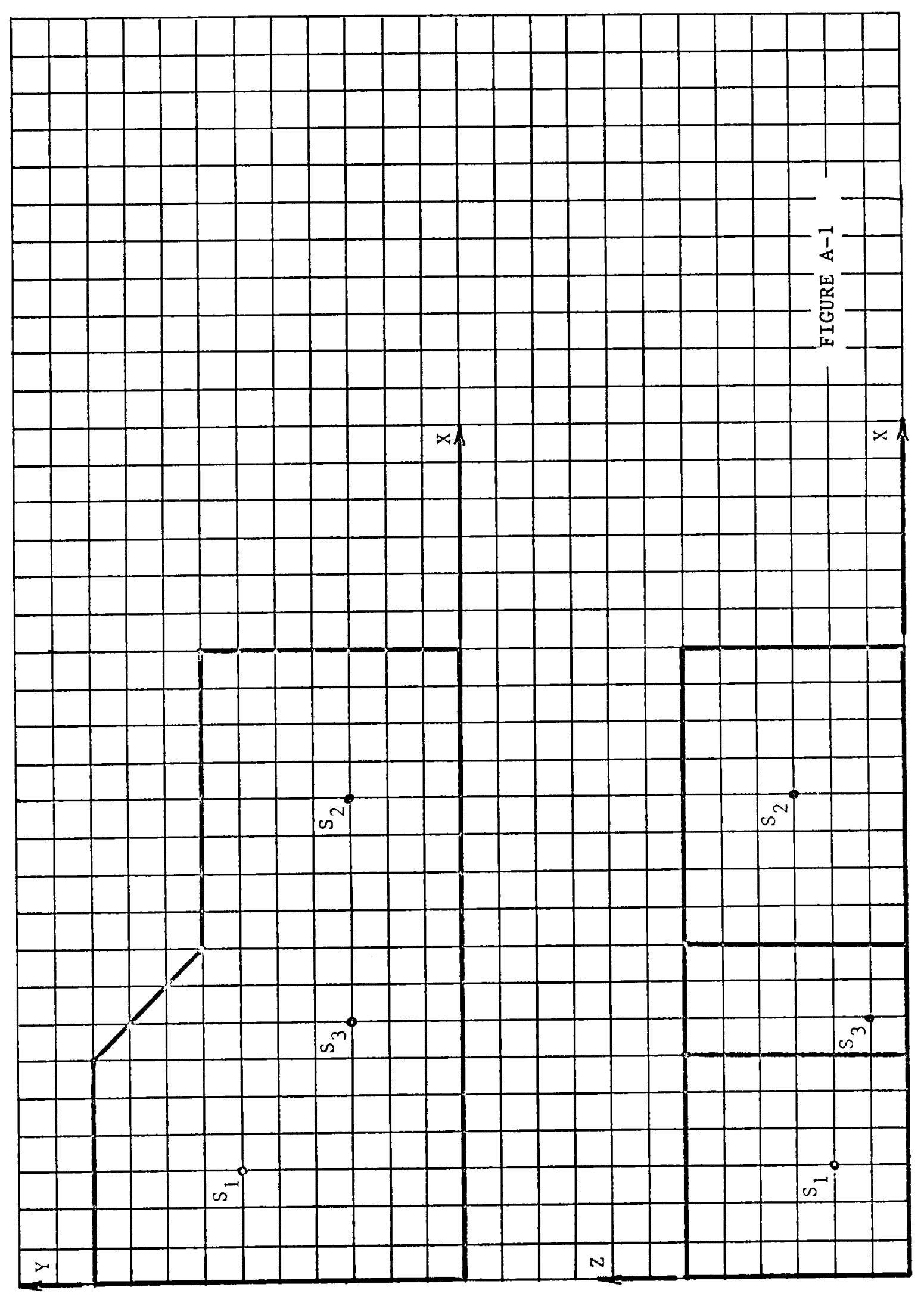


positioned in the first column. If no plot is wanted a zero goes in the first column. In all cases a plot of the room in three dimensions will be made to assure user that data was correctly entered.

The number of sources is entered in column five of the second data card.

On the third data card are entered the $\mathrm{X}, \mathrm{Y}$ and $\mathrm{Z}$ coordinates of the first source. The $\mathrm{X}, \mathrm{Y}$ and $\mathrm{Z}$ coordinates are entered in columns $1-10,11-20$ and $21-30$ respectively. The octave band sound power level (in watts) for the source whose location was given on the third data card is then entered in columns 1-10 of the next eight data cards, one per data card beginning with $63 \mathrm{~Hz}$.

The preceding procedure is repeated for each source, e.g., the coordinates of the source are entered followed by its octave band sound power level spectrum.

After the data on all sound sources has been entered the number of surfaces is entered on the next data card in column 5 .

The first surface to be entered must be the floor.

The number of end points (corners) of the floor is entered on the next card in column five. An end point is defined as the point where three surfaces intersect.

The absorption coefficients followed by the corner points of each wall are entered as follows. 
The octave sound absorption values of the floor are entered on the next eight cards in columns $1-10$, one per data card beginning with $63 \mathrm{~Hz}$.

The $X, Y$ and $Z$ coordinates of each corner point of the floor (surface) are entered starting at any corner and proceeding clockwise or counter-clockwise around the wall boundary until all corner points are entered. Each coordinate set is entered on a separate data card in columns $1-10,11-20$ and 21-30. This procedure describes a plane. Repeat this procedure for the ceiling and walls.

If a surface contains windows, or patches of absorption as shown in Figure $\mathrm{A}-3$, it must be divided into segments so that there are no interior boundaries completely enclosed. Figure A-4 illustrates one method of segmenting the wall surface so that no interior boundaries are completely enclosed. The numbers indicate the number of walls to be entered. As explained in the preceding instructions. When one wall surface is subdivided into several smaller areas the absorption coefficients will be entered just as if it were a separate wall.

The last card contains seven entries. The desired increment of the receiver location is entered in columns 1-10. If a zero is entered then no incrementing will take place. The X, Y and $Z$ coordinates of the initial receiver position are entered in columns 11-20, 21-30 and 31-40 respectively. The final entries on this card are the maximum $X, Y$ and $Z$ dimensions of the factory space. 
The computer output will include the coordinates of the receiver position, the octave band sound pressure levels, and the overall sound pressure levels. If a ray tracing plot is requested the plan and elevation views of the rays will be plotted. 


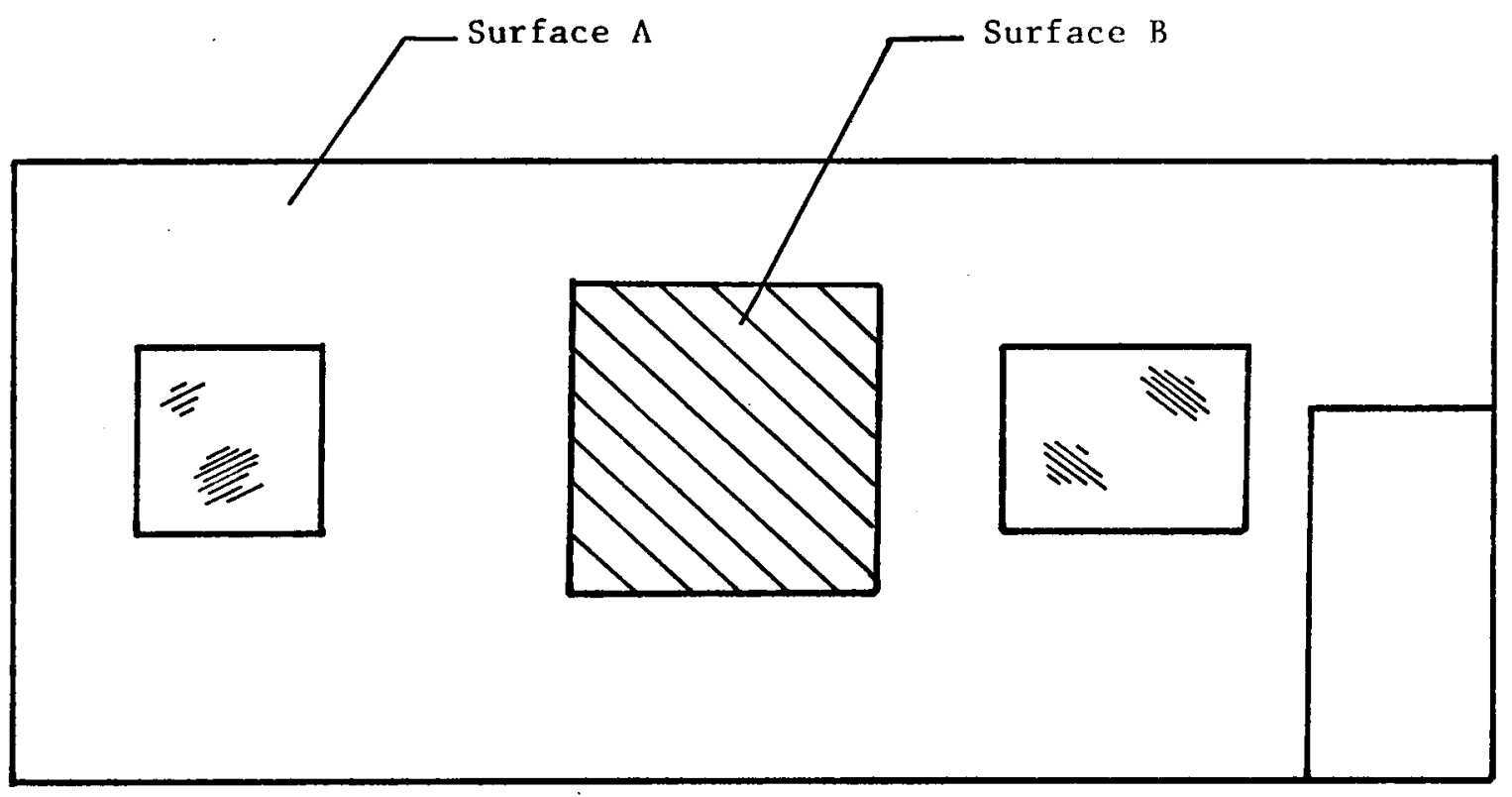

Surface "B" is completely enclosed within Surface "A".

FIGURE $\Lambda-3$ 


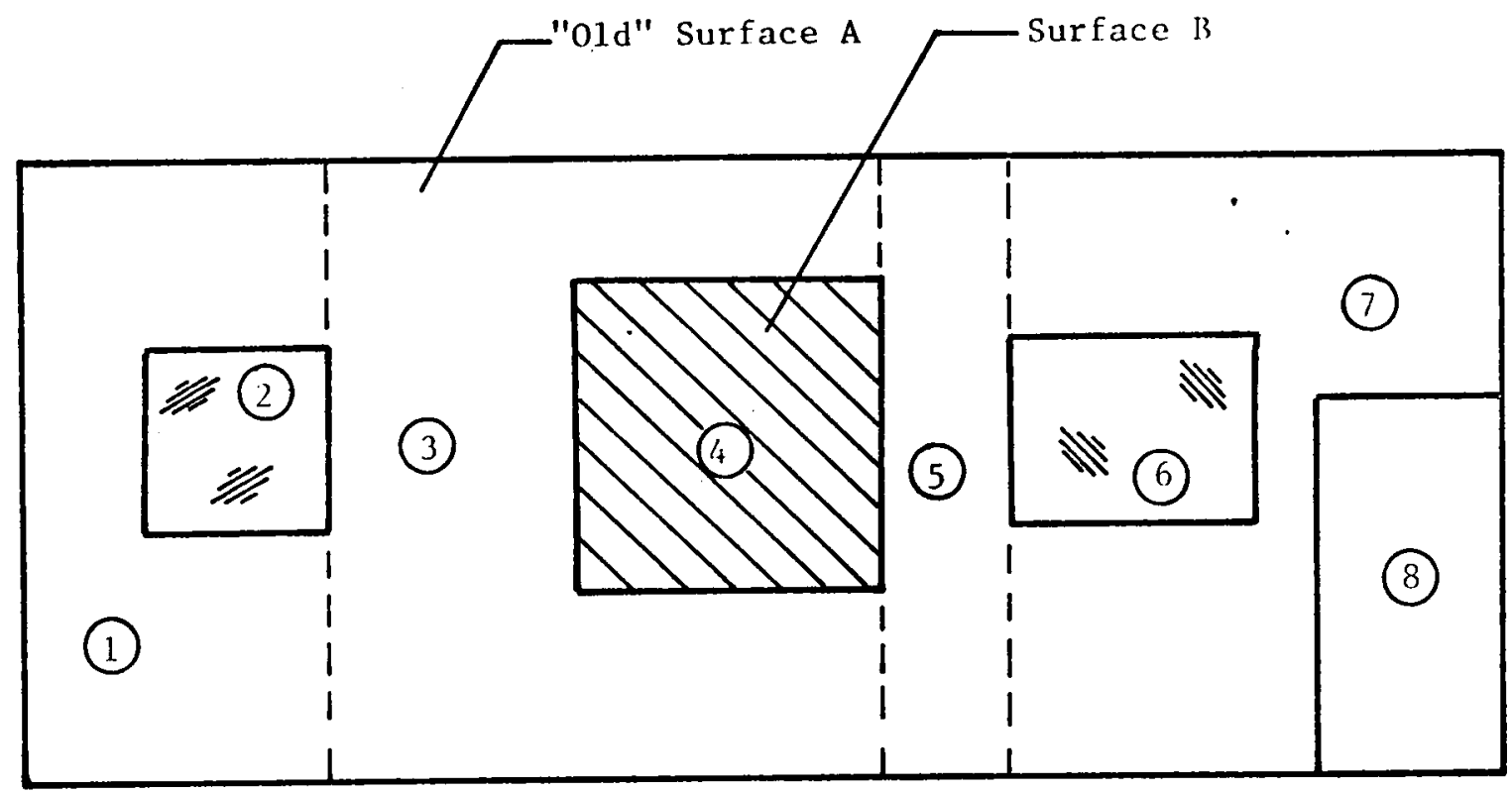

Surface " $B$ " is not enclosed by "old" Surface " $A$ " but is adjacent to new Surfaces " 3 " and "4".

FIGURE $\Lambda-4$ 

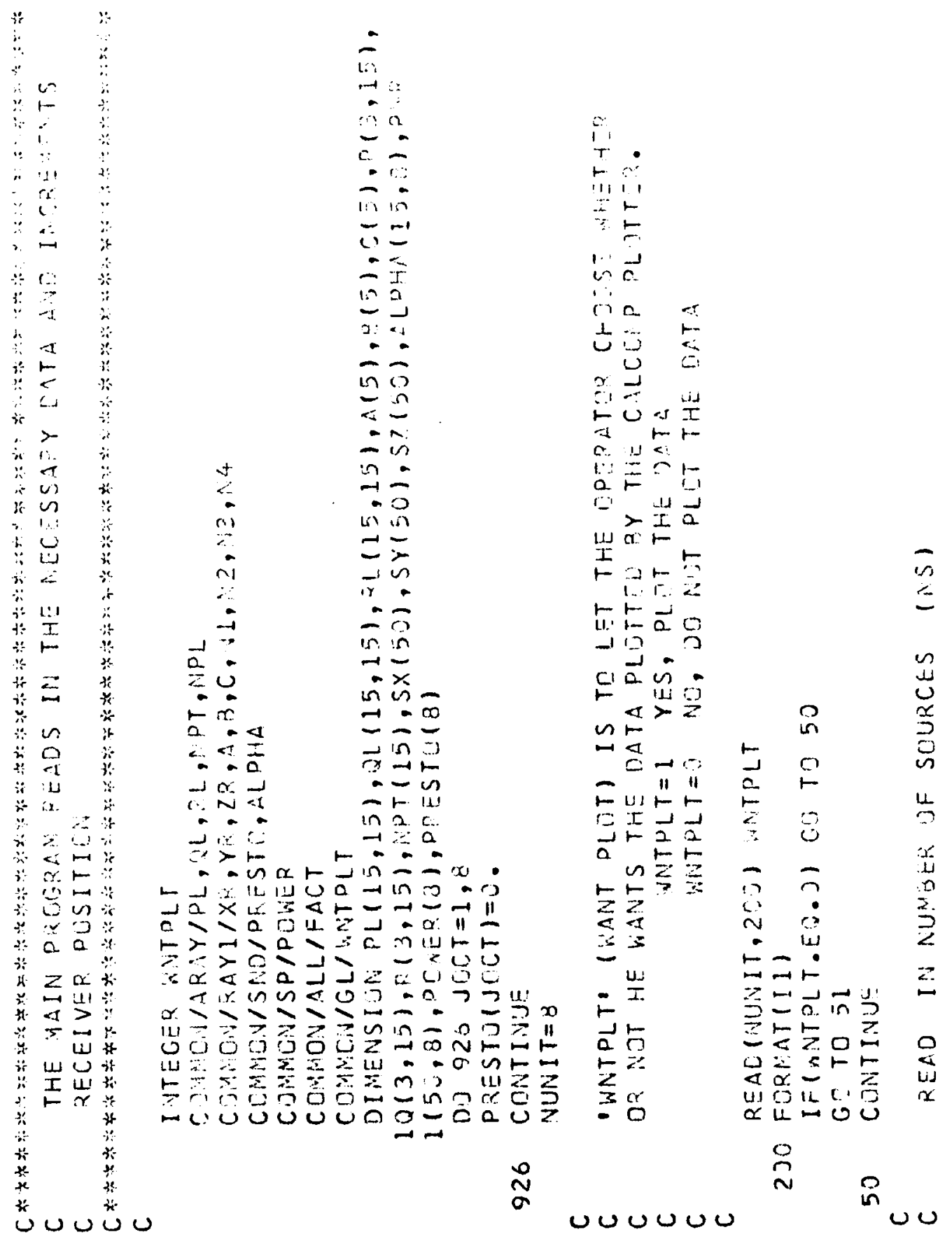


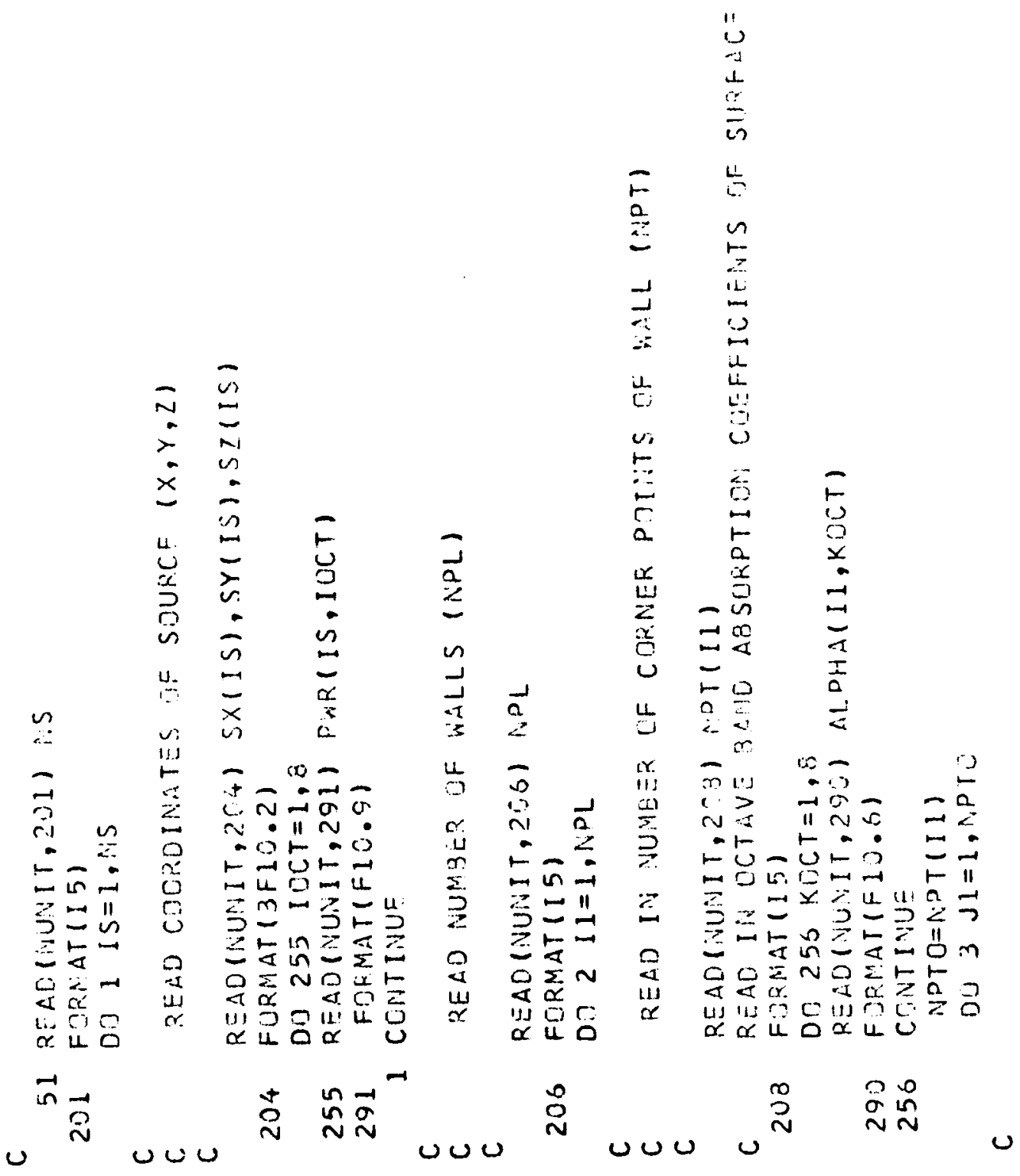



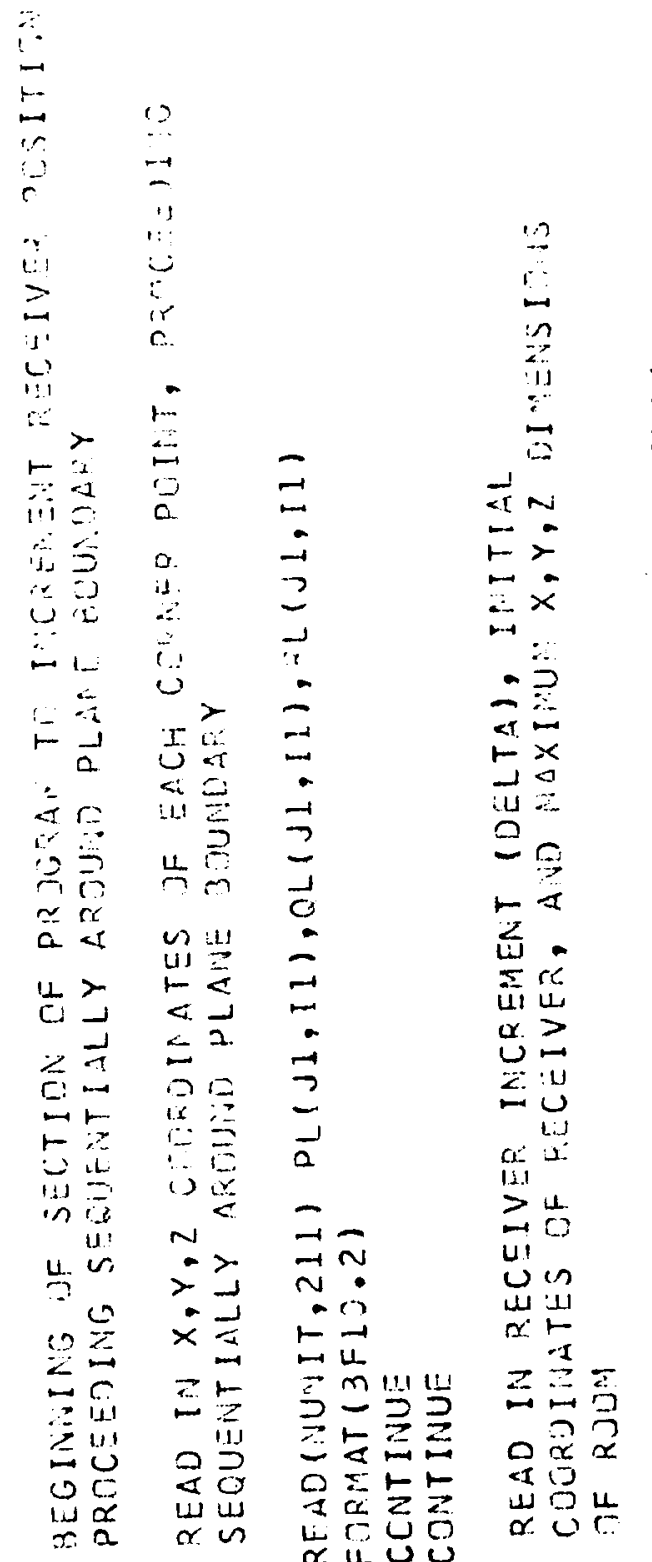

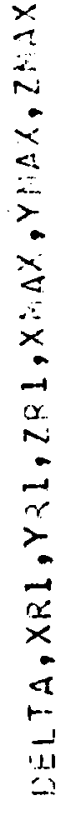

$\vec{a}$

$\rightarrow$

$\vec{N} \cong$

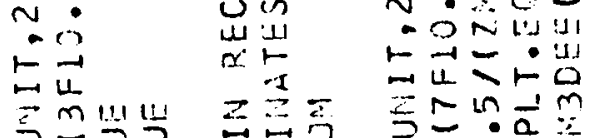

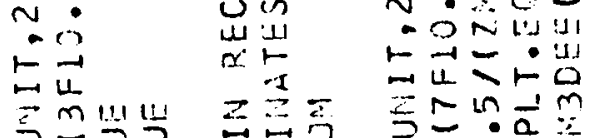

こ?

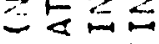

里进至

近出包

《I (⿹

Z二

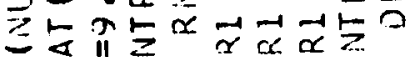

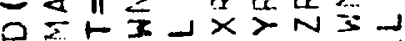

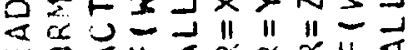

出

$\frac{2}{2}$

$\therefore 5$

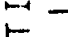

$\rightarrow$ is

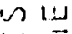

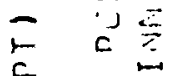

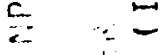

$\Rightarrow$

$=$

$\exists$

1.

in

I:

$>$

$\geqslant u$

is

世亭

Os

$\alpha x+$

$a$

미 I $x-\frac{1}{x}$ $20=$

$+3$.

$m-x ?-1$

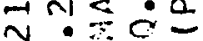

$\stackrel{0}{\circ}$ 


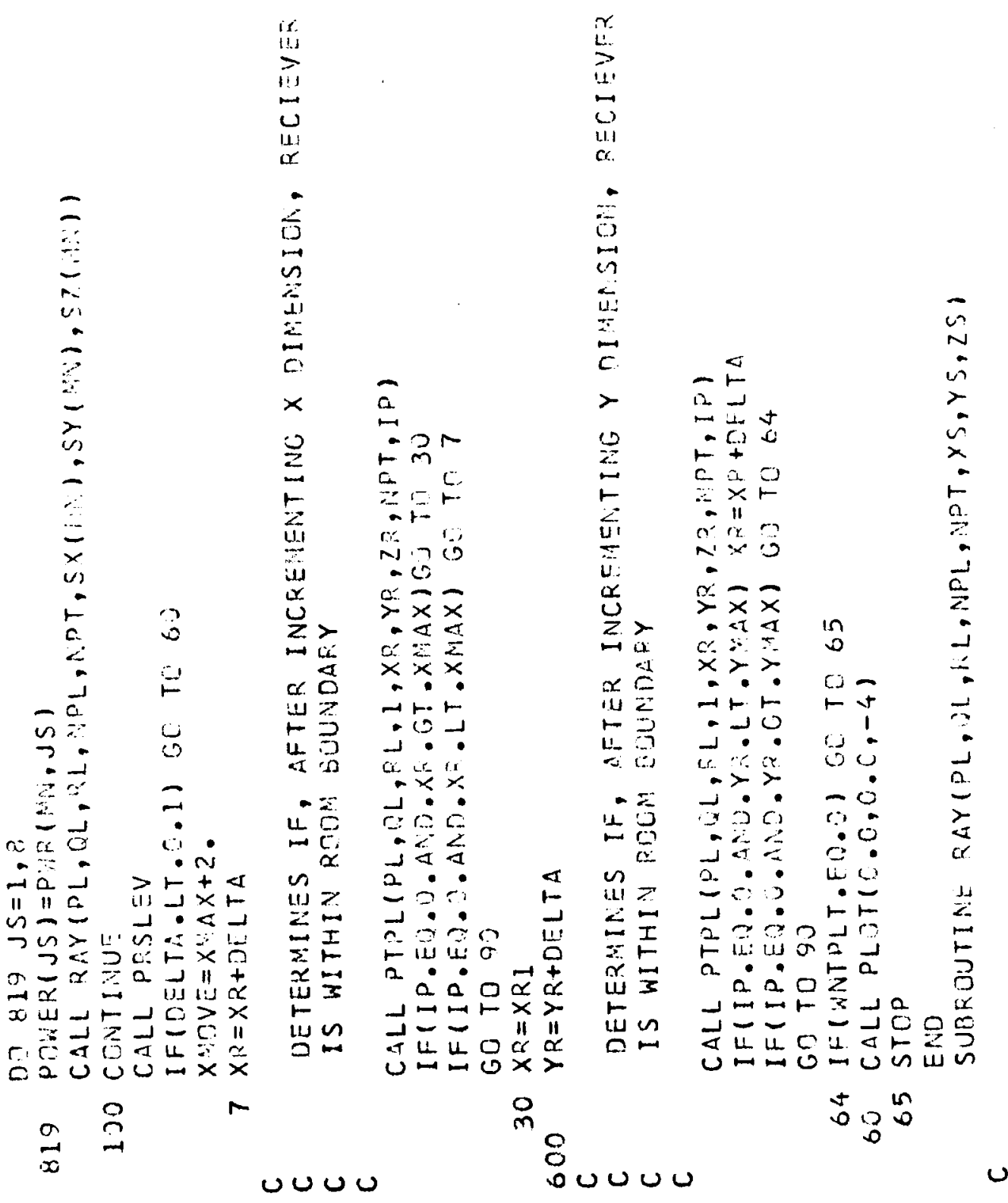



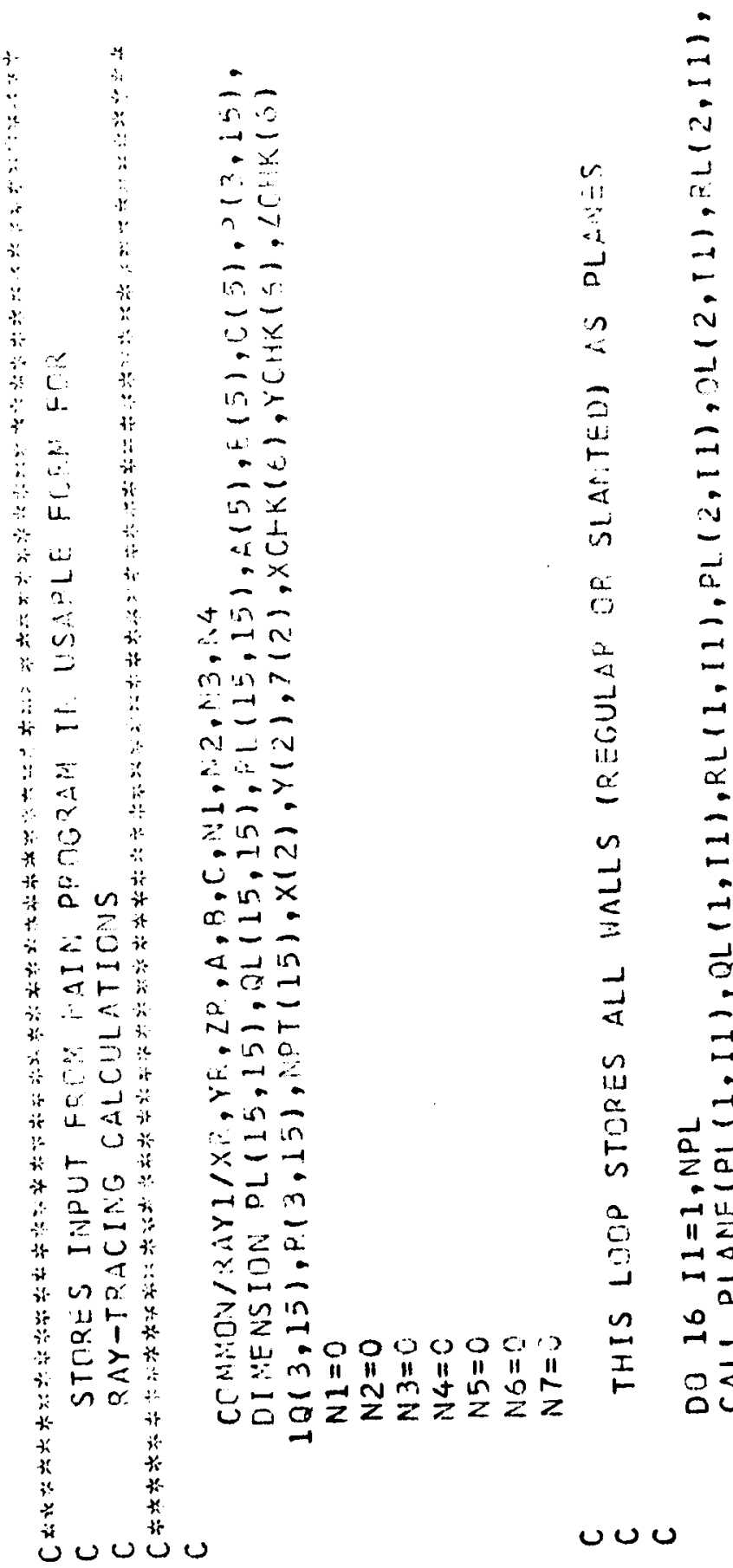

$a \quad \div$

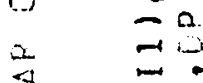

$j_{0}^{1} \quad=20=$

i.i.

a. a a

$\underset{x}{x}$

n IASO

$\underline{J}$

$\sim$ a

$\therefore=-2$

$-\not \dot{0}: \dot{c}$

$\vec{\theta} \dot{0} \dot{0} \dot{c}+\mathrm{n}$

$\pm$

- MLilita-

- - . .

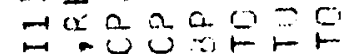

- -1

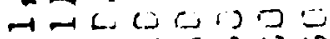

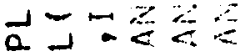

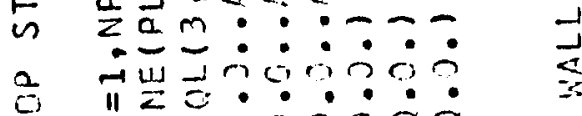

$\Rightarrow \quad-4-0 ; 0000$

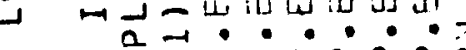

$\sim \quad 0$ ma $a \circ a 2 a$

$\rightarrow-1-\infty=4<100$

I 0 a

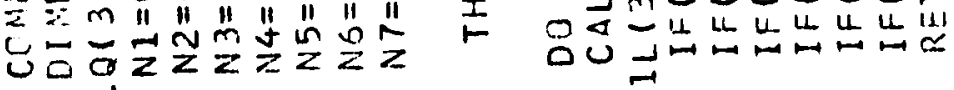

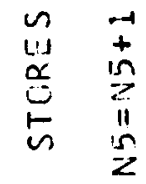

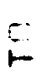

4

$\Xi$

?

c.

- it 


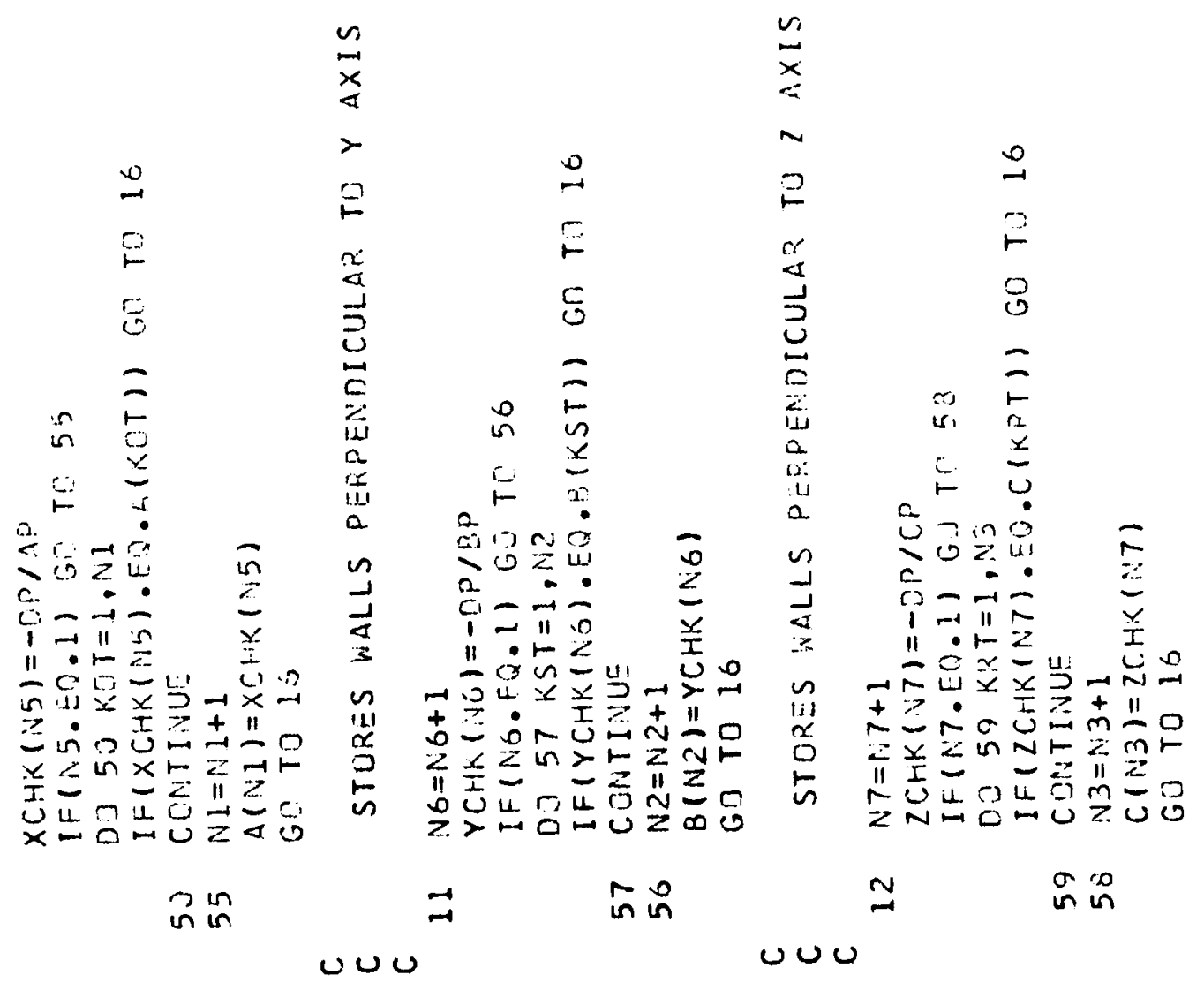




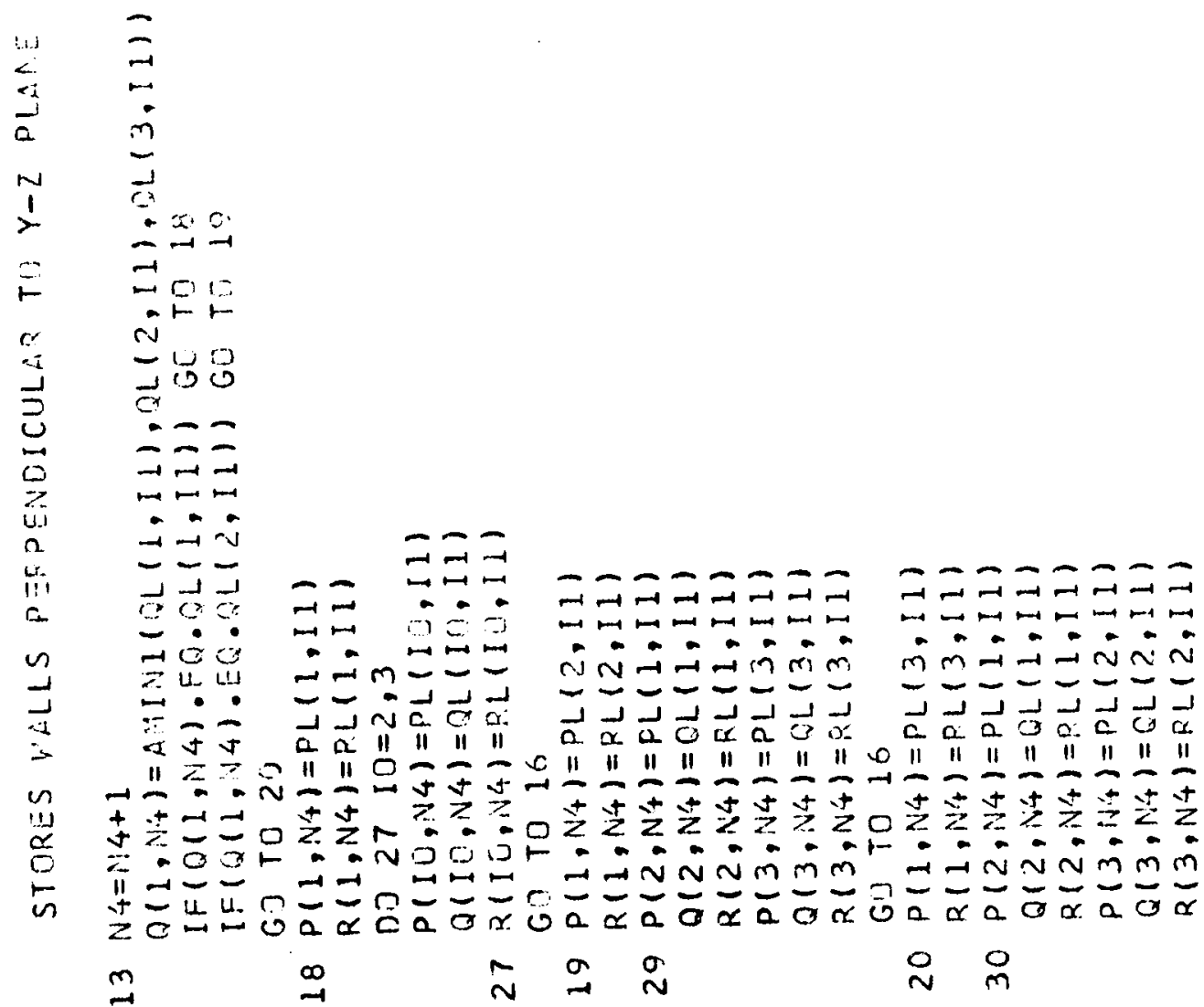

$\omega 0 \omega$ 


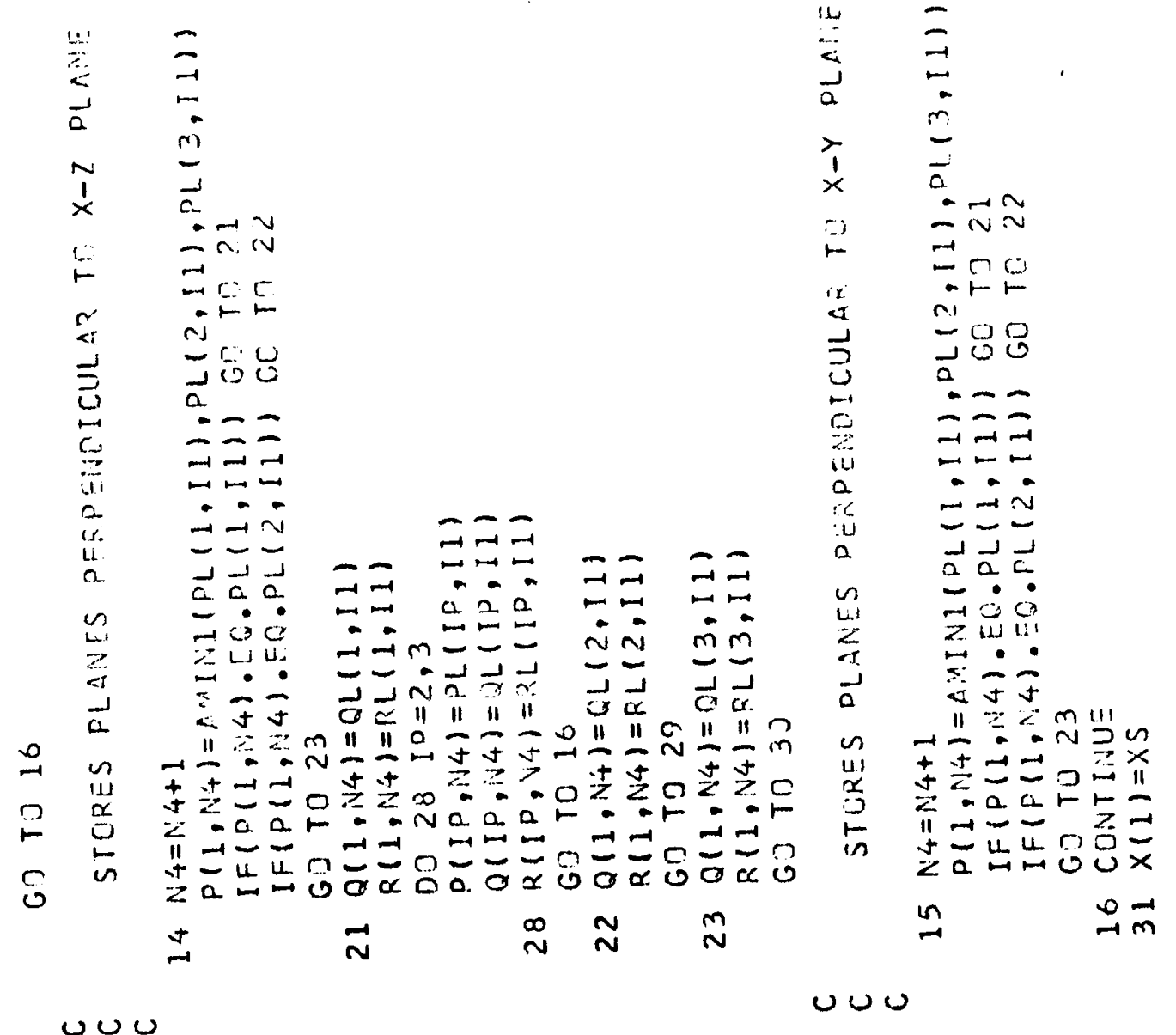




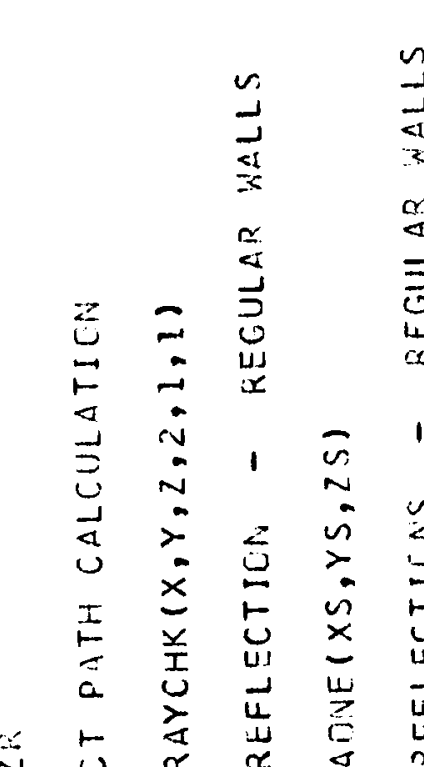

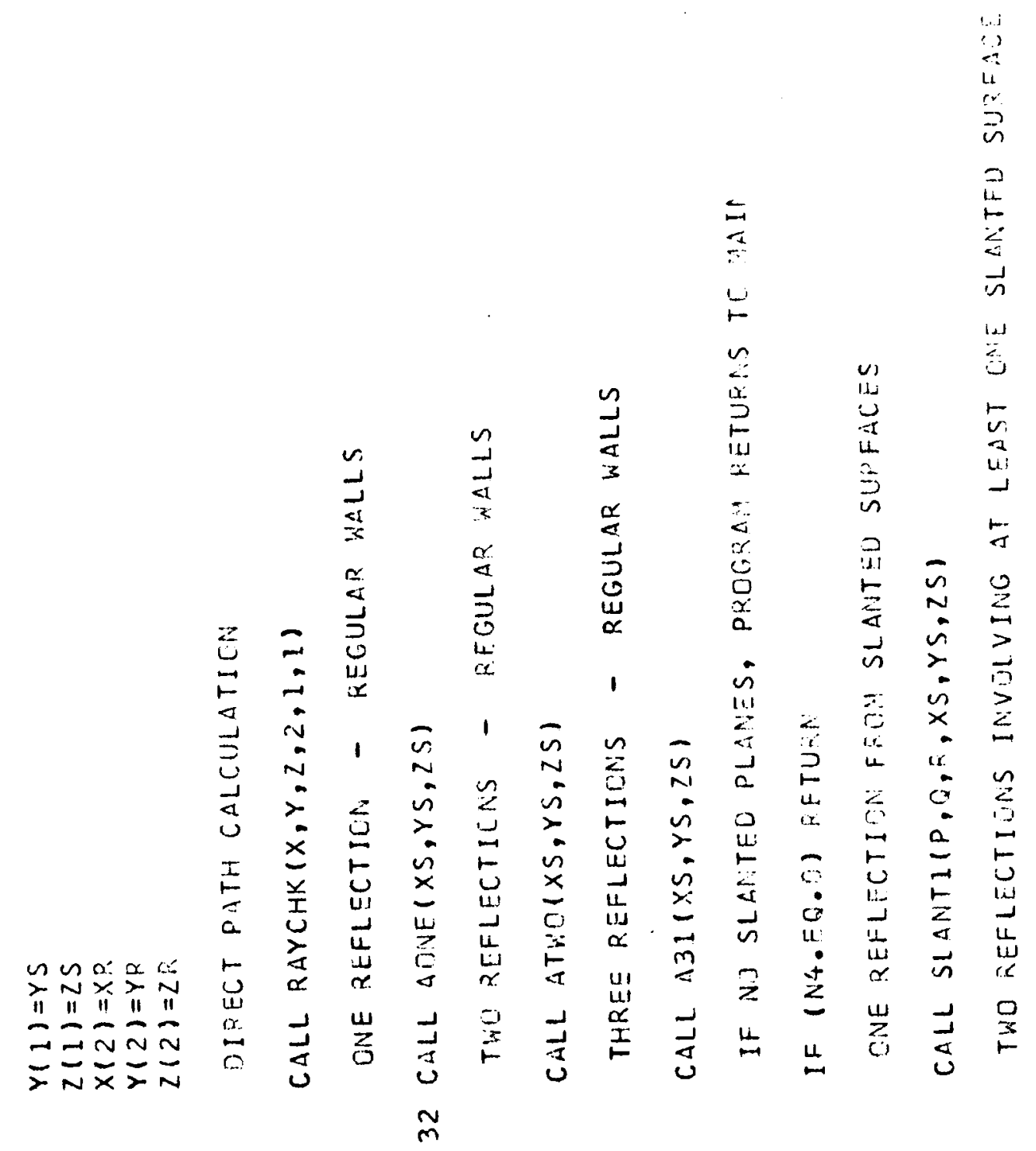

טง 


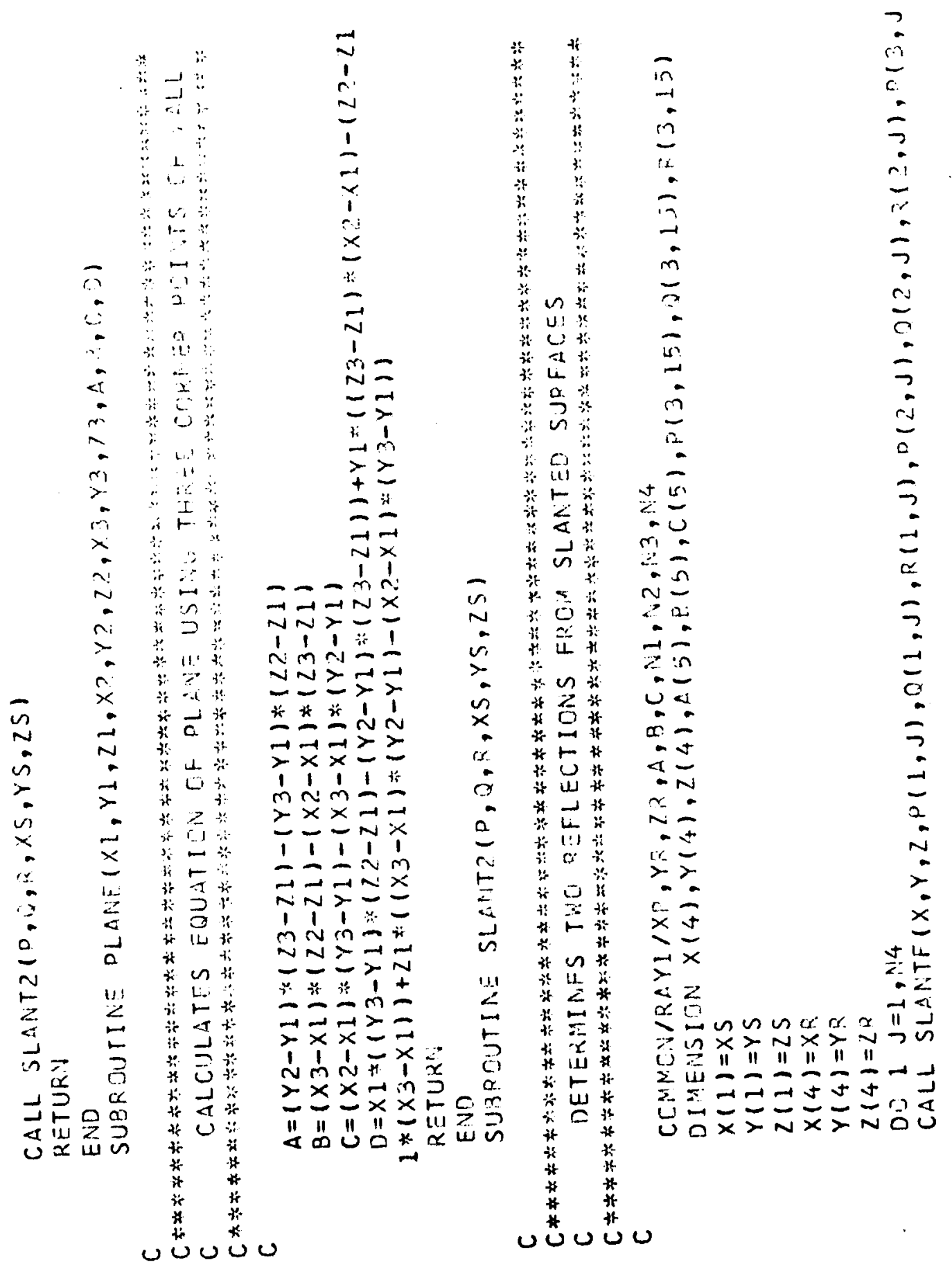




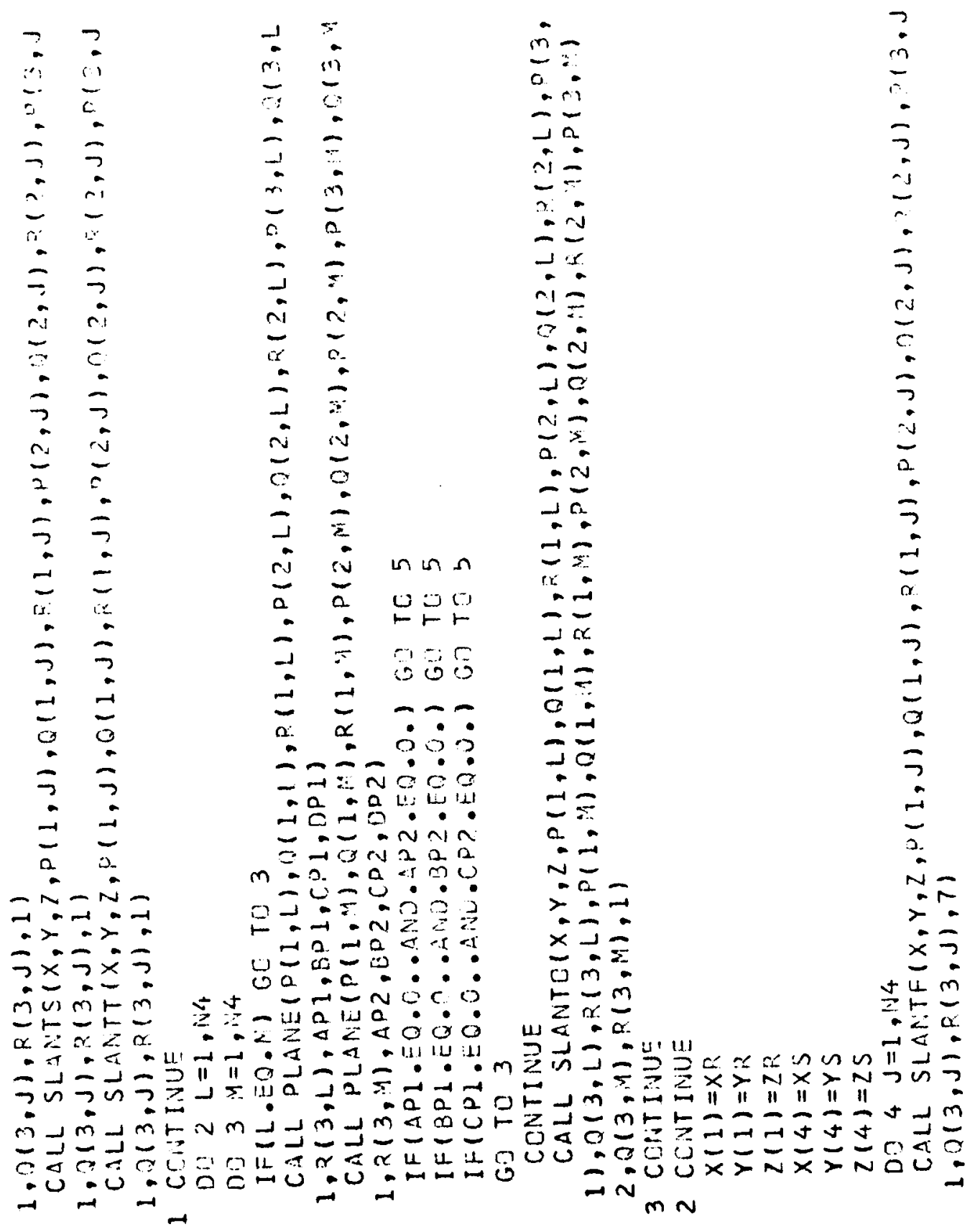

in 


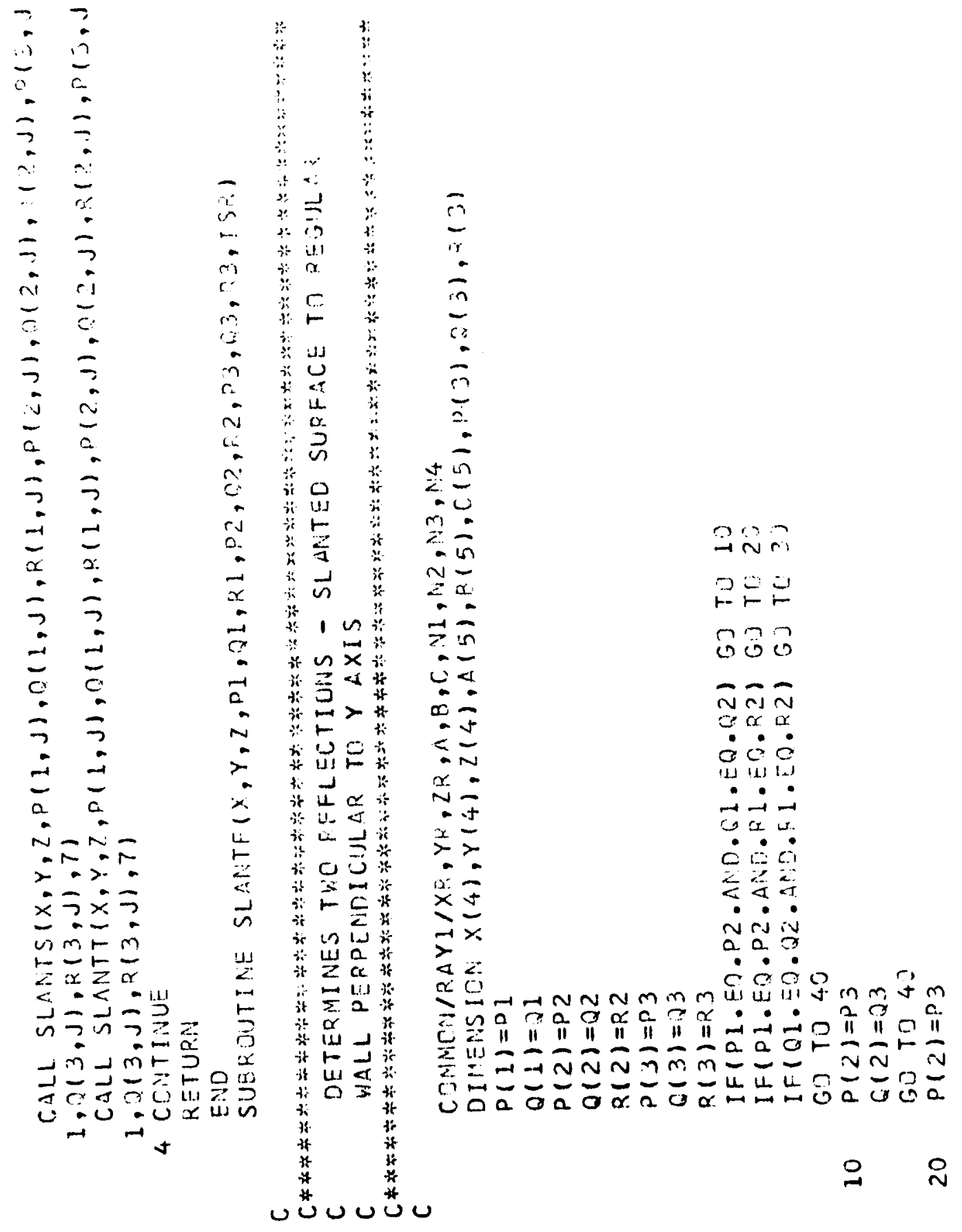




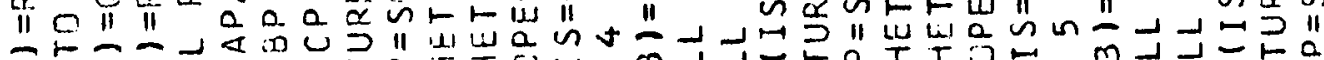

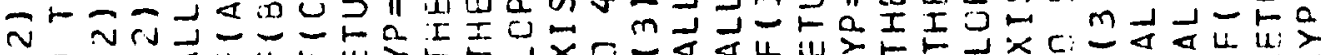

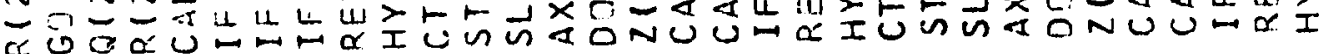




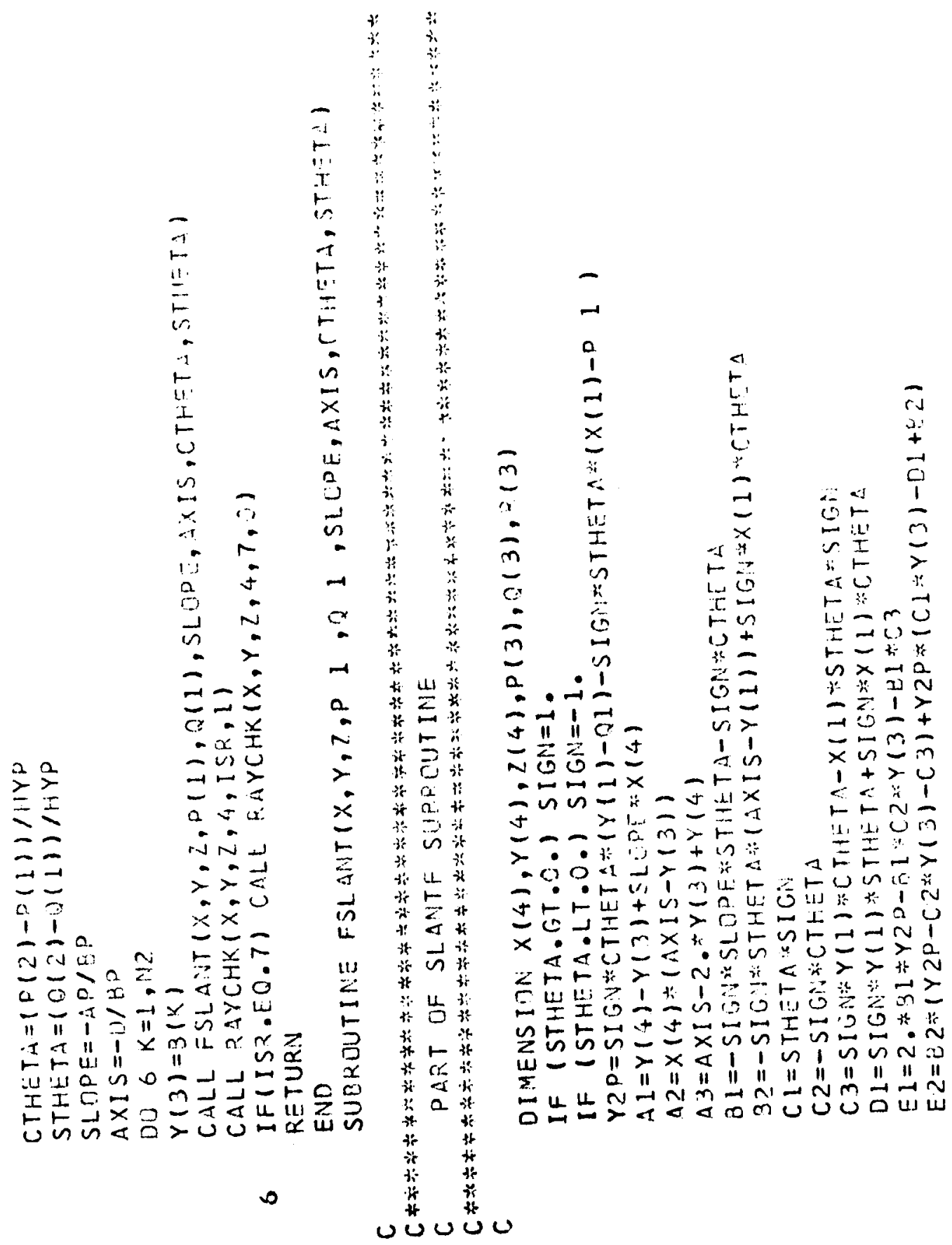




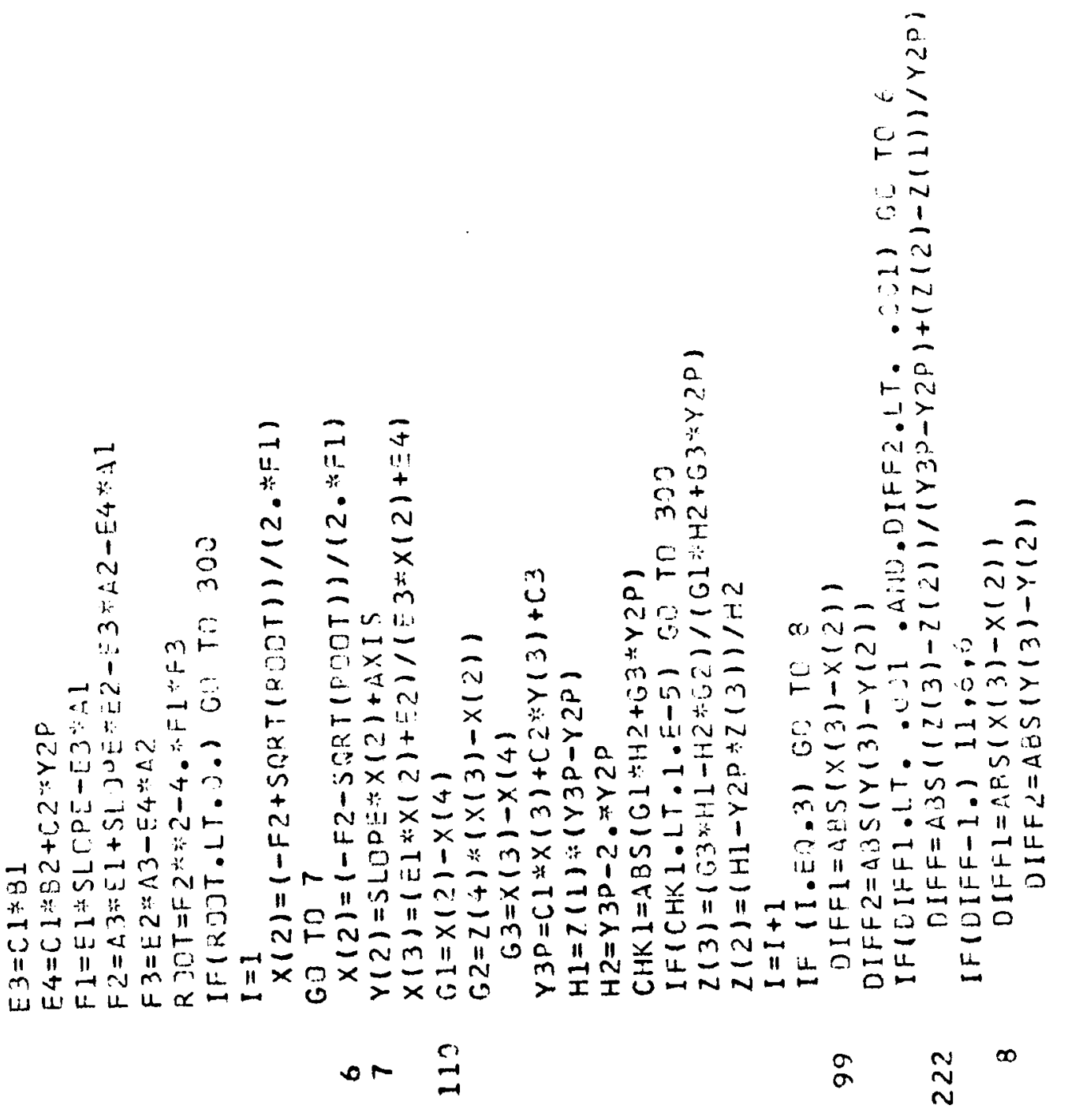




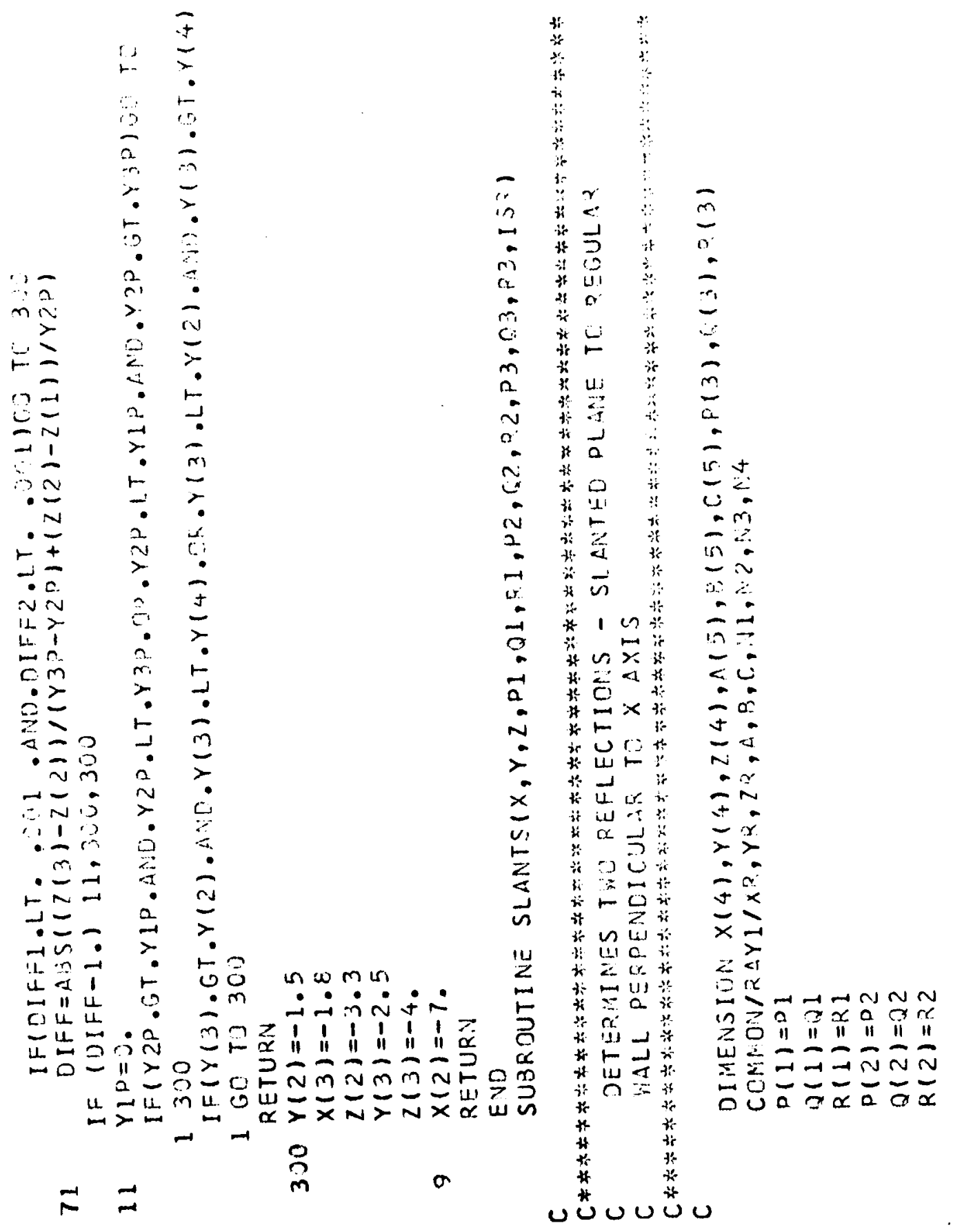


and

Dே

की०

$\bar{N} \bar{v}$

$0 x x$

$\dot{3} \dot{0}$

1.1. is it

$\rightarrow-1$

Cir

$\dot{0} 0$

要运

$4 \pi<$

N $\sim$

00.0

$\dot{0} \dot{0}$
3

$\because$

3

$m_{1}$

is.

$m$

2

a

i

cr.

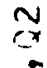

$\stackrel{i}{a-N m}=\frac{a}{2}$

$a n m$ 更工

aER $1=2$

다드

G०0 $00=$

$-0.00$

$\rightarrow$

$2 \div \div$

ن்0

$\because ?$

4000
$=14$

ज牲

w

$\angle-ニ=n$

-cirau

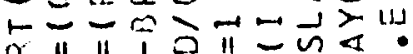

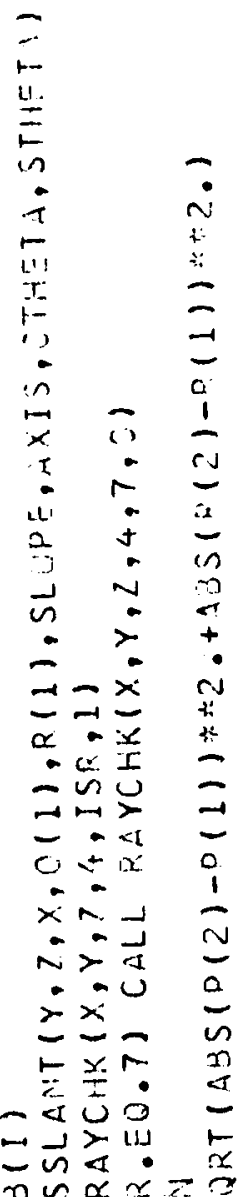

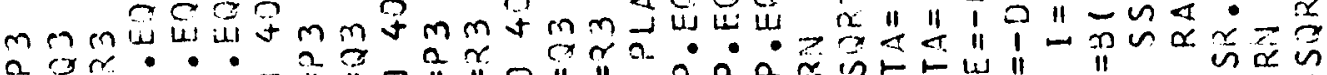

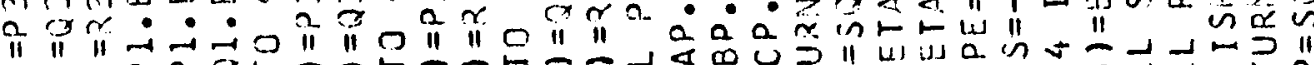

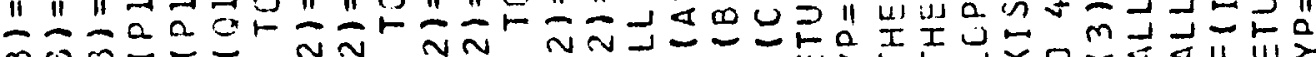

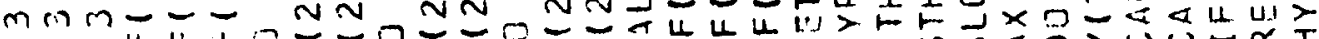

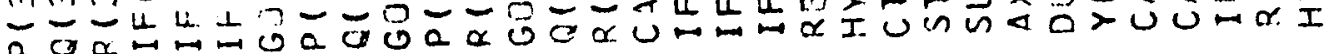

요요 


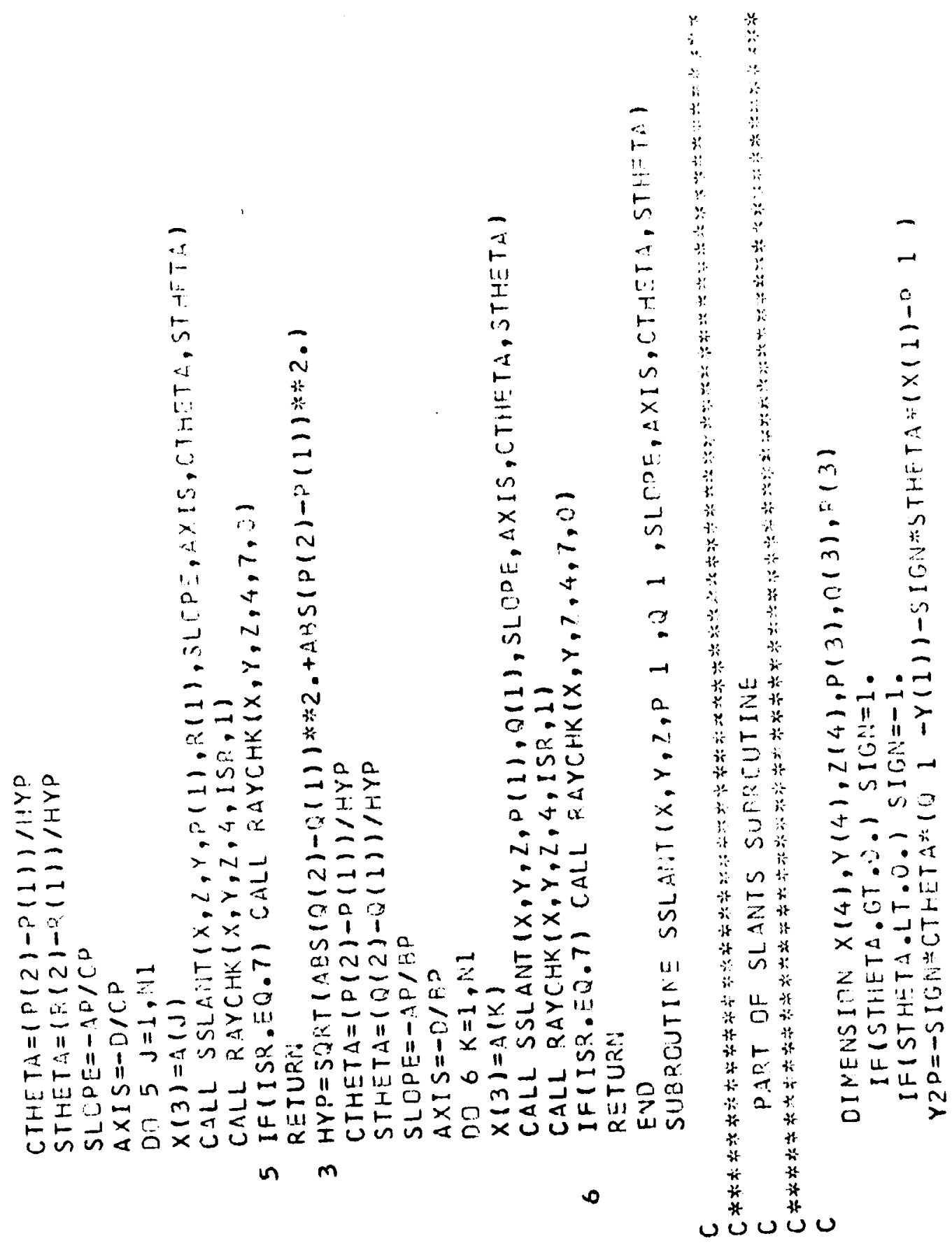




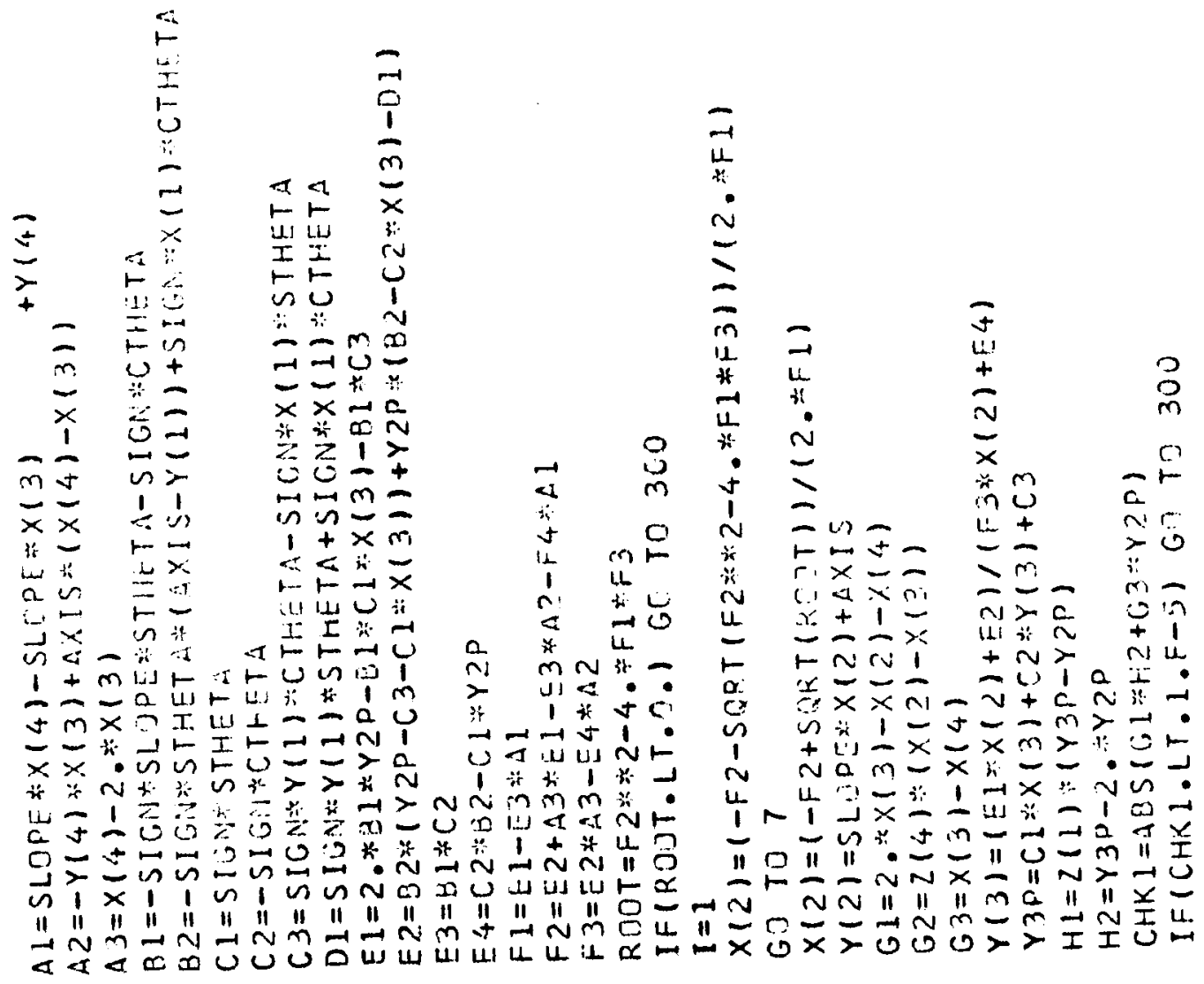




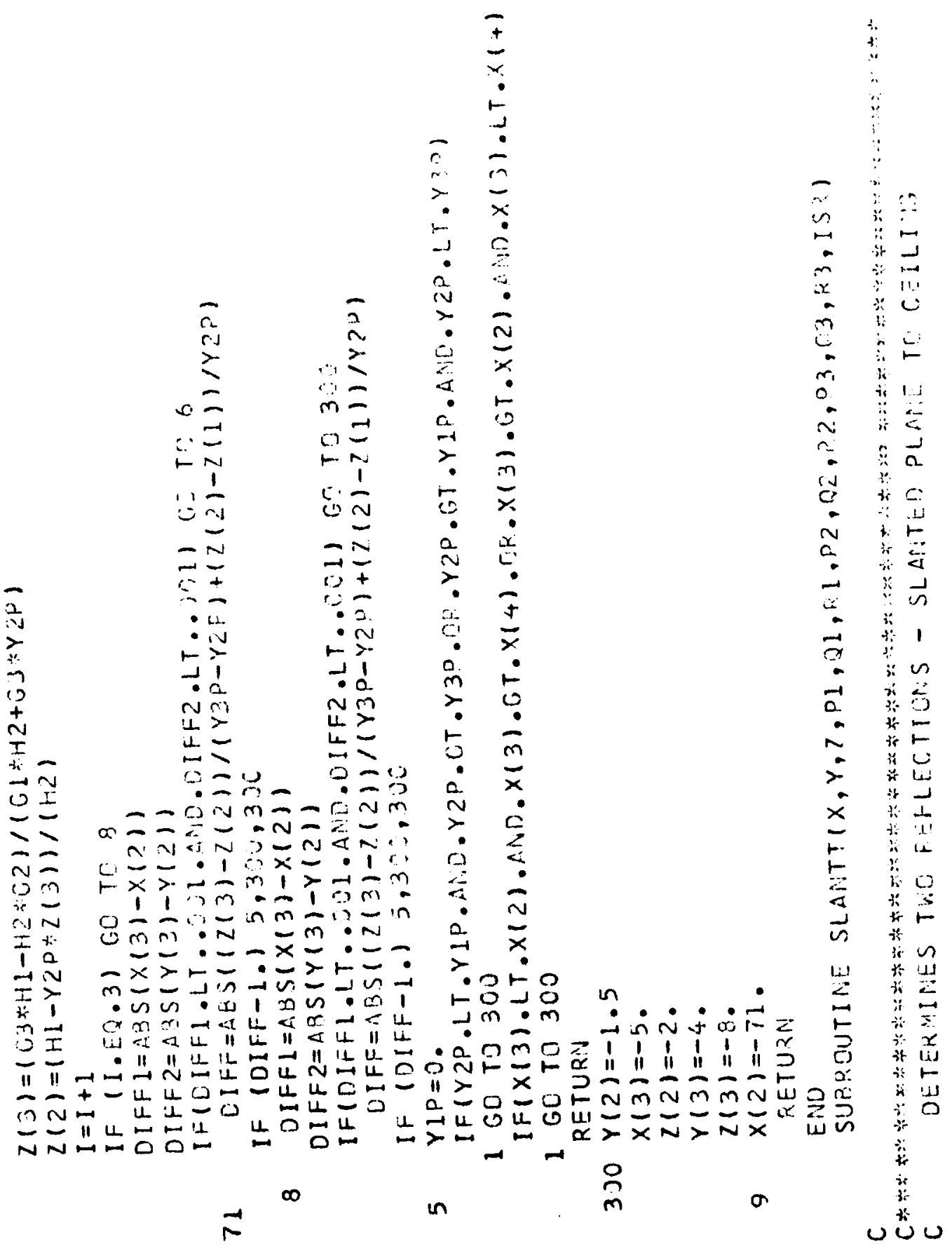



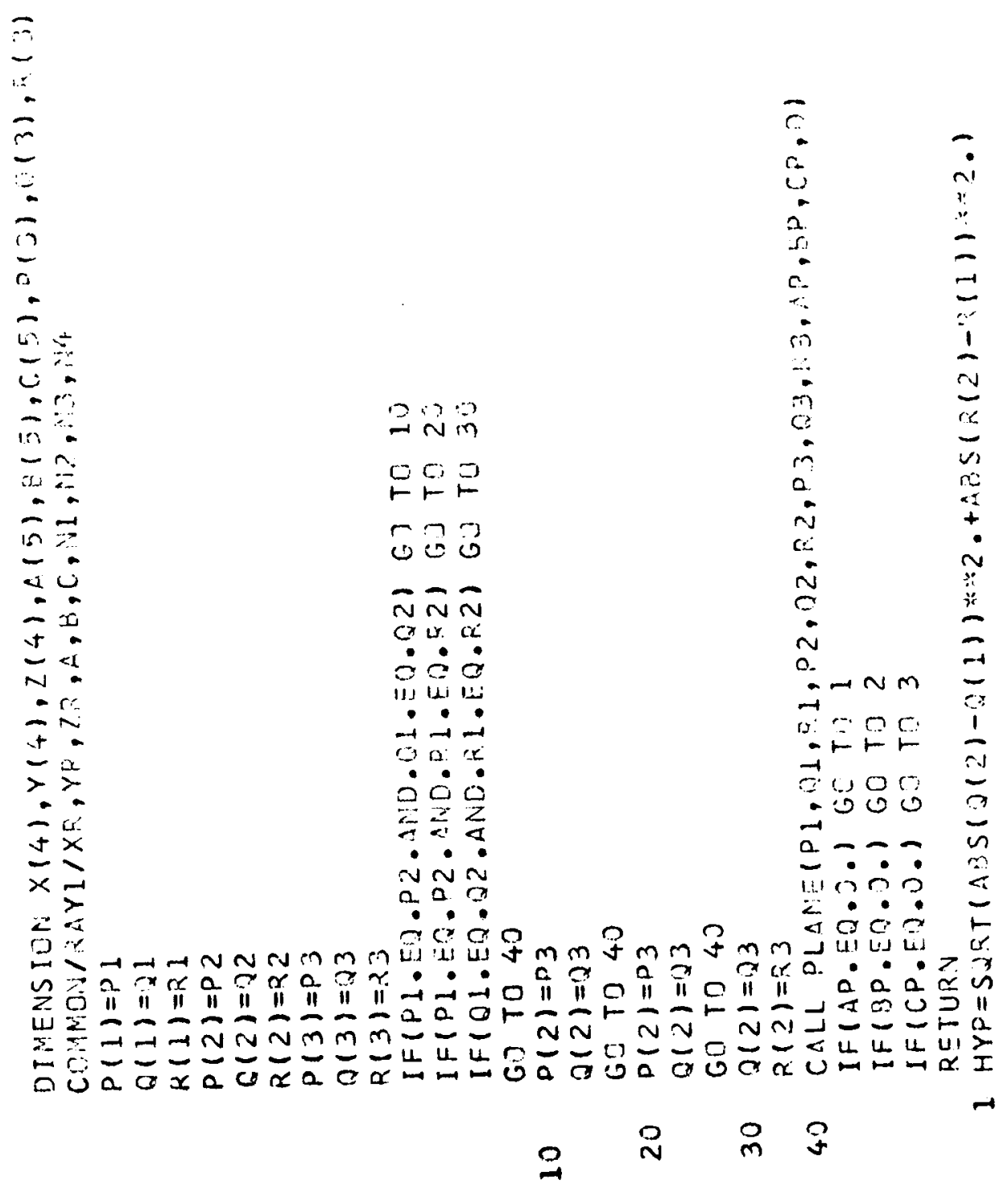


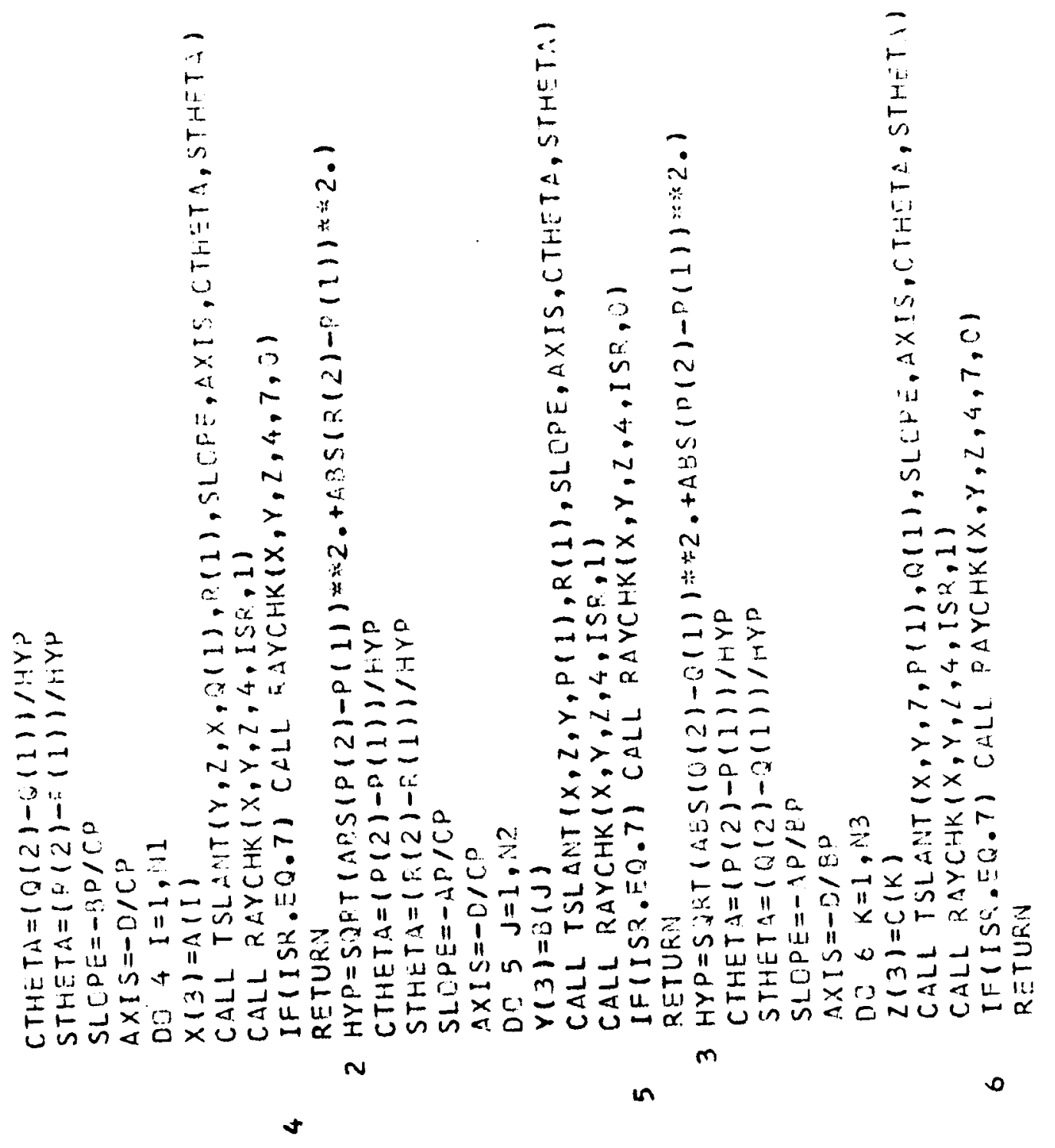




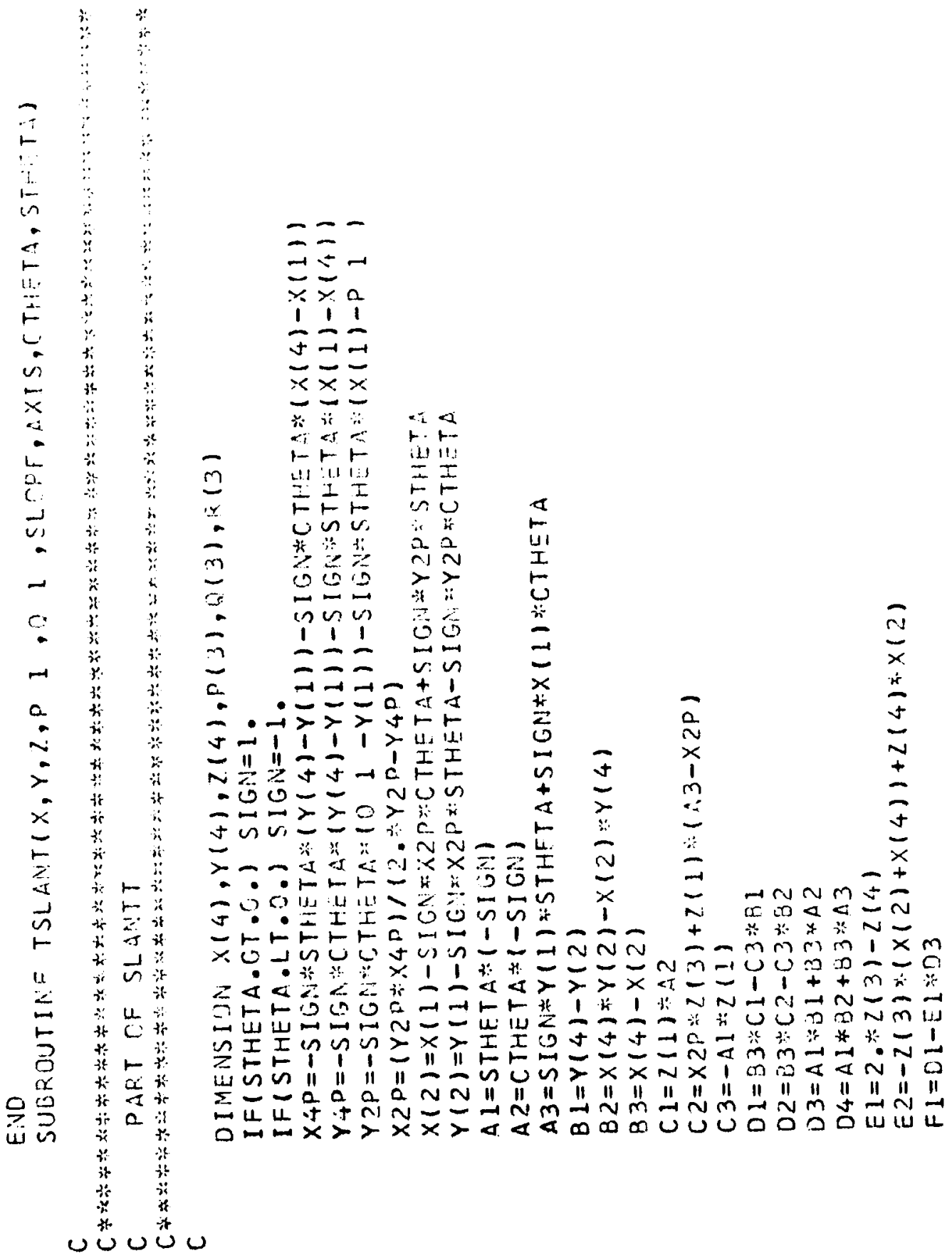




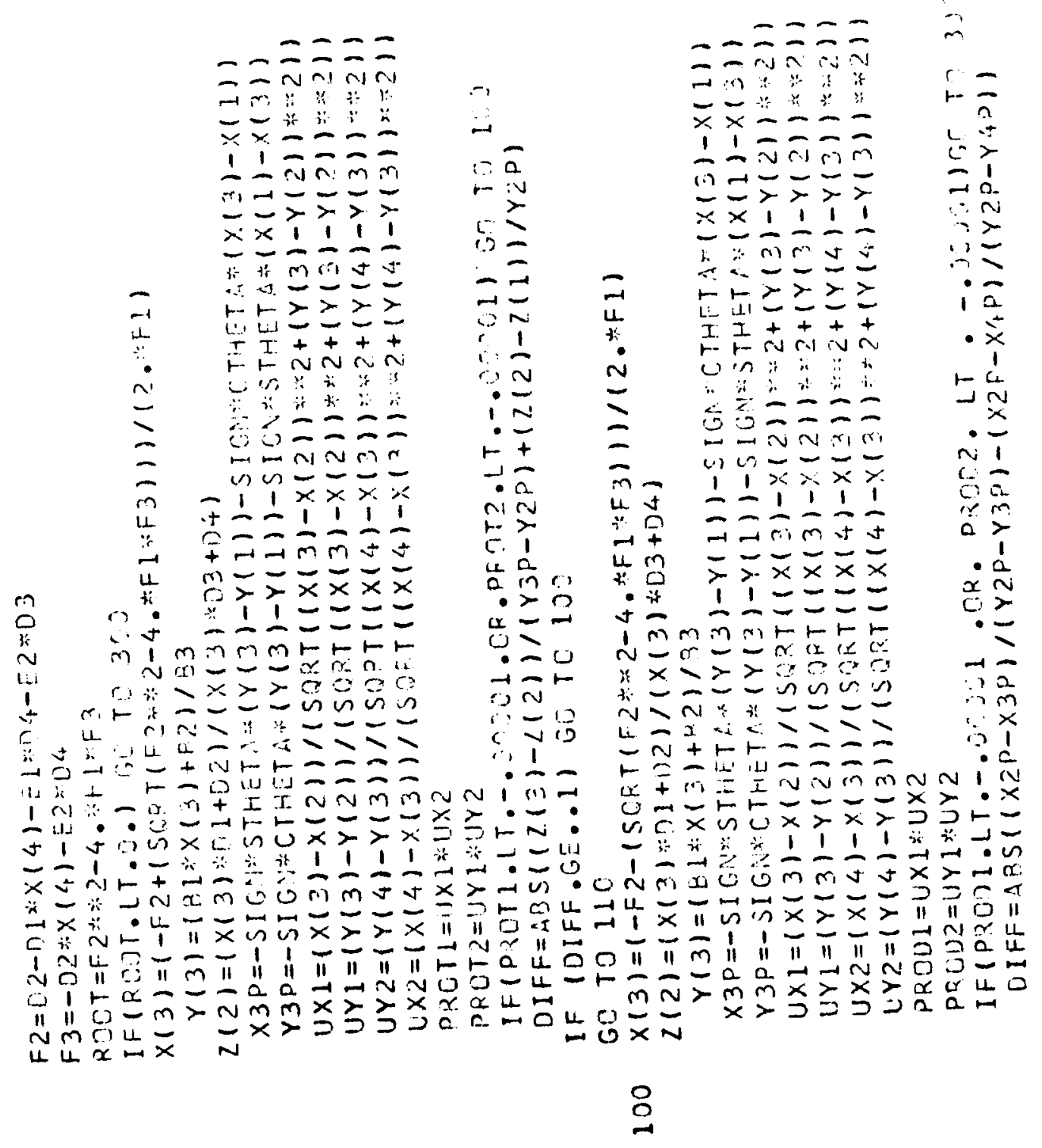




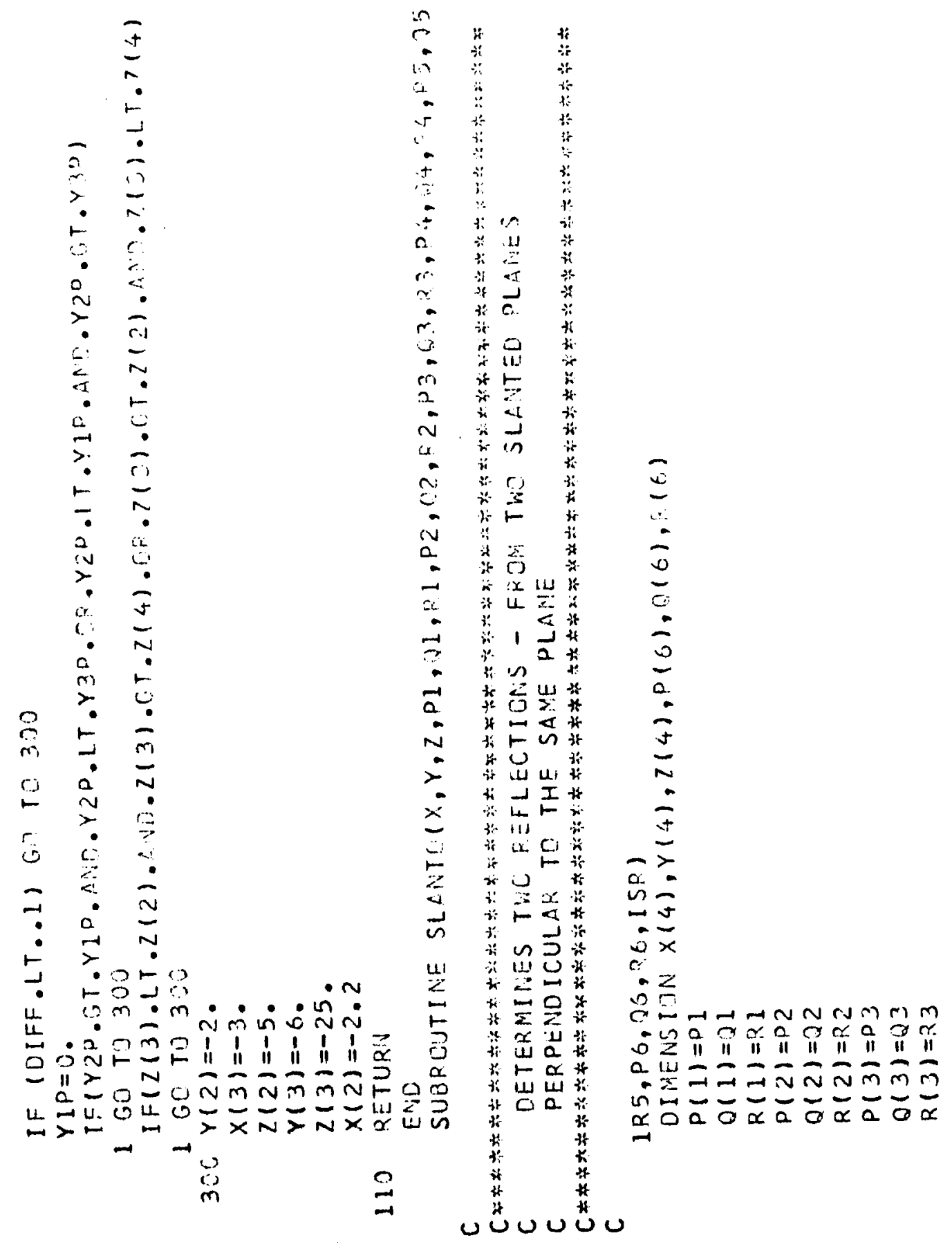



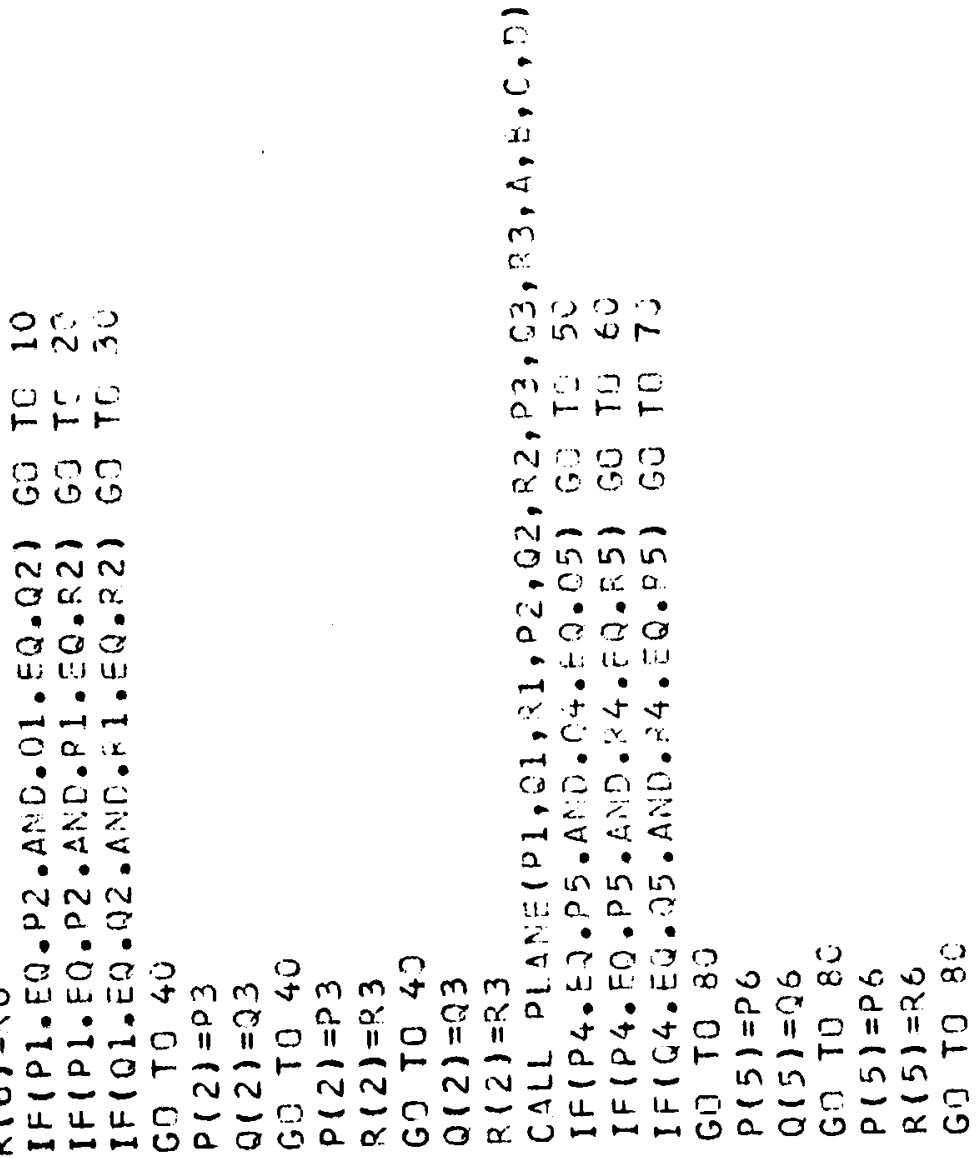
i1 il

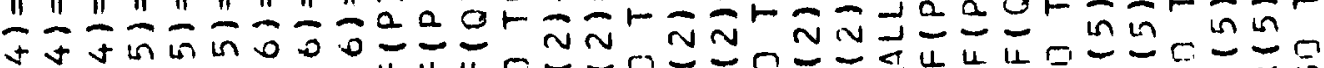

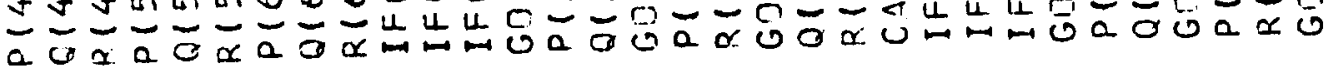

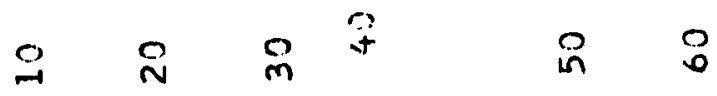




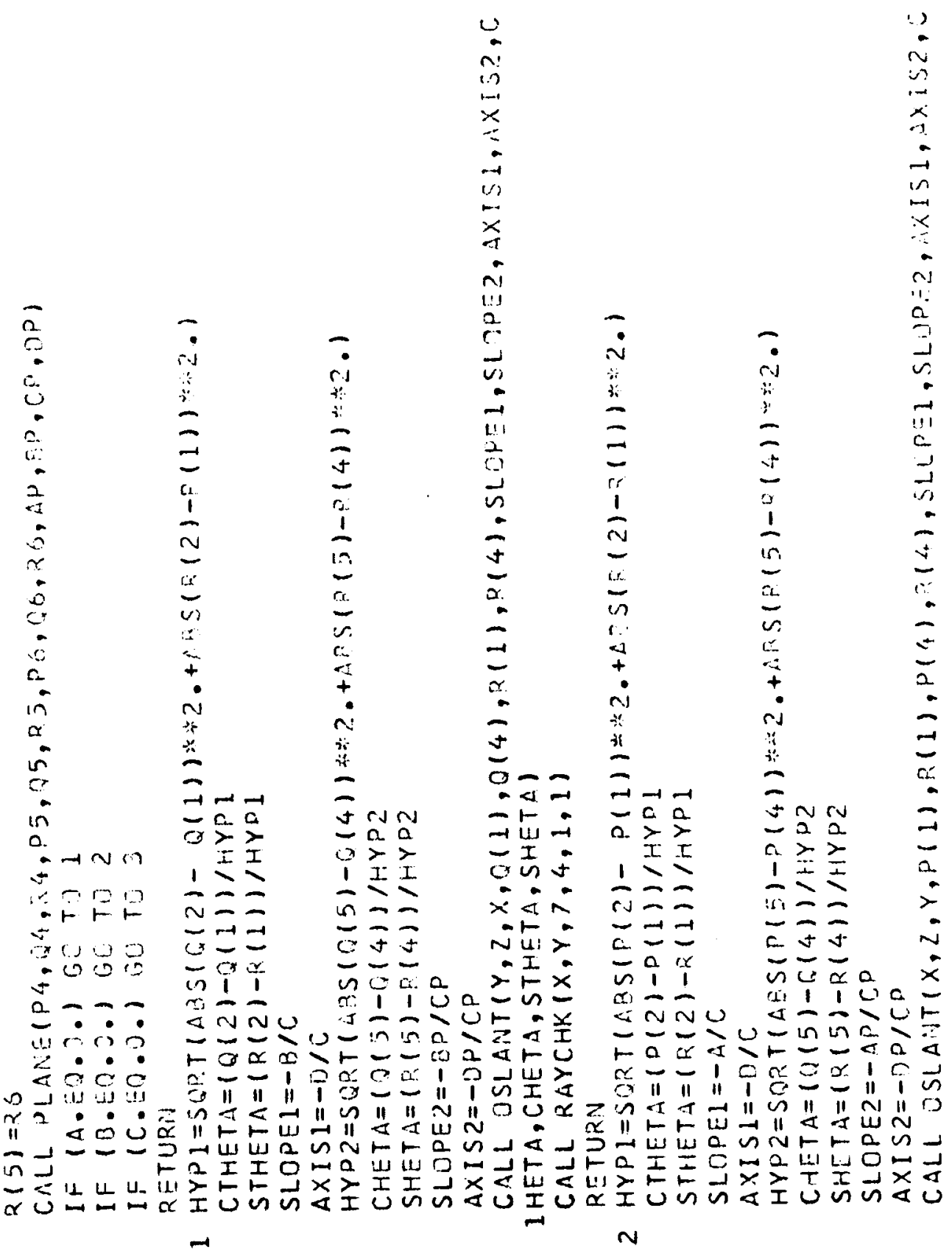

$\circledast \quad$ 







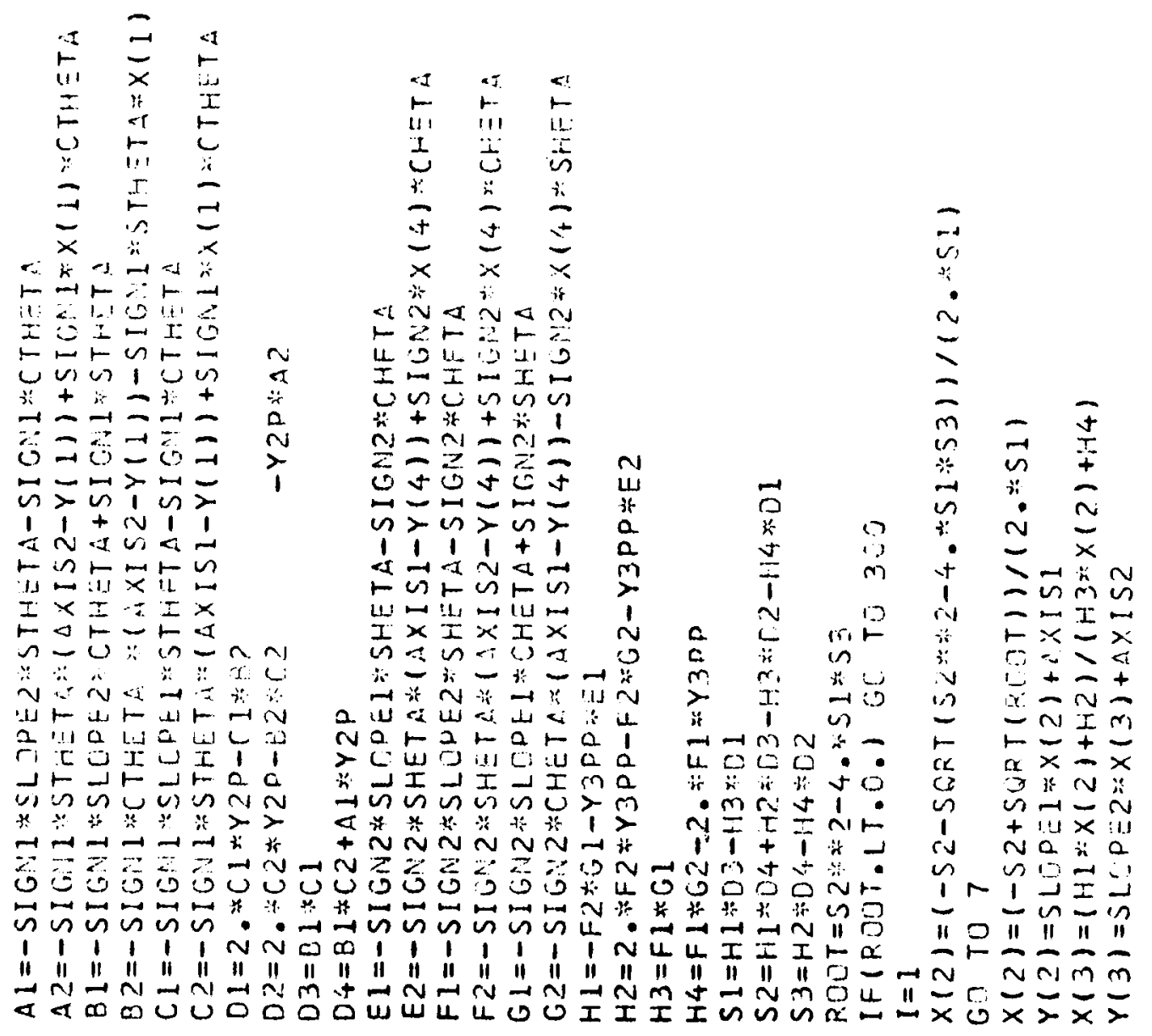

0 


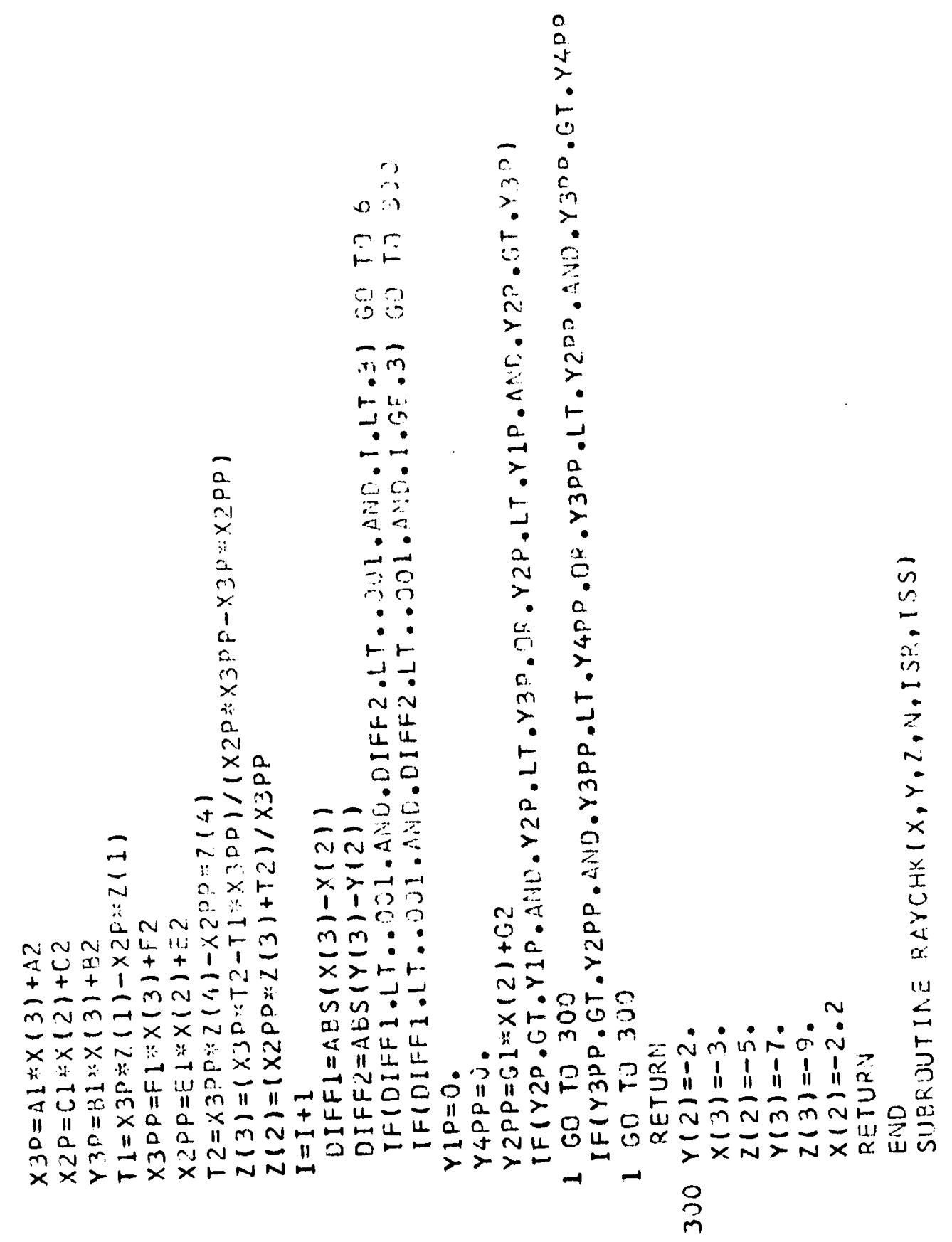



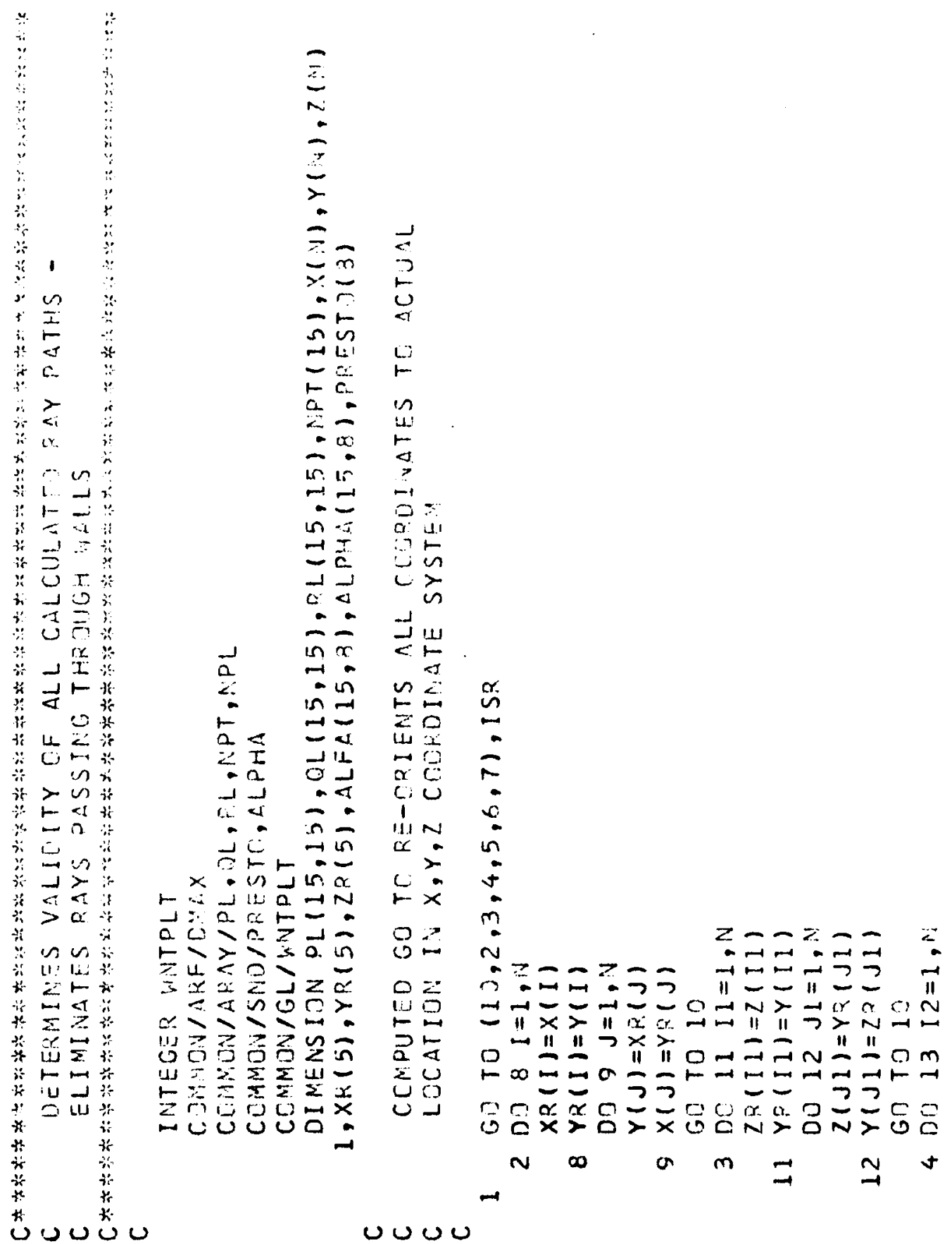

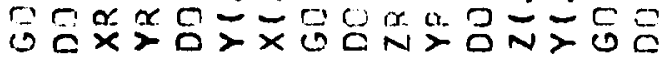
$N \quad \infty \quad m \quad n \quad r$ $\circlearrowleft \circlearrowleft \omega \omega$ 


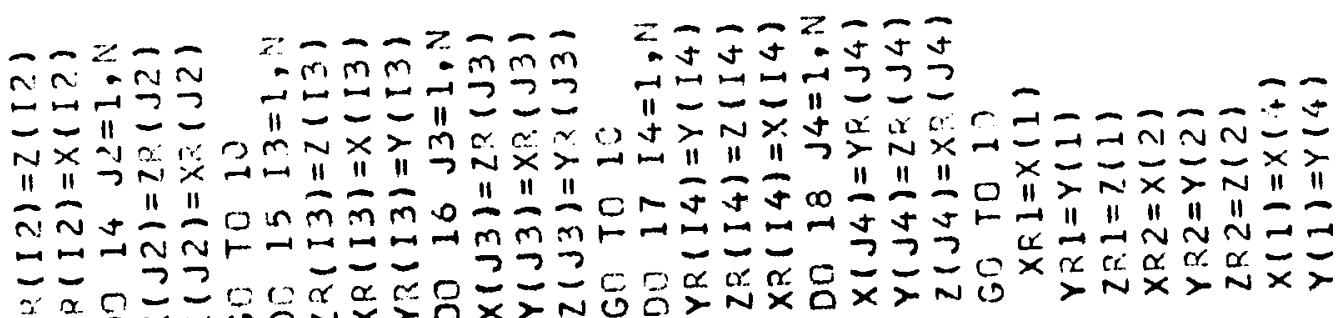

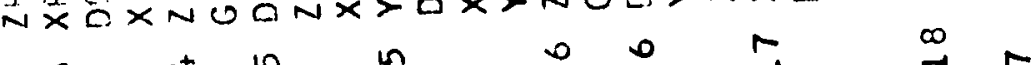

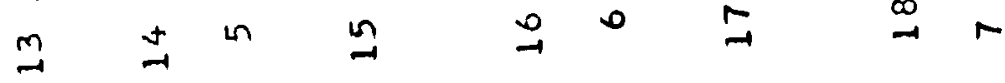




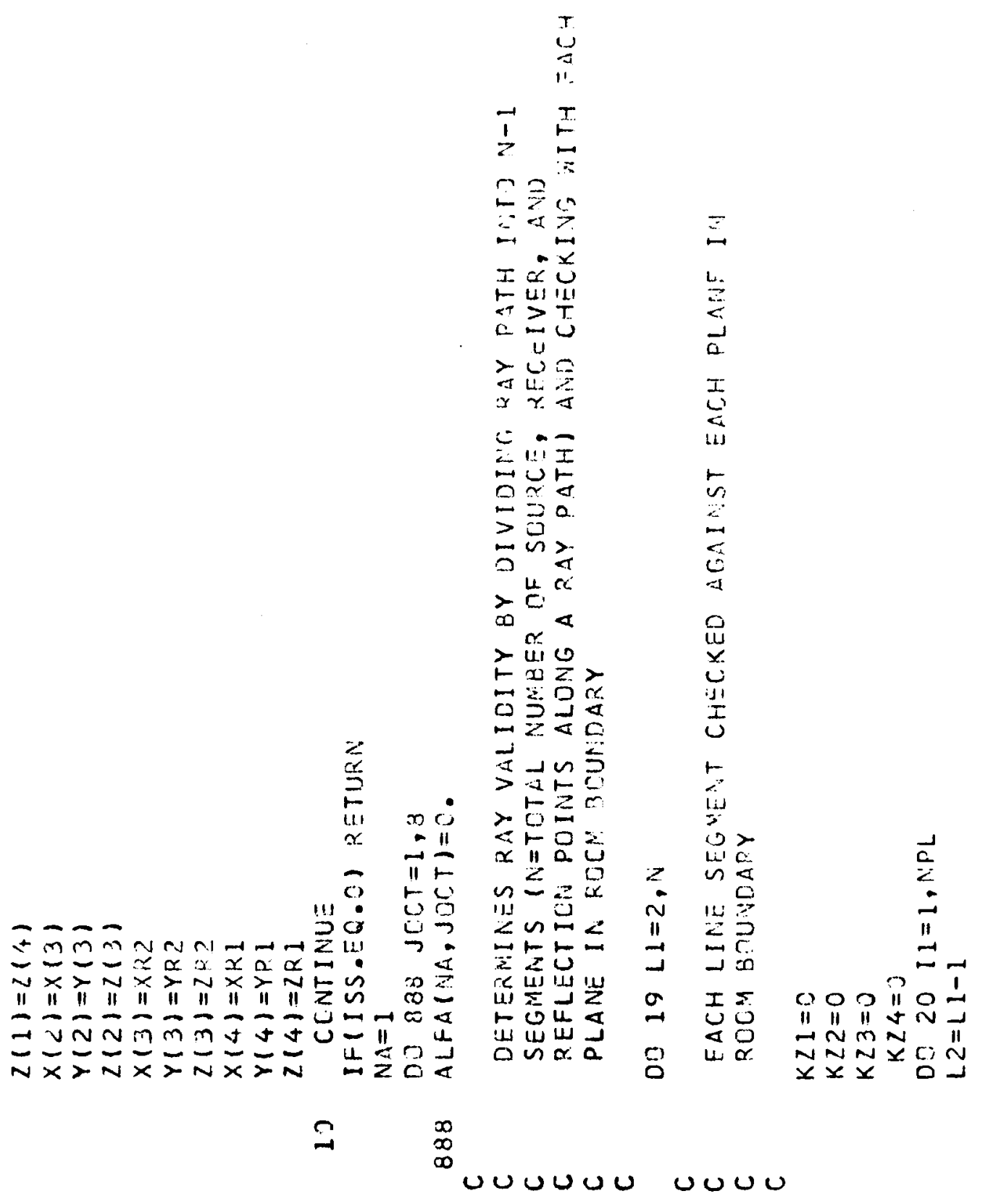




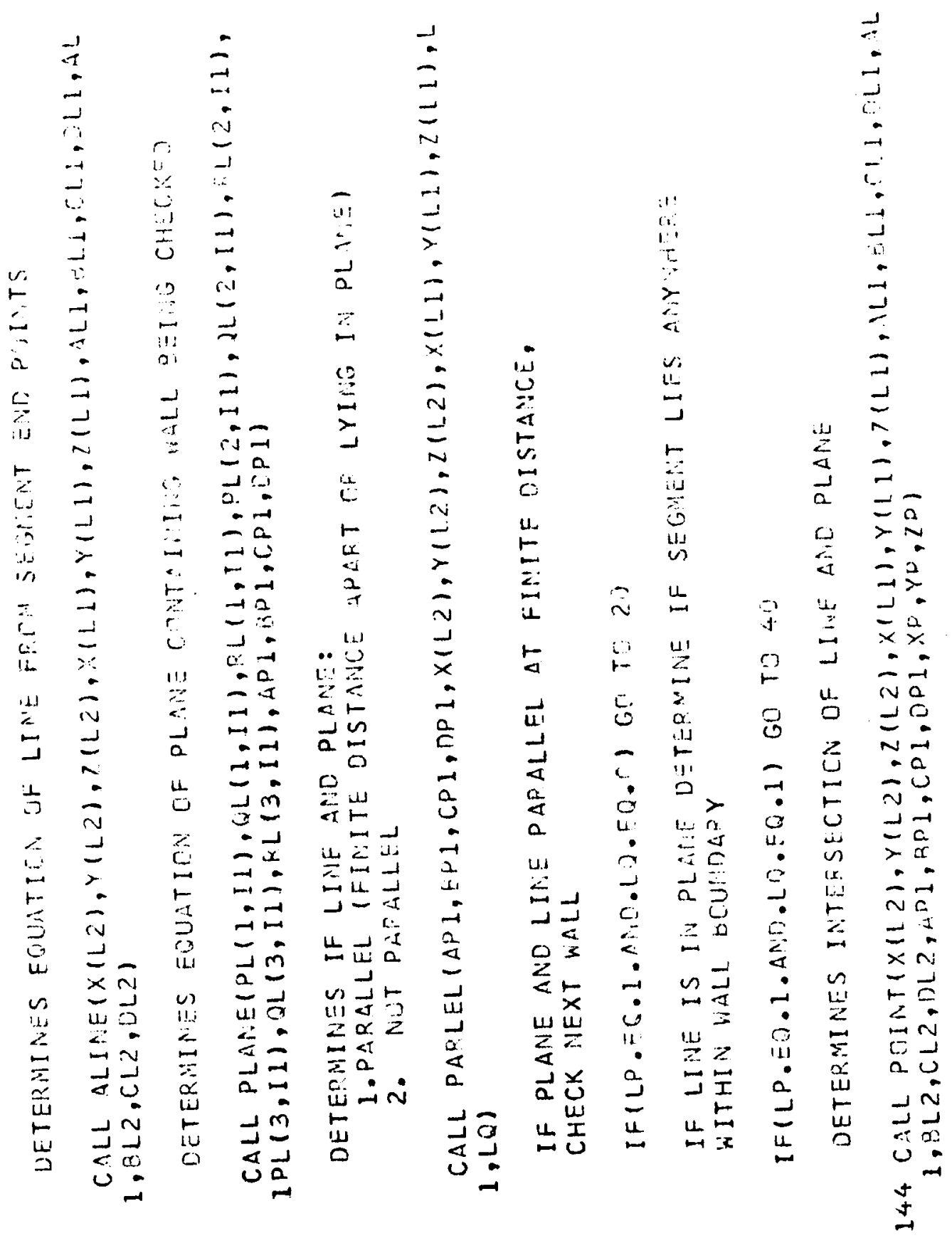

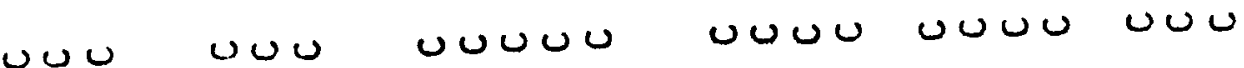




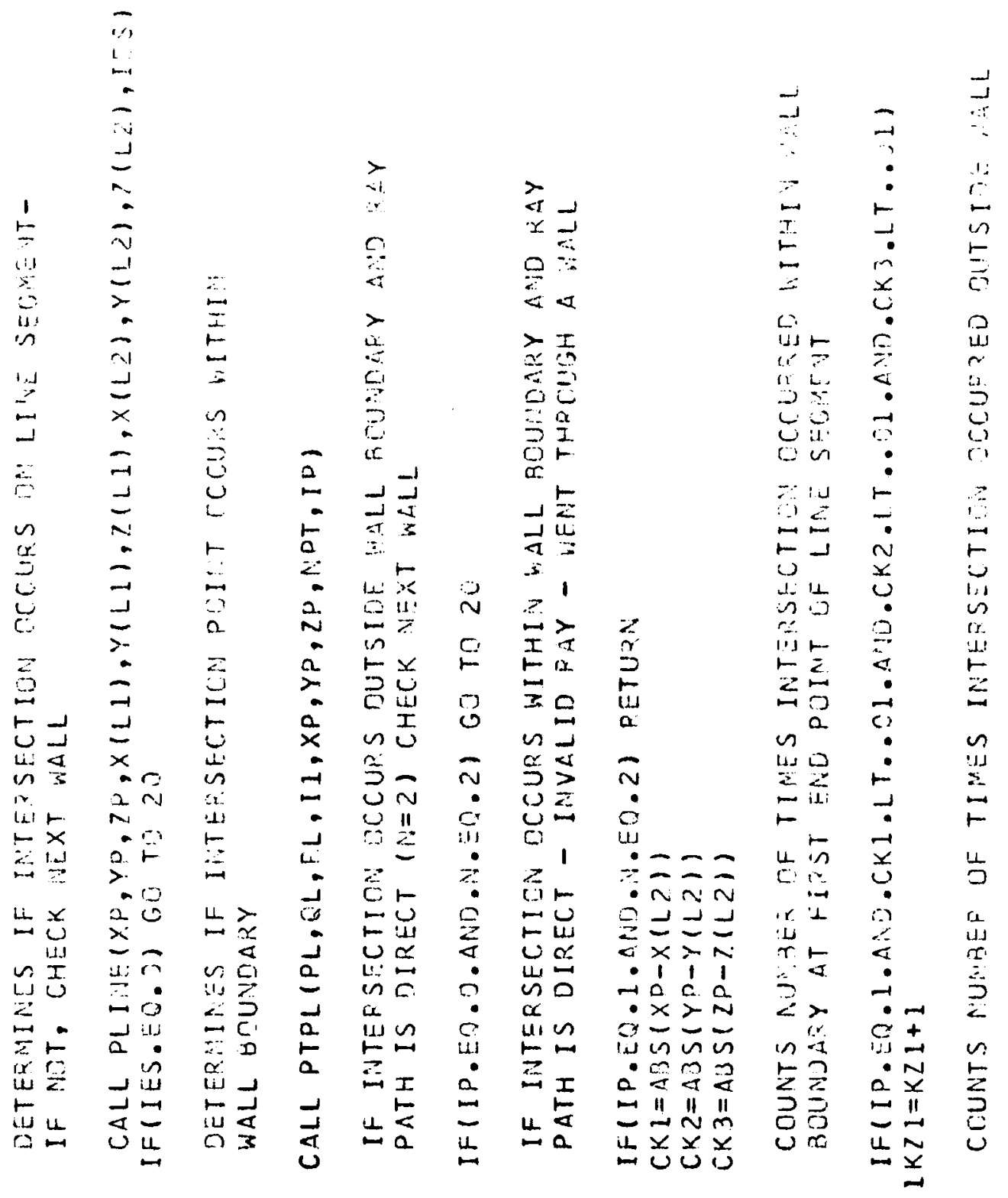

$m$

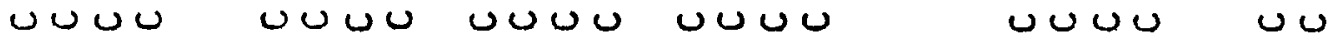




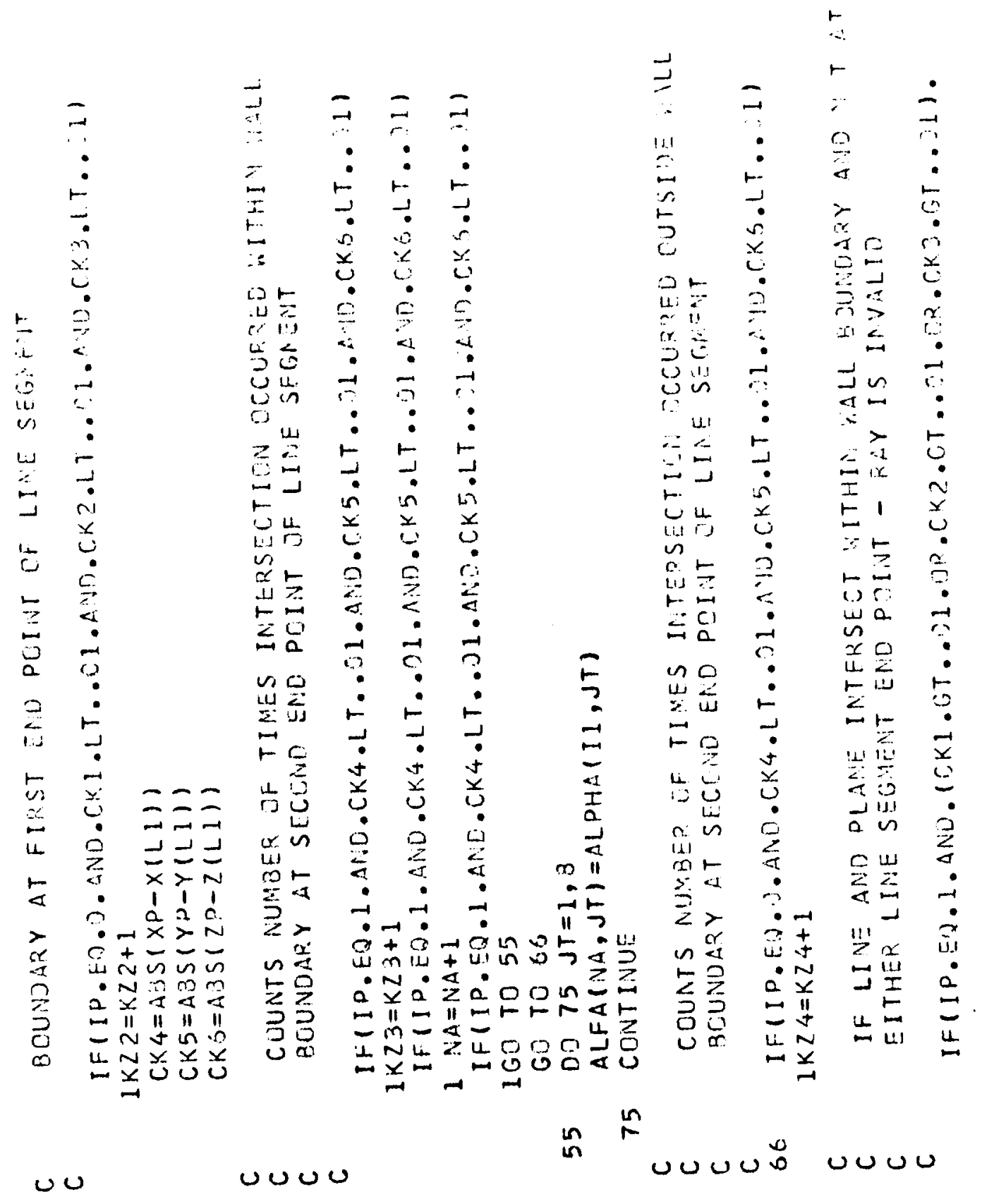




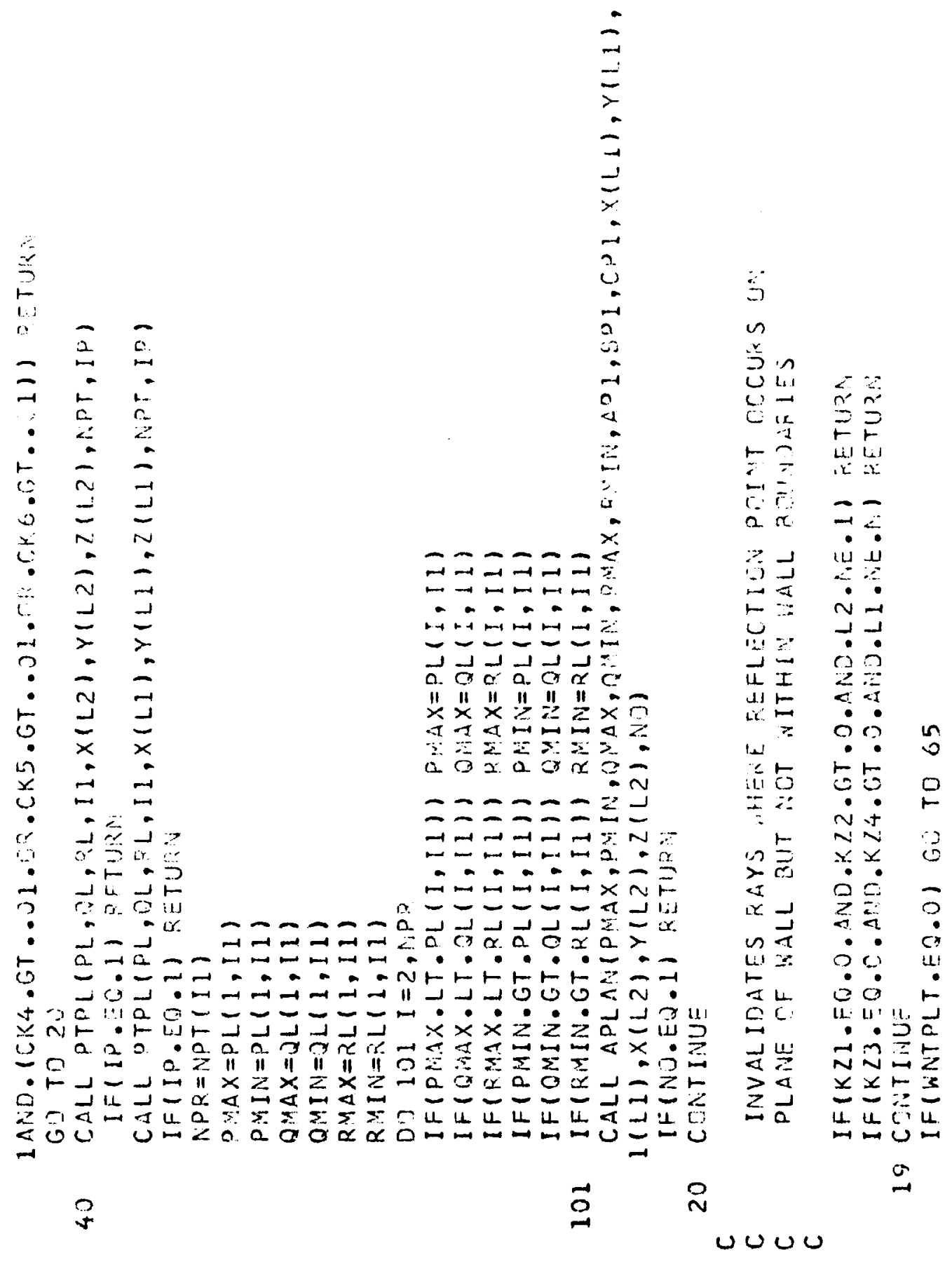




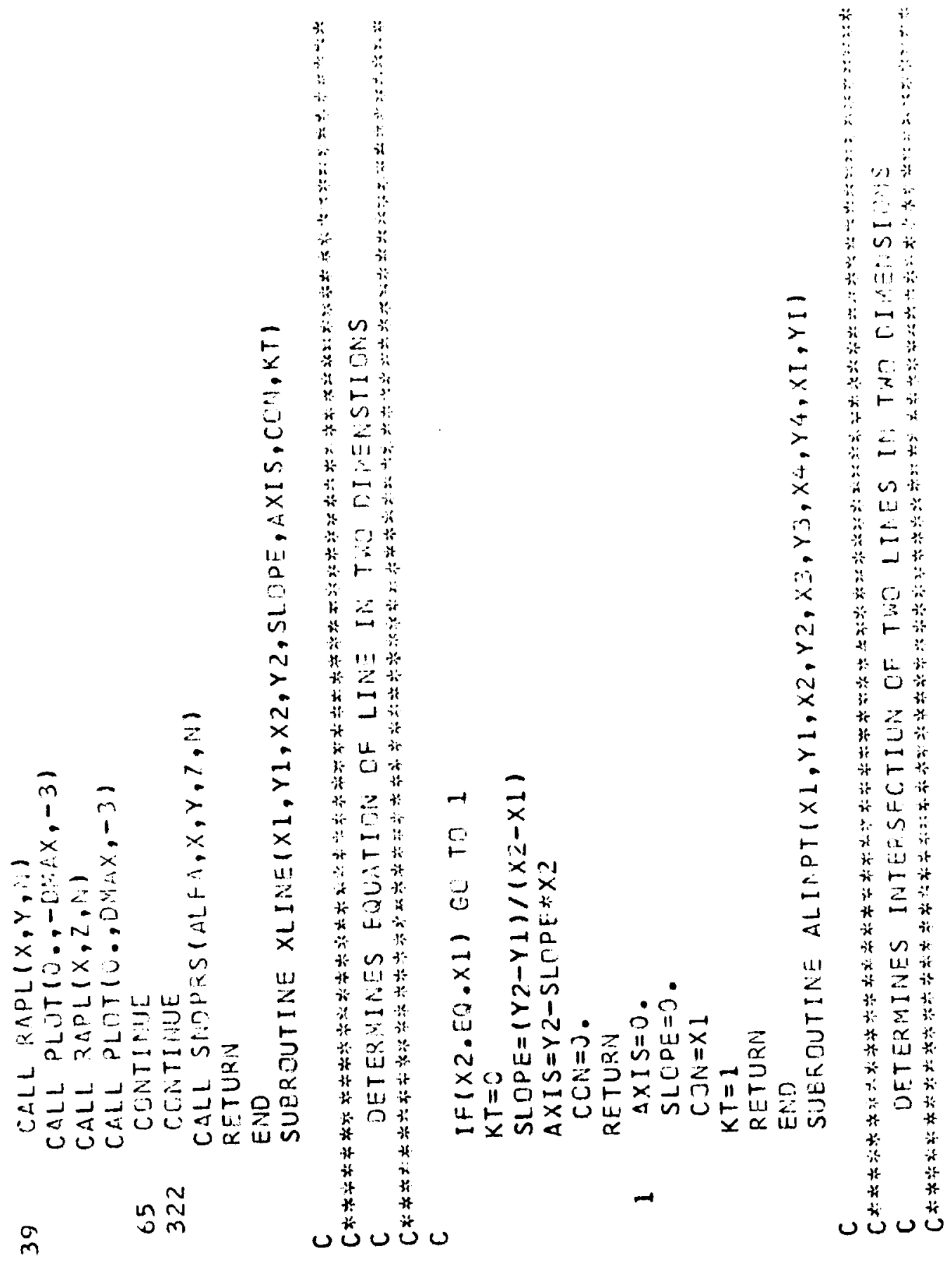



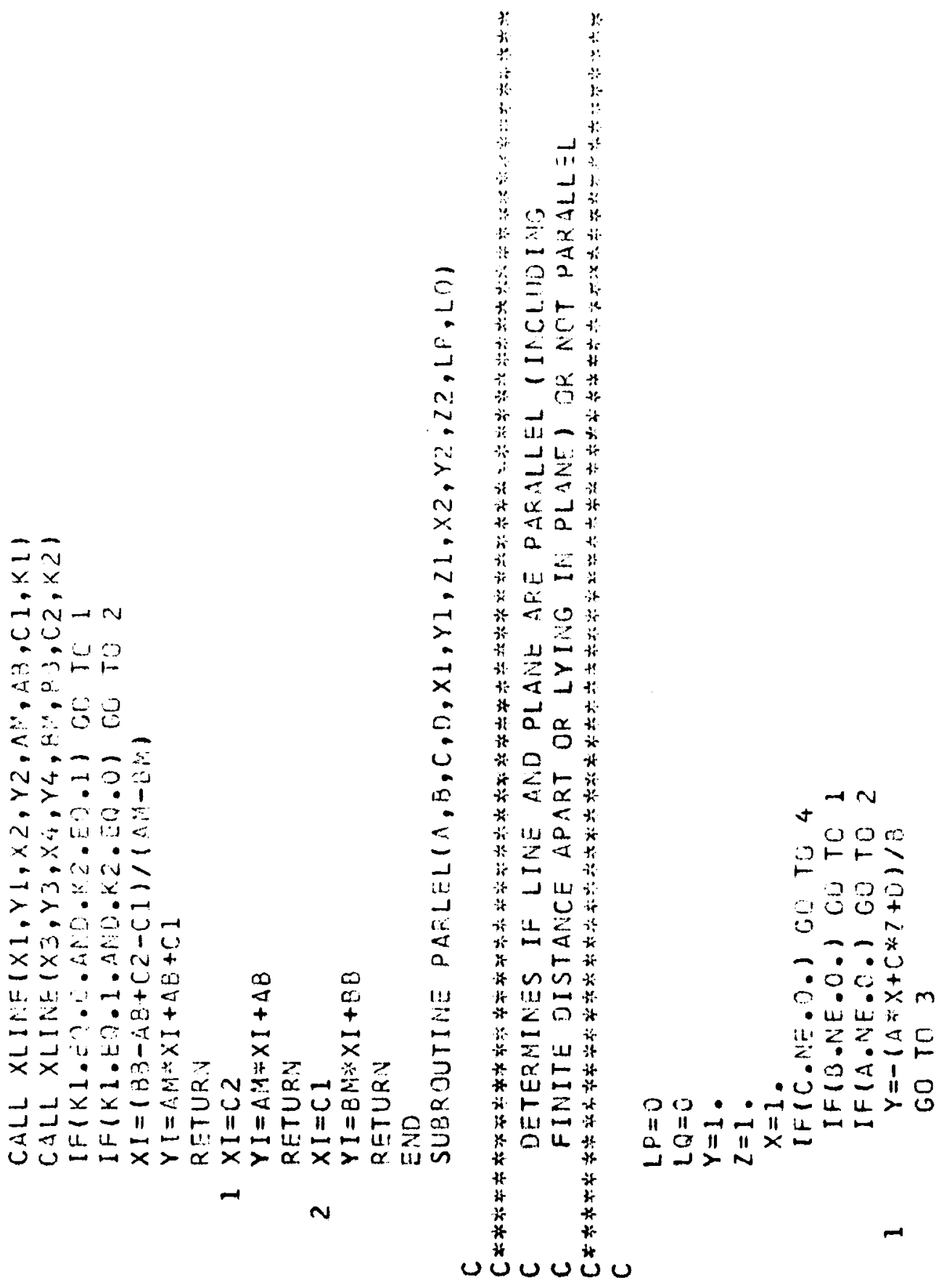


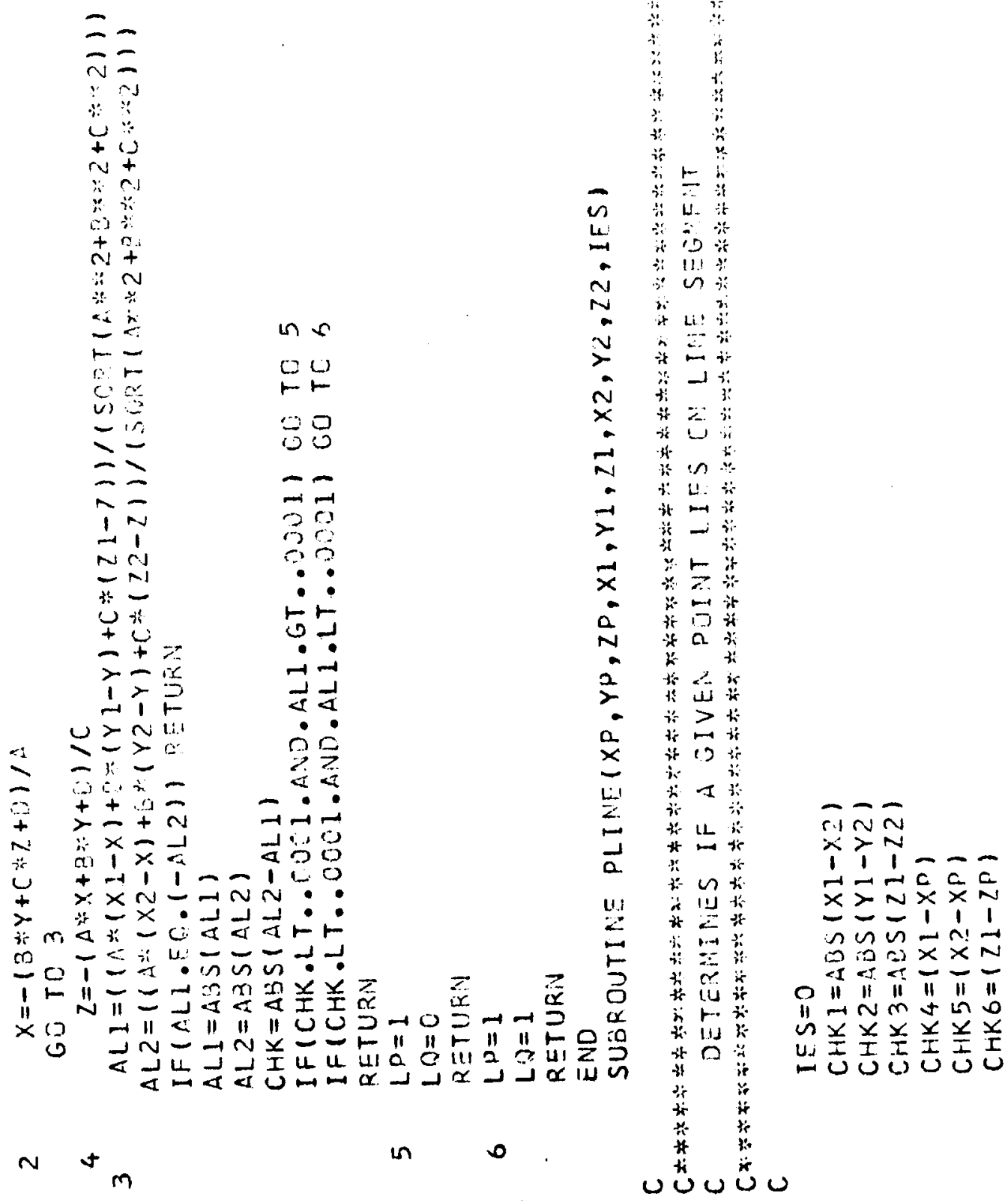




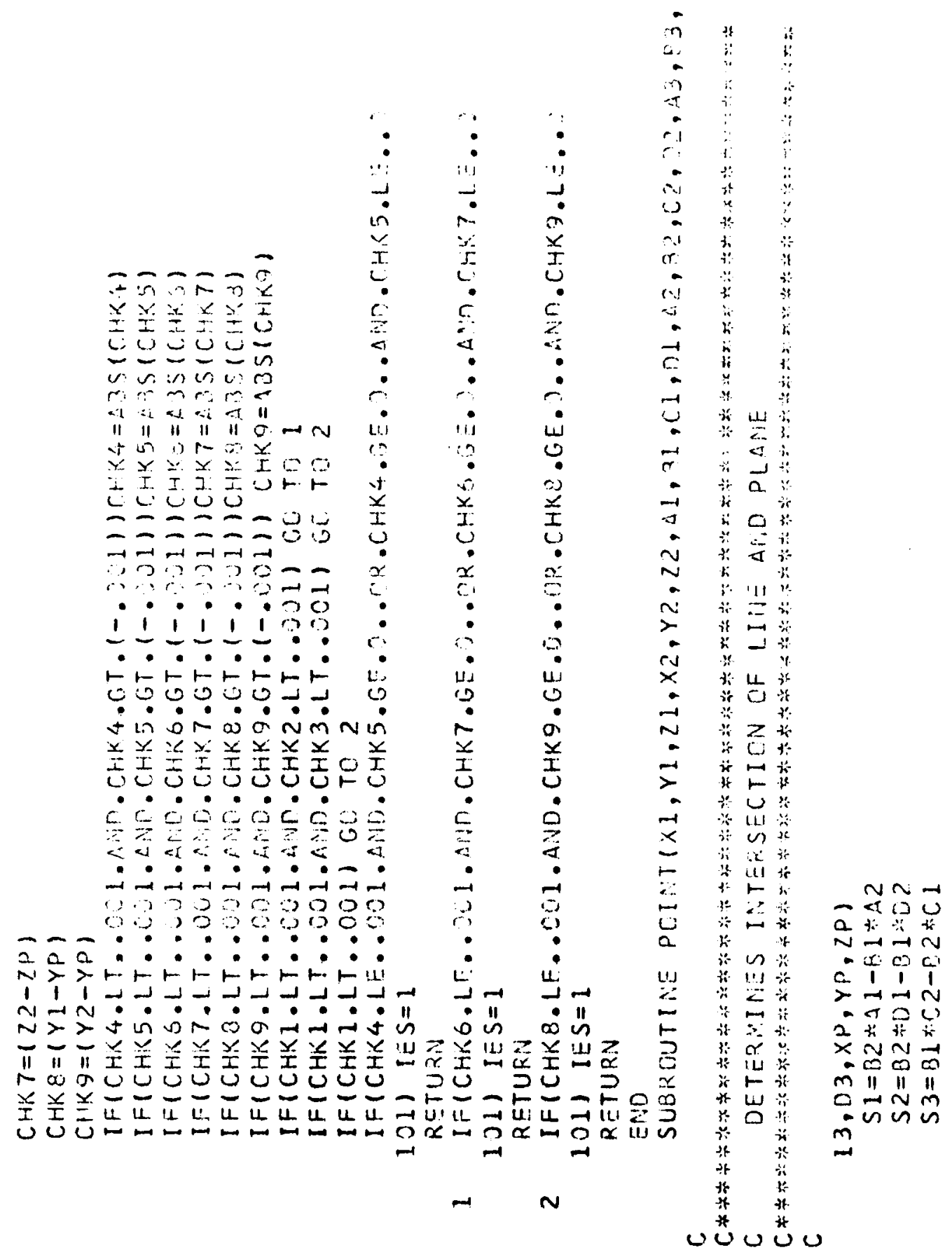




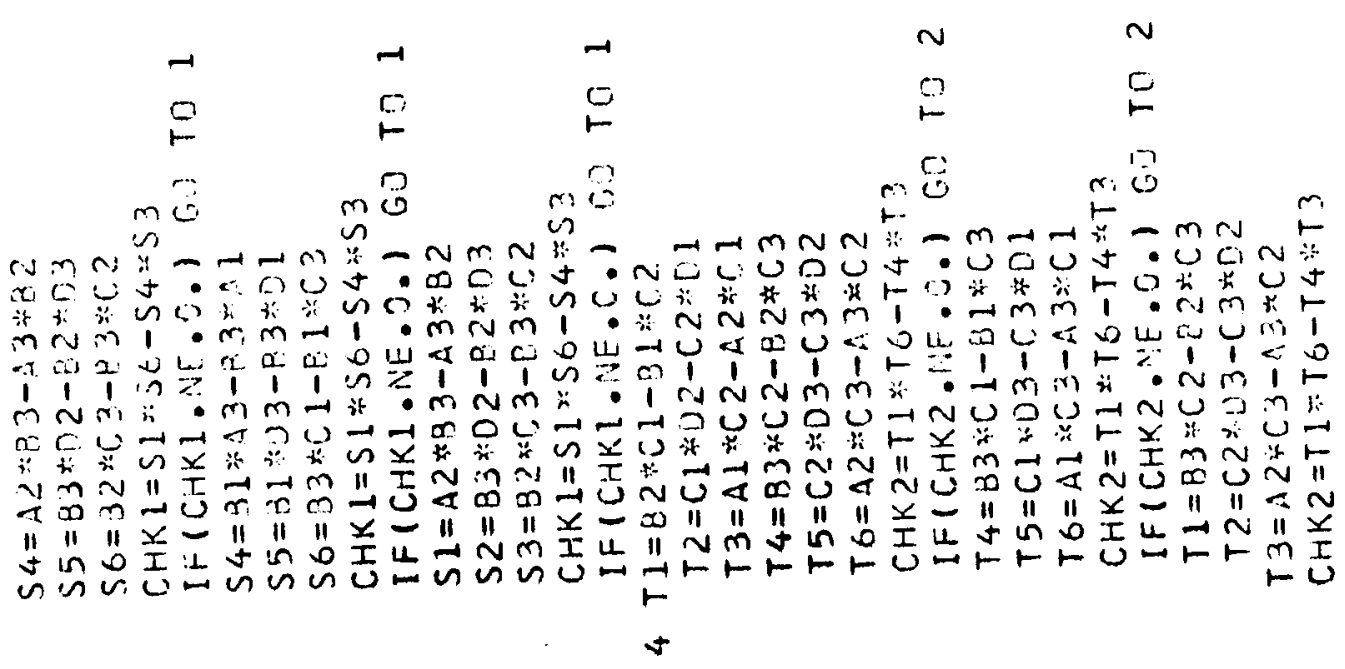




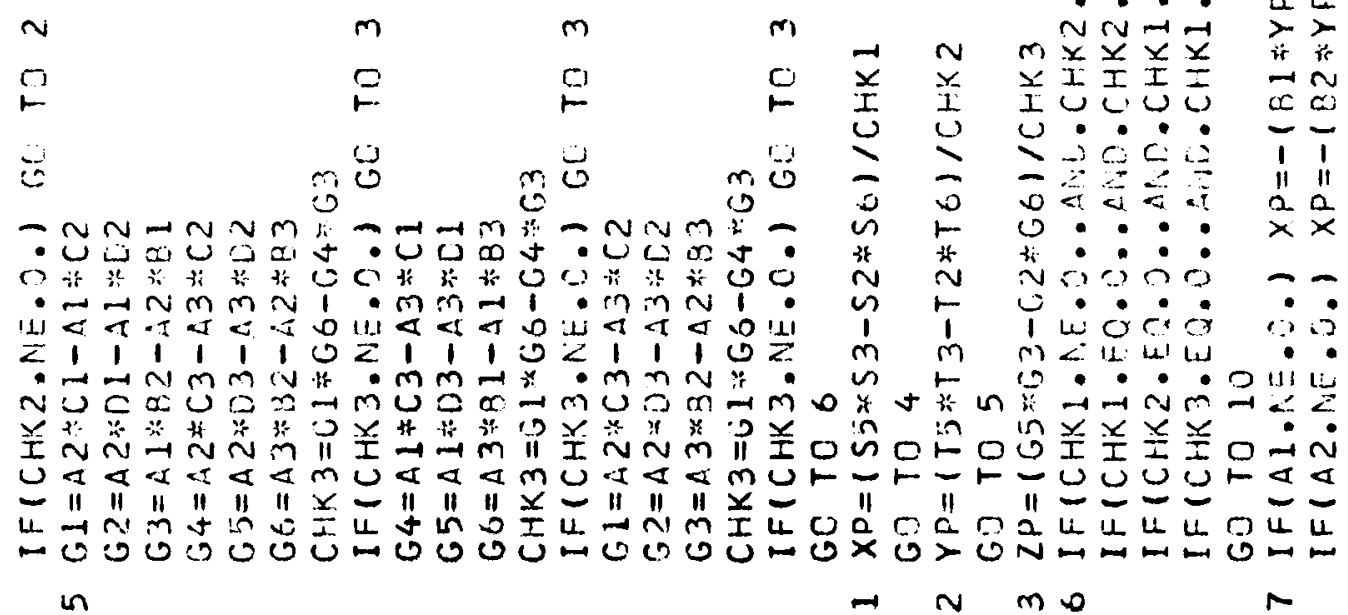




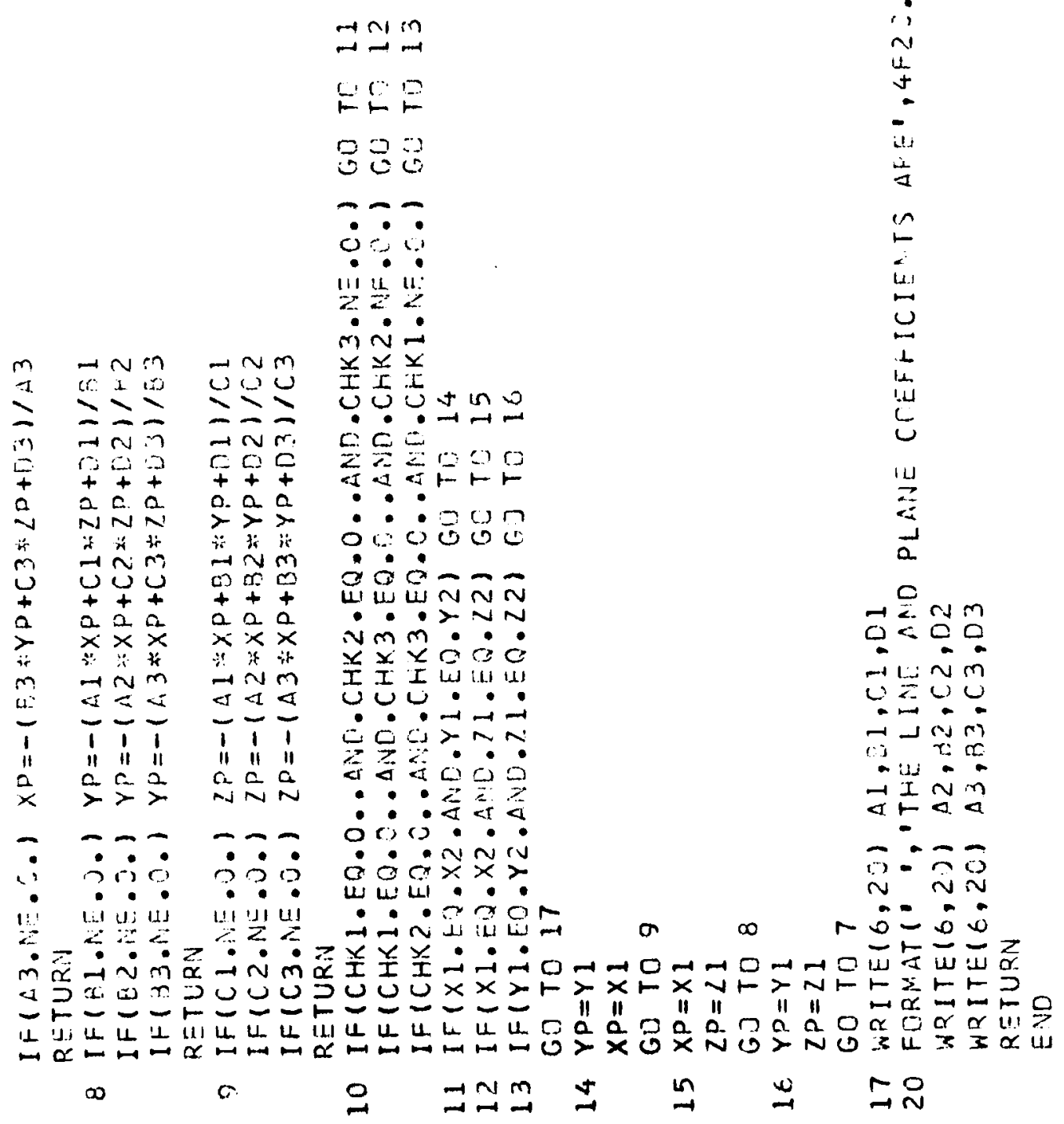


$N \cdot c$

$n \pi$

$\ln$

$\operatorname{arn}$

$n>$

in

$-x$

aid

IN

$>-$

$x^{2}$

$-\pi-1-1+1$

- $x<x \leqslant 0$

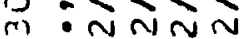

1. I

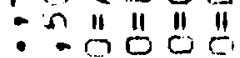

$\therefore: D$

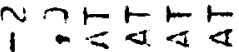

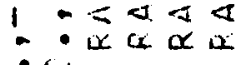

- $\cos$

-

$\sim N m+1$.

$x$ xxyt

)

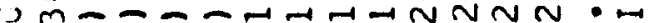

- - - - or a

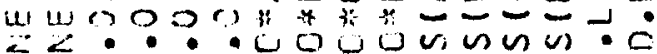

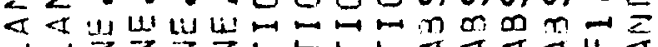

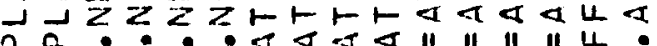

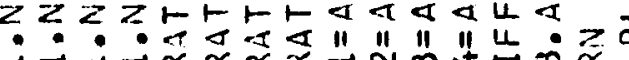

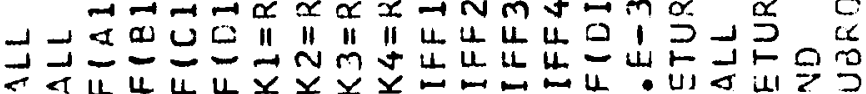
र U U.m-

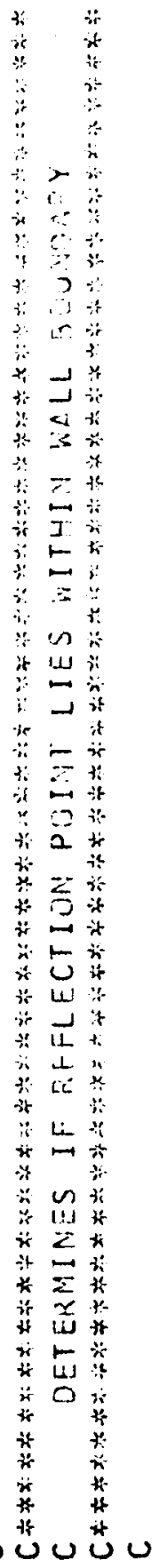




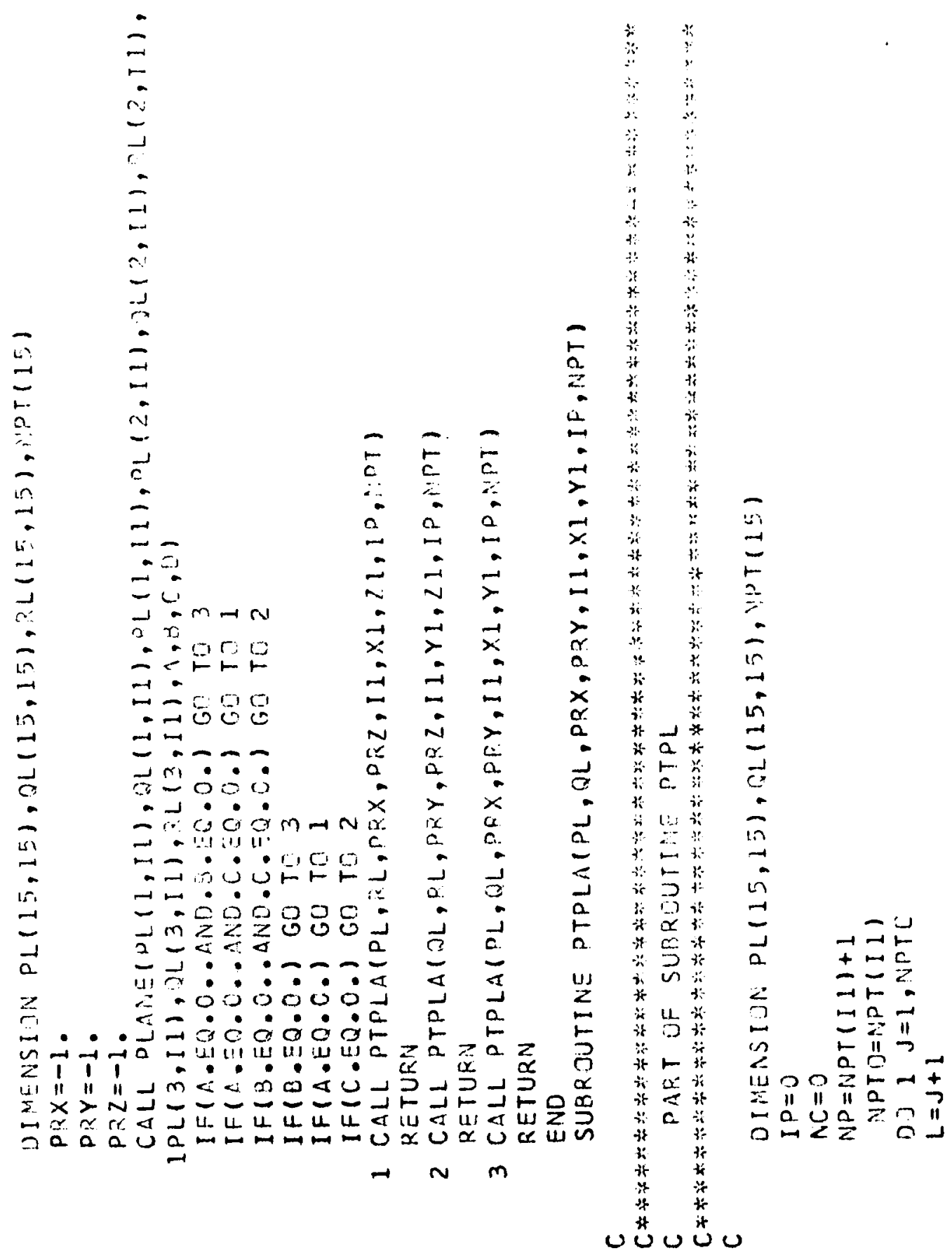



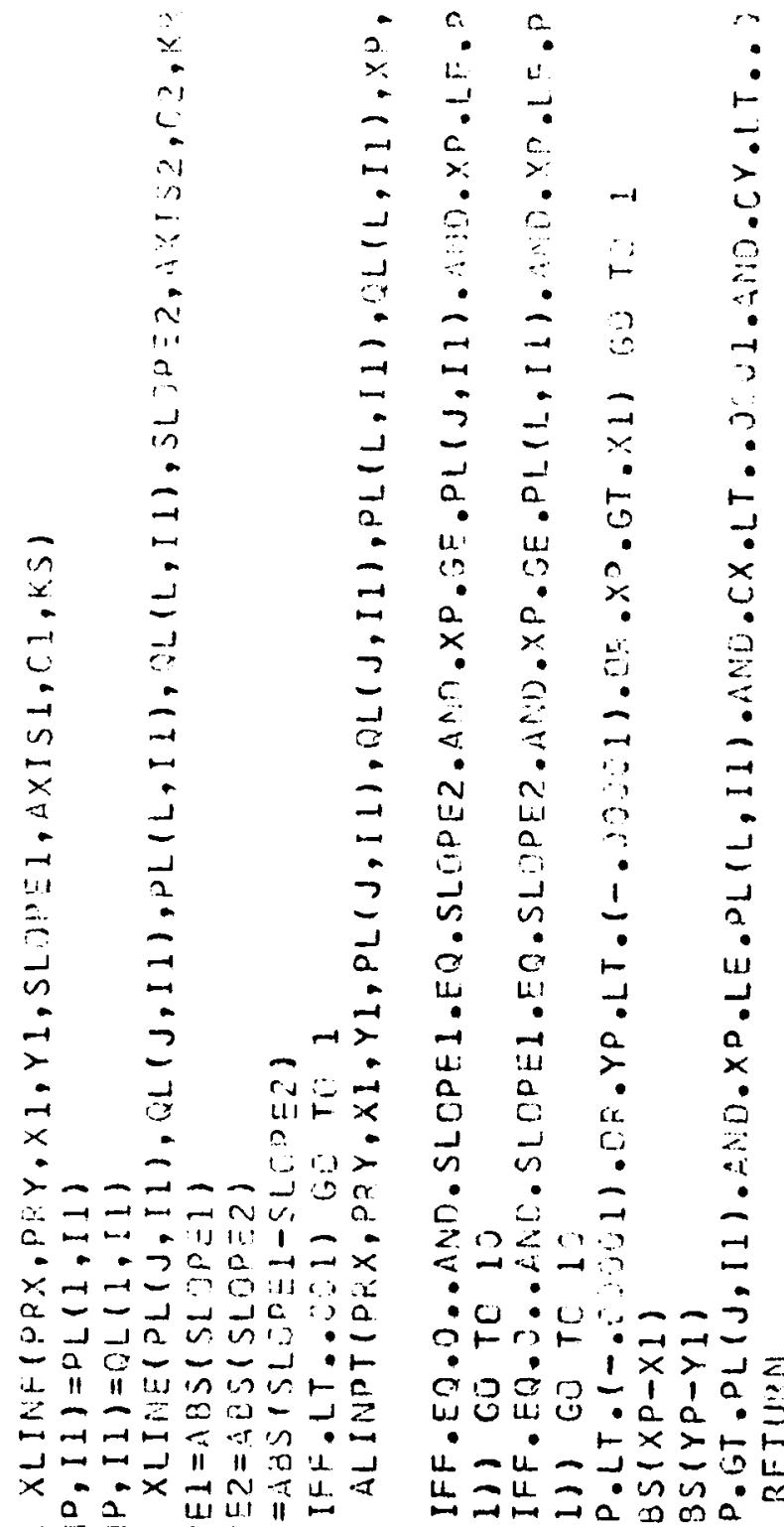

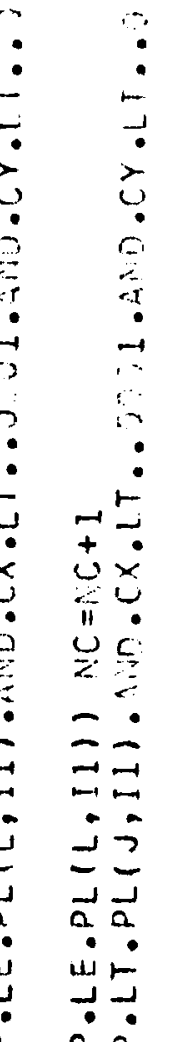

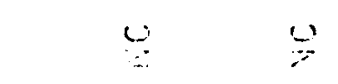

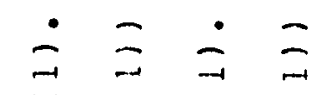

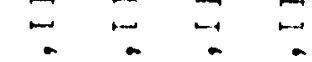

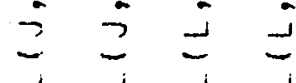

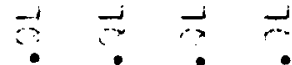

$a \stackrel{0}{x}$

$x \times$

ci

2

$\because \because \div 00$

$= \pm=-\because \vec{a}$

$m$

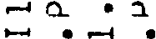

$\because-5 \% 0$

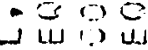

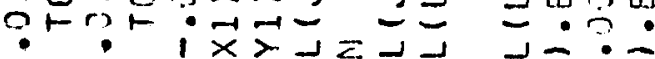

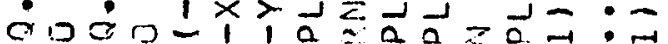

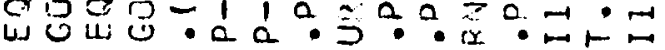

ú -

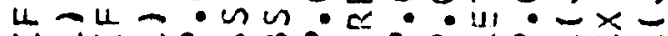

的

-

$\because-1$

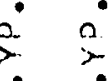

III Uा"

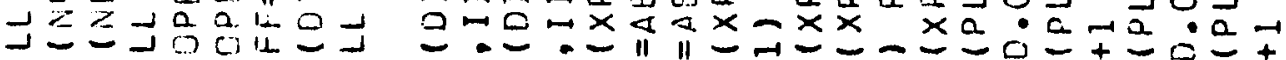

$x a \cdot a+\bar{a}=$

5
2
$\square$
$\frac{1}{0}$
0

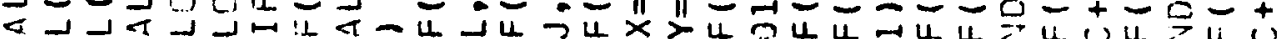

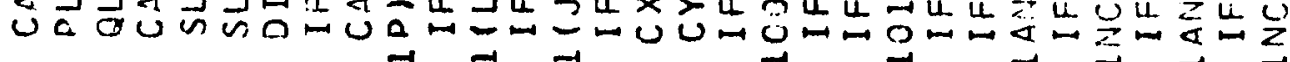




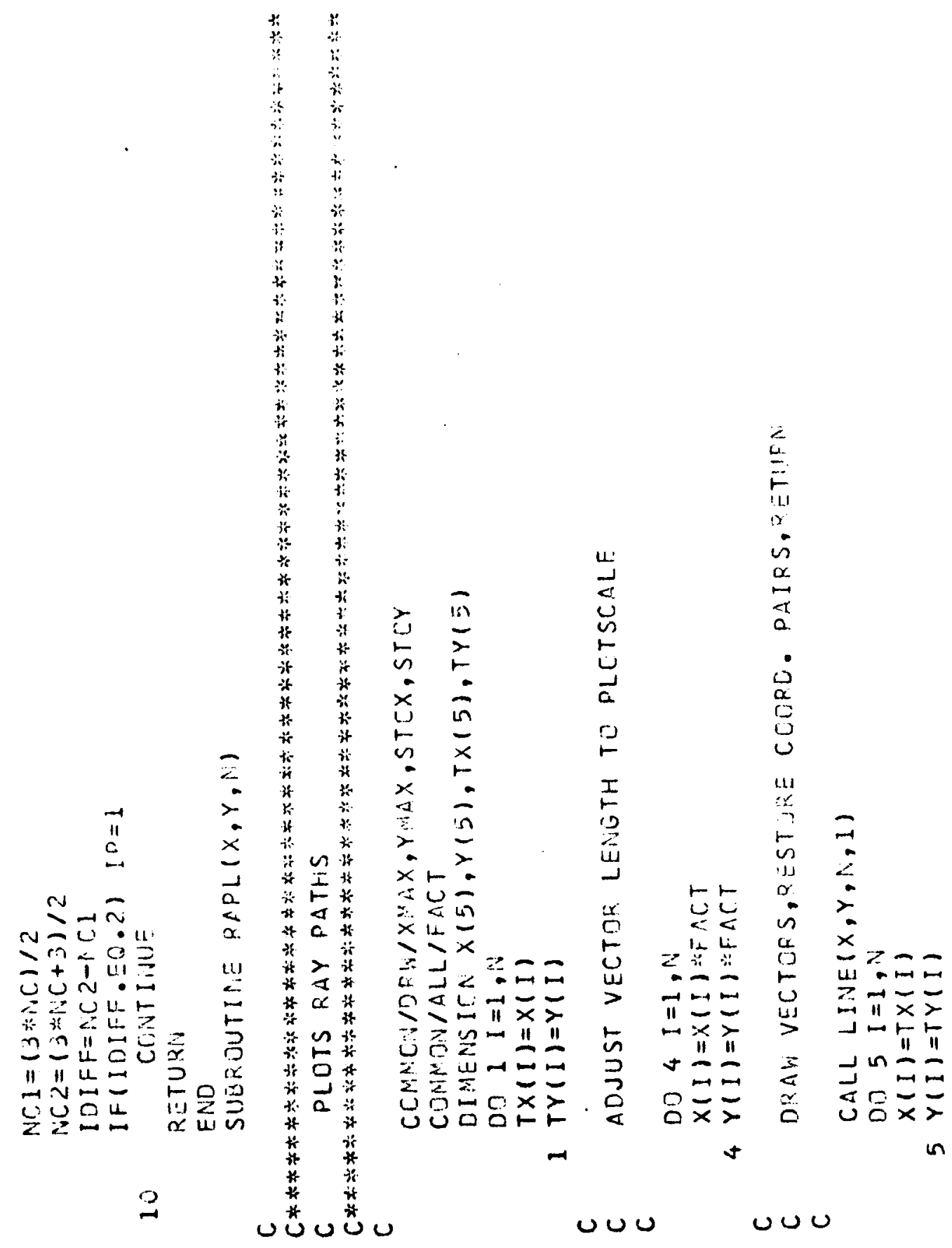




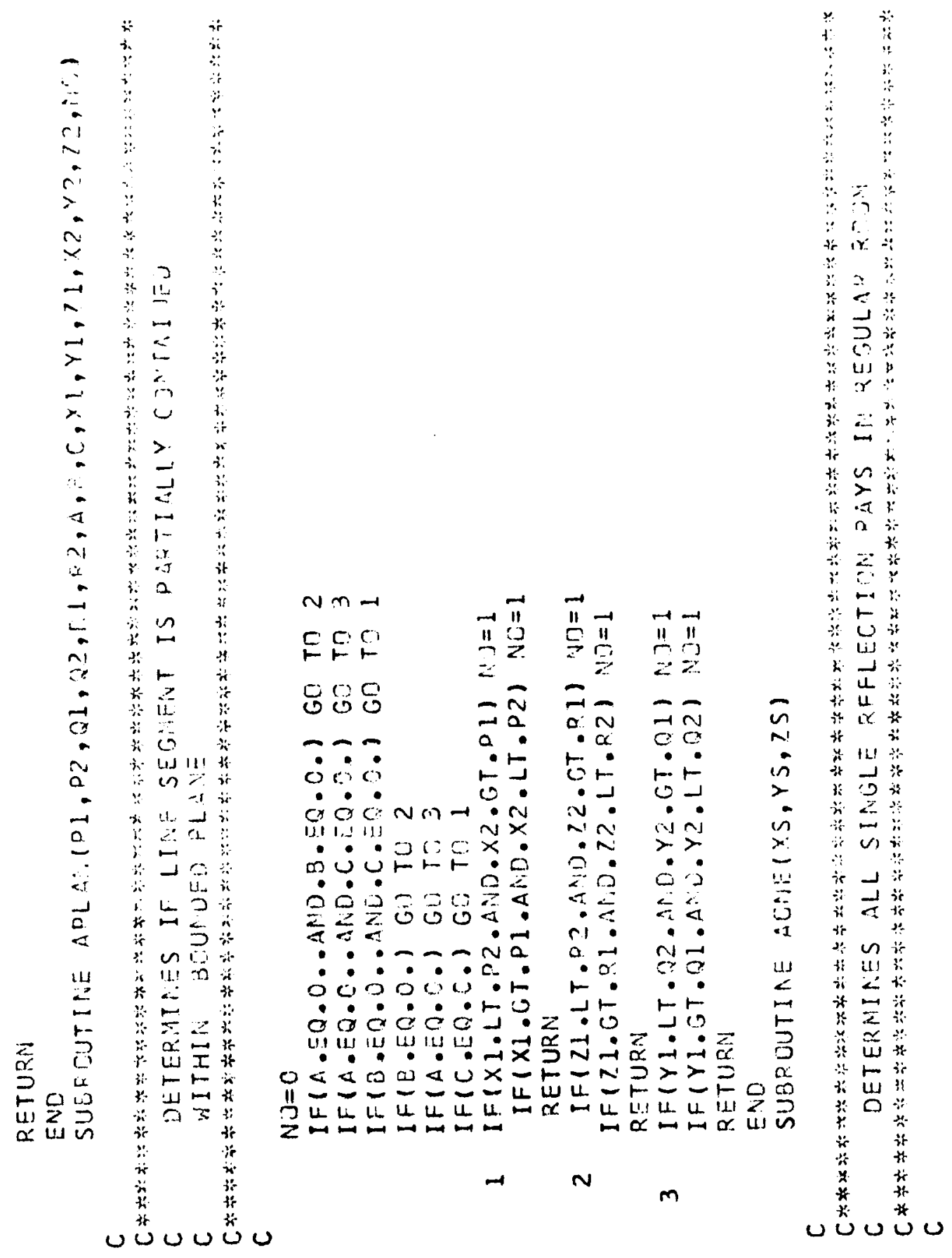




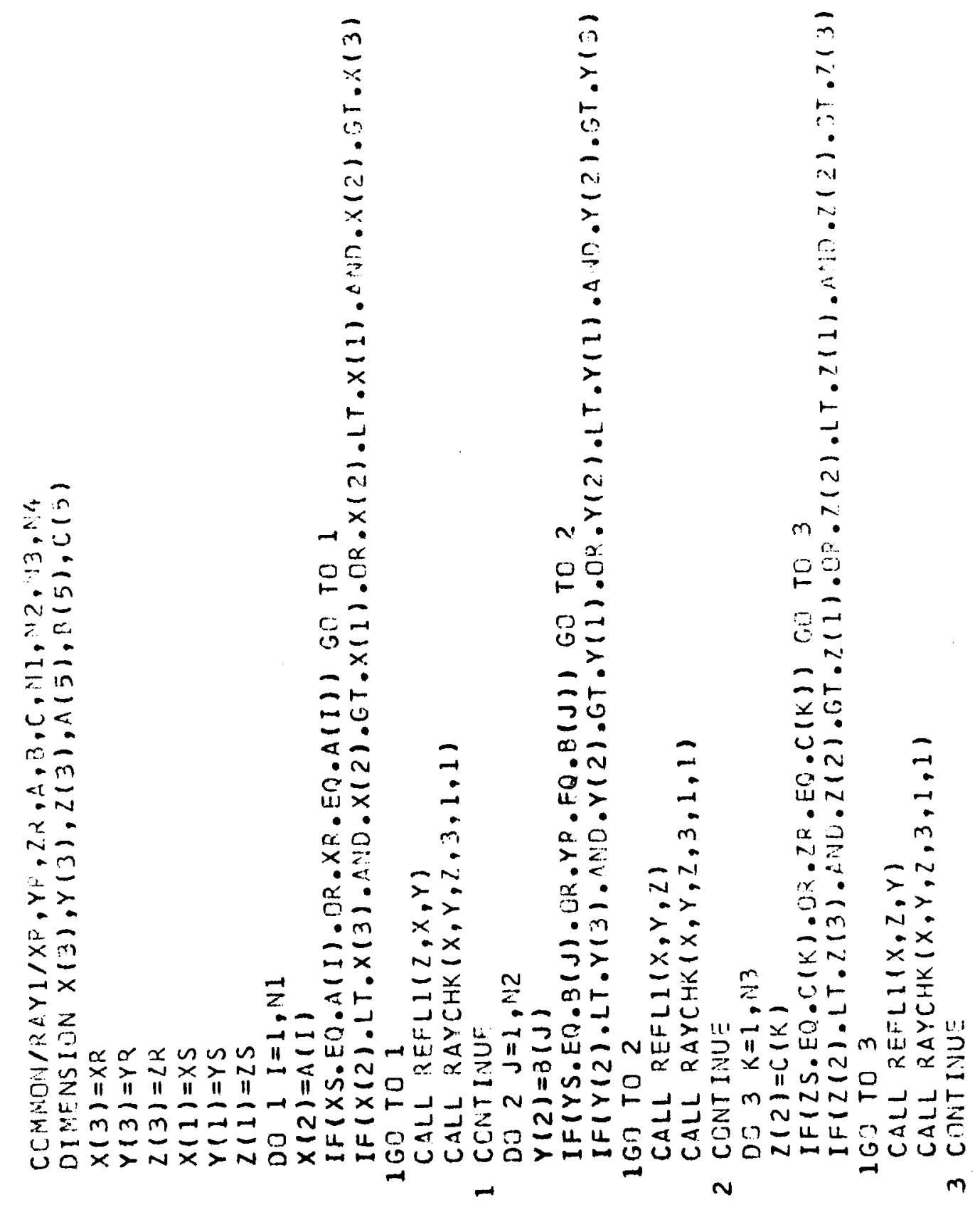



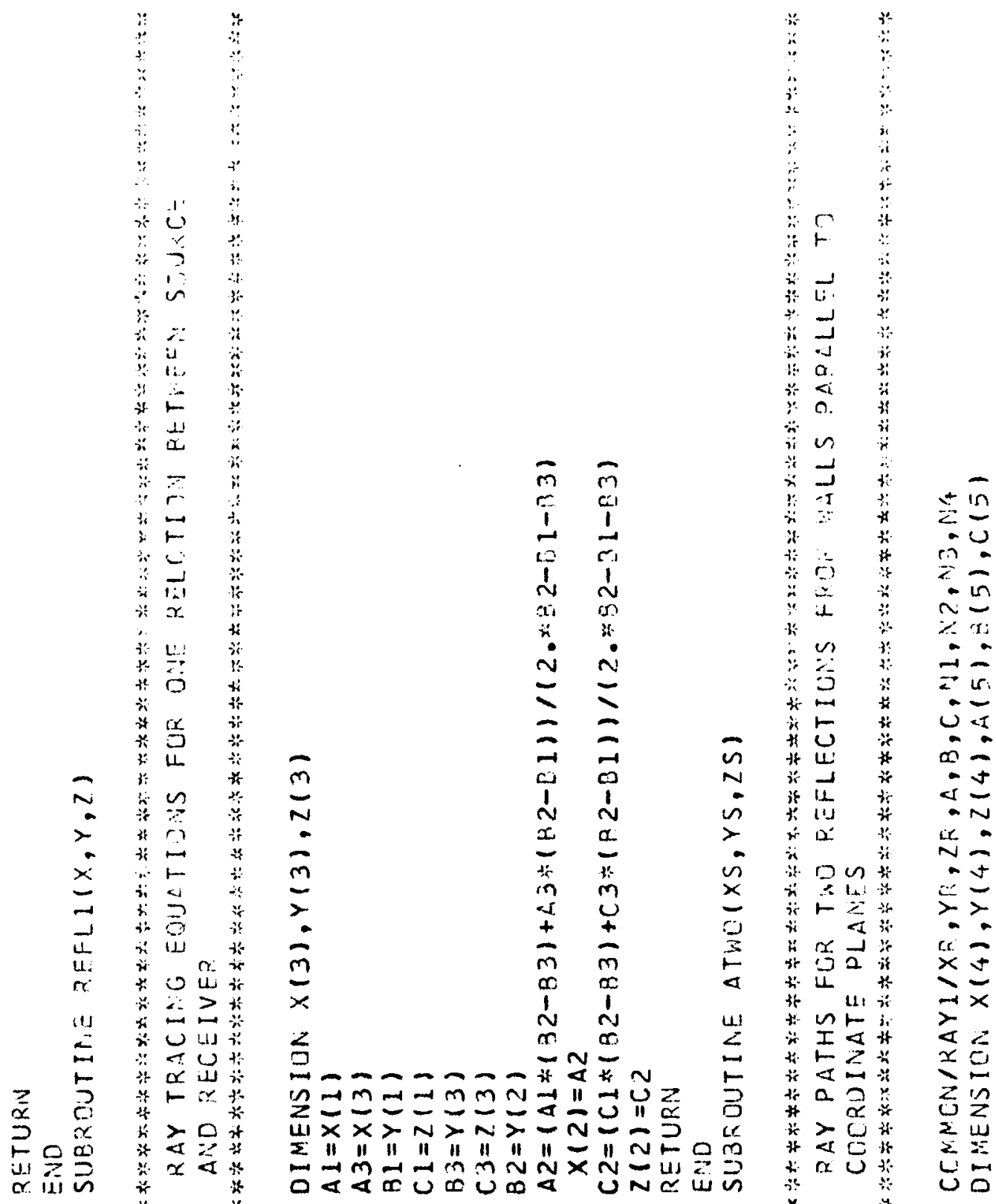


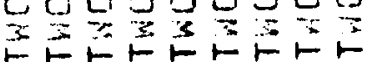

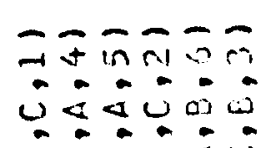

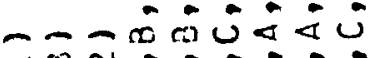

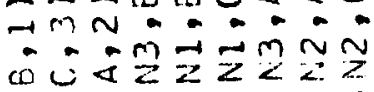

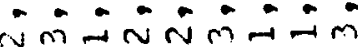

$N m \rightarrow N N m \%$

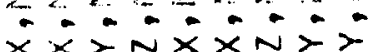

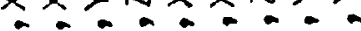

$\therefore \dot{*} ;>\hat{x} \dot{x} \dot{x}$

$>n \times>>-n \times m$ $\hat{N} \sim \hat{N} \vec{N}>\vec{N} \dot{x}$

$=-2=-2=$

COCOOOOOC

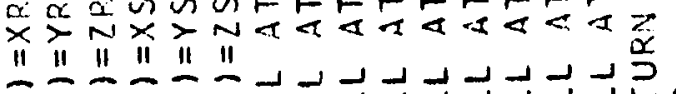

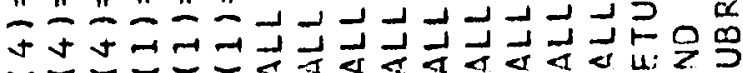

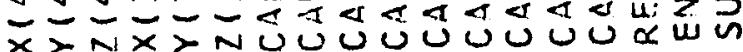

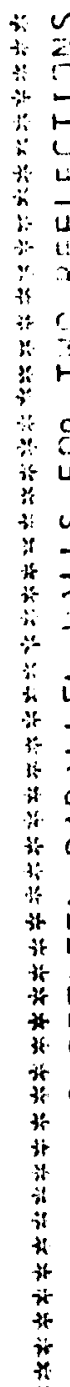

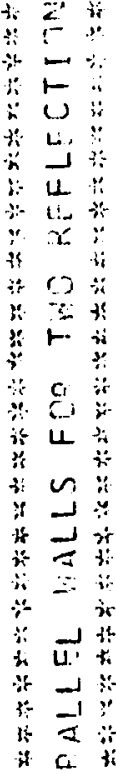$$
\text { \#* a }
$$$$
\text { if a }
$$

1)

it 4 )

it L:I t:

से 5

* 山ा

3.

*

I

if $F$

it 5

w 0

70 c

H. $>$

it 2 I $*$

$\ddot{x}$ or

if

ix w

II 11 it

if 2 if

it $\because \ddot{x}$

艾获

मे

it

* 口

if

in

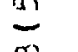

$\stackrel{0}{-1}$

$\pm$

N

$\pm$

$=$

$\pm$

$\bar{x}$

$\cong$

$\frac{n}{1}$

$\angle-\cdots-O N-N$

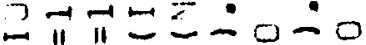

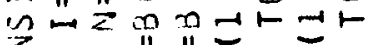
莎

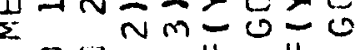

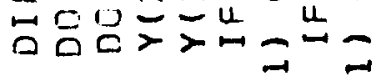




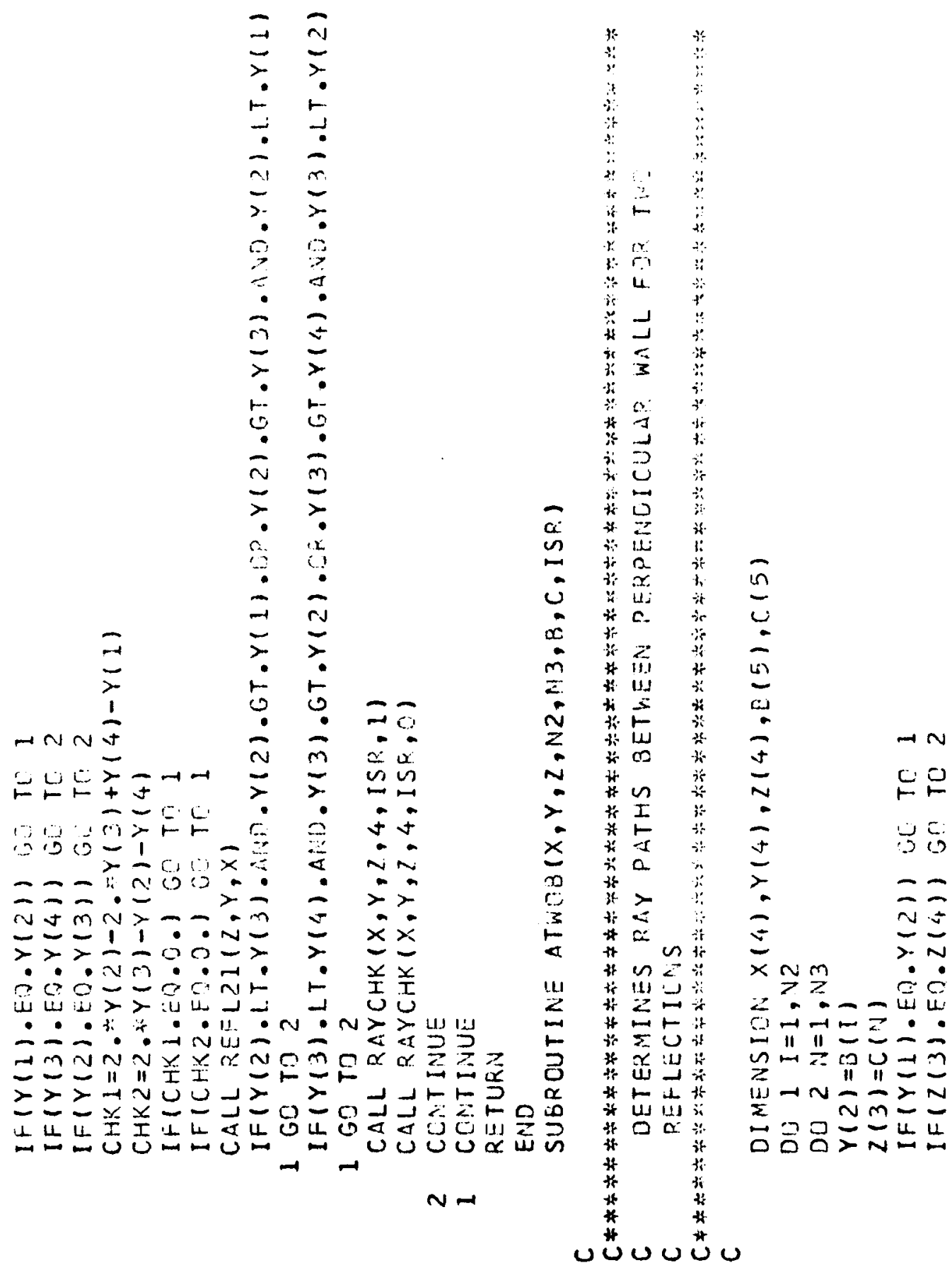




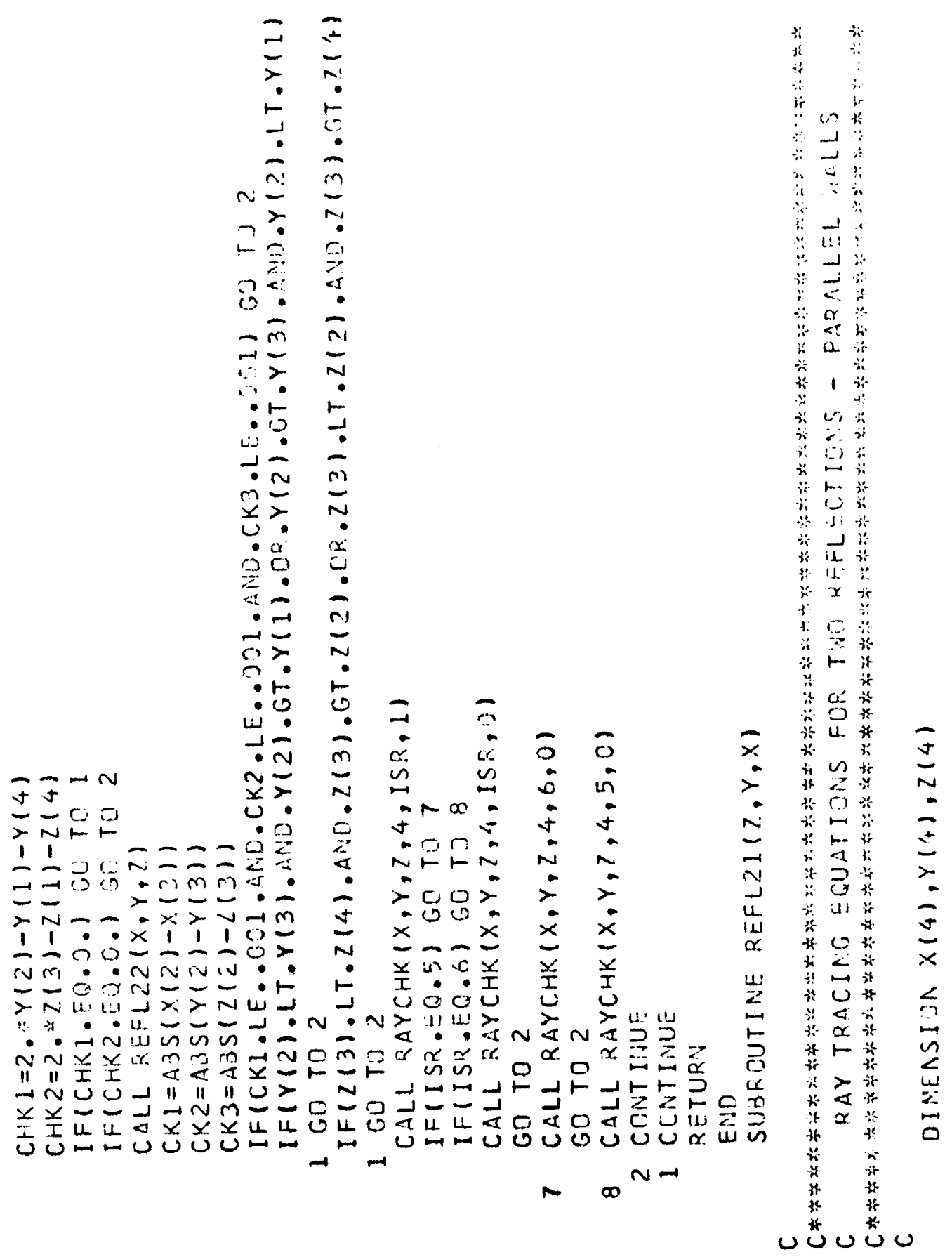




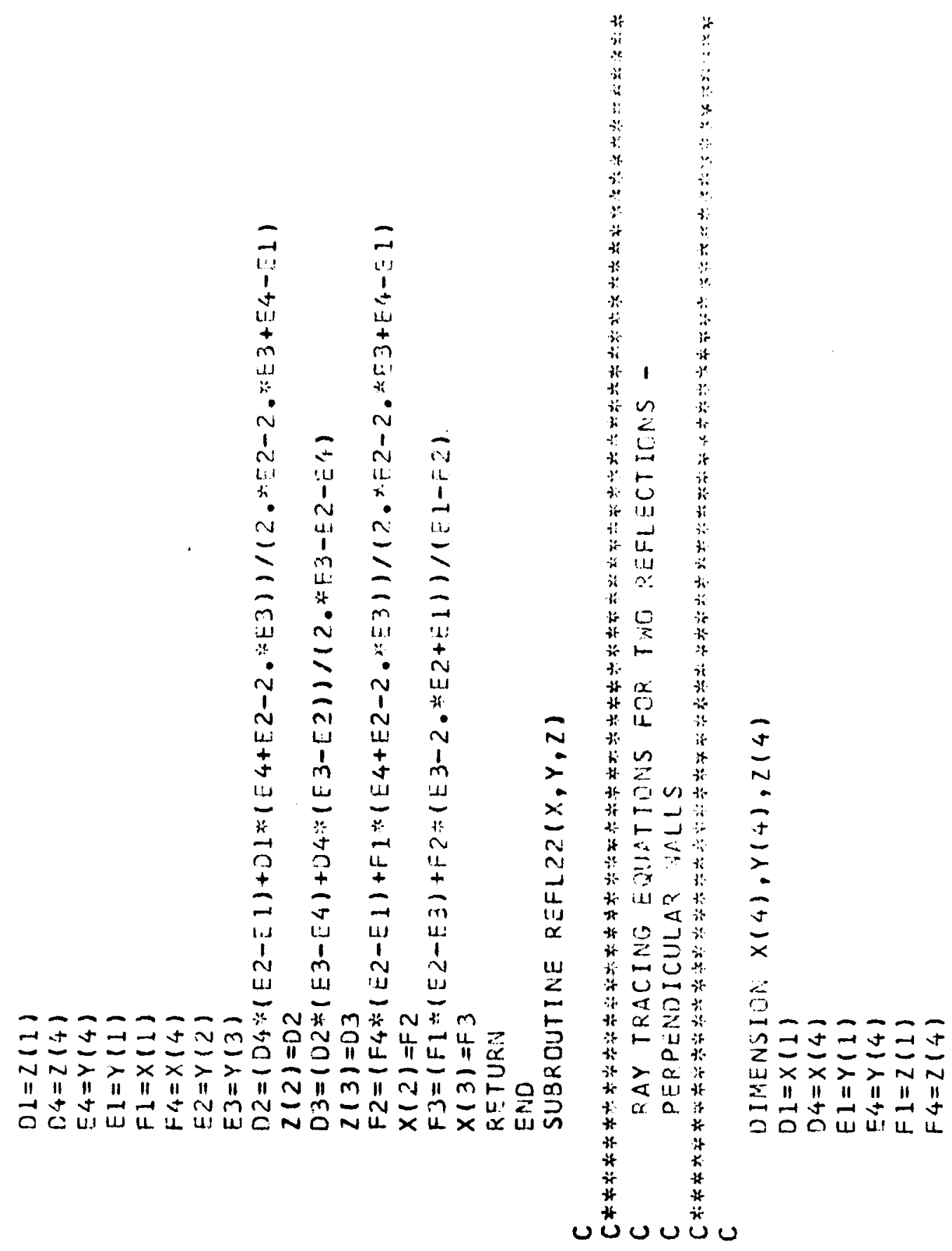




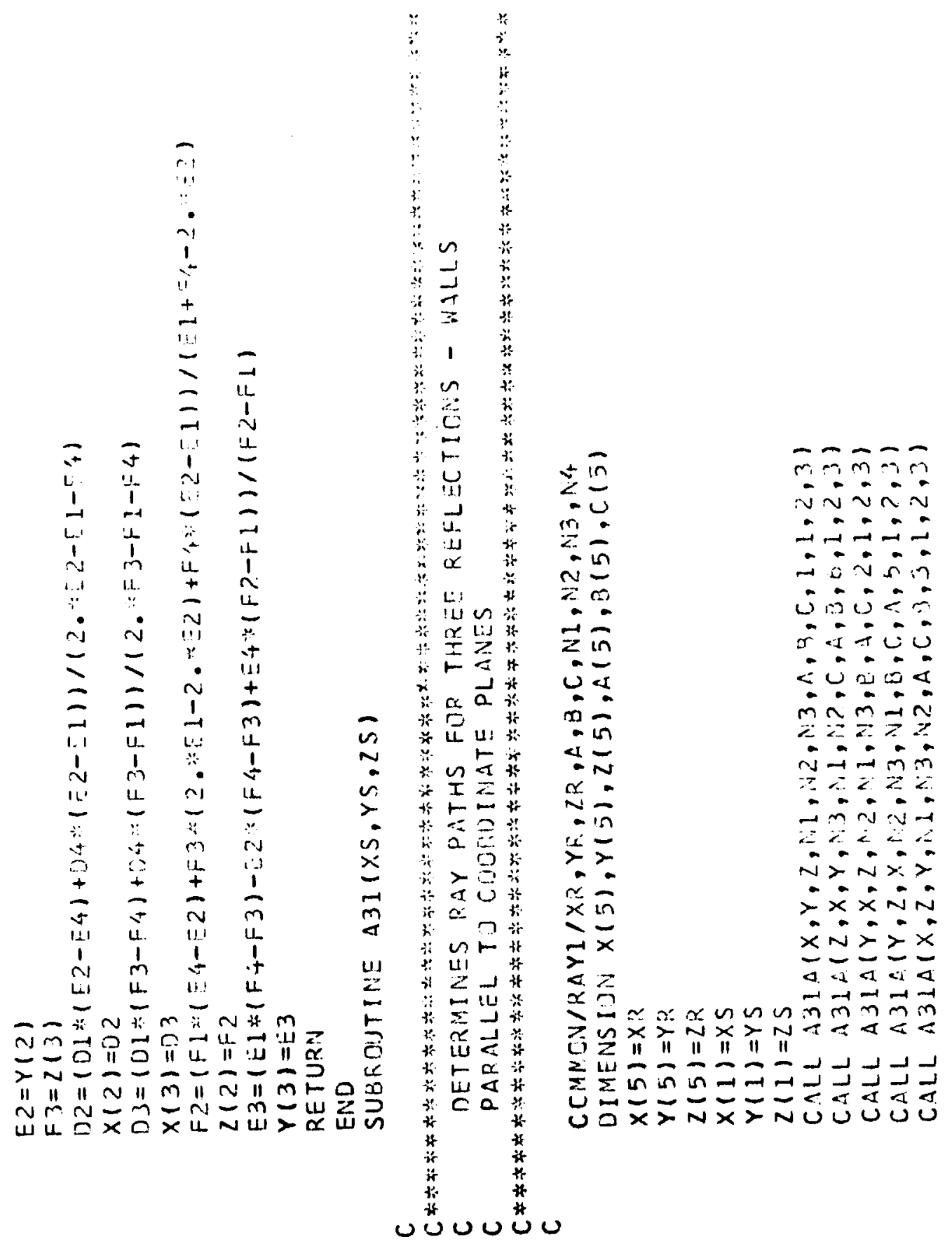




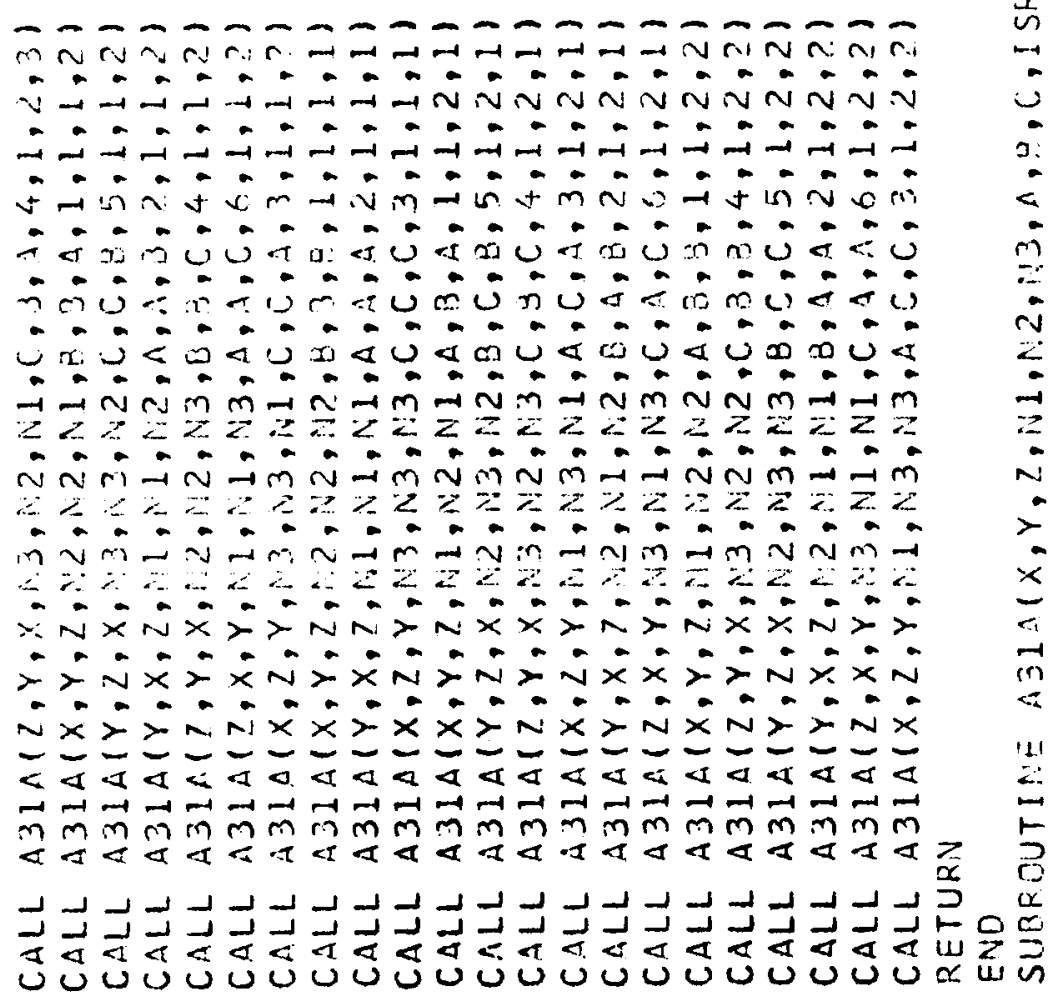



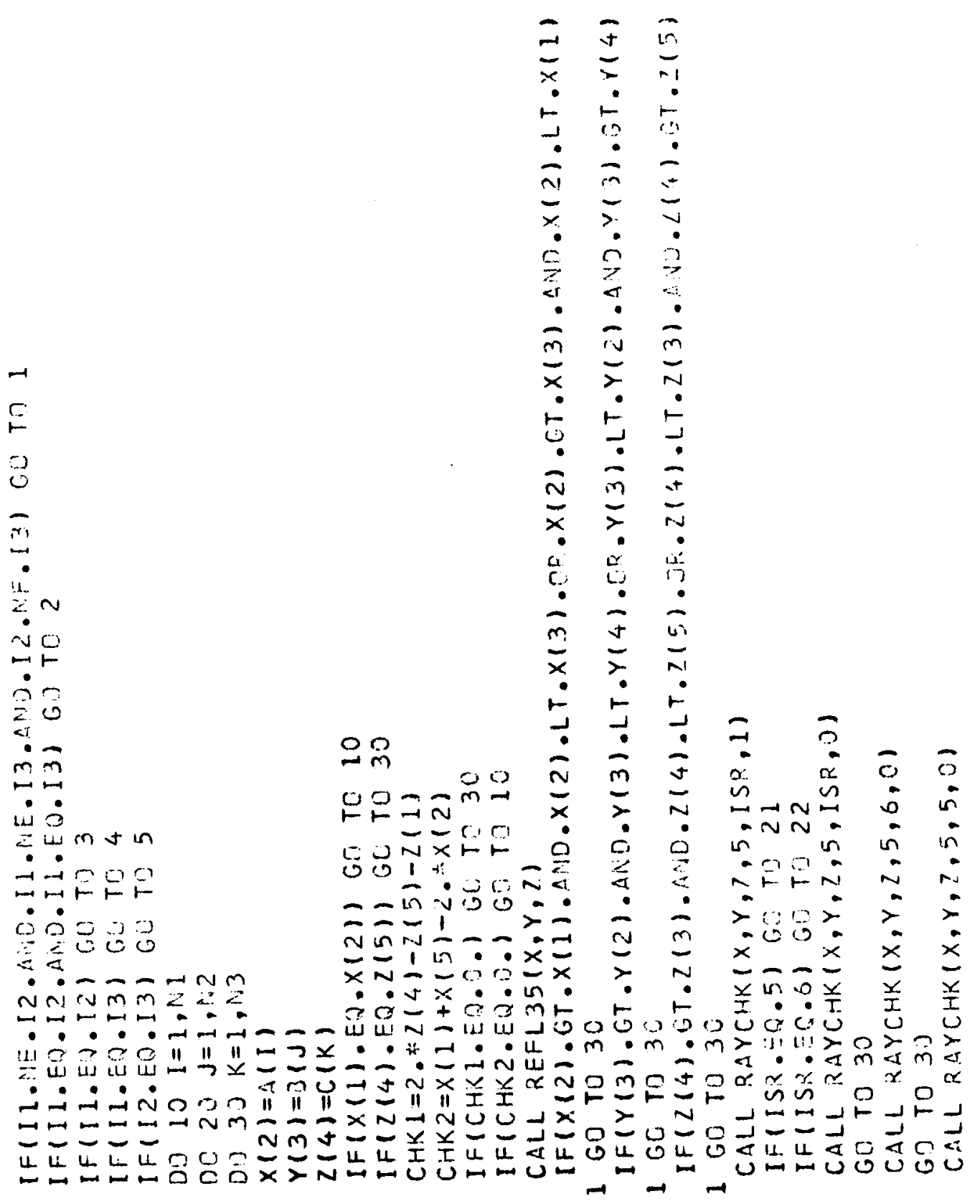

$-\rightarrow-m m$

Nol $v m \geq z$

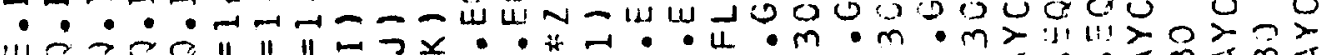
"1.! :. U.

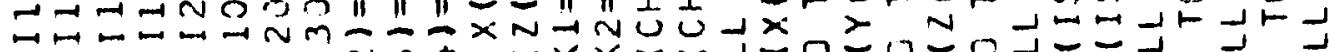
- б-

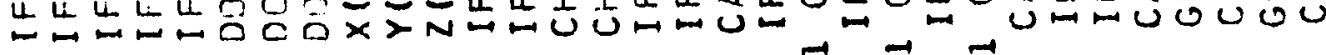




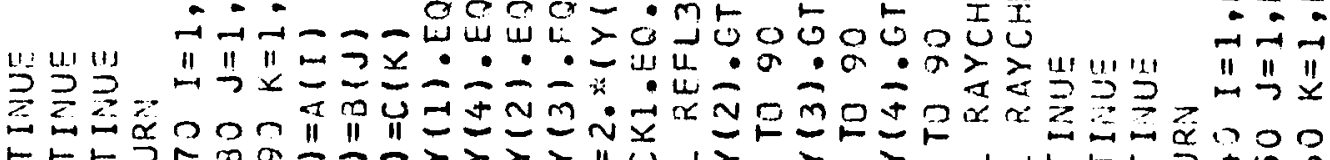

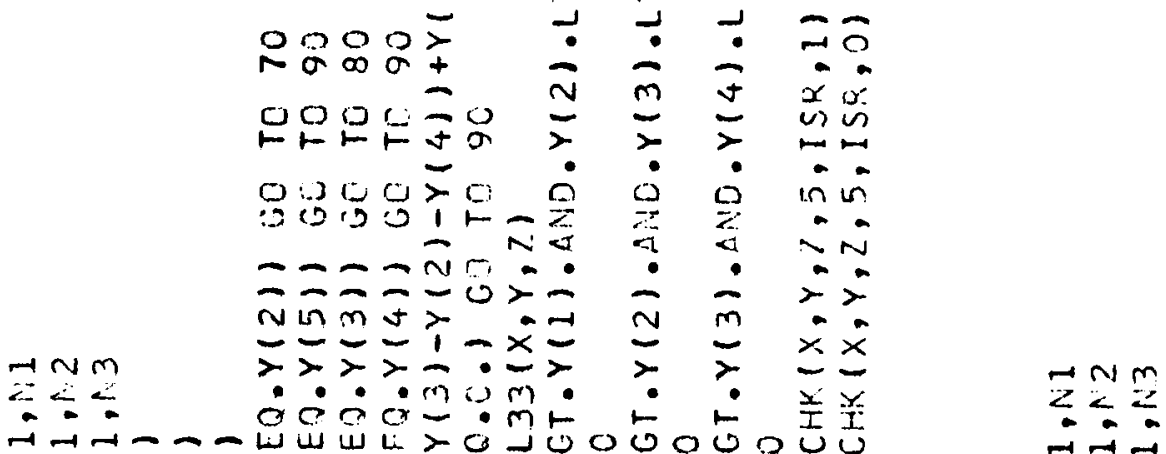

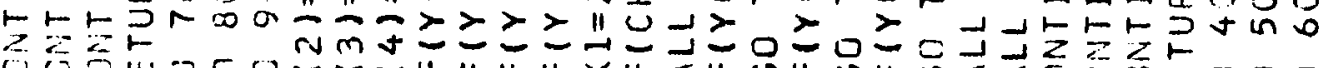

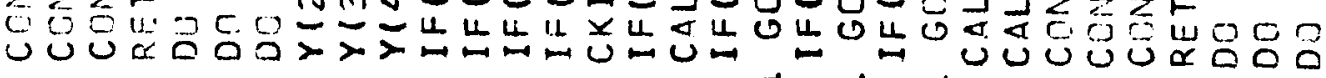
워은 


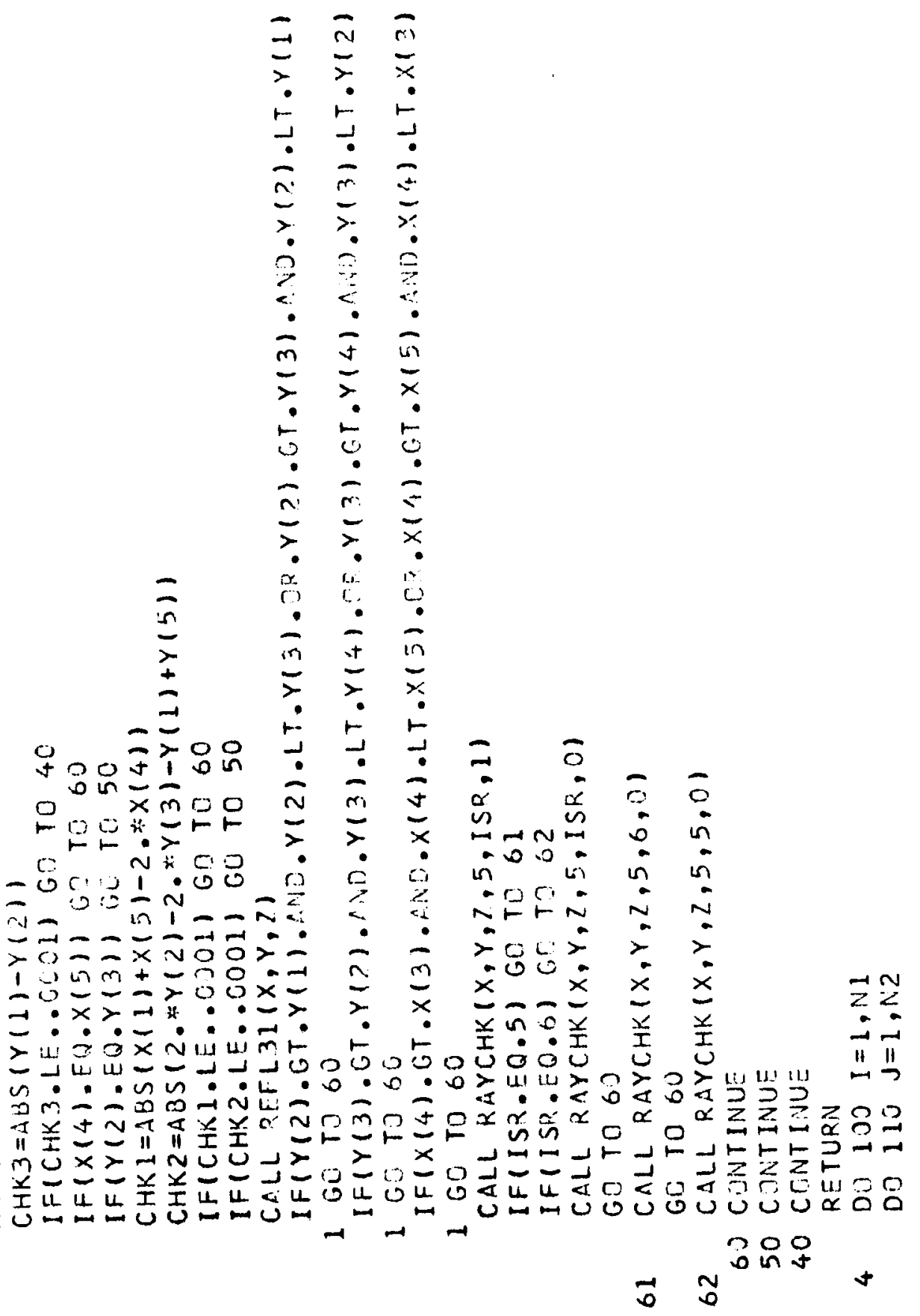




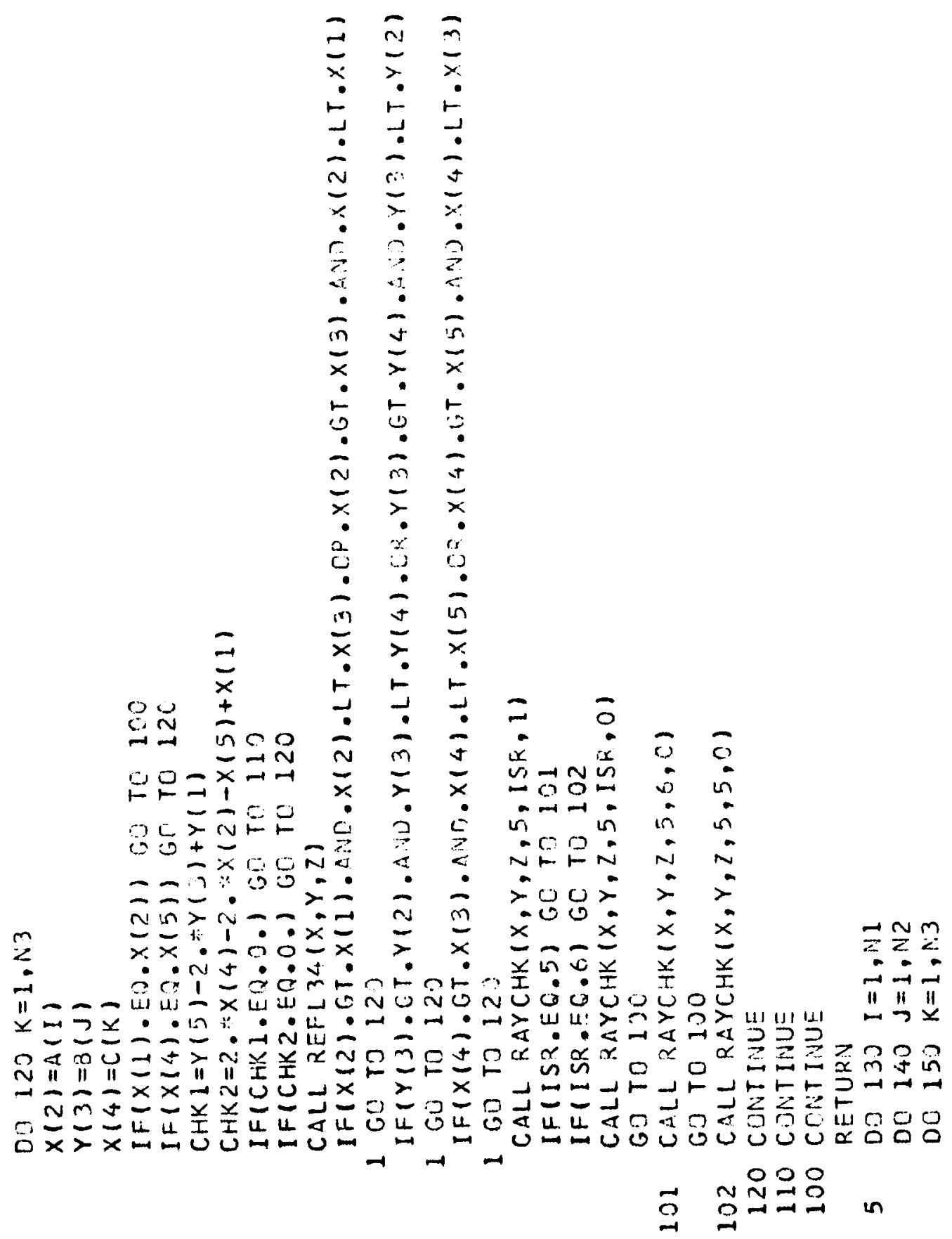




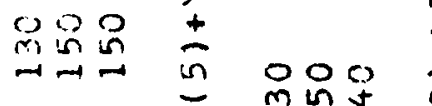

$\omega \boxminus E$ in

$\rightarrow \rightarrow-1$

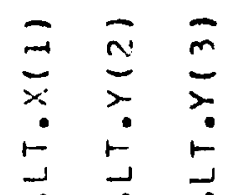

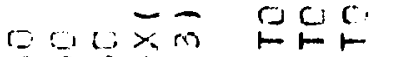

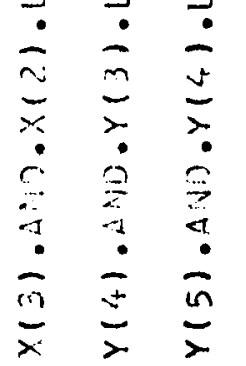

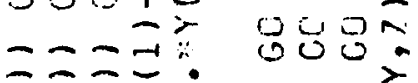

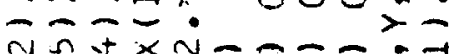

$N \Omega=x N=\div \div=$

$x>2-1=000-x$

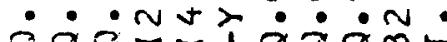

- - w

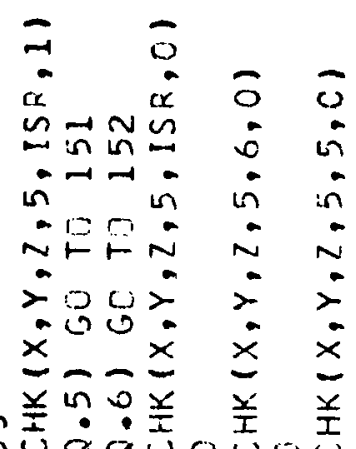

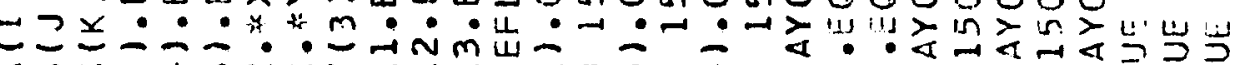
페

N⿳士コ一兀

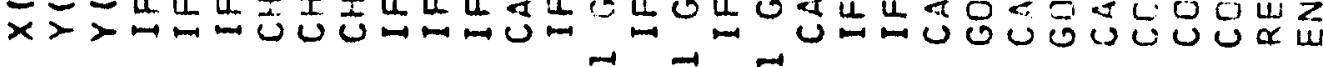




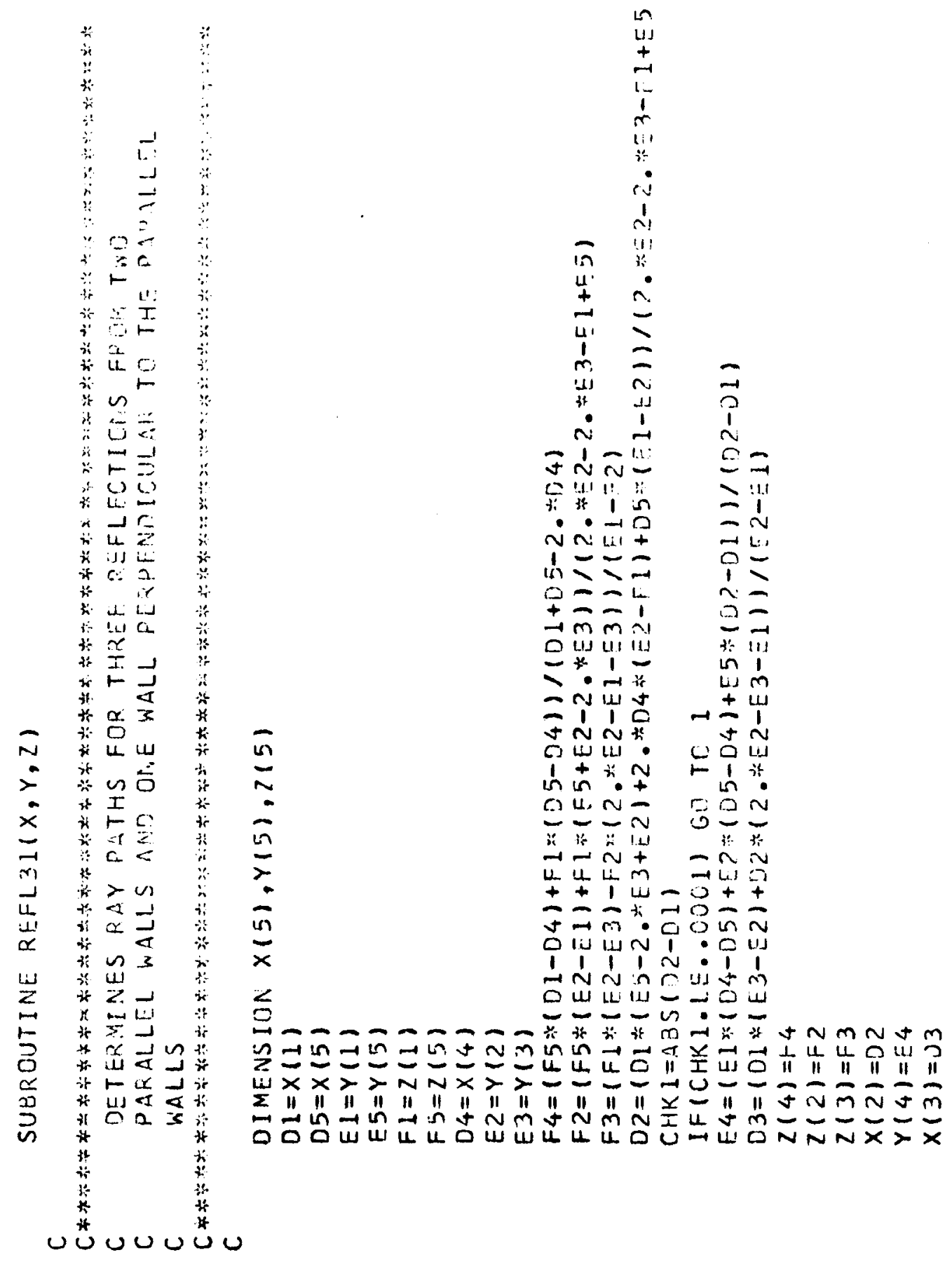




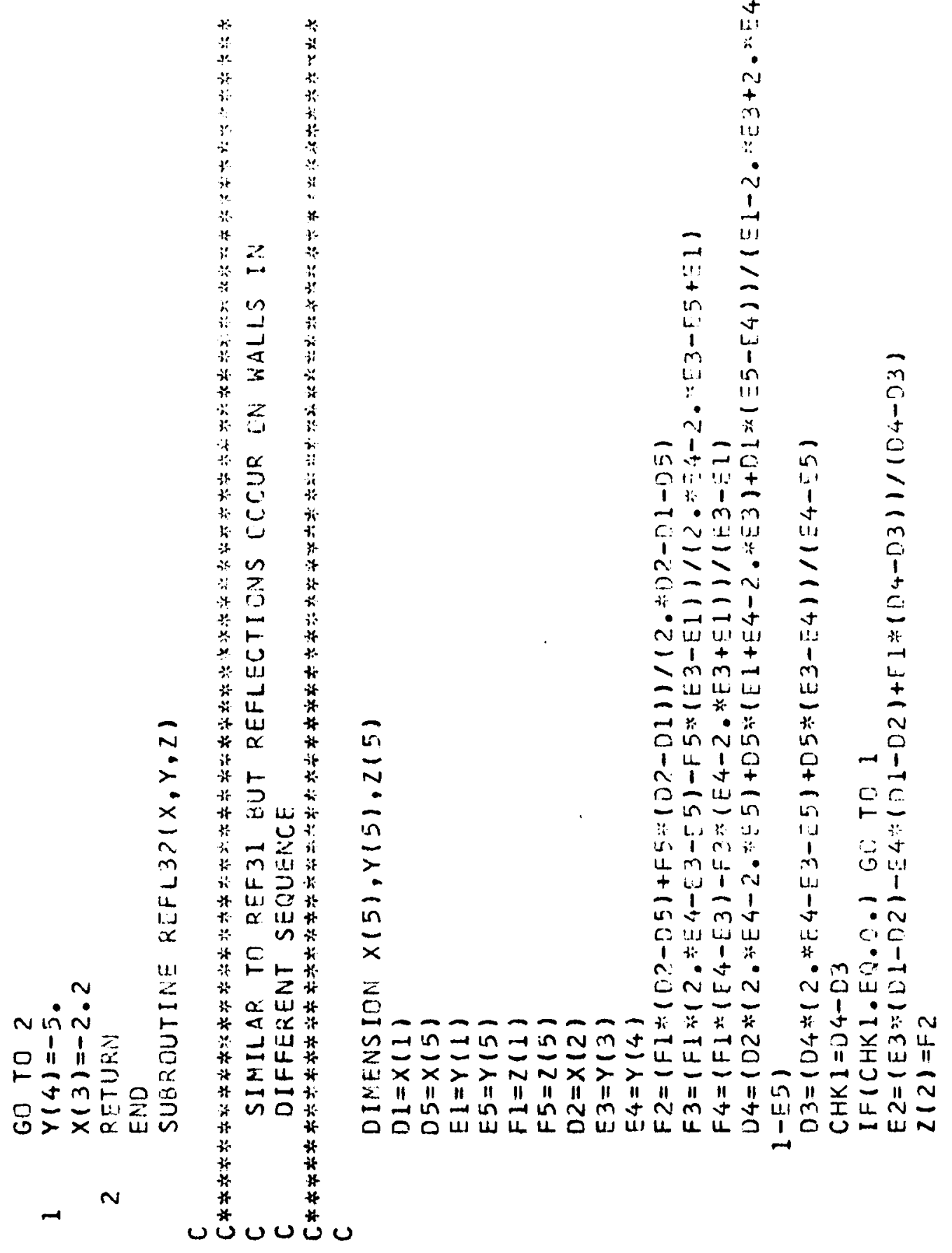




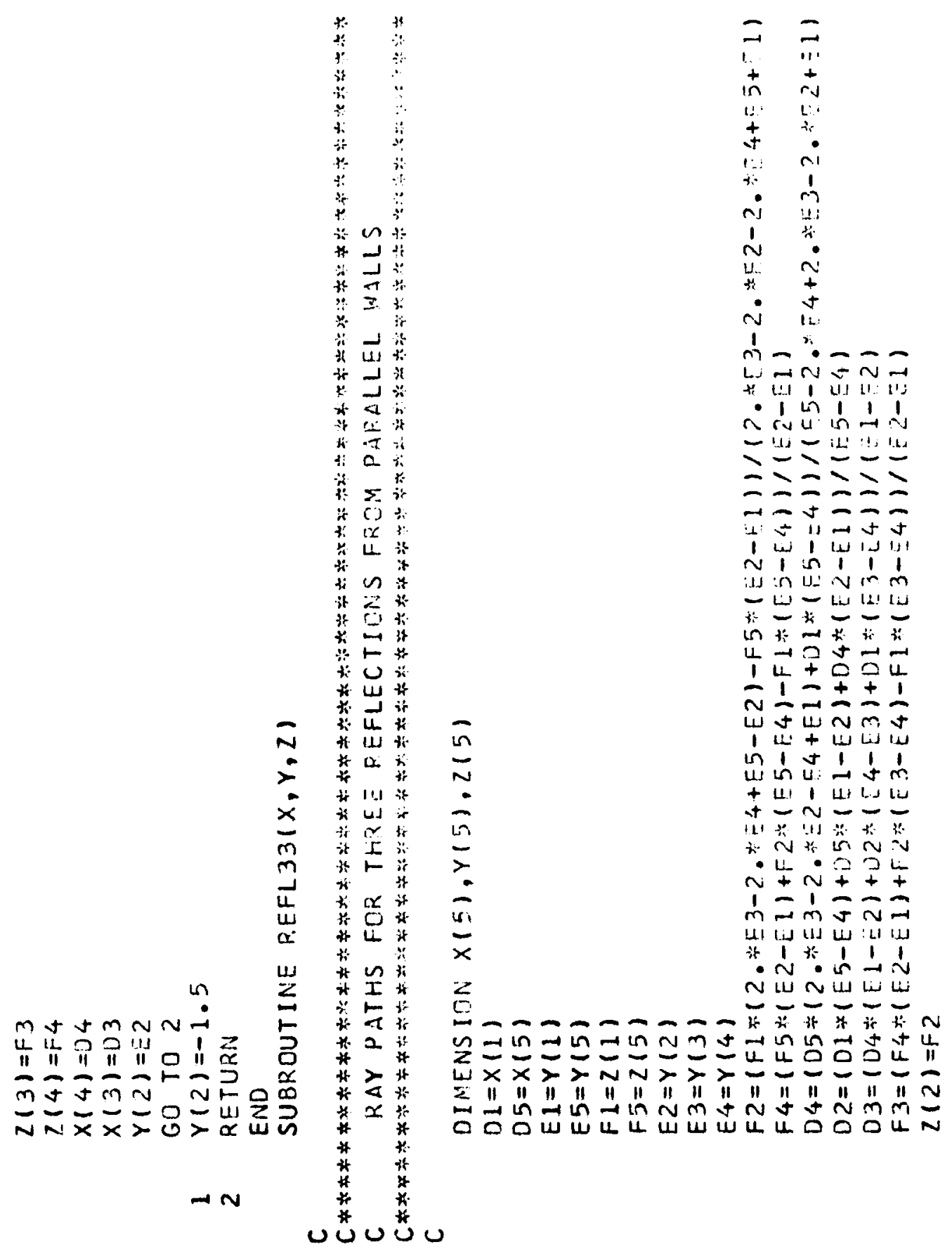




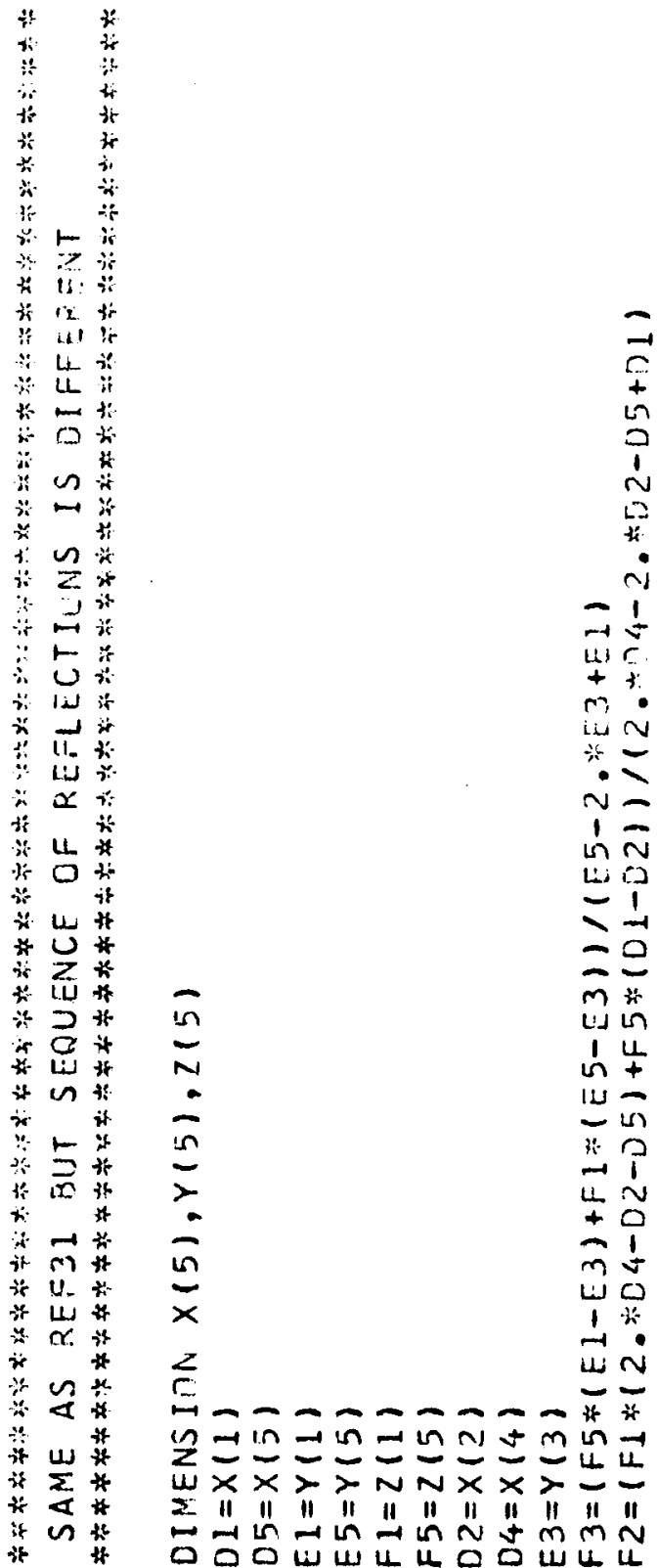




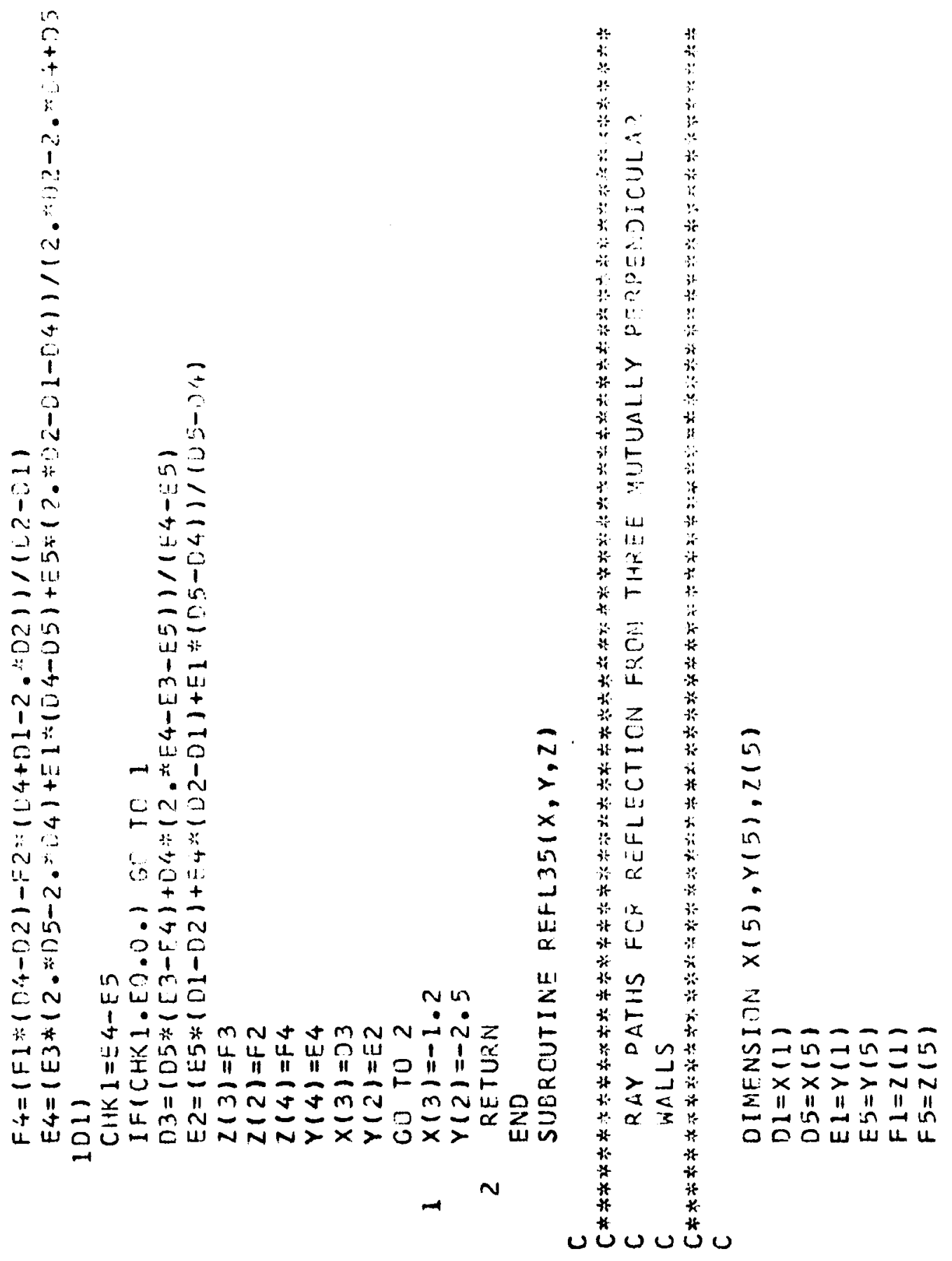




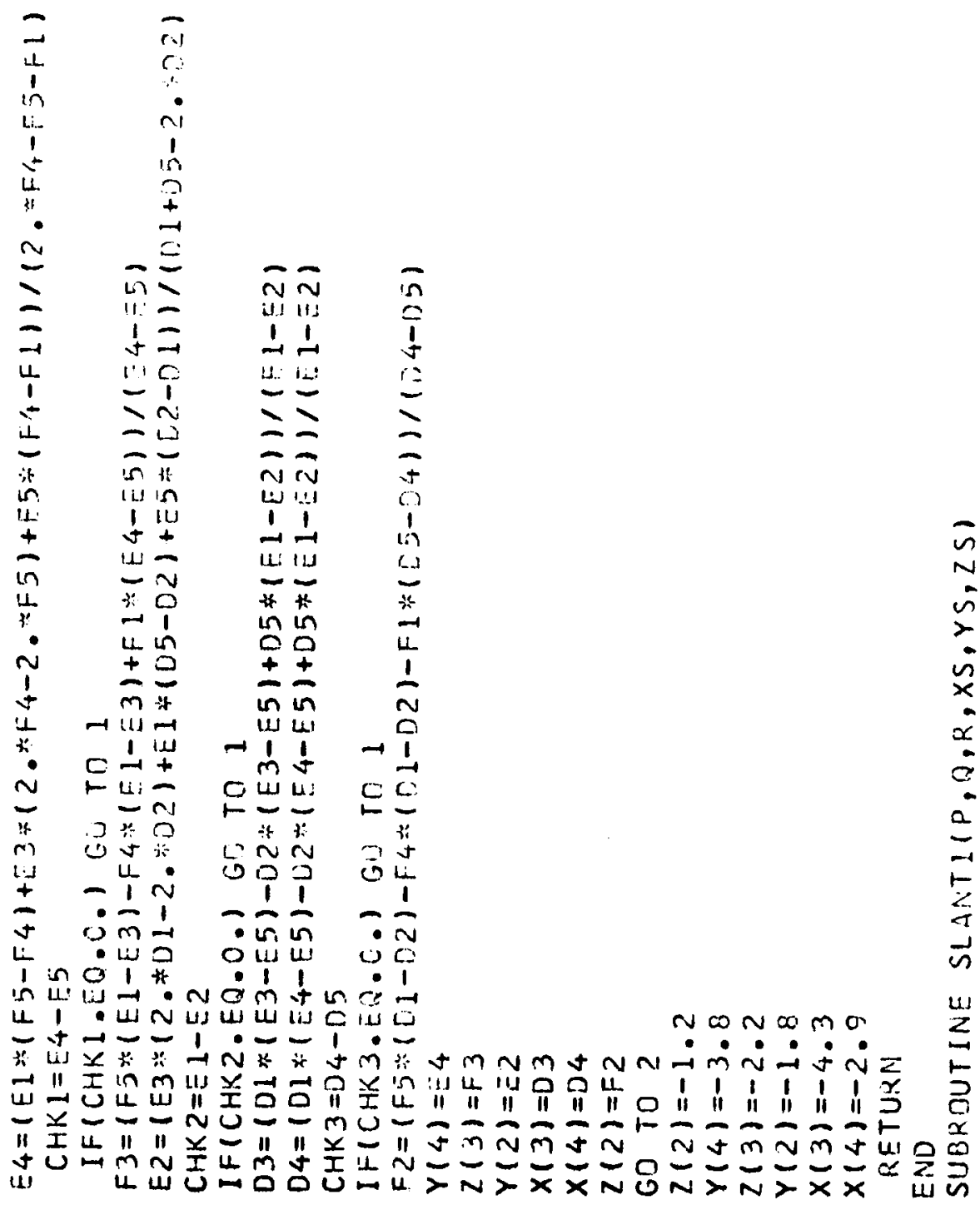

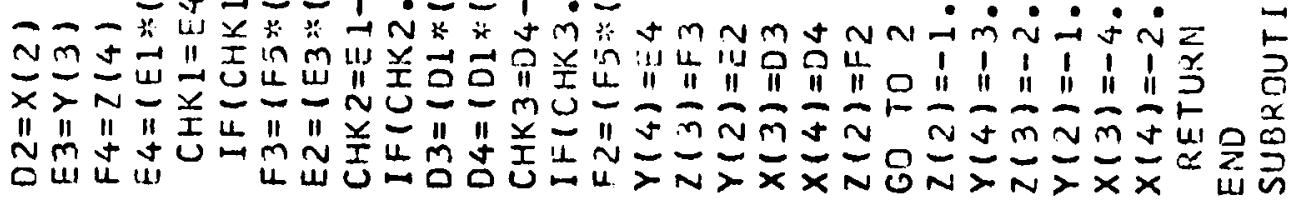



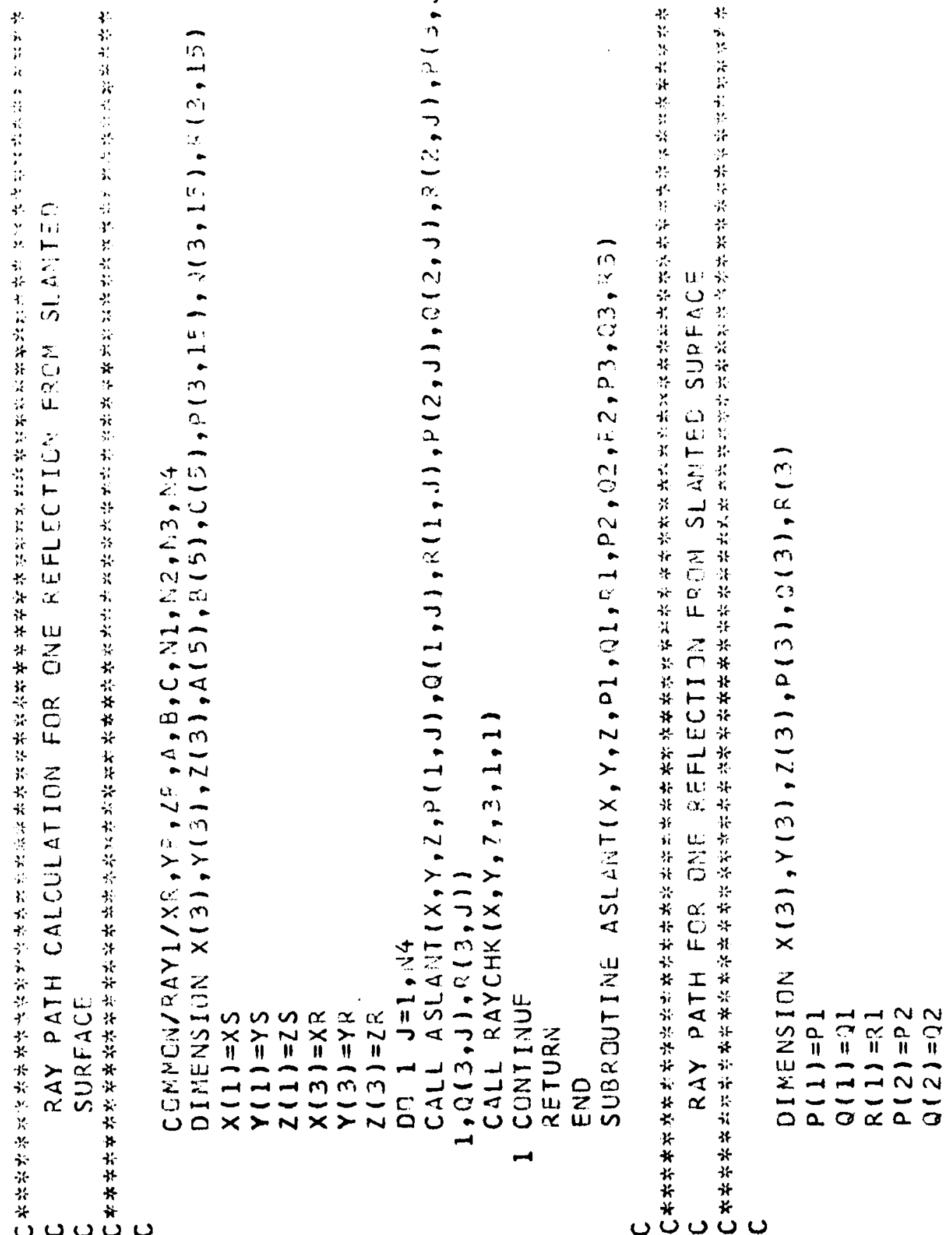
$\omega \sim \infty$

$P=$

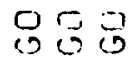
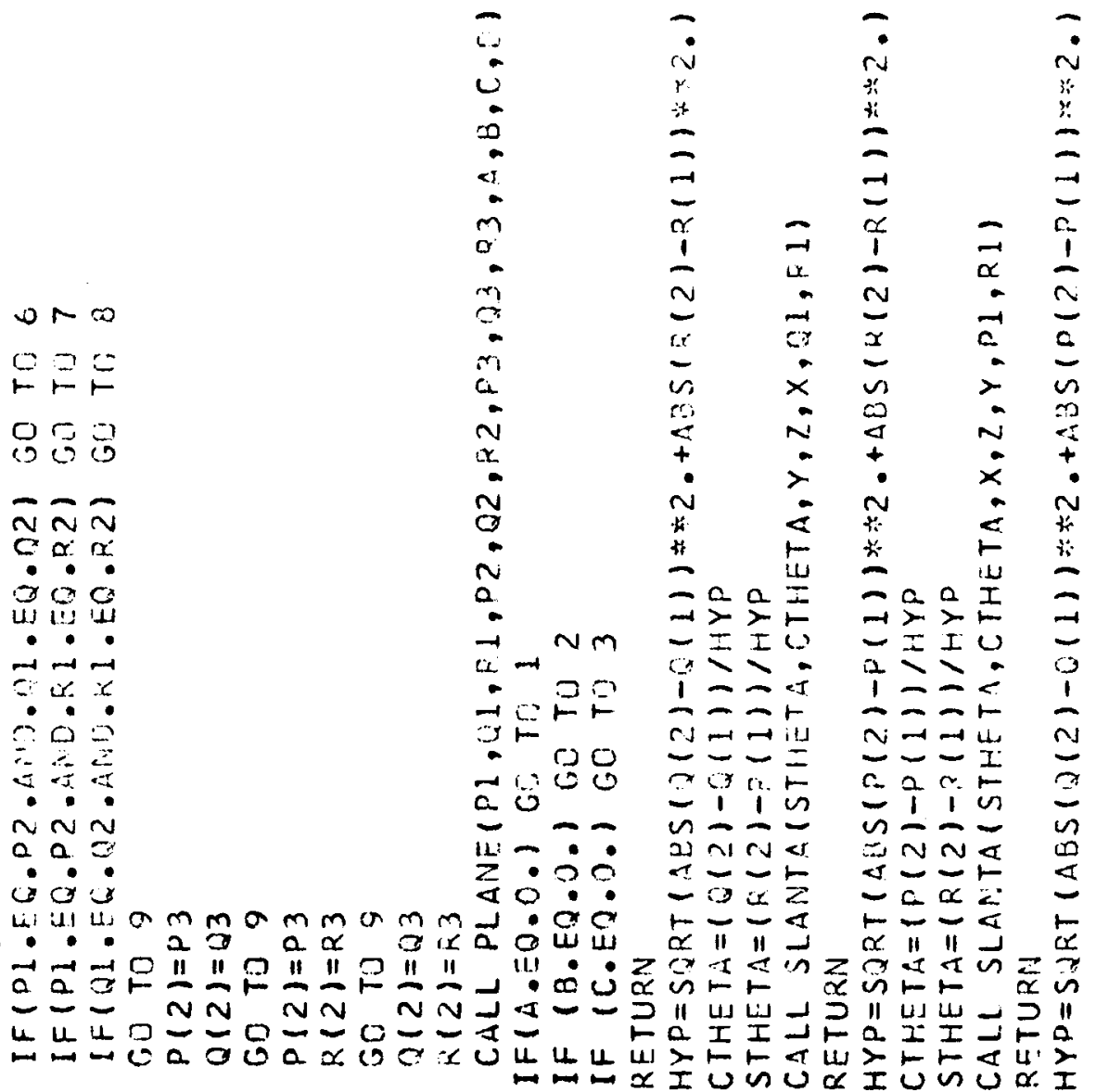

Nmmma a a a a a

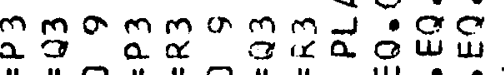

$\alpha$ "I 11

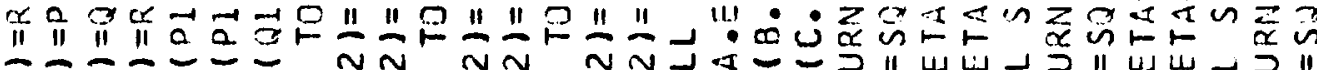

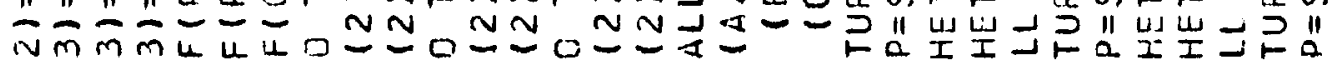

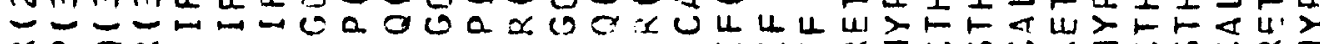
$10 \%$ $m m x I \omega \backsim \cup \propto I$

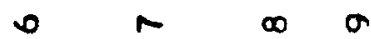




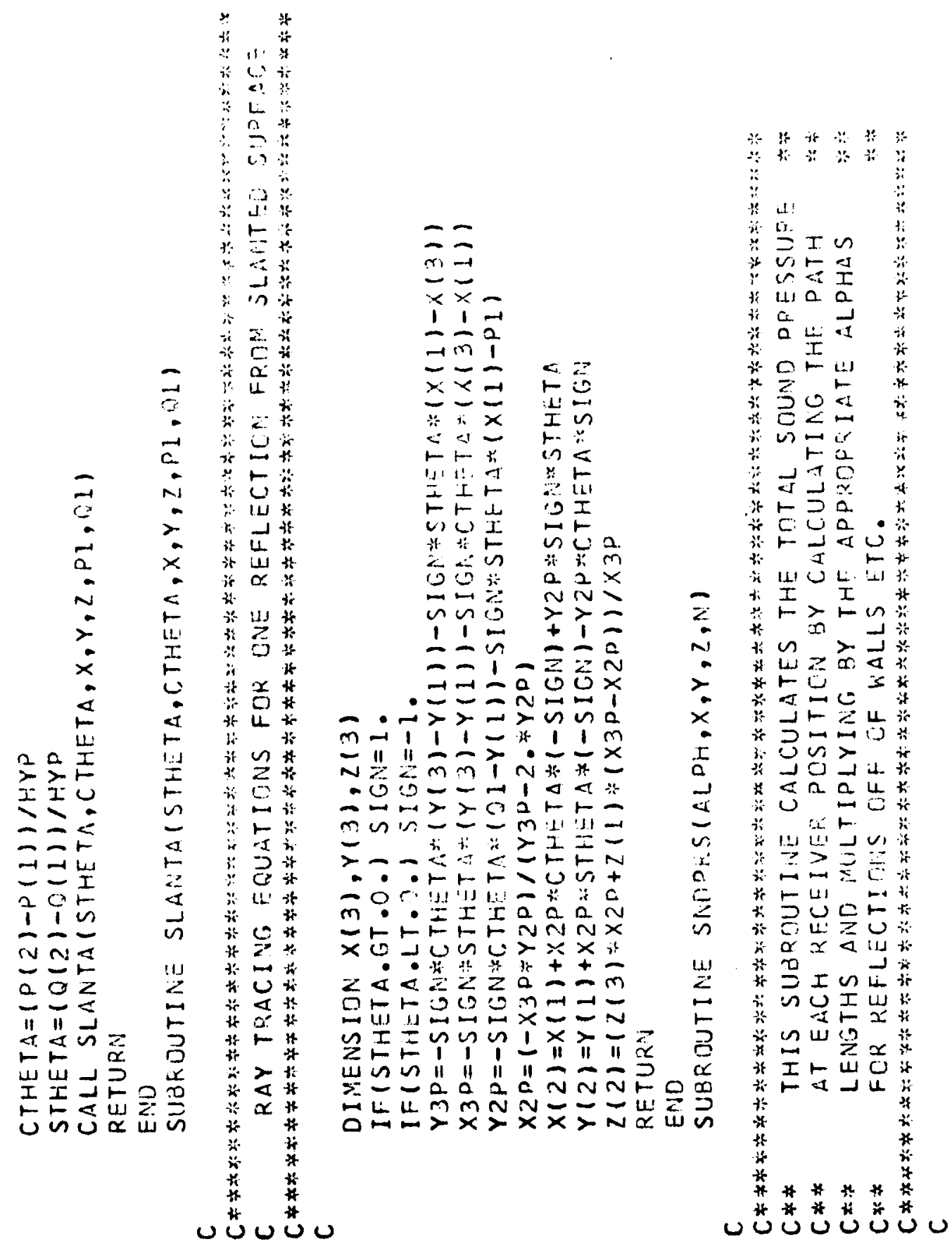




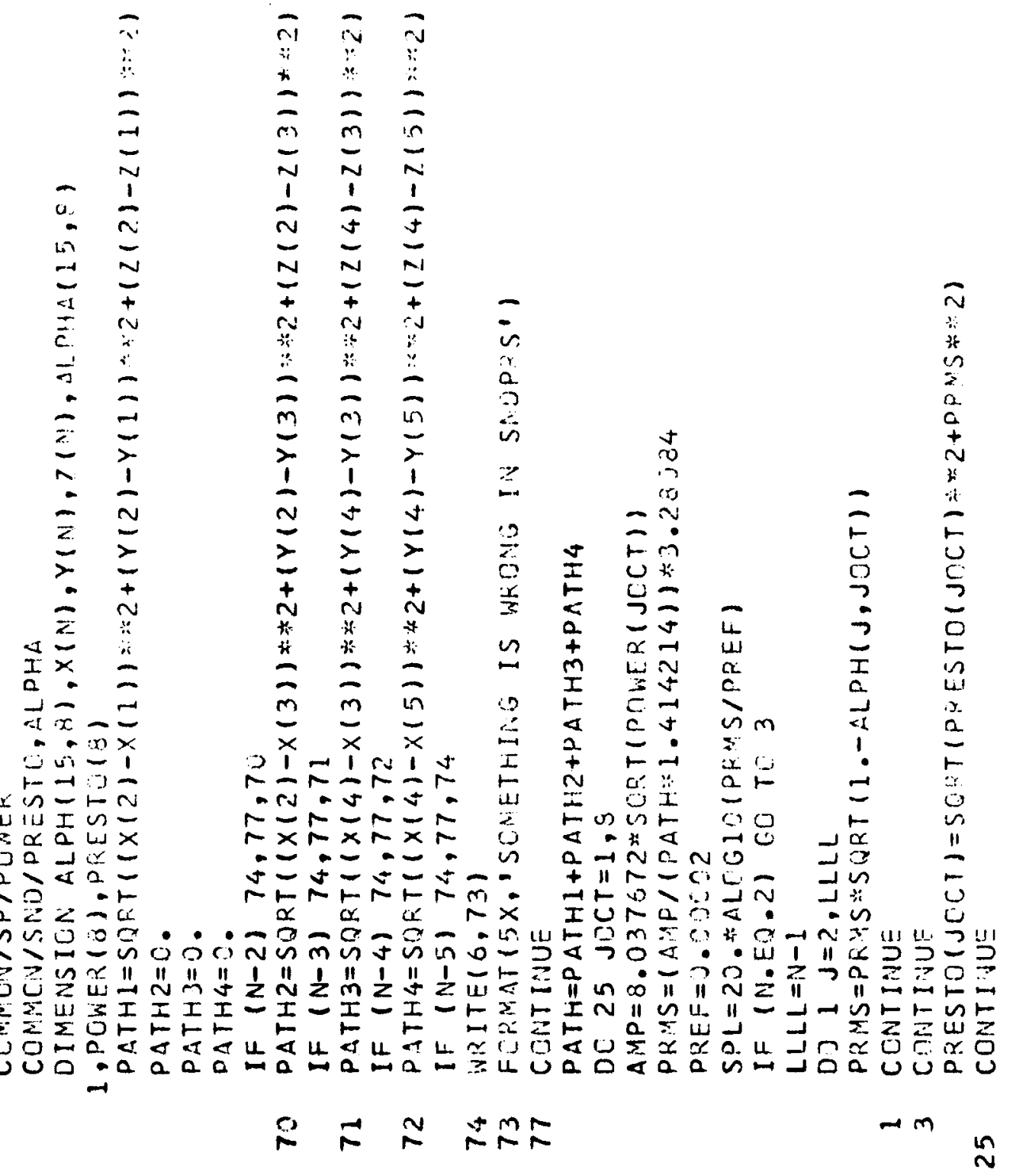



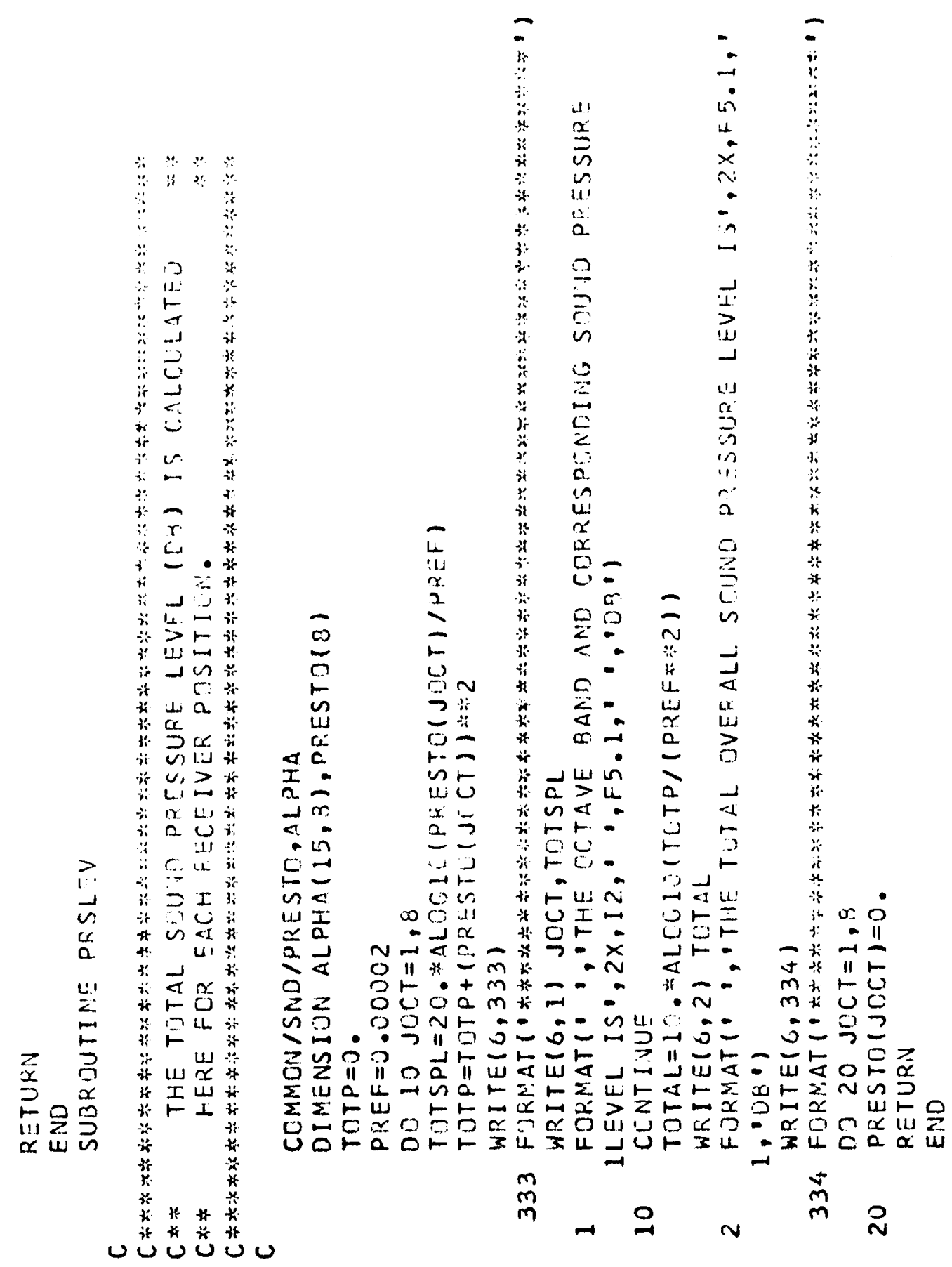


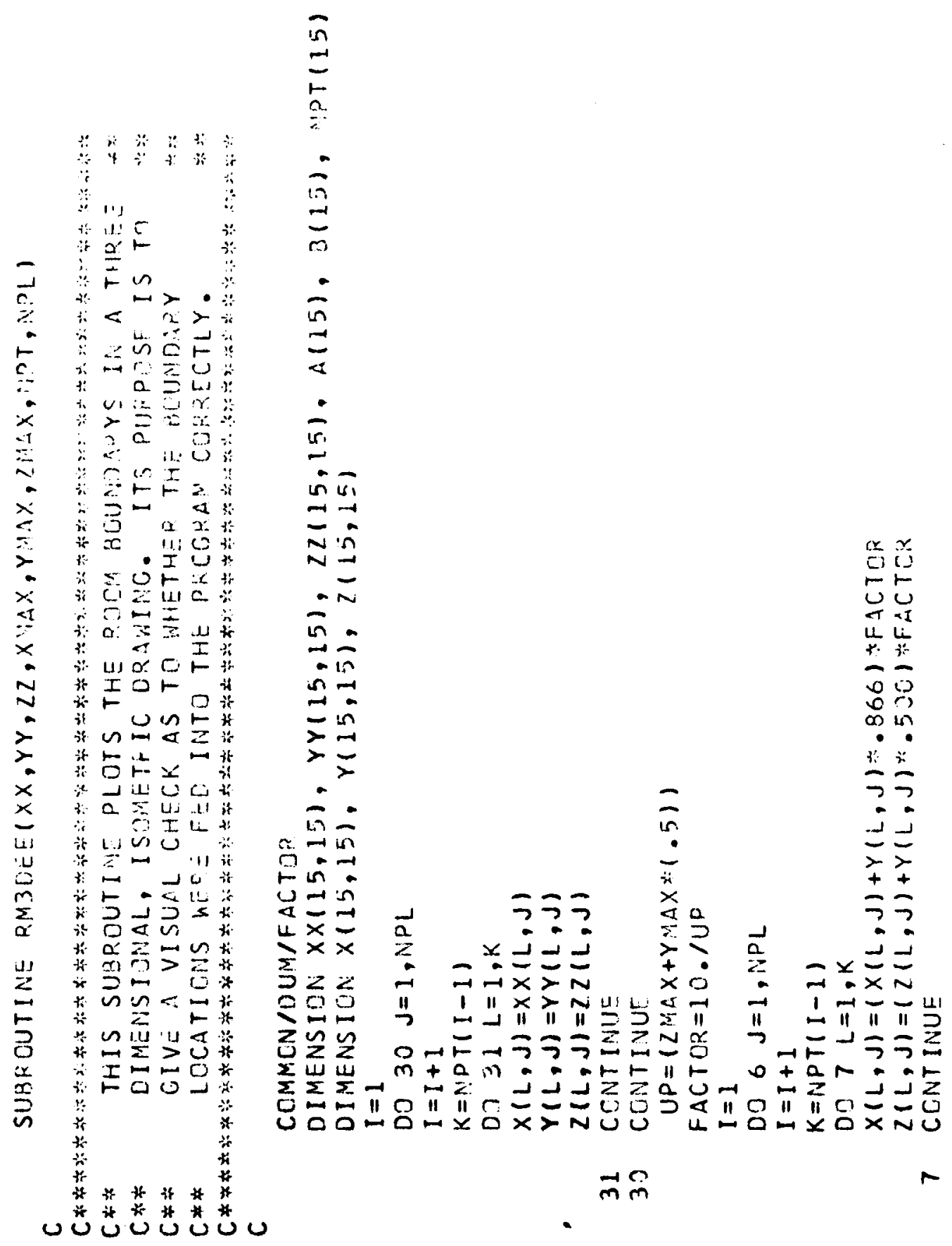



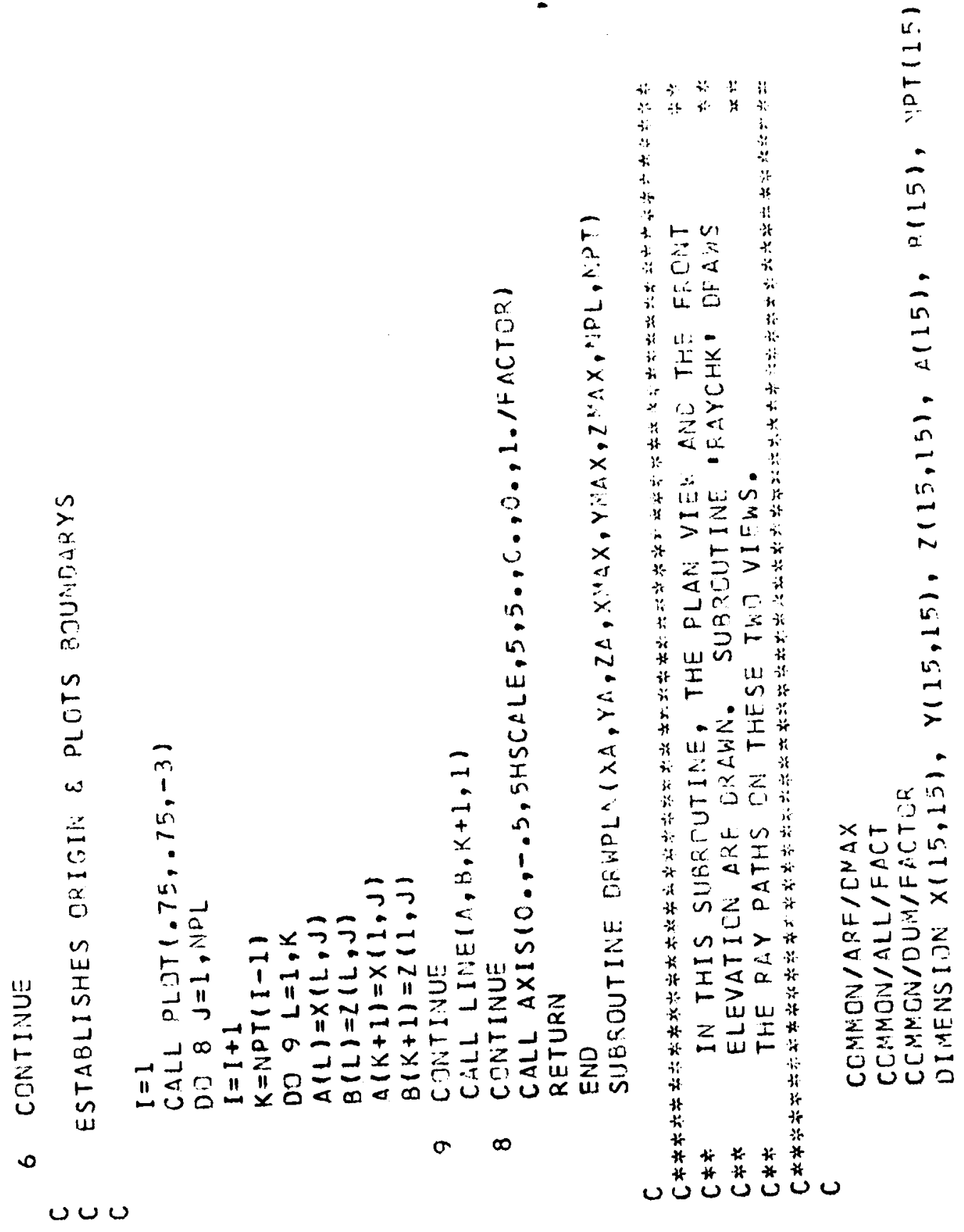


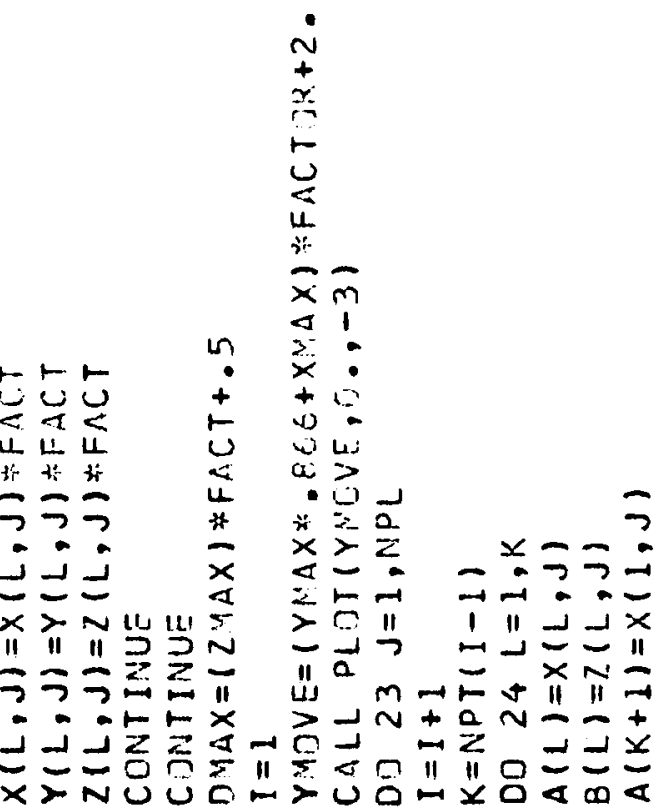

$\overrightarrow{m o}$

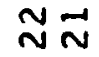




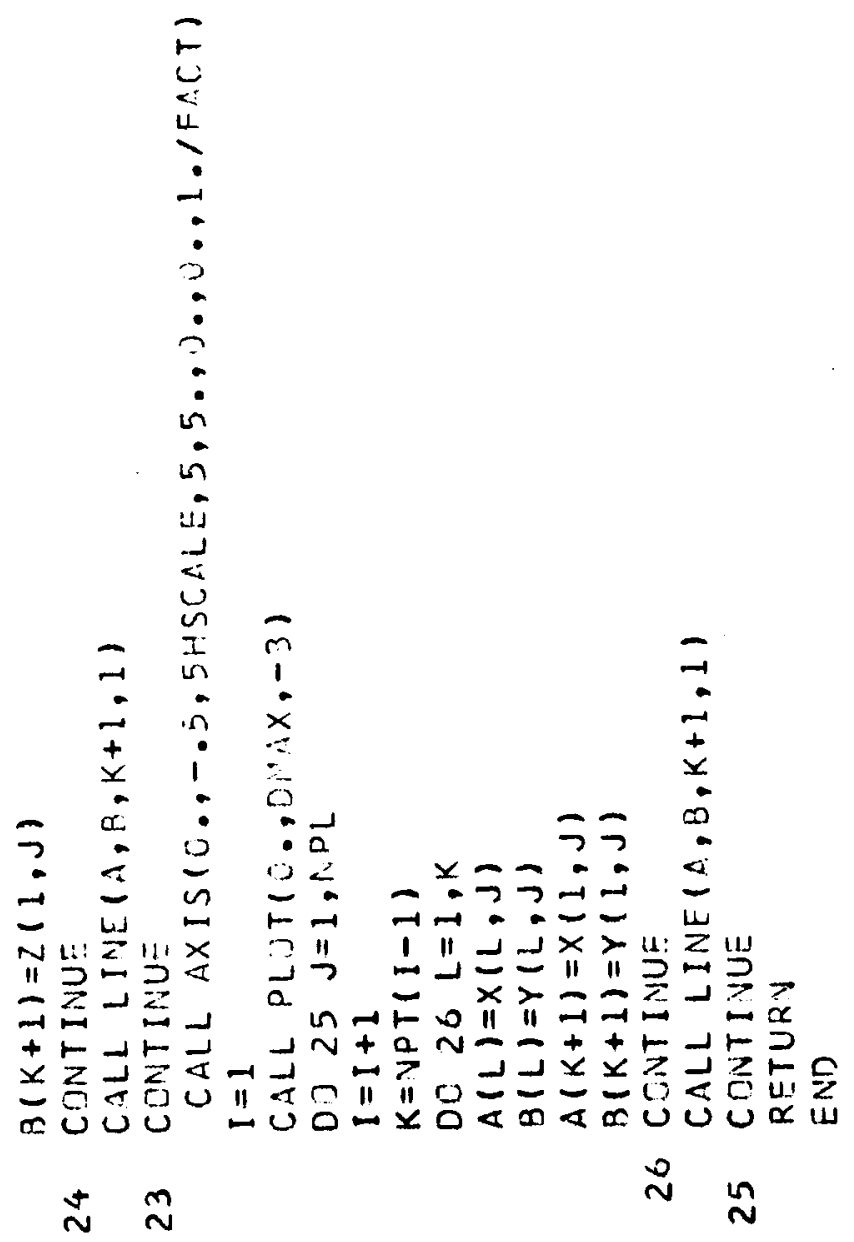




\section{The vita has been removed from the scanned document}




\title{
DETERMINATION OF ACOUSTIC RAY
}

PATHS IN ENCLOSED SPACES

by

\author{
Bruce Wayne Mitchell
}

(Abstract)

Equations were derived to calculate the reflection points of acoustic rays emanating from a source to a receiver. Reflection points for up to three reflections could be determined if the location of the source, receiver and all reflecting surfaces were known. Assuming a point source, the distances along the calculated ray paths were used along with the power level of the source and the absorptive characteristics of all the reflecting surfaces to determine the sound pressure levels at specified receiver locations. A computer program was developed to perform the necessary calculations for reflection points and sound pressure levels. The output was in the form of ray tracing plots which showed the unique reflection paths for up to three reflections and the sound pressure levels at each receiver location. Comparisons were made between predicted and calculated sound pressure levels in a rectangular parallelopiped shaped room and a long narrow hallway.

The results of this investigation showed a promising potential in the area of sound pressure level predictions and the use of ray tracing plots to provide a means of reducing the sound pressure levels. A particularly interesting point of the program was its ability to handle very irregularly shaped rooms which include slanted surfaces. 


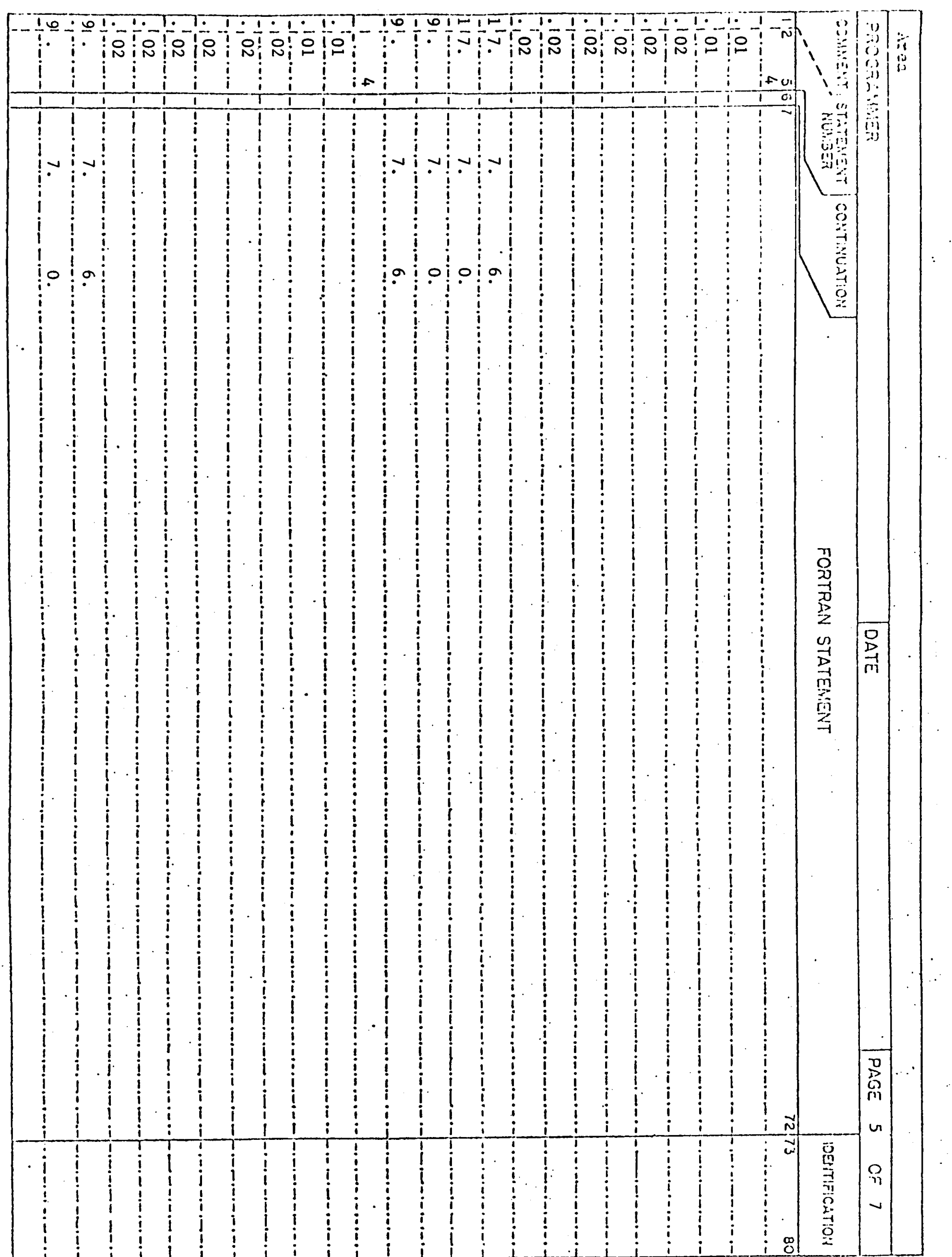




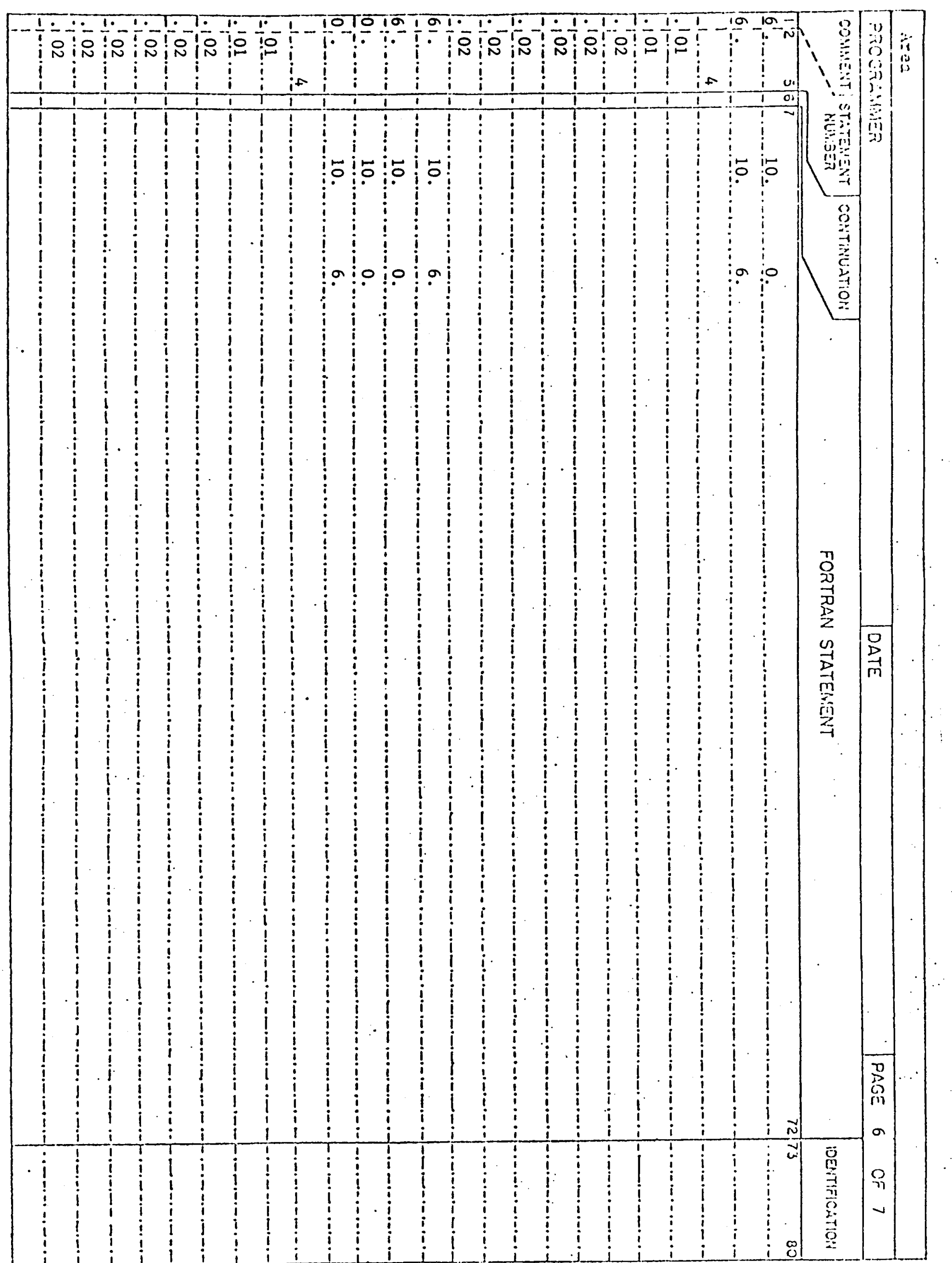






